\title{
Older Peoples' Attitudes to Mental IIIness
}

\author{
Kathryn M Quinn
}

August 2007

Thesis submitted to the University of Edinburgh in part fulfilment of the degree of Doctorate in Clinical Psychology 


\section{DECLARATION}

I declare that I am the sole author of this thesis and that the work contained herein is my own. This thesis, or any part of it, has not been submitted for any other degree or professional qualification.

Signed:

Kathryn M Quinn

Date: 191.10107 


\section{TABLE OF CONTENTS}

LIST OF TABLES ................................................................................ vi

LIST OF APPENDICES ........................................................................... vii

ABSTRACT ................................................................................................... ix

1. INTRODUCTION ........................................................................................................... 1

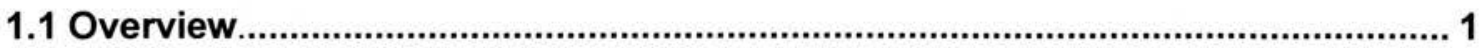

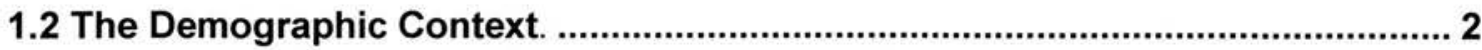

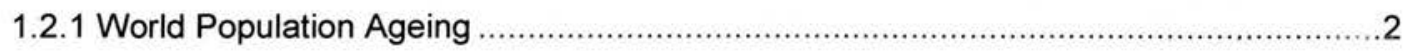

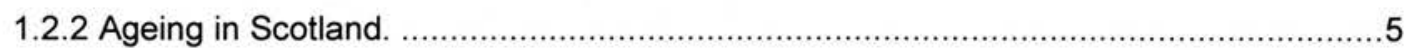

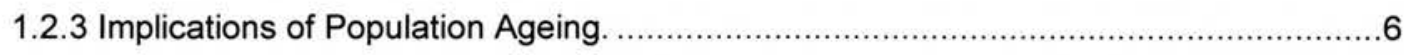

1.3 A Scottish Policy Perspective.......................................................................... 7

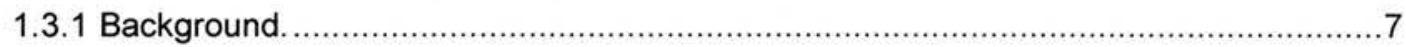

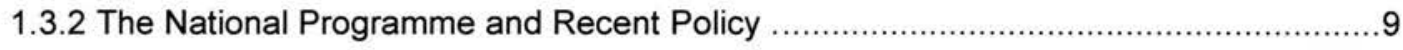

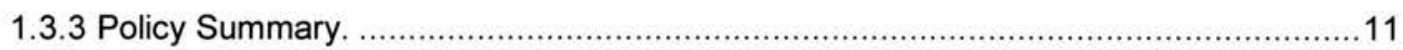

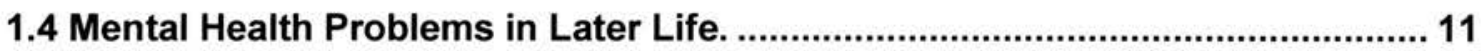

1.4.1 Factors Affecting Recognition, Diagnosis and Treatment.....................................11

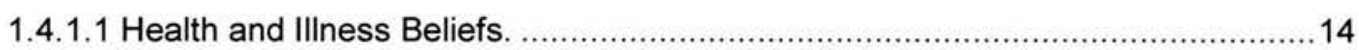

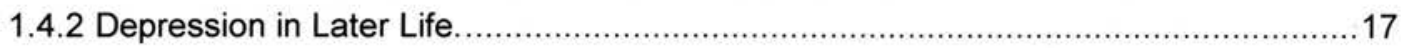

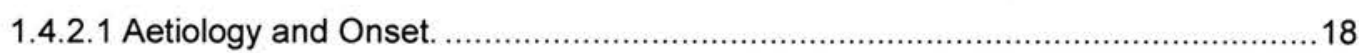

1.4.2.2 Prevalence of Depression in Later Life. ......................................................... 19

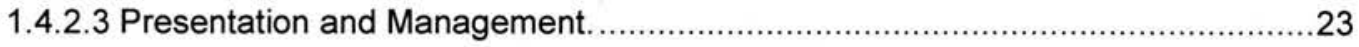

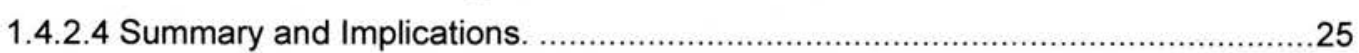

1.4.3 Implications for Health Service Utilisation and Current Research. ...........................26

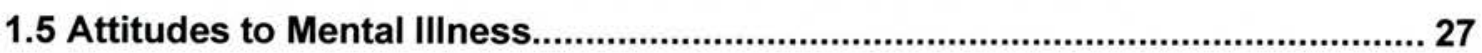

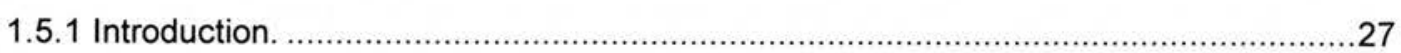

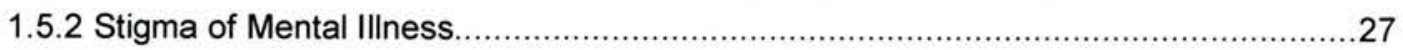

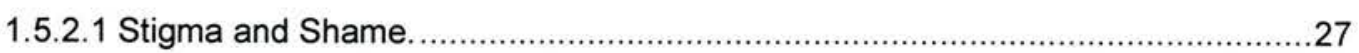

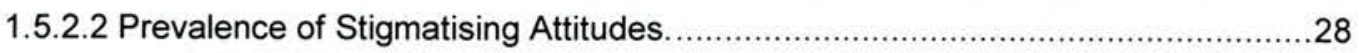

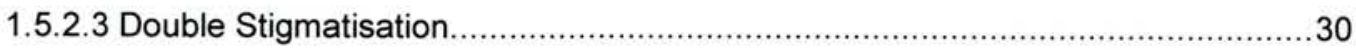


1.5.3 Cohort Effects and Inter-generational Differences ...........................................31

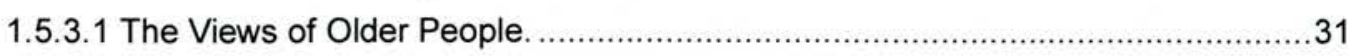

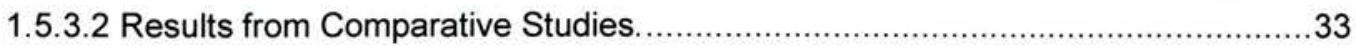

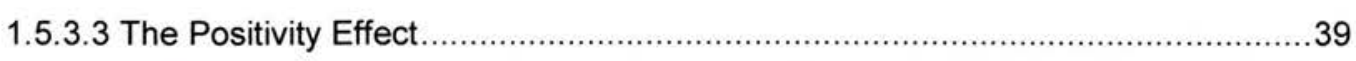

1.5.3.4 Summary of Attitudinal Research Relating to Mental IIIness............................40

1.6 Experience and Awareness of Mental Health Issues. .....................................41

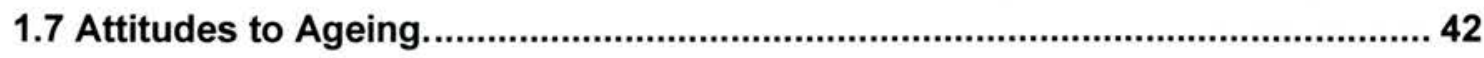

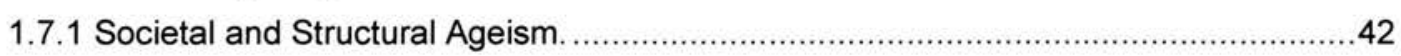

1.7.2 Negative Stereotypes of Ageing and Internalised Ageism. ...............................43

1.7.3 Positive Stereotypes and Successful Ageing. ..................................................46

1.8 Summary of Main Research Findings. .................................................... 48

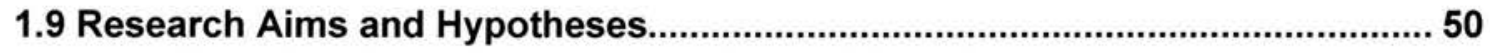

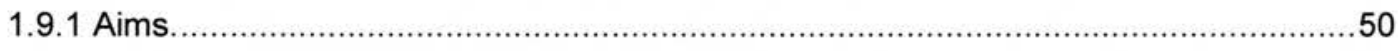

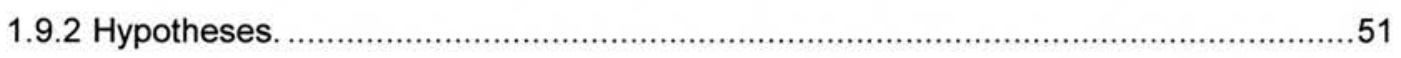

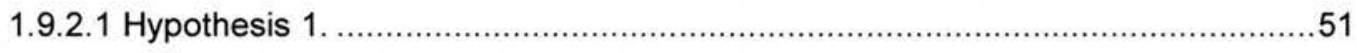

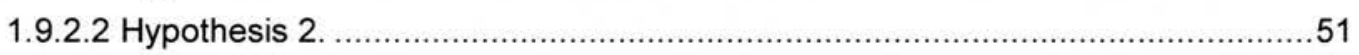

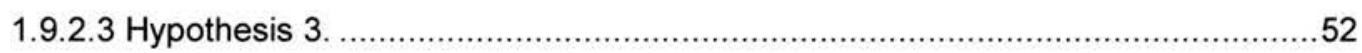

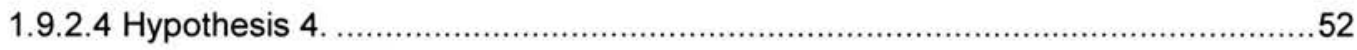

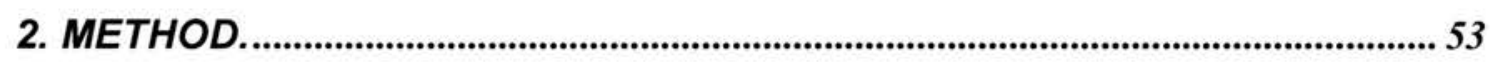

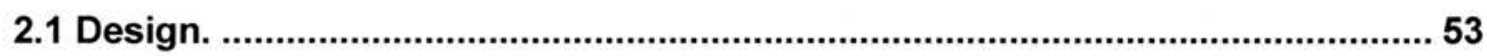

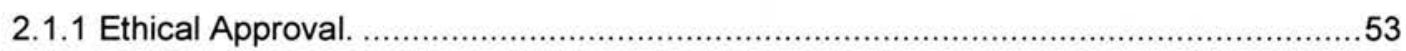

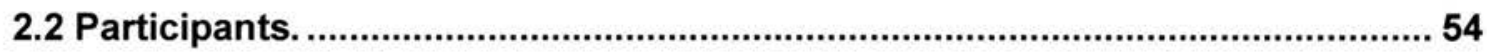

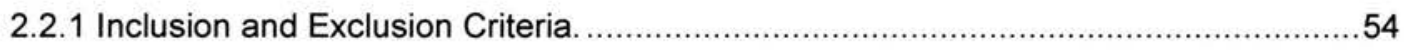

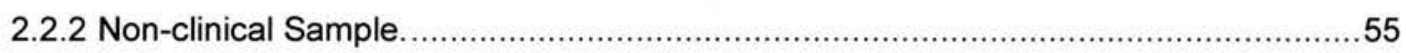

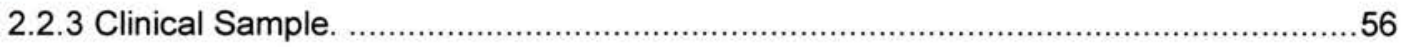

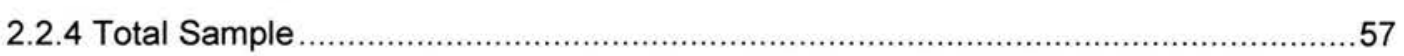

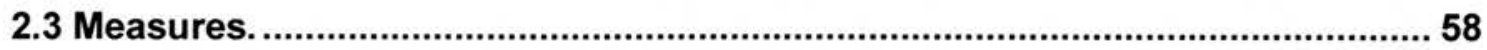

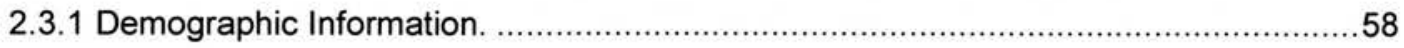

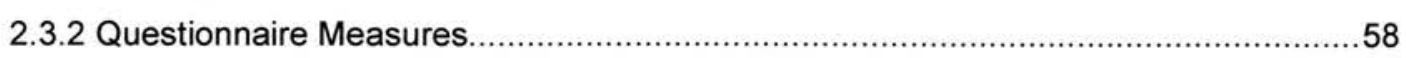

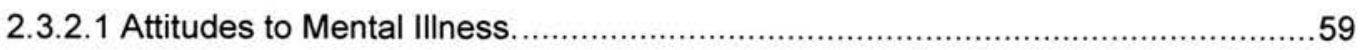

2.3.2.1.1 Items from the National Survey. ..................................................... 59

2.3.2.1.2 Barriers to Mental Health Services Scale ...............................................59 


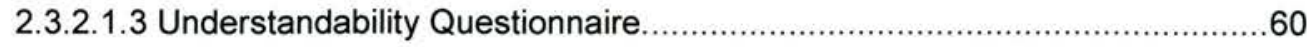

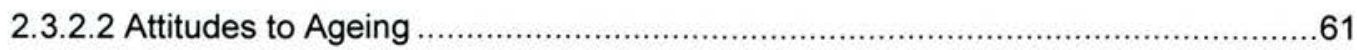

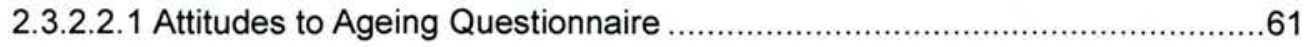

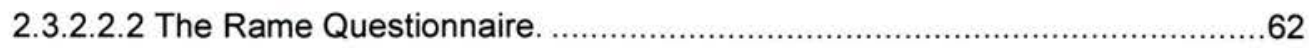

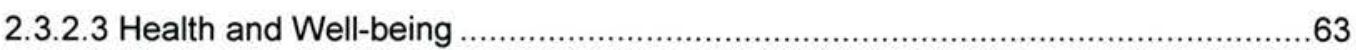

2.3.2.3.1 The General Health Questionnaire .......................................................63

2.3.2.3.2 Reported Health Behaviours Checklist ....................................................64

2.4 Focus Group Procedure.................................................................................. 65

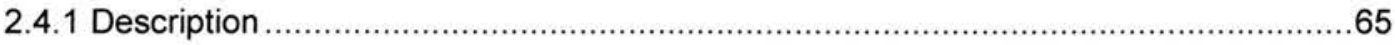

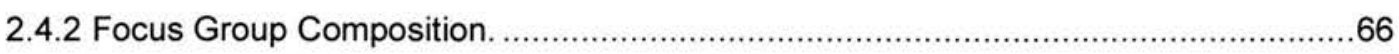

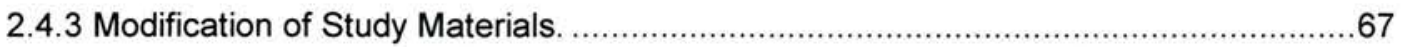

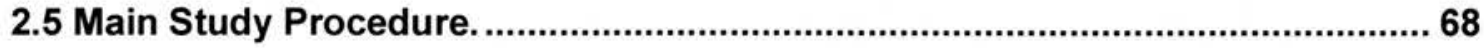

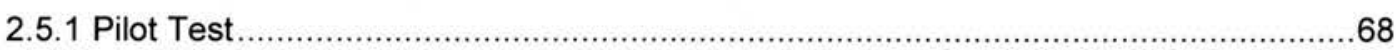

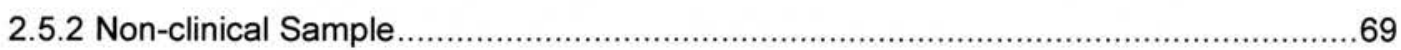

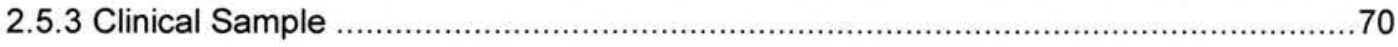

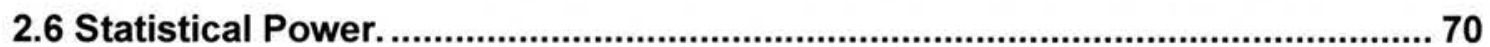

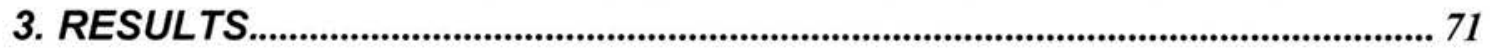

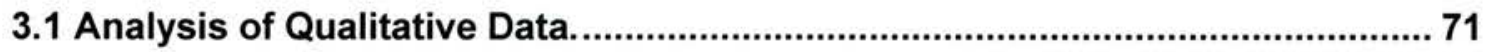

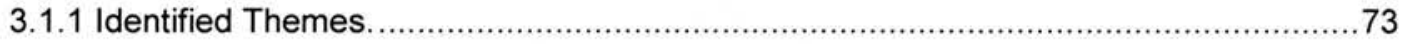

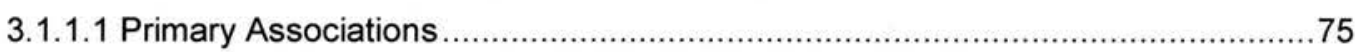

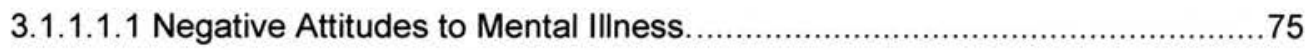

3.1.1.1.2 Positive Attitudes to Mental Illness. .....................................................

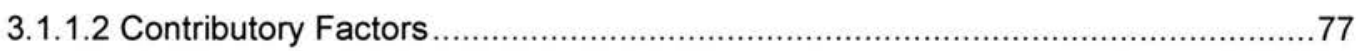

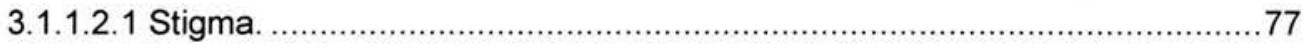

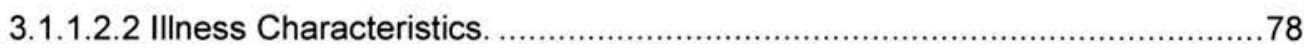

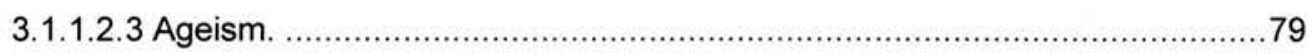

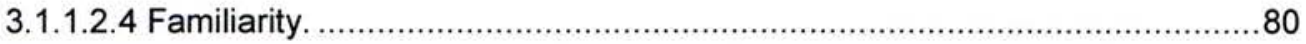

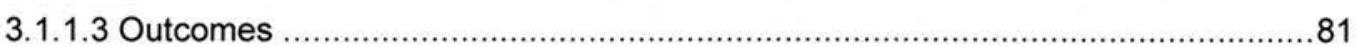

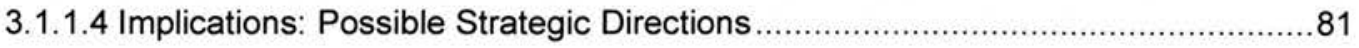

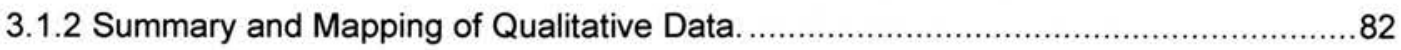

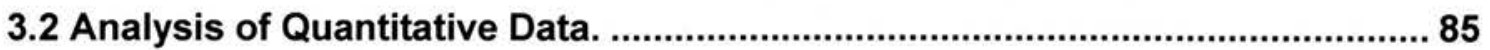

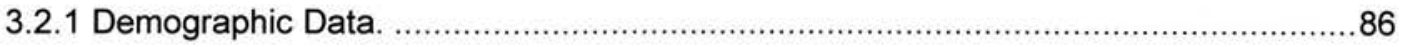

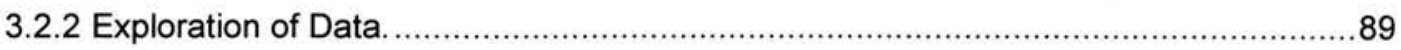




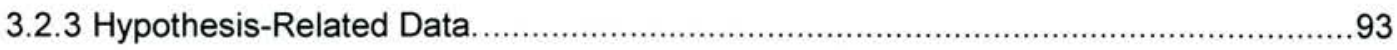

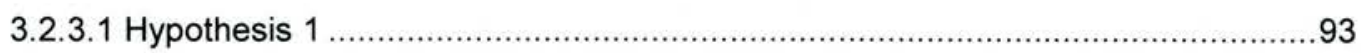

3.2.3.1.1 Between-group Analyses: National Survey Items. ................................93

3.2.3.1.2 Comparison of Attitudes to Mental IIIness in Younger and Older People. ...96

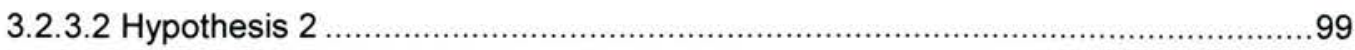

3.2.3.2.1 Between-group Analyses: Attitudes to Mental Illness in Later Life............99

3.2.3.2.2 Comparison of Attitudes in Those With and Without Prior Experience......101

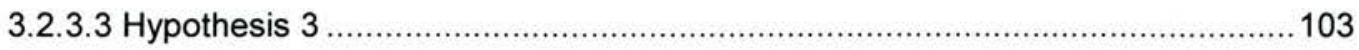

3.2.3.3.1 Between-group Analyses: Attitudes to Ageing. ................................... 103

3.2.3.3.2 Association between Attitudes to Ageing and Attitudes to Mental Illness.. 105

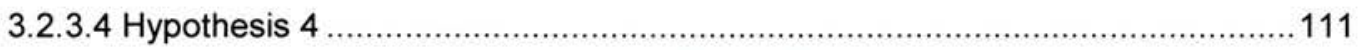

3.2.3.4.1 Between-group Analyses: RHBC and GHQ-12 ................................ 111

3.2.3.4.2 Association between Health Behaviours, Well-Being and Attitudes..........113

3.2.4 Summary of Results .................................................................................. 118

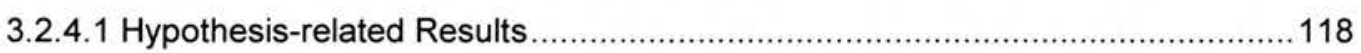

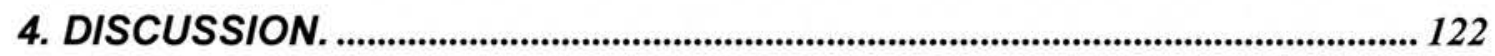

4.1 Overview and Summary of Study Findings..........................................................122

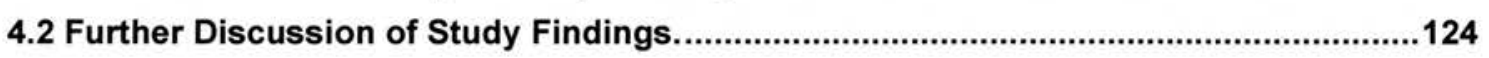

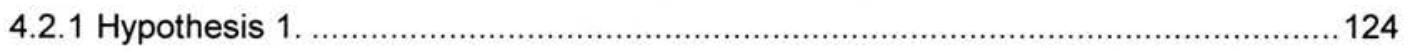

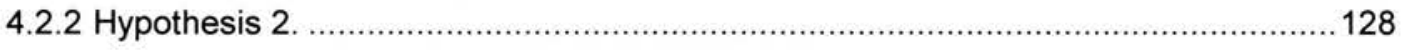

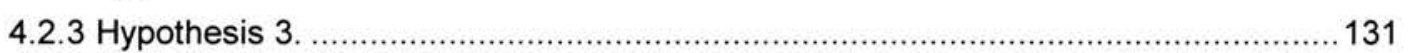

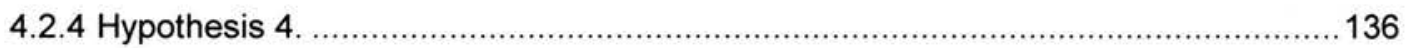

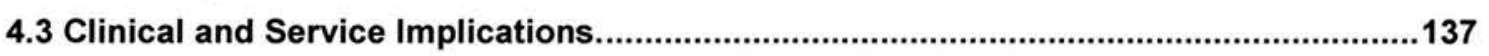

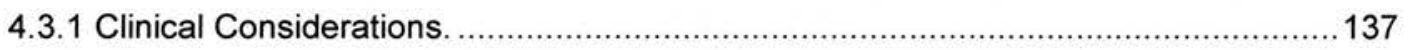

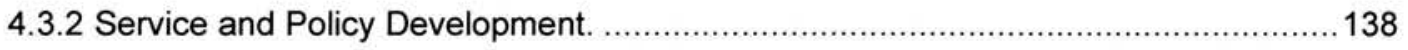

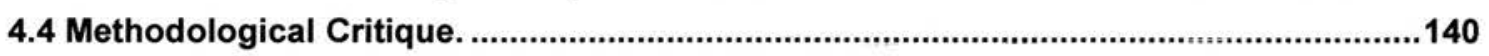

4.4.1 Mixed Methodology Design...................................................................... 140

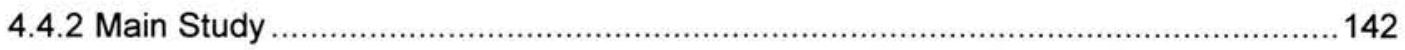

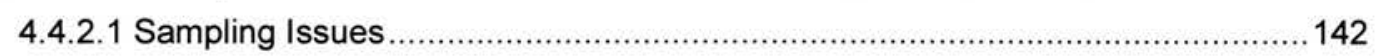

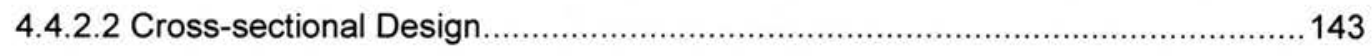

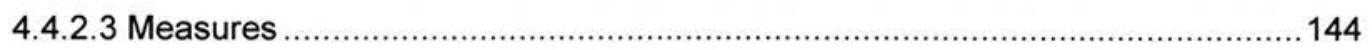

4.5 Recommendations for Future Research.............................................................146

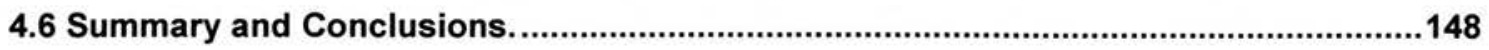

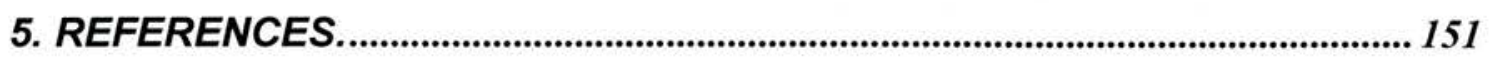

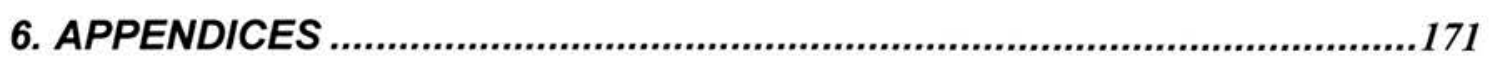




\section{LIST OF FIGURES}

Figure 1: Worldwide Demographic Projections for the Over-60 Population. ................... 3

Figure 2: Estimated Population in Scotland by Age and Sex..................................... 6

Figure 3: Leventhal's Self-Regulatory Model of Illness Behaviour............................... 16

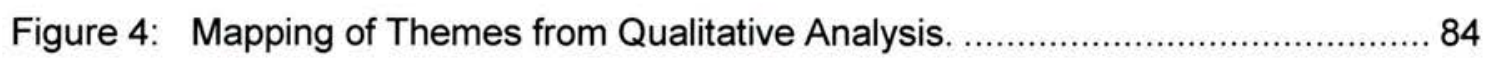

Figure 5: Comparison of Age in Non-clinical and Clinical Groups. .............................. 88

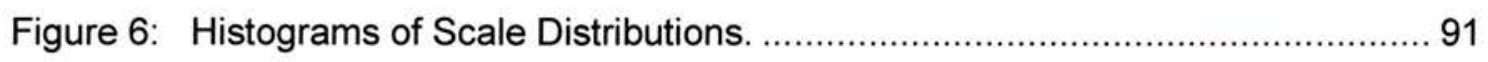

Figure 7: Comparison of Group Mean Scores on BMHSS 'Ageism' Sub-scale. ......... 100

Figure 8: Scatterplots of Correlations of Attitudinal Variables.................................. 108 


\section{LIST OF TABLES}

Table 1: Life Expectancy at Birth for World Regions. ................................................. 4

Table 2: Prevalence of Depression and Depressive Symptoms in Older Adults.......... 21

Table 3: Breakdown of Sample and Participation Rates by Source ............................ 57

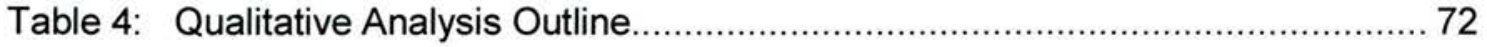

Table 5: Thematic Framework - Attitudes to Mental Illness ...................................... 74

Table 6: Demographic Data for Non-clinical and Clinical Samples............................ 87

Table 7: Mean Age of Non-clinical and Clinical Samples. ....................................... 89

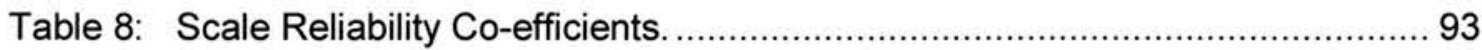

Table 9: Between-Group Comparison of Attitudes to National Survey Items............... 95

Table 10: Comparison of Younger and Older People's Attitudes to Mental Illness. ..... 97

Table 11: Comparison of Young-old and Old-old Attitudes to Mental Illness. ............... 98

Table 12: Comparison of Attitudes to Mental Illness in Later Life by Experience....... 102

Table 13: Comparison of Attitudes to Ageing across Groups. .................................. 104

Table 14: Correlations between Attitudinal Variables.......................................... 107

Table 15a: Regression Model Summary Statistics for BMHSS 'Ageism' as DV. ....... 110

Table 15b: Regression Coefficients for BMHSS 'Ageism' as DV.............................. 111

Table 16: Comparison of Group Scores on RHBC and GHQ-12 .......................... 113

Table 17: Partial Correlations between Health Behaviours, Well-being and Attitudes.114

Table 18a: Regression Model Summary Statistics for RHBC as DV. ........................ 116

Table 18b: Regression Coefficients for RHBC as DV ............................................ 116

Table 19a: Regression Model Summary Statistics for GHQ-12 as DV. ..................... 117

Table 19b: Regression Coefficients for GHQ-12 as DV. ....................................... 117

Table 20: Summary of Between-group Comparisons......................................... 121 


\section{LIST OF APPENDICES}

\section{Appendix 1: Ethical Approval and Requested Amendments}

1.1 Local Research Ethical Committee Approval .........................................................172

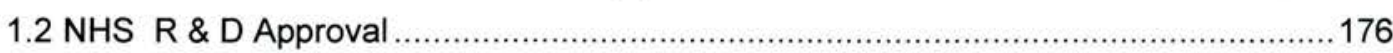

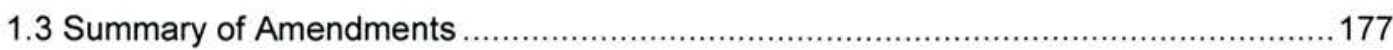

\section{Appendix 2: Study Documents (Examples)}

2.1 Study Invitation Letter. 178

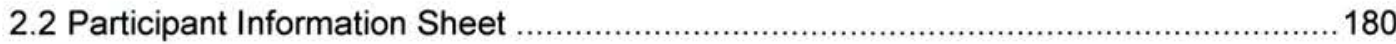

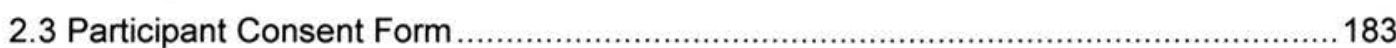

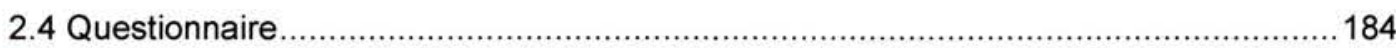

\section{Appendix 3: Focus Group Materials and Qualitative Analysis}

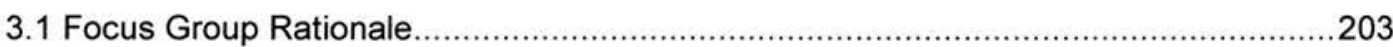

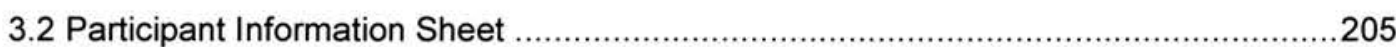

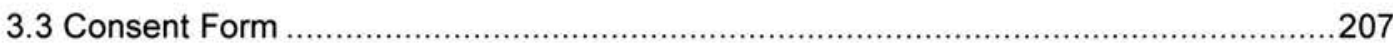

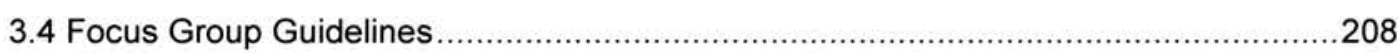

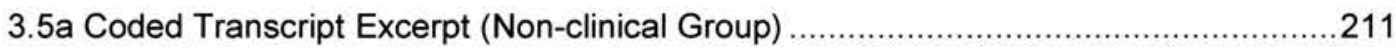

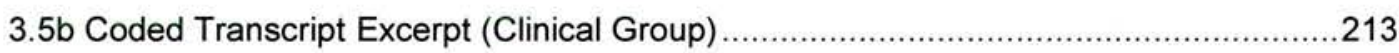

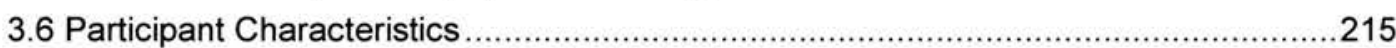

3.7 Proposed Questionnaire Modifications................................................................216

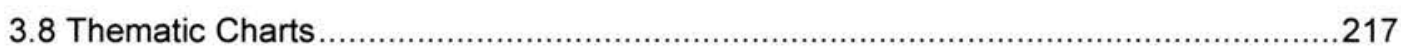

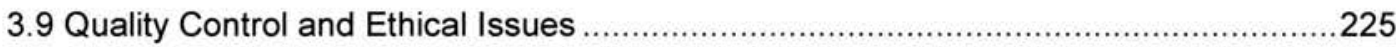

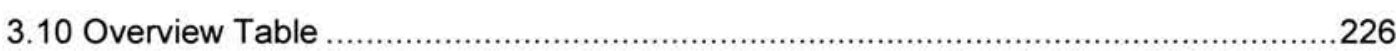

\section{Appendix 4: Quantitative Analysis}

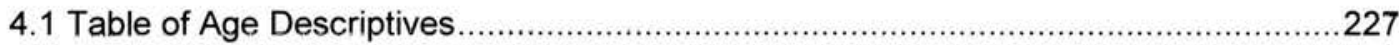

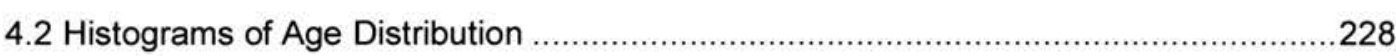

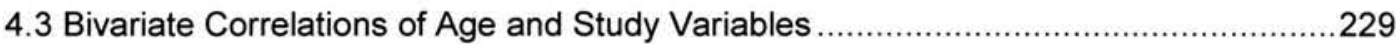

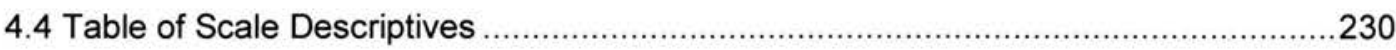

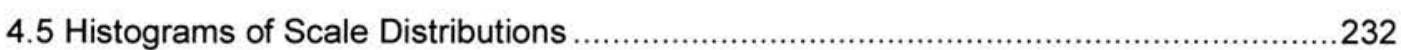

4.6 Tests of Homogeneity of Variance for Computed Scales..........................................239

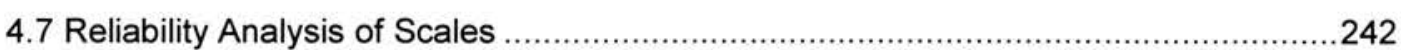

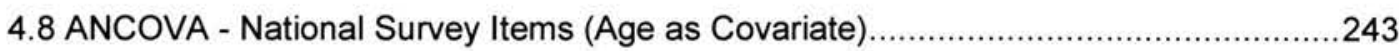

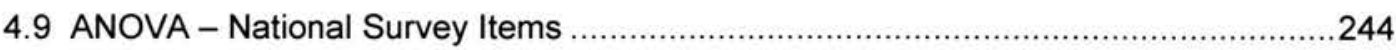

4.10 Comparison of National Survey Item 1 by Gender..................................................245 
4.11 MANCOVA - Attitudes to Mental Illness (Age as Covariate) …...............................246

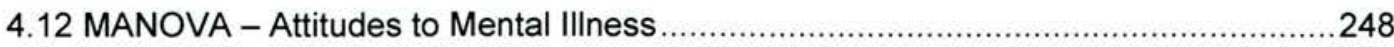

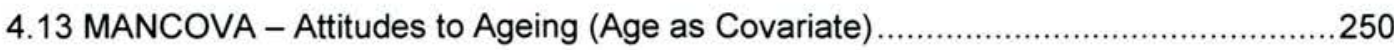

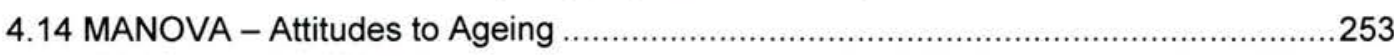

4.15 Boxplots of Attitudes to Ageing Scales by Group ....................................................256

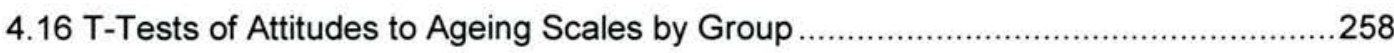

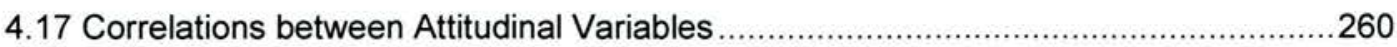

4.18 Multiple Regression Analyses - BMHSS 'Ageism' as Dependent Variable ................262

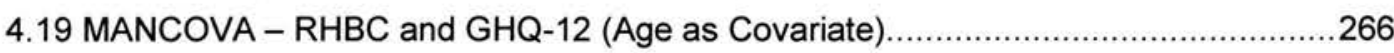

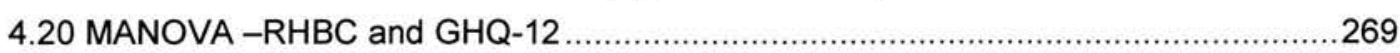

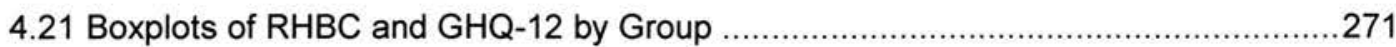

4.22 Correlations between Health Behaviours, Well-being and Attitudes..........................273

4.23 Scatterplots of RHBC, GHQ-12 and Attitudinal Variables .......................................275

4.24 Multiple Regression Analyses - RHBC Total Score as Dependent Variable .............2277

4.25 Multiple Regression Analyses - GHQ-12 as Dependent Variable .............................281 
ABSTRACT

Objectives: Despite the prevalence of mental health problems in later life, older people markedly underutilise mental health services. A greater awareness of factors influencing older peoples' attitudes to mental illness may therefore improve recognition, presentation, and treatment of mental disorders in this population, and so enhance quality of life. This study explores older people's attitudes to mental illness within the context of ageing, and considers whether attitudes act as potential barriers to engagement in health-related behaviours, and lead to lower subjective well-being.

Design/Method: A combined qualitative and quantitative methodology was used. First, focus groups were undertaken to pilot the study questionnaire and explore the subject area. A crosssectional independent groups design was then employed to compare responses of clinical (i.e. current service users) and non-clinical participants on a self-report questionnaire assessing attitudes to mental illness and ageing. 74 completed questionnaires were received ( 24 clinical; 50 non-clinical).

Results: Similar to younger people, older people endorsed a range of positive and negative attitudes to mental illness. However, when attitudes to mental illness were considered within the context of ageing and experience a more complex pattern of results emerged. While negative attitudes to mental illness were associated with negative attitudes to ageing across the entire sample, clinical participants (and those with prior experience of mental illness) reported more positive attitudes to mental illness and more negative attitudes to ageing than non-clinical participants, for whom the reverse was true. Attitudes were also differentially related to outcomes. Thus, positive attitudes to ageing predicted stronger endorsement of health-related behaviours $\left(\mathrm{F}_{(2,71)}=9.93, \mathrm{p}<0.001\right)$, while negative attitudes to ageing and mental illness predicted lower subjective well-being $\left(\mathrm{F}_{(4,67)}=22.99, \mathrm{p}<0.001\right)$.

Conclusions: Attitudes to mental illness and ageing may be linked and mediated by personal experience and capacity for psychological self-regulation in the face of age-associated adversity. As one potential cause of the under-use of mental health services by older people, attitudes to mental illness in later life should form the focus of targeted health education interventions designed to address ageist misconceptions and ensure that all older people are given the opportunity to receive care likely to improve their health and well-being. 


\section{1 - INTRODUCTION}

\subsection{OVERVIEW}

Good mental health is crucial to staying well and active in later life just as it is all other times (Blazer, 2006). While illnesses such as depression and anxiety may be prevalent in later life, much can be done in terms of treatment to alleviate the distressing nature of these conditions, and to address issues of excess disability that may arise when psychological problems occur in the context of co-morbid physical illness. Accordingly, mental health in later life is named as one of six priority areas of the National Programme for Improving Mental Health and Well-Being (Scottish Executive, 2003-2006). Yet much of the focus of this work to date has been directed towards addressing issues of mental health in the general (i.e. younger) adult population with only a limited amount of research being been undertaken specifically in relation to older people.

Stigma against older people who suffer from mental health difficulties may be exacerbated by a 'double jeopardy' of negative societal views of ageing and of mental illness (World Health Organisation-World Psychiatric Association [WHOWPA], 2002, p.9). In addition, older people may themselves hold negative views about their own ageing and about mental health problems reflecting cohort beliefs (i.e. particular beliefs held by a 'birth-year defined age group'; Laidlaw et al., 2003, p.30), internalised ageism (Levy, 2003) and the understandability phenomenon (i.e. the belief that depression is a normal part of ageing; Blanchard, 1992). Negative attitudes and beliefs may lead to under-reporting and misdiagnosis of problems, 
increased physical and psychological morbidity with associated increasing health care costs, poor health-related behaviours, and significant reductions in quality of life. To date, however, little empirical evidence exists to either support or refute the presence or effects of such attitudes. This study therefore aims to assess the views of older people in relation to mental illness with a particular focus throughout upon attitudes towards depression and anxiety in later life, rather than upon mental illness as conceptualised within a broader context. An additional aim of the study is to ascertain in what ways, if any, attitudes reflect broader ageist stereotypes, and/or personal experience of mental illness or mental health care services. The associations, if any, between attitudes, experiences and health-related outcomes will also be explored.

\subsection{THE DEMOGRAPHIC CONTEXT}

\subsubsection{World Population Ageing}

The age structure and composition of the world's population is undergoing an irreversible and profound change (Laidlaw \& Baikie, 2007). Current demographic projections indicate that the proportion of people aged 60 and over is growing rapidly (Figure 1) with the oldest-old section of society (people aged 80 years plus) showing the most dramatic increase (Laidlaw et al., 2003). Between 1970 and 2025, a growth in the number of older persons of approximately 694 million (223 per cent) is expected, resulting in a global population of around 1.2 billion people over the age of 60 (WHO, 2002). 


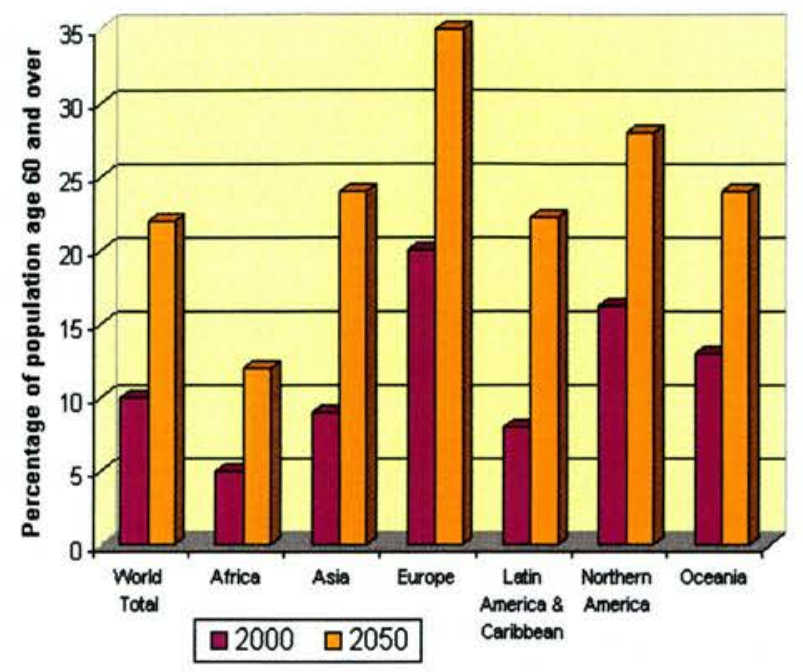

\section{Figure 1: Worldwide Demographic Projections for Over-60 Population}

(Source: UN Population Division - World Population Prospects, 1998)

Patterns of decreasing fertility account for some of this demographic change (Walker \& Maltby, 1997; WHO, 2002). However, despite the decline in fertility, the world's population continues to grow and is expected to increase from 6.5 billion inhabitants in 2005 to 9.1 billion by 2050 . Until relatively recently, population ageing was associated mainly with the more developed regions of the world ${ }^{1}$, where an increase in life expectancy is projected from 76 years currently to 82 years by mid-century. However, among the least developed countries, where life expectancy is generally much lower, an increase is still anticipated from 52 years in 2005 to 67 years in 2045-2050 (Table 1).

\footnotetext{
${ }^{1}$ World regions are classified as more or less developed as follows: More developed regions comprising all regions of Europe plus Northern America, Australia/New Zealand and Japan. Less developed regions - comprising all regions of Africa, Asia (excluding Japan), Latin America and the Caribbean plus Melanesia, Micronesia and Polynesia (UN, 2006).
} 
Table 1: Life Expectancy at Birth for World Regions: 2000-2005, and 2045-2050

\begin{tabular}{lcc}
\hline Major Area & $\mathbf{2 0 0 0 - 2 0 0 5}$ & 2045-2050 \\
\hline World & 66.0 & 75.4 \\
More developed regions & 75.6 & 82.4 \\
Less developed regions & 64.1 & 74.3 \\
$\quad$ Least developed regions & 52.7 & 67.2 \\
$\quad$ Other less developed countries & 66.6 & 76.4 \\
\hline
\end{tabular}

(Source: UN Population Division - World Population Prospects, 2006)

As a consequence of declining fertility and increasing longevity, the world's older adult population is estimated to show a three-fold increase over the next 50 years, with the majority living in developing countries (WHO, 2002). In the U.K. there are currently 9.7 million people aged 65 and over, accounting for approximately onesixth of the total population (Mental Health Foundation \& Age Concern, 2006).

The older population itself is also ageing. In 2000, across Europe, life expectancy at age 60 for men was 17 years, whereas for women it was 22 years. These figures are predicted to change to 21 years and 26 years, respectively, by 2050 (UN, 2001). The oldest-old section of society is therefore expected to increase up to five-fold, with the numbers of people aged over 90 showing an eight-fold increase (Laidlaw et al., 2003). This trend is particularly salient for women, since women account for a higher proportion of older people in general, and especially of the oldest-old age group (Kinsella \& Velkoff, 2001). In the U.K. today there are an estimated 8,100 centenarians, the majority of whom are women. Although the mortality age gap 
between the sexes has fallen over recent years (to between 5-7 years), women are still more likely to outlive men, to be widowed, to live alone, and to experience chronic health conditions such as arthritis, hypertension, osteoporosis and diabetes. They are also more likely to come to the attention of psychiatric services for mental health difficulties such as anxiety and depression (Crawford et al., 1998).

\subsubsection{Ageing in Scotland}

The demographic profile of Scotland reflects global demographic trends. Scotland continues to have a slightly older population structure than the rest of the U.K. due to its lower fertility rates and net outward migration of younger age groups (Wood \& Bain, 2001). The older population in Scotland has therefore been growing in both absolute and relative terms, leading to a projected increase in the number of people aged 65 years and over from 787,000 in 2000 to an estimated 1.2 million in 2031 (Scottish Executive, 2002a). Figure 2 depicts changes in the age/sex composition of the Scottish population. Currently, people of pensionable age make up approximately 19 per cent of the total population. Women substantially outnumber men in the older age groups (particularly those aged over 75) reflecting longer life expectancy for women (currently 79.2 years for females, 74.2 years for males), and higher rates of male mortality during the Second World War (Registrar General for Scotland, 2006). 


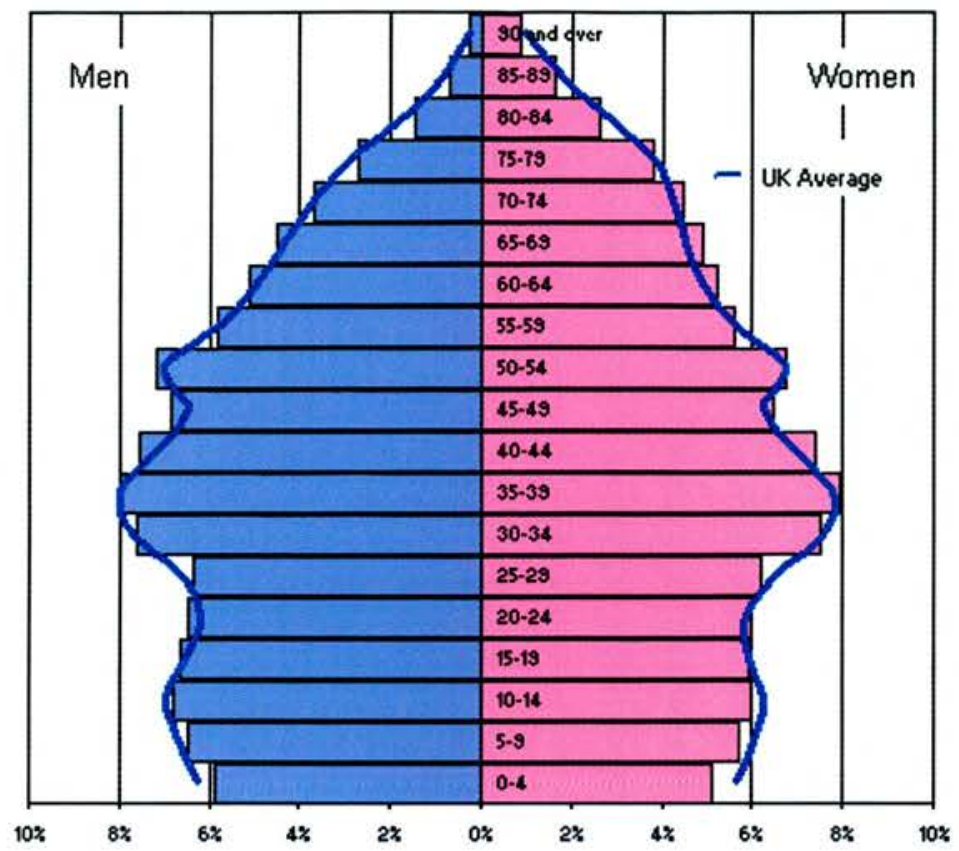

\section{Figure 2: Estimated Population in Scotland by Age and Sex.}

(Source: Census 2001, Population Data)

\subsubsection{Implications of Population Ageing}

It is quite clear from these demographic indicators that we live in an ageing world. While this has been recognised for some time in developed countries, it is only recently that this phenomenon has been fully globally acknowledged. Indeed, population ageing in developing countries is occurring rapidly and at a pace which outstrips the socio-economic development of these world regions. Thus, while population ageing is undoubtedly a significant human achievement, it also poses a complex series of social, political, and economic challenges worldwide (WHO, 2002). For example, as a growing sector of the population, older people are major consumers of health and social care services. In Scotland, for instance, over- $65 \mathrm{~s}$ account for 15 per cent of the population consuming some 40 per cent of health and 
social care spending, with over-75s making the greatest use of services (Scottish Executive, 2002a).

As the relative number of older people living with long term conditions (such as stroke, heart disease, and diabetes) increases, Jeste et al. (1999) argue that there is likely to be a concomitant increase in the number of older people with potentially disabling chronic mental illnesses. This will necessarily increase demand for adequate age-specific health and social care infrastructures which will deliver the types of interventions required to meet both the physical and psychological needs of older people (Laidlaw \& Baikie, 2007). From this perspective, it is no longer possible to ignore the needs of an ageing population, regardless of whether ageing per se is perceived as an inherently positive or negative phenomenon.

\subsection{A SCOTTISH POLICY PERSPECTIVE}

\subsubsection{Background}

In response to the challenges of population ageing outlined above, the Scottish Executive has consistently identified older people as a key target group deserving of improved healthcare within the NHS, and mental health (in the general population) as a clinical priority area. In Scotland, one of the key recommendations of the Scottish Health Plan (Our National Health - A Plan for Action, A Plan for Change; Scottish Executive, 2000), was for the provision of a multidisciplinary expert group to identify and address current failures of health service provision to meet the 
physical and psychological needs of an ageing population. The plan also led directly to the development of a range of policy documents aimed at the promotion of positive mental health and well-being and/or mental health service re-design and delivery, although relatively few of these have combined the two policy agendas of improved mental health and older people.

The report of the expert group (entitled Adding Life to Years; Scottish Executive, 2002a) drew attention to the apparent lack of mental health services to address psychological difficulties in later life. It also outlined the predicament of many older people who potentially required, but were not currently in receipt of, treatment and who therefore 'suffered in silence' (p.39) due to patchy provision and the stigma of mental illness. The general failure of the NHS in Scotland (NHSiS) to adapt to the changing needs of an ageing population was called into question as evidence of 'structural ageism' (p. 44). However, a review of the published literature carried out on behalf of the expert group did not support allegations of widespread ageism, despite acknowledging concerns regarding the existence of ageism (Gilhooly, 2001). As one medical practitioner quoted in the report stated: "sadly the NHS is quite capable of providing poor services for older people without being in the slightest ageist" (Scottish Executive, 2002a, p.52).

To address the mismatch of mental health service provision to the needs of an ageing population, Improving Health in Scotland: The Challenge (Scottish Executive, 2003) made specific provision for the establishment of a three-year National Programme 
for Improving Mental Health and Well-Being (Scottish Executive, 2003-2006), in which mental health difficulties in later life were designated a priority issue.

\subsubsection{The National Programme and Recent Policy}

The National Programme was launched by the Scottish Executive in 2003 to support action in mental health improvement by addressing the stigma of mental illness in Scotland. Older people were identified within the programme as one of six priority groups particularly adversely affected by negative attitudes, ageism and discrimination. To inform the work of the programme, the Executive commissioned the first national survey of public attitudes to mental health in 2002 entitled 'Well: What do you think?' (Scottish Executive, 2002b; 2004), with a view to replicating this survey every two years ${ }^{2}$ in order to track progress towards achievement of programme aims. The objectives of the survey were to ascertain the views of a representative sample of the Scottish adult population on a range of issues including: factors affecting mental health and well-being; experience of mental health problems; awareness of sources of information relating to mental health problems; and attitudes to mental health.

Results from the survey are reviewed in Section 1.5.3.2 and highlight some interesting points in relation to attitudinal variation across the lifespan. However, of particular relevance to the current discussion is the relative lack of emphasis within the published report upon broader issues of mental health in later life. This anomaly

\footnotetext{
${ }^{2}$ Data from the 2006 survey had not been published at the time of writing.
} 
is apparent throughout the national programme despite its reflection of the convergence of political interest in changing demographics and the resource implications of shifting patterns of illness. Thus high-profile campaigns of the national programme aimed at tackling issues of stigma and discrimination, such as 'See Me' (www.seemescotland.org), and those addressing barriers to treatment, such as 'Breathing Space' (www.breathingspacescotland.co.uk), have largely failed to address the needs and views of the older population. Similarly, while some circumscribed funding is available within the remit of the programme to support local work in the areas of increasing awareness and promoting mental health in later life (i.e. NHS Health Scotland's health in later life programme), this appears to be relatively sporadic and lacks a coherent approach. This situation exists despite the identification by older people of prevailing ageist societal attitudes as key factors impacting on mental-health and well-being, treatment-seeking behaviour, and quality of life (Bostock \& Miller, 2003). Moreover, those aspects of the national programme that are specifically directed towards older people tend to focus on raising awareness and provision of support for the dementias rather than the more highly prevalent functional disorders (Mental Health Foundation \& Age Concern, 2006). A focus on dementia is also apparent in the recent policy planning documents Delivering for Mental Health (Scottish Executive, 2006), and All Our Futures (Scottish Executive, 2007). 


\subsubsection{Policy Summary}

The mental health needs of older people have long been neglected, yet as people are living longer so pressure on services will increase (Laidlaw \& Baikie, 2007). At the current time, older people with mental health difficulties appear to fall somewhere between the cracks of two parallel but rarely intersecting policy agendas. While mental health and age-inclusivity are prevalent themes in policy development, they are rarely cross-cutting. Even when these do coincide, the focus is largely upon neurodegenerative conditions rather than upon functional disorders, irrespective of the more highly prevalent and treatable conditions of depression and anxiety in older people, and consequent implications for individual quality of life and health service utilisation. Despite recognition of gaps in service, and rhetoric to the contrary, this is as true of current as it is of past policy documents. Consequently, mental health services for the aged remain fragmented, inconsistent, and subject to widespread geographical variation.

\subsection{MENTAL HEALTH PROBLEMS IN LATER LIFE}

\subsubsection{Factors Affecting Recognition, Diagnosis and Treatment}

Mental illness in older people is a significant and serious issue (Blanchard, 1992; Woods, 1999). Although marked variation exists in terms of community-based prevalence rates (Blazer, 2003), there is a general consensus within the empirical literature that depression and anxiety have a significant impact on an individual's quality of life and experience of ageing, and are frequently under-reported and under- 
detected (Beekman et al., 1999; Birrer \& Vemuri, 2004; Blazer, 2003). Symptoms of depression and anxiety in the elderly are also associated with significantly higher health care costs, even after adjustment for co-morbid chronic physical illness (Katon et al., 2003). Kramer (1983) has referred to this phenomenon as the 'rising pandemic of mental disorders' (p.115). In this context, it is therefore somewhat surprising that psychiatric illness in later life remains a relatively under-researched area.

In part, this noted lack of research may be explained by the complexity of issues facing prospective researchers and clinicians working in this area. For example, psychiatric illnesses in later life may have multiple aetiologies reflecting the relative heterogeneity of the older adult population (Laidlaw et al., 2003), and posing an array of challenges for health care professionals. In 1964, Williamson and colleagues published findings indicating that general practitioners knew relatively little about the mental health needs of older patients. This situation appears to have improved little over the years. For example, Crawford et al., (1998) reported that GPs were aware of depression in just over one-half of 70 depressed patients aged over 65 , and it is still likely that a significant number of older people with mental health problems do not come to the attention of mental health services (Blazer, 2002; de Beurs et al., 1999).

There are several reasons why psychiatric illness may not be recognised in general practice. For example, the presence of physical co-morbidity in the form of chronic health conditions such as diabetes or arthritis may influence or mask the expression 
and recognition of mental health difficulties such as depression. Thus, in many cases, symptoms of psychological problems may go undetected by health professionals treating older people for what they perceive as primarily physical complaints (Blanchard, 1992; Laidlaw et al., 2003). Providers may fail to detect or treat mental health difficulties due to lack of awareness or specialist geriatric/gerontological training. Providers may also be reluctant to inform older patients of a diagnosis of depression due to uncertainty about diagnosis, reluctance to stigmatise, pessimism about therapeutic outcome, concerns about polypharmacy or lack of access to appropriate specialist care (U.S. Department of Health and Human Services, 1999). Ageism, both structural (Scottish Executive, 2002a), and internalised (Levy \& Banaji, 2002) may also account for the relative neglect of this group of patients within the mental health service sector of the NHS.

From the patient's perspective, Blanchard (1992) argues that older people may hold beliefs regarding the perceived legitimacy of physical symptoms, where such symptoms are considered necessary in order to make an appointment with the doctor. They may also under-report symptoms due to misattribution (e.g. attributing psychological problems to physical illness), cohort beliefs (Knight, 1999; Laidlaw et al., 2003), perceived barriers to accessing treatment (Pepin, 2006), and social stigma:

"the stigma of mental illness is especially strong in the current cohort of elderly people who tend to associate mental disorder with personal failure, spiritual deficiency, or some other stereotypic view. Thus, the need for mental health treatment is not accurately or appropriately recognised (or is denied) by patients, members of their families, and even the primary care physicians who provide most of the care to the elderly". (Lebowitz \& Niederehe, 1992, p.17) 
Socio-cultural stereotypes relating to the ageing process may result in the assignation of depressive symptoms, such as withdrawal or low mood, to a normal part of the ageing process. Societal perceptions of mental illness in old age (i.e. the so-called 'double stigma' effect; Sartorius, 2003) may influence an individual's attitude to their own ageing and mental health, thus impacting on treatment-seeking behaviour. This may in turn influence an individual's experience of later life and so represent a significant obstacle to optimal ageing.

\subsubsection{Health and IIIness Beliefs}

The ways in which people think about health and illness may also impact significantly on health-related behaviours such as help-seeking, coping, and adherence with treatment (Lobban et al., 2003; Ogden, 2000). Health and illness beliefs form the core of many current models of health psychology, and have been studied extensively in relation to the role of psychological processes in physical illness (particularly chronic illnesses such as stroke, diabetes, and cancer; Ogden, 2000). However, specific beliefs about mental illness have rarely been considered in the empirical literature, and even less so in relation to older people.

The way in which an individual labels an illness experience (i.e. whether they consider it as illness-related) may have an important impact on outcome. For example, it does not seem unreasonable to propose that individual beliefs about health and illness could also impact upon symptom perception and treatment-seeking in relation to psychological difficulties much as they do in physical illness. 
Moreover, as Lobban et al. (2003) argue, the development of psychological theories and models common to both physical and mental health may facilitate a more integrated approach in which mental illness becomes less stigmatised within treatment settings. One example of a model with potential utility in this area is the self-regulation model (SRM) of illness behaviour (Leventhal et al., 1992).

The SRM of illness behaviour has established reliability and validity in exploring patient beliefs across a range of physical illnesses. Lobban et al. (2003) suggest that the widespread applicability of the model indicates that it may also be appropriate for mental as well as physical illness, while Leventhal et al. (1992) have also briefly described how the model can be usefully applied to mental illness. In the SRM, beliefs about illness (or 'illness representation'; Leventhal et al., 1997), for example, about the perceived cause, controllability or consequences of an illness, are proposed to play a significant role in symptom perception since they provide the individual with a possible framework within which to understand illness. Deviations from normality are proposed to alert the individual to possible signs and symptoms of illness, and motivate the individual to address problems through a process of interpretation (making sense of the problem), coping (selecting a strategy for dealing with the problem) and appraisal (evaluating the success of the strategy) (Figure 3). Self-regulation involves the dynamic interaction of these three processes and represents an attempt by the individual to return to a normal state of health (i.e. to regain homeostasis). 


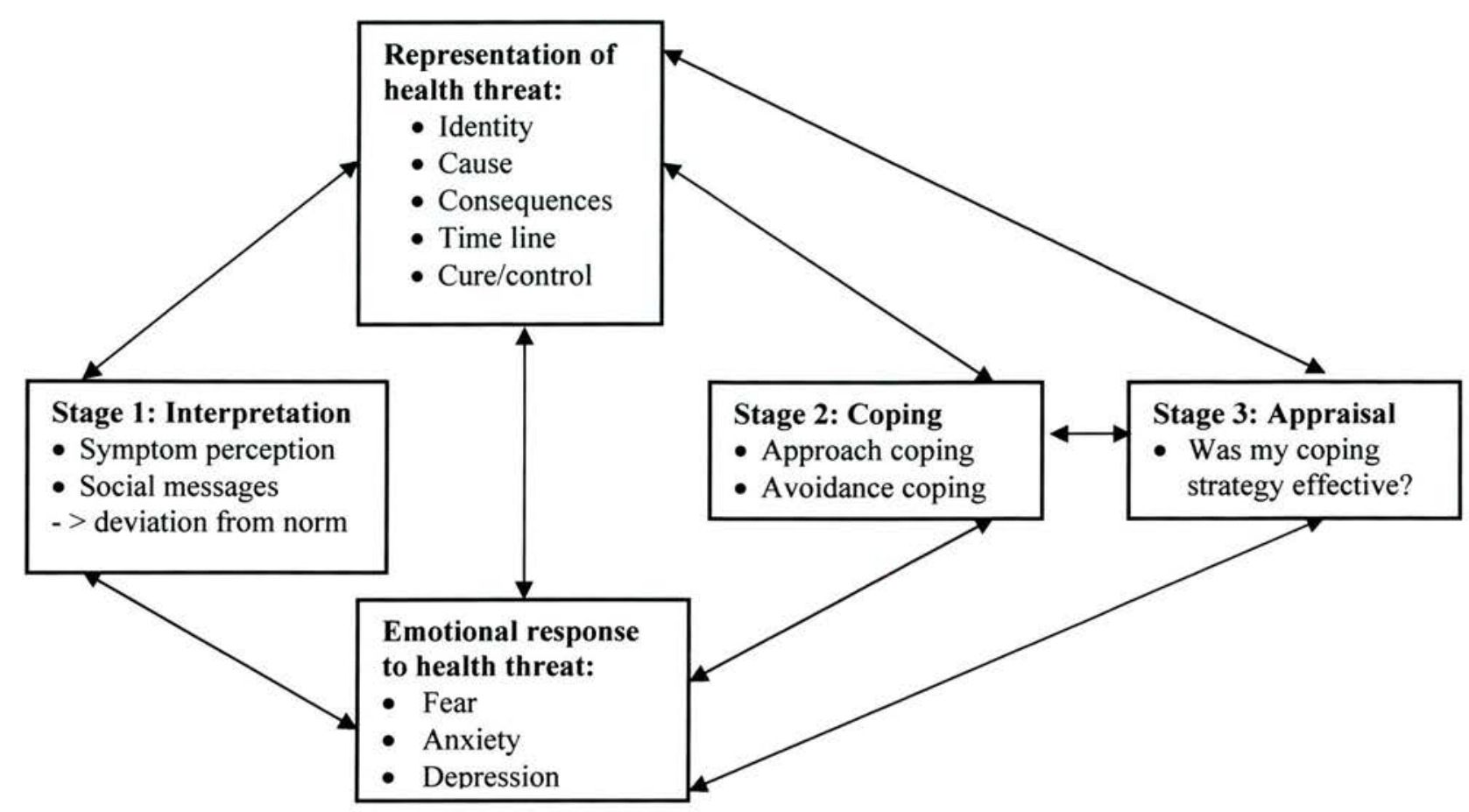

Figure 3: Leventhal's self-regulatory model of illness behaviour

(Source: Ogden, 2000, p.48)

The concept of active homeostasis in the face of physical or psychological threat is particularly relevant to discussions of ageing. As indicated earlier, individuals are faced with a variety of challenges as they grow older (e.g. development of chronic health difficulties; multiple losses etc.). People who are considered to make the most successful adaptations to the experience of ageing are those who consider ageing as just another phase of life and maintain optimal levels of functioning by minimising the impact of negative experiences (i.e. maintaining a degree of harmony and balance between the losses and gains of ageing) (Laidlaw \& Baikie, 2007). This view is encapsulated in the theory of selective optimisation with compensation (SOC) introduced by Baltes \& Smith (2002), which focuses on maintaining function in later life when limits of capacity are reached or superseded. The theory suggests that 
older people cope with reduced, or loss of, capacity by compensating for losses through selection (of desired goals), optimisation (focusing resources on achievement of specific goals), and compensation (engaging alternative means of achieving the highest possible level of functioning). SOC thus represents a psychological self-regulatory approach to life management which fits well with the SRM model of active homeostasis. However, while the SOC model has been specifically developed to address the experience of ageing within the context of the physical and psychological challenges of later life, Lobban et al. (2003) argue that modifications to the SRM are likely to be necessary for application of the latter model beyond the study of health beliefs and physical illness in the general population.

The potential applicability and relevance of models of self-regulation to the study of attitudes to mental illness in later life is considered in more detail in Chapter 4 (Sections 4.2.2 and 4.2.3).

\subsubsection{Depression in Later Life}

Of all the mental health problems affecting older people, depression is perhaps the most prevalent, associated with serious morbidity and mortality from both natural and non-natural causes (Blazer, 2003; Chew-Graham et al., 2004). In late life, the course of depression tends to be more chronic than in younger adults (Cole \& Bellavance, 1997), relapse rates are higher, and a substantial number of older people do not recover with the standard treatments. Depression may also occur co-morbidly with chronic physical health conditions such as stroke (Kneebone \& Dunmore, 2000) 
and with other mental health conditions, particularly anxiety (Beekman et al., 2000). The course of depression and its interaction with treatment may be influenced by a number of factors in later life, highlighting the importance of research targeted at older age groups rather than reliance on extrapolations from younger patients (U.S. Department of Health and Human Services, 1999). Yet, in practice, older people may be disadvantaged by clinical research studies in which they are excluded due to the imposition of arbitrary age cut-offs. Indeed an estimated 15 per cent of trials exclude older people in this manner, with resulting implications for the utility of clinical trial data in the design and implementation of older peoples' services (McMurdo et al., 2005).

\subsubsection{Aetiology and Onset}

Possible aetiological factors in depression include biological and genetic susceptibility, psychosocial factors and life stressors, with certain risk factors becoming more pronounced at different stages of life (for example, biological vulnerability appears to increase markedly in later life while psychological vulnerability decreases; Karel, 1997). The first onset of a major or minor depressive episode can occur early in life (i.e. early onset depression: EOD) or in old age (i.e. depression occurring de novo after age 60 - termed late onset depression or LOD; Seymour, 2006), although this distinction is somewhat problematic and misleading and may reflect methodological shortcomings in clinical research. For example, older people categorised as LOD in clinical trials may in fact have experienced depressive episodes earlier in life for which they may not have sought treatment. 
Recent evidence from a community sample has also indicated no clear differences in terms of aetiology and clinical presentation between the two types (Janssen et al., 2006). In 129 depressed elders, LOD was weakly associated with being older, being widowed and having impaired cognition, while EOD was associated with double depression, i.e. major depression superimposed on dysthymia (chronic but milder depression) and more anxiety. Family psychiatric history (indicative of genetic susceptibility), vascular pathology and stressful early and late life events did not differ between groups (Janssen et al., 2006). This is an important finding since it highlights the fact that all older people with depression should be treated with equal rigour regardless of proposed time of onset.

\subsubsection{Prevalence of Depression in Later Life}

Clinical experience suggests that depressive disorders are common in old age, however, prevalence estimates in this population are problematic due to methodological issues. For example, variation in prevalence and incidence rates ${ }^{3}$ of depression across studies reflect differences in case definition, diagnostic methods and instruments, and sampling methodologies (e.g. Blazer, 2003). In general, studies using the Diagnostic and Statistical Manual of Mental Disorders (DSM-IV; $4^{\text {th }}$ Edn., APA, 2000) diagnostic criteria of 'major depression' (defined as presence of low mood and/or anhedonia, together with four or more additional symptoms present for at least two weeks) report lower prevalence and incidence rates of depressive disorders in older adults than those measuring depressive 'symptoms'. Thus,

\footnotetext{
${ }^{3}$ Prevalence measures how much there is of a condition or disease in a population at a particular point in time; incidence refers to rate of occurrence of 'new' cases in a specified time period.
} 
prevalence rates of major depression in community samples of older people tend to be quite low, ranging from around one per cent to four per cent, with higher prevalence reported among women, and in those of low-socioeconomic status (Beekman et al., 1999; Blazer \& Williams, 1980; Blazer et al., 1987). The large and widely-quoted multicentre Epidemiological Catchment Area (ECA) study (Weissman et al., 1988), reported relatively low point-prevalence of virtually all psychological disorders among older adults, including major depression. The one year prevalence rate for major depression among some 5000 persons aged 65 or over was 1.0 per cent ( 0.4 per cent for men, 1.4 per cent for women) compared with 2-3 per cent and 3-4 per cent for the 45-64 and 18-44 years age-groups respectively, suggesting that clinically significant depression is far less common in late life. However, the ECA study's methodology has been criticised for, among other things, under-representation of older people, misdiagnosis of depressive illness, and exclusion of cases of depression with co-morbid physical illness (Snowdon, 1990).

In contrast to findings from community studies of major depression, reported rates of depressive symptoms, dysthymia and minor (i.e. subthreshold) depression are somewhat higher. A systematic review of 34 community based studies found an average prevalence for depressive syndromes of 13.5 per cent in adults aged over 55 , although reported rates were found to vary enormously between 0.4 per cent and 35 per cent, with an average prevalence of 10.2 per cent (Beekman et al., 1999). The majority of studies report rates between 8 per cent to 17 per cent in communitydwelling samples (see Table 2), with a degree of comparability found across countries and cultures (Chew-Graham et al., 2004), although this finding is not 


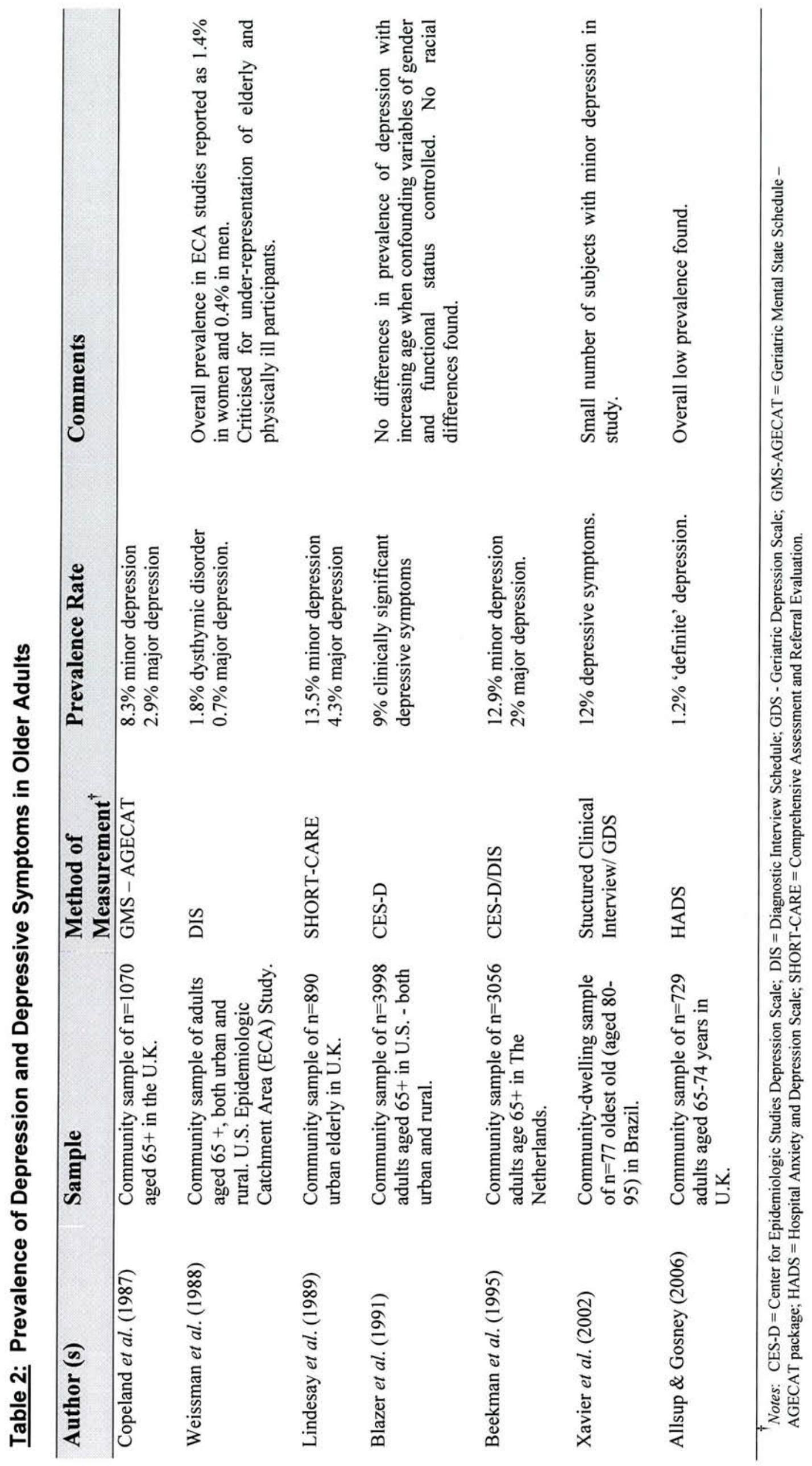


consistently upheld (e.g. Copeland et al., 1999; Minicuci et al., 2002). As with major depression, rates of depressive symptoms are generally higher in women (Beekman et al., 1999), in physically compromised populations (i.e. those with comorbid chronic health conditions), and in those in long-term residential care (Blazer, 2003).

There are several implications of these apparently conflicting results. If, as the ECA and other community studies suggest, depressive disorders are less common in later life, this may impact on decisions of resource allocation, and may increase the likelihood that depression in older people will be missed by medical practitioners who are less alert to the possibility of depressive symptoms in their elderly patients. On the other hand, dementia, disability, chronic physical illness, and bereavements are all much more common in later life, which does not appear to fit with the fact that depressive disorders do not also increase with age (Snowdon, 1990). Interestingly, Baltes and Smith (2002) provide a useful explanation of the observed differences in prevalence rates of depression in the elderly. Thus they argue that while rates of depression are likely to be reasonably low in the young-old, these will increase markedly in the fourth age (age 80 plus years) reflecting the relative vulnerabilities of individuals at this stage in the life-cycle. 
An alternative explanation for the discrepancy between rates of depressive disorders and depressive symptoms noted in the literature may be that many studies underestimate the true prevalence of depression in later life. Thus, it is estimated that almost two-thirds of older people with clinically significant depressive symptoms do not meet current diagnostic criteria for classification of a mood disorder, which have been developed primarily to reflect symptoms in younger people (Chew-Graham et al., 2004). For example, compared with younger depressives, older people who manifest depression for the first time in later life may under-report affective and psychological symptoms such as dysphoria and anhedonia, tending instead to emphasise more somatic complaints such as disturbed sleep and appetite, agitation, aches and pains, and fatigue (Blank et al., 2001). This has resulted in the identification of specific subtypes of depression in later life, including a syndrome of 'depression without sadness' (Blazer, 2003, p. 250), or 'nondysphoric depression' (Gallo et al., 1997, p.570) thought to be more common in older people, as well as a depletion syndrome characterised by withdrawal and apathy (Fogel \& Fretwell, 1985). However, and perhaps most importantly, these appear to result in no less emotional distress or functional disturbance than the more common presentation of depression with low mood (Gallo et al., 1997).

\subsubsection{Presentation and Management}

As discussed, there are therefore several plausible reasons why older people and their relatives/carers may not recognise or appreciate the significance of depressive symptoms. As a result of cultural stereotypes many of the primary symptoms of 
depression, for example, feeling unhappy, becoming withdrawn or becoming forgetful, may be viewed as part of the natural ageing process and may not be reported to health professionals. For those that do present, the 'understandability phenomenon' (i.e. the idea that depression is an expected and normal part of ageing; Blanchard, 1992, p253) may contribute to a sense of 'therapeutic nihilism' (ChewGraham et al., 2004, p.181) on the part of both general practitioners and therapists regarding responsivity to treatment and outcome. Such therapeutic pessimism reflects the legacy of Freud's assertion that older people lack the mental plasticity to engage successfully in therapy (Knight, 1999; Laidlaw et al., 2003). The situation is further complicated by issues of differential diagnosis since depression in later life also characteristically presents co-morbidly with other physical and psychiatric conditions, further hindering detection and management. For example, depression is commonly associated, particularly in the oldest old, with stroke (Kneebone \& Dunmore, 2000), coronary heart disease (Birrer \& Vemuri, 2004), anxiety (Flint, 1997), alcohol/substance abuse (Birrer \& Vemuri, 2004), and cognitive impairment/dementia (Woods, 1999).

The substantial consequences of untreated depression, both in terms of emotional suffering and increased health care costs, are well-documented (Katon et al., 2003; Rabins, 1996). Yet, paradoxically, older people with depression are less likely than any other age group to receive appropriate care (Blank et al., 2001), with as many as 90 per cent not seeing a specialist (Chew-Graham et al., 2004). This is particularly concerning since this age group (and particularly older men) are at the highest risk of committing suicide, with depression, and more specifically, hopelessness, a 
predominant factor in suicide attempts (Woods, 1999). Depression, when detected, tends to be treated pharmacologically, despite a growing (although not wholly uncontested) evidence base for the psychosocial management of late life depression, such as cognitive-behavioural therapy (CBT) or interpersonal therapy (IPT) (e.g. Hinrichson, 2000; Pinquart et al., 2006; Scogin \& McElreath, 1994; Scogin et al., 2005). Thus, there is evidence to suggest that between 10 and 40 per cent of depressed older patients are given medications (Birrer \& Vemuri, 1994), even though older people themselves may be distrustful of tablets, may be prone to the effects of polypharmacy and serious side-effects (Resnick, 2003), and may express a preference for psychological interventions (Rokke \& Scogin, 1995) which remain largely unavailable to them.

\subsubsection{Summary and Implications}

The above discussion highlights the fact that depression is an eminently treatable condition in later life and emphasises the need to better understand possible barriers to detection and treatment in this population. Improvements in these areas may provide relief from the profound suffering of elderly depressed patients as well as much needed amelioration of the excess disability (i.e. 'the proportion of a person's disability that is not determined by the physical impairments of the condition', Laidlaw et al., 2003, p.11) that can result from physical and psychological comorbidity. Psychological health is essential for the maintenance of well-being, successful ageing and quality of life in old age, and depression remains a prevalent psychiatric illness in older people (Blazer, 2003). Several reasons for the under- 
detection and under-treatment of this disorder have been proposed from the perspectives of both the older person and health professional. Negative stereotypes, stigmatising attitudes, ageism, and therapeutic pessimism continue to act as impediments to treatment-seeking and provision of appropriate care for older people with mental health difficulties (Laidlaw et al., 2003).

\subsubsection{Implications for Health Service Utilisation and Current Research}

The somatic presentation of depression and other mental health disorders in later life, and reported barriers to the effective detection, treatment and provision of appropriate specialist care for mental health difficulties in older people, result in significant additional costs to the NHS. Depression in later life, for example, is associated with increased use of hospital and outpatient medical services, and increased amounts of nursing care for those in residential settings (Blazer, 2003). In a study of 11786 adults aged 60 years and older attending primary care clinics in Seattle, depression was associated with significantly higher health care costs, even after adjustment for chronic medical illness (Katon et al., 2003). Overcoming obstacles and increasing accessibility to specialist mental health services for older people with mental health difficulties thus has widespread personal, societal and financial implications. Of all the factors that could potentially act as barriers to meeting the mental health needs of older people, negative attitudes to mental illness and ageist misconceptions are perhaps the most prevalent and destructive (Currin et al., 1998; Levy, 2003; Pepin, 2006). 


\subsection{ATTITUDES TO MENTAL ILLNESS}

\subsubsection{Introduction}

While there is evidence to suggest that society holds negative attitudes in relation to mental illness (e.g. Crisp et al., 2005), and to mental illness in older people in particular (e.g. Connery \& Davidson, 2006; Sartorius, 2003), little is known empirically about the perceptions of older people themselves. If, as some studies have suggested (e.g. Lundervold \& Young, 1992), older people endorse more negative views of mental illness (both generally, and in relation to mental illness in later life), it is unclear to what extent these views are inconsistent with the beliefs of younger adults. One should also be wary of implicit assumptions of homogeneity of the older adult population since there may be at least two generations contained within this age group (Zeiss \& Steffen, 1996), and differences may realistically be observed, for example, in the young-old (65-79) and old-old cohorts $(80+)$ (Baltes \& Smith, 2002). These points are addressed in more detail in the following sections.

\subsubsection{Stigma of Mental IIIness}

\subsubsection{Stigma and Shame}

The term 'stigma' refers to the negative effects of a label placed on a specific group, and has grown out of sociological theories of deviance and labelling (e.g. Goffman, 1968; Scheff, 1966). According to labelling theory, children learn negative stereotypes at an early stage which are perpetuated over time by society, experiences and the media. Many studies have shown that stigmatising attitudes towards people 
with mental illness are widespread (Crisp et al., 2000; Hook \& Andrews, 2005; Jorm et al., 1997) and play a negative role at almost every stage of mental illness from recognition to outcome (Byrne, 2001). The United States Surgeon General's Report on Mental Health (U.S. Department of Health and Human Services, 1999) described the impact of stigma as follows:

"Stigma erodes confidence that mental disorders are valid, treatable health conditions....A consequent inability or failure to obtain treatment reinforces destructive patterns of low self-esteem, isolation, and hopelessness. Stigma tragically deprives people of their dignity and interferes with their full participation in society. It must be overcome”. (pg.viii; Executive Summary)

A number of factors including age, gender, and experience may affect levels of stigma and shame associated with mental illness, which may in turn contribute to denial of symptoms, treatment non-compliance, or failure to attend appointments (Byrne, 1997).

\subsubsection{Prevalence of stigmatising attitudes}

In a review of the literature in this area, Hayward and Bright (1997) concluded that there were several enduring themes held in relation to people with mental illness including: perceived dangerousness, unpredictability, being difficult to talk to, having only themselves to blame, not being able to 'pull themselves together', having a poor outcome, and responding poorly to treatment. Gilbert (2000) considers that such perceptions reflect an enduring human propensity to stigmatise those who are different, suggesting that negative attitudes will be prevalent across the lifespan and difficult to change. This view is supported by the results of national 
surveys implemented both in the U.K. and abroad which highlight the prevalence of negative attitudes to mental illness in both younger and older people, and of the effects of educational campaigns which have tended to produce only small and shortlived attitudinal changes. For example, a national survey of Australians reported on the inherent negativity of the general public's knowledge and attitudes to treatment of mental illness (Jorm et al., 1997). Similarly, a North American study comparing the attitudes of younger and older adults towards mental health services and intervention found that the majority of Americans held negative perceptions of the mentally ill, regardless of age, although those in higher social classes had a more positive attitude towards the patient and treatment (Lasoski \& Thelen, 1987).

In the U.K., a similar pattern of transient attitudinal changes in response to educational campaigns has been reported. For example, despite initial indications of favourable changes in public attitudes following the Defeat Depression Campaign (1992-1996) mounted jointly by the Royal Colleges of Psychiatrists and General Practitioners (Paykel et al., 1997), follow-up surveys have demonstrated that negative and stigmatising views remain prevalent (Crisp et al., 2000). Similar findings were also obtained following the Changing Minds campaign, a five-year strategy initiated by the Royal College of Psychiatrists to reduce the stigma of mental illness (Crisp, 1999; James, 1998). A follow-up survey indicated a pattern of responses similar to those obtained pre-campaign, although some small significant changes were also identified in terms of general reductions in the percentages of stigmatising opinions (Crisp et al., 2005). In Scotland, favourable changes in public attitudes toward mental illness have been reported as a result of the 'See Me' anti- 
stigma campaign (Scottish Executive, 2003-2006), although it is too early to tell whether these will be maintained over time. In addition, as noted earlier, 'See Me' does not specifically target stigma associated with mental health difficulties in later life.

\subsubsection{Double-stigmatisation}

The presence of stigmatising and negative attitudes towards mental illness appears to be particularly heightened in relation to older people. In a recent survey of 900 older people (The U.K. Inquiry into Mental Health and Well-Being in Later Life; Mental Health Foundation \& Age Concern, 2006), discrimination on the basis of age was perceived as the most common type of prejudice experienced by people aged 55 and over, and considered to exert a negative effect on mental health. In particular, the stigma attached to mental illness is considered to multiply the difficulties for older people with mental health problems through double stigmatisation (Sartorius, 2003), or exclusion due to mental illness and old age. Double stigmatisation is considered to be one of the most important obstacles to the improvement of quality of life, health and social services for older people with mental health difficulties, and is the focus of a technical consensus statement produced by the Old Age Psychiatry Section of the World Psychiatric Association and the World Health Organisation (WHO-WPA, 2002). The statement acknowledges the presence of stigma in relation

to younger adults with mental disorders, however, it emphasises the impact of 'double jeopardy' (p.9) for older people with mental health difficulties indicating that both issues need to be addressed in any anti-stigma strategies for this age group. 
Certainly if there is a stigma against the mentally ill and older people, it is reasonable to conclude that the most acute impact will be felt by older people utilising mental health services for whom the effects of stigma are likely to be exacerbated (Hayward \& Bright, 1997).

\subsubsection{Cohort Effects and Inter-generational Differences}

\subsubsection{The Views of Older People}

While the presence of negative societal attitudes towards mental illness is widely acknowledged, the specific views of older people towards mental illness are less well known. Studies of the attitudes and beliefs of older people towards mental health disorders have reported inconsistent results, with differences in part attributable to a broad range of methodologies (i.e. questionnaires, door-to-door interviews, telephone interviews, vignettes etc.), and to lack of appropriate comparison groups (i.e. younger adults). In general, findings suggest that older people, particularly those of a lower educational level and social class, are less favourable towards the mentally ill, while exposure to those who are mentally ill leads to more positive attitudes (Hayward \& Bright, 1997). A review of the literature suggests that, among other things, fear, attribution of responsibility, expectations of poor prognosis, and social distancing may account for the prevalence of negative attitudes in the older adult population. For example, in a study of attitudes to mental illness in a community sample in the U.K., Brockington et al. (1993) found that fear of those with mental illness was related to increased age, lower occupational class, and lack of acquaintance with mental illness. 
Other studies have produced results indicating that older people also hold negative attitudes towards mental health services. For example, Lundervold and Young (1992) developed a questionnaire to assess the attitudes and knowledge of 50 older adults towards mental health services and found essentially negative attitudes and knowledge deficits, but provided no comparison data. Similarly, an early study of help-seeking behaviour for psychiatric symptoms in an urban sample of 88 community-dwelling elders by Waxman et al. (1984) reported low rates of current and anticipated mental health service use (only 3 per cent of the sample had seen a psychologist or psychiatrist in the past year; 60 per cent indicated they would not seek professional help in the future for symptoms of depression), a preference for general practitioners as treatment providers, and a low opinion of the effectiveness of mental health professionals. An association was observed between endorsement of negative attitudes and poor help-seeking behaviour, and professional bias or ageist attitudes on the part of service providers were considered less of an obstacle to service use than the older person's own biases and fears about mental illness and mental health professionals (although interaction between barriers was not assessed).

Interpreting their results, Waxman et al. (1984) suggest the influence of broader cohort factors (i.e. the beliefs and attitudes which distinguish a particular cohort from others born at different times; Knight, 1999). Thus, while social views of mental illness may now be more liberal than in previous years, such progressive attitudes were not characteristic of the first half of the $20^{\text {th }}$ century when the current cohort of older adults were forming their own views of mental health and mental illness. Simply put, the attitudes of the current cohort of older adults may reflect belief 
systems that are out of step with modern societal attitudes. The importance of understanding older people in terms of their generational cohort has long been recognised (Knight, 2004), and cohort factors have been proposed to account for attitudinal differences observed between younger and older age groups in many other studies (Laidlaw et al., 2003).

\subsubsection{Results from Comparative Studies}

In the National Scottish Survey of public attitudes to mental health issues ('Well? What Do You Think?'; Scottish Executive, 2002b, 2004), age was not strongly correlated with attitudes, however older people (and particularly those over the age of 75) were reported to hold more negative views. In addition, attitudes were found to vary significantly by personal experience of mental ill-health (generally more positive with experience) and education (generally more liberal with higher education), although the pattern of associations was not straightforward. Responses from the 2004 survey of 1401 participants (aged 16-75 plus) indicated that, in comparison to younger age groups, respondents aged $75+(n=155)$ were more likely to agree with statements which directly stigmatised, or acquiesced in stigmatising, mental illness (e.g. 'The public should be better protected from people with mental health problems'; 'I would find it hard to talk to someone with mental health problems'; 'People with mental health problems are often dangerous'; 'People with mental health problems are largely to blame for their own condition'). Older participants were also less likely to report knowing someone with a mental health problem, and less likely than younger respondents to overestimate the prevalence of 
mental health problems in the general population. Risk factors for negative attitudes (e.g. social isolation, low income, poor health etc.) were also identified as more prevalent in the older adult group.

However, older people were also more likely to agree that 'People are generally caring and sympathetic towards people with mental health problems', and to indicate that the majority of people with mental health problems eventually recover. These views are in line with those reported by Brockington et al. (1993) who found that increased age was associated with greater fear but also greater benevolence. They may also reflect a general softening of public attitudes towards mental illness reported to occur between the 2002 and 2004 surveys, although this change was acknowledged as a potential short-term fluctuation. While these results are interesting, they should be interpreted cautiously since the study was restricted to a community based and relatively healthy sample, and data were not reported for the 55-74 age year group.

In the literature there are some counter-indicators to the view that older people hold more negative views towards mental illness than the general adult population, with several studies failing to identify any intergenerational differences. For instance, Segal et al. (2005) compared the views of younger and older people with respect to beliefs about mental illness and willingness to seek help, and found surprisingly similar views. Participants, who included 79 community-dwelling older people (mean age 75.1 years) and 96 undergraduate students (mean age 20.6 years), completed the Beliefs Toward Mental Illness Scale (Hirai \& Clum, 2000) and the 
Willingness to Seek Help Questionnaire (Cohen, 1999). Older people had generally similar perceptions of mental illness as younger people although they were more likely to consider those with mental health difficulties as being socially unskilled, embarrassing, and socially disagreeable. In contrast to findings by Waxman et al. (1984), older people did not report a lower willingness to seek psychological help, with both groups indicating an openness towards help (willingness levels were in fact quite high for both age groups). These results suggest that older adults' perceptions of mental illness may not be as different from those of younger adults as previously thought.

A recent study in the U.S. by Pepin (2006) assessing barriers to mental health care also found few differences between younger and older adults, with both groups expressing similar views of stigma and help-seeking. In contrast to expectations, younger participants perceived practical issues of insurance and finding a therapist as more problematic, while older participants found concerns about therapist's qualifications to be a larger barrier (perhaps reflecting lack of familiarity with mental health services in this population). Barriers were also found to interact, for example, negative beliefs about mental health care coupled with patchy provision of specialist care.

While the above studies suggest that older and younger adults may hold similar views in relation to mental illness, attitudes may actually be becoming more positive among the elderly, with evidence of a 'positive cohort shift' in attitudes toward mental health services (Currin et al., 1998, p.506). Comparing the views of two 
independent samples of older adults recruited 14 years apart, in 1977 (earlier-born cohort) and 1991 (later-born cohort), Currin et al. reported that the later-born cohort were less rigid and stereotypical in their thinking about mental illness than the earlier-born cohort. Moreover, attitudes to mental illness appeared bound up with ageist misconceptions such that more individuals from the earlier-born cohort harboured stereotypes of ageing that included beliefs in the symptoms of mental illness as simply part of the ageing process. In contrast, the later-born cohort demonstrated a broader understanding of the range of issues related to mental illness, a greater awareness of services offered by mental health providers, and had used more mental health services in the past (suggesting a positive impact of personal experience). In addition, the later-born cohort were more educated, had higher incomes, better health, and were more likely to be open to treatment and willing to seek help for mental health difficulties. Cohort differences remained after controlling for various demographic factors including age and level of education. Based on these data, Currin et al. concluded that later-born older cohorts' biases toward mental illness are less negative than those of earlier-born older cohorts, ' $a$ finding with numerous implications for the design and implementation of mental health services for future cohorts of older persons' (p.506).

Thus, Currin et al. (1998) argue that the data are suggestive of an historical increase in the use of mental health services across older adult cohorts, an effect which may be obscured in general comparisons of younger and older adults. However, this study has been criticised on the grounds of positive sampling bias (participants in the study were well-educated and relatively healthy) and for confounding cohort and 
history, since the 1977 sample may have shown different attitudes upon follow-up in 1991 (Robb et al., 2003). Pepin (2006) also failed to find differences in attitudes between young-old and old-old cohorts in her study despite a trend for the old-old (i.e. earlier born) cohort to consider depression as normal, although again the sample was healthy, active and relatively homogeneous.

In a comparison of attitudes to mental health care in a community-dwelling sample of 474 older adults aged 65 and older and a nationwide sample of 1001 adults aged 21-65 in the U.S., Robb et al. (2003) set out to ascertain if differences in attitudes across age groups reflected familiarity with and experience of mental health issues. Although older people tended to have less personal experience and knowledge of mental health problems, they were also found to hold generally favourable attitudes towards mental health services. Attitudes of younger and older participants were similar in relation to increased likelihood of seeking treatment for severe mental disorders, perceived importance of mental health care, and concerns about cost and insurance coverage as barriers to care. Differences were found in relation to use of services (older participants were less likely to have used services), perceptions about less severe disorders (older participants viewed less severe conditions as simply part of life and prioritised self-sufficiency in handling such problems), referral sources (older participants were more likely than younger participants to consult their family doctor for mental health problems), and treatment preferences (older participants wanted access to the most highly trained doctoral level mental health professionals). In contrast to other studies (e.g. Lundervold \& Young, 1992), attitudes to mental 
health and mental health care, and concerns about the possible stigma associated with mental illness, did not appear to be overwhelming barriers to accessing services.

Finally, Davidson and Connery (2003) also found that older and younger adults held similar views in relation to depression, although positive attitudes were found to coexist with ageist and stigmatising attitudes in both groups. Thus in response to a vignette describing a depressed person portrayed as either aged 30 or aged 70 , both groups could identify the protagonist as experiencing problems that anyone could have, but were less likely to recognise depression if the protagonist was older. Older participants were also more likely to suggest that the protagonist (regardless of age) should 'pull himself together', but did not consider him to blame for his problems. In a recent follow-up study assessing differences in attitudes by age and gender, Connery and Davidson (2006) reported that older participants were less likely than younger participants to recognise symptoms of depression and more likely to hold less positive or more stigmatising attitudes about depression. However, when the protagonist in the vignette was described as an older person, participants from both younger and older age groups were less likely to recognise depressive symptoms than when the protagonist was described as a younger person, and were also less likely to report negative attitudes about depression (regardless of the protagonist's gender). Connery and Davidson (2006) suggest the latter finding indicates that participants were less harsh in their attitudes towards older adults with depressive symptoms. In general, males were less likely to recognise depressive symptoms than females, while females held more positive attitudes towards depression than males. 


\subsubsection{The Positivity Effect}

One possibility for the endorsement of more positive attitudes to mental illness in later life is provided by socioemotional selectivity theory, a central tenet of which is 'the positivity effect' (Carstensen et al., 1999, p.120), or the proposition of an increasing focus in later life towards positive or emotionally meaningful material in order to maintain emotional equilibrium. Socioemotional selectivity theory is described as 'a life-span theory of social motivation' (p.166) in which the perception of time or, more specifically, of endings, motivates older people (or individuals of any age facing premature endings), to selectively attend to positive information and to downplay negative information. In contrast, since time is perceived as open-ended earlier in life, younger adults may process negative information more thoroughly than positive information and lend greater weight to negative information in information processing (Carstensen \& Mikels, 2005). Of particular importance to the current research, Carstensen et al. (1999) speculate that the positivity effect may contribute to age differences in psychopathology, explaining the higher prevalence of depression and anxiety in the younger adult population. One could perhaps further speculate that the positivity effect might also be reflected in the endorsement of positive attitudes to mental illness by older people.

Socioemotional selectivity theory recognises that the emotional experience of older people is likely to be mixed given that both gains and losses typically accompany developmental change, but contends that the approach of endings directs the older person's attention to more emotionally meaningful experiences. Socioemotional selectivity theory therefore has intuitive appeal since it provides a partial explanation 
for the results of studies indicating the co-existence of negative and positive attitudes to mental illness in older people, although it has relatively little to say about the impact of ageist and age-related stereotypes on attitudes.

\subsubsection{Summary of Attitudinal Research Relating to Mental IIIness}

In summary, findings from the field of attitudinal research in relation to mental illness are somewhat mixed. On the one hand, several studies attest to the prevalence of stigmatising and negative attitudes towards mental illness, particularly mental illness as it occurs in later life (e.g. Crisp et al., 2005; Hayward \& Bright, 1997). Such attitudes are reported as apparent in both younger and older adults, although both inter- and intra-cohort attitudinal differences have also been identified (e.g. Brockington et al., 1993; Scottish Executive, 2004). On the other hand, other research indicates that attitudes are changing over time, becoming increasingly positive, and exhibit a greater degree of concordance between younger and older adults in this regard than previously thought (e.g. Currin et al., 1998; Segal et al., 2005). Many gaps in the literature are also apparent. For example, little is known about whether attitudes to mental illness, particularly in the oldest-old, are simply a reflection of broader underlying ageist stereotypes. Since stereotypes may be gradually reinforced over the life-span (Levy, 2003), this may account for the persistence of negative attitudes in this cohort despite the influence of the so-called positivity effect. 


\subsection{EXPERIENCE AND AWARENESS OF MENTAL HEALTH ISSUES}

More positive attitudes towards mental illness as a result of increased familiarity and personal experience of mental health difficulties have been reported in the literature. In relation to ageing, the latter effect may be underpinned by a general increase in opportunities to experience or come into contact with individuals with mental illness simply by virtue of living longer. In an early study, Lasoski and Thelen (1987) found that increased familiarity with psychological problems and services was associated with more positive age-related perceptions of psychopathology in middleaged people, suggesting that stereotypes might be influenced by education, knowledge and experience. Huxley (1993) found that those who had transient encounters with individuals with mental health difficulties tended to stigmatise them, while those who knew a mentally ill person who attended a treatment centre showed less stigmatising responses:

"it may be direct contact with people who receive helpful treatment for episodes of mental illness, rather than any mentally ill person encountered in the community, which destigmatises the subject of mental illness". (p.79)

Desforges et al. (1991) provide a useful examination of what has been termed the 'contact hypothesis', or the idea that contact with someone with mental illness predicts less prejudice and more positive behaviour. In a study of 95 students who engaged in structured co-operative contact with members of a disliked group (i.e. former mental patients), initially negative attitudes measured prior to contact were reported as more favourable following contact, with indications of generalisation towards the broader social group. Desforges et al. acknowledge, however, that attitudinal changes may not translate to changes in behaviour toward members of that group. 
There are also suggestions in the literature that people who experience a mental illness themselves may be less likely to endorse negative attitudes to mental illness. Lobban et al. (2003) suggest that the way in which the person views the experience is important in this regard. For example, many individuals with mental health problems seem to be able to avoid labelling themselves in a stigmatising way, and may evaluate themselves much more positively than they do 'the average mental patient' (Hayward \& Bright, 1997, p.349). Conversely, the double-stigmatisation hypothesis (Sartorius, 2003) suggests that experiences of mental illness are likely to be particularly damaging for the older individual since this will bring them into direct proximity of negative societal attitudes relating to age and mental illness, as outlined below.

\subsection{ATTITUDES TO AGEING}

\subsubsection{Societal and Structural Ageism}

The term 'ageism' was originally coined by Butler (1969) to refer to:

" $a$ deep-seated uneasiness on the part of the young and middle-aged - a personal revulsion to and distaste for growing old, disease, disability; and fear of powerlessness, uselessness, and death". (p.243)

Although ageism is now more broadly defined to include discrimination on the basis of age at any point in the lifespan, the original sense of devaluation of older people remains (Knight, 1999). Somewhat disturbing is the ease with which this form of discrimination appears to be acceptable, almost commonplace, compared with other types of prejudice in society, and does not appear to invoke the same sense of shame 
(Levy \& Banaji, 2002). For example, Laidlaw et al. (2003) discuss the potentially corrosive nature of several pervasive myths and stereotypes of ageing which perpetuate a pessimistic view of growing older, such as: 'you can't teach an old dog new tricks', 'it must be terrible getting old', and 'to be old is to be sick' (pp.21-24). Structural ageism has also been proposed to underlie the provision and delivery of health care services, including mental health services, for the elderly (Bowling, 1999; Levy \& Banaji, 2002). Despite reports of some steady (albeit marginal) improvements in public attitudes towards older people (Palmore, 2004), 'pockets of negativism toward the aged still exist occurring subtly, covertly and even unconsciously' (Butler, 1993, p.76).

\subsubsection{Negative Stereotypes of Ageing and Internalised Ageism}

The prevailing social stereotype of ageing in Western societies is predominantly negative (Levy, 2003). Two meta-analyses summarising attitudes about older people have shown that in Western cultures older adults are generally rated more negatively than younger adults (Kite \& Johnson, 1988; Kite et al., 2005), however recent research has also indicated the existence of similar negative stereotypes in Eastern cultures (e.g. China, Thailand, and the Phillippines; Harwood et al., 2001), although some degree of multidimensionality (i.e. co-existence of both positive and negative traits) has also been proposed (Boduroglu et al., 2006). Generally, studies of ageing stereotypes suggest that both older people and younger people share a generally negative conception of old age, although findings vary according to study methodology. Thus, studies of explicit attitudes tend to report that older people 
judge their own age group more positively than do young people (demonstrating ' $i n$ group bias'; Hummert et al., 2002, p.484) even though both age groups hold generally negative attitudes about old age (e.g. Kite et al., 1991), although this has not been consistently found (Hummert et al., 2002). Conversely, studies of implicit attitudes indicate that older people tend to hold more negative attitudes towards their own age group than do the young, demonstrating 'out-group favouritism' (e.g. Levy \& Banaji, 2002, p.52). One potential explanation for these conflicting results may be the influence of cultural norms or self-presentational influences on reporting of explicit attitudes (such that people do not wish to appear biased or prejudiced), which studies of implicit attitudes are proposed to circumvent.

Studies assessing the nature of implicit attitudes typically utilise priming methodologies (i.e. measuring implicit age stereotypes through activation of preexisting schemas) or implicit association tasks (IAT). IAT involves recording of response latencies to category judgement tasks, and is based on the logic that judgements congruent with participants' implicit associations of a target category (e.g. pairing the target category of 'old' with an evaluative dimension such as 'frail'), will be easier and thus made more quickly than those that are incongruent (Hummert et al., 2002). Using IAT methodology, Levy (2003) reports results from a series of studies indicating that members of all social groups tested, other than the aged, generally show more positive implicit attitudes towards their own group compared to non-group members. Levy (2003) also suggests that implicit age attitudes can operate independently of explicit attitudes, with the latter showing less negativity overall. However, these results are not without criticism. For example, 
pertinent critiques of the validity of IAT methods in older people have highlighted potential confounding effects of age-related cognitive slowing in analysis and interpretation of IAT measures in this population (Hummert et al., 2002). That is, IAT results may reflect not only implicit perceptions but also age group differences in speed of processing (although one might also expect that this would demonstrate a consistent effect across tasks that could be controlled for).

Notwithstanding such critiques, Levy (2003) presents persuasive evidence that negative implicit ageist attitudes and stereotypes operate unconsciously to impact upon judgements, perceptions, beliefs and behaviours, and are consolidated over time. Specifically, Levy (2003) puts forward a developmental hypothesis which posits that as people grow older, ageist stereotypes internalised at a young age gradually become self-stereotypes, thus influencing the older person's perception of other older individuals as well as of themselves. The impact of negative selfstereotypes on performance and behaviour has been demonstrated in a series of studies of stereotype priming, in which participants are first 'primed' with traits of positive (e.g. 'wise') or negative (e.g. 'senile') old-age stereotypes prior to undertaking a performance task. Levy (1996), and Levy and Banaji (2002), report negative effects of self-stereotyping on memory, handwriting, walking, and expressed will to live of older participants in comparison to younger participants, although some of this work suffers from general confounding of motivation and ageing, psychomotor slowing, and lack of appropriate control measures. 
If older people do indeed harbour implicit negative self-stereotypes of ageing, this may lead to harmful and insidious effects. In relation to onset of mental health difficulties, for example, the older person may be far less likely to recognise the origin of any symptoms they might experience (Levy \& Banaji, 2002). Support for this hypothesis is forthcoming from Law (2003) who demonstrated that the understandability phenomenon in older people was reflective of an ageist misconception. Taking this point further, it does not seem unreasonable to suggest that the attitudes older people hold in general in relation to mental illness may be a function of this same mechanism.

\subsubsection{Positive Stereotypes and Successful Ageing}

Despite a general focus within the empirical literature on the existence and prevalence of negative ageing stereotypes, it is important to draw attention to research suggesting that most individuals are actually aware of both positive and negative stereotypes (Harwood et al., 2001). There are indications, for instance, that many people designated as elderly by wider society do not in fact self-identify as old (Degnen, 2007), creating a disjuncture between lived experiences of ageing and the prevailing negative and limiting stereotypes of old age. In addition, there is evidence to suggest that positive ageing stereotypes are also internalised and can be cultivated over time, with those holding positive stereotypes prior to old age more likely to carry these forward into later life and to benefit as a result (Levy, 2003). Unfortunately, very little research has examined older peoples' perceptions of the 
ageing process. Two notable exceptions can be found in the work of Levy et al., (2002) and Laidlaw et al. (2007).

In the longitudinal study by Levy et al., (2002), positive self-perceptions of ageing were found to impact on longevity in a reasonably large sample of 660 older people, with the median survival of those holding positive self-perceptions 7.5 years longer than those holding negative self-perceptions, a finding maintained after controlling for age, gender, socio-economic status and health. In the study by Laidlaw et al. (2007), which describes the development of a cross-cultural measure of attitudes to ageing (the attitudes to ageing questionnaire or 'AAQ'), those who age most successfully are proposed to hold positive perceptions of ageing in which growth and development are perceived as still possible despite the psychosocial losses and physical challenges associated with ageing.

The concept of successful ageing (e.g. Baltes \& Smith, 2002; Rowe \& Kahn, 1998; Vaillant, 2002) runs counter to the 'popular' negative ageing stereotype and selfstereotype of physical and psychological decline. Models such as selective optimisation with compensation (Baltes \& Smith, 2002) discussed earlier (see Section 1.4.1.1), and the decline, change and development model of successful ageing (Vaillant \& Mukamal, 2001) emphasise not only physical attributes of ageing, but also social and emotional maturation over the life course. In Vaillant's work from the Harvard study of adult development, in which he carried out a longitudinal follow-up study of two male cohorts (237 college students, and 332 core-city youth), ageing well was associated more with various aspects of psychological health (such 
as maintaining intimacy, growth, participation and a sense of meaning in one's life) than with physical factors. Furthermore, Vaillant argues that, while one cannot ignore the physical declines associated with growing older, ageing should be viewed principally as a time of change rather than decline, and of development through maturation, increasing wisdom, tolerance and experience. However, despite the obvious importance of this work, the study is hampered by sampling limitations (e.g. reported data are drawn from a sample of white American men born in the 1920s) and missing data (e.g. length of active life data for the youngest men), thus precluding firm conclusions.

In sum, positive stereotypes and models of successful ageing challenge the traditional pessimistic view of ageing found in western, and, more recently, in eastern cultures. However, there is little in the way of empirical research investigating the relative strength of and relationship between positive and negative stereotypes of ageing, broader social cognitions (e.g. attitudes, perceptions, beliefs), and behaviour.

\subsection{SUMMARY OF MAIN RESEARCH FINDINGS}

In summary, the relationship between attitudes to mental illness and attitudes to ageing is likely to be complex and may be mediated in some way by personal experience. However, in general, these factors are poorly understood in both younger and older adult populations. In the empirical literature single dimensions are often addressed in isolation although this is unlikely to enhance knowledge since 
patterns of interaction between variables are likely to occur. Research is therefore required which specifically addresses the interplay between different factors impacting the attitudes of older people with and without mental health problems. A greater awareness of factors influencing older peoples' attitudes to mental illness may improve recognition, presentation, and treatment of mental disorders in this population, and so enhance individual well-being and quality of life. It may also inform the development of integrated public policies regarding mental health promotion, illness prevention, service provision and development, and help to counter ageist and stigmatising societal attitudes through improved awareness and education.

The studies outlined in this chapter highlight the complexity of issues to be considered when exploring attitudes to mental health and mental illness in the older adult population. There is some evidence to support the notion that older people hold generally negative views of mental illness, although it is unclear whether such views are changing (i.e. becoming increasingly positive) with successive cohorts, or are qualitatively different from those held by younger people since many studies lack a younger adult comparison group. In addition, assumptions are frequently made regarding the views of older people in the absence of direct assessment, and many studies suffer from methodological limitations such as sampling bias. For example, a large proportion of studies report the attitudes of healthy individuals, while the opinions of clinical samples have largely been neglected. Furthermore, cohort differences such as those found between young and old, and between young-old and 
old-old cohorts, may be an artefact of cross-sectional study design, while longitudinal studies have not been undertaken.

Current evidence also fails to address several pertinent questions. For example, if older adults do indeed hold negative perceptions of mental illness, to what extent do such views hold in relation to age, experience, and service use? Moreover is it possible that older peoples' attitudes to mental illness in later life are a reflection of more fundamental internalised and implicitly negative views of ageing? The current study aims to explore these issues further in a comparison of 'healthy' older people (i.e. those not attending mental health services) and mental health service users through a combination of focus groups and self-report questionnaires.

\subsection{RESEARCH AIMS AND HYPOTHESES}

\subsubsection{Aims}

Older people are often assumed to have negative attitudes towards mental illness and mental health services which impede recognition, presentation and help-seeking behaviour, and inevitably impact on well-being. However, there lacks a consistent empirical body of evidence to support the validity of these assumptions, and a dearth of studies assessing the correlates, if any, of older people's attitudes. Therefore, the main aim of this study was to replicate and extend previous research by first exploring and describing the attitudes of older people to mental illness. A further aim was to explore correlates of attitudes by considering whether attitudes were related to personal experience and/or ageist misconceptions, and to compare the 
views of 'healthy' older people and service users. Finally, the association, if any, between attitudes and well-being, and between attitudes and health-related behaviours was also considered.

\subsubsection{Hypotheses}

1.9.2.1 Hypothesis 1: Older people will endorse more negative attitudes to mental illness than younger people. Intra-cohort attitudes will differ by age and gender.

Many studies suggest that older people have negative attitudes towards mental illness, and are inherently more negative in their attitudes than younger adults (e.g. Lundervold \& Young, 1992; Scottish Executive, 2004). It is therefore hypothesised that in comparison to younger adults, older people will hold more negative views about mental illness. Within the older adult sample, cohort and gender differences are also proposed to exist, such that it is anticipated that old-old participants (i.e. those aged 80 plus) will endorse more negative views than young-old participants (i.e. those aged 65-79), and older males will hold more stigmatising attitudes towards mental illness than older females.

1.9.2.2 Hypothesis 2: Participants currently in receipt of mental health services will endorse more positive attitudes to mental illness than those not in receipt of services. Those with prior experience of mental illness/mental health services will endorse more positive attitudes than those without such experience.

Several studies have reported on the beneficial effects of personal experience of mental health problems and use of services on attitudes (e.g. Scottish Executive, 2004). It is therefore hypothesised that attitudes to mental illness in later life will be related to personal experience. Thus it is anticipated that participants currently in 
receipt of mental health services will endorse more positive views in relation to mental illness in later life than participants not attending services. Furthermore, those with prior experience of mental illness or mental health services, will endorse more positive attitudes than those without such experience.

1.9.2.3 Hypothesis 3: Negative attitudes to ageing will be associated with negative attitudes to mental illness in later life. Attitudes to ageing will vary significantly by clinical status and age (i.e. clinical participants and the oldest-old will endorse more negative attitudes).

In accordance with several theoretical perspectives (e.g. Levy, 2003; Sartorius, 2003), it is anticipated that negative attitudes to ageing will be associated with negative attitudes to mental illness in later life. A secondary hypothesis proposes that clinical participants will endorse more negative attitudes to ageing than nonclinical participants, and that the oldest old will endorse more negative attitudes than the young-old.

\subsubsection{Hypothesis 4: Reported engagement in health-related behaviours and subjective well-being will be lower in the clinical sample. Negative attitudes to ageing and to mental illness will be associated with lower endorsement of health-related behaviours and poorer subjective well-being across the entire sample.}

Older adults are a growing sector of the population and it is therefore important to examine the implications of negative attitudes on well-being and behaviour. It is hypothesised that the clinical group will report less engagement in health-related behaviours and lower subjective well-being than the non-clinical group. It is further hypothesised that negative attitudes to ageing and to mental illness will be associated with fewer reported health behaviours and poorer subjective well-being across the entire sample. 


\section{2 - METHOD}

\subsection{DESIGN}

The study combined qualitative and quantitative methods. Initially, focus groups were undertaken with clinical and non-clinical participants with the primary aim of piloting a draft questionnaire, and to provide a descriptive overview of themes describing older peoples' attitudes to mental illness. A cross-sectional independent groups design was then employed to compare attitudes to mental illness in a clinical sample of older people currently in receipt of mental health services (psychological or psychiatric services), to attitudes of a sample of older people from the general (i.e. non-clinical) population. All participants were requested to provide sociodemographic information in the form of a brief questionnaire, and additionally completed a study questionnaire assessing the primary variables of interest including attitudes to mental illness, attitudes to ageing, general health and well-being, and engagement in health behaviours. Relationships between variables were assessed using multivariate and correlational analyses.

\subsubsection{Ethical Approval}

Ethical approval was sought and obtained from the local Research Ethics Committee, and permission was sought and received from the Research and Development department of the primary care division of the Community Health Partnership (CHP) to recruit participants for both the clinical and non-clinical samples (Appendices 1.1 
and $1.2^{4}$ ). During the course of the study the researcher was also granted access to an additional non-clinical sample of participants via an older people's forum based in another locality. Permission was therefore sought and obtained from the local research ethics committee to extend the research, and from the forum to undertake questionnaire completion with their membership.

\subsection{PARTICIPANTS}

\subsubsection{Inclusion and Exclusion Criteria}

Participants were recruited to the study according to the following inclusion criteria. For the non-clinical sample, participants had to be age 65 and above, communitydwelling, not currently in receipt of mental health services, and able to provide written informed consent. For the clinical sample, the same criteria applied although participants had to be currently in receipt of mental health services. In both conditions, participants were excluded from the study if they were under 65 years (age 65 was selected as the standard service cut-off criterion for access to older people's mental health services in the locality where the research took place), if there was evidence of organic deterioration or significant cognitive impairment, if the participant was currently in receipt of electroconvulsive therapy, if there were indications of poorly managed severe and enduring mental illness, if there was evidence of suicidality, substance abuse, or previous head injury (all of the latter to be ascertained via clinician judgement for the clinical sample, or group

\footnotetext{
${ }^{4}$ Note that to protect confidentiality, all identifying information has been removed from study correspondence.
} 
representative judgement for the non-clinical sample). The source and method of recruitment for both samples is described below.

\subsubsection{Non-clinical Sample}

A non-clinical sample of participants was identified with the assistance of the local branch of Age Concern (AC) and of the Elderly Forum (EF), with additional participants identified latterly through another Older People's Forum (OPF) in a different locality. The researcher met with representatives from all groups to first discuss the aims and objectives of the study, to answer questions, and to request assistance with study recruitment. Representatives from each of the various organisations were then requested to identify and inform potential participants about the study. Subsequently, the researcher made a series of presentations about the study directly to groups of potential participants, and study invitation packs (containing a study invitation letter and information sheet; Appendix 2) were distributed by group representatives as appropriate.

Approximately $521^{5}$ packs were distributed to Age Concern and the Forums (between November 2006 and June 2007) for circulation to prospective participants. Of these, 87 requests were received from individuals willing to take part in the research (a response rate of 16.7 per cent), of whom 50 (15 men, 35 women; mean age 79.84 years, SD 8.05; age range 65 - 99) completed and returned questionnaires

\footnotetext{
${ }^{5}$ Note that although group representatives were requested to maintain records of the number of packs distributed, packs were most commonly cascaded through smaller member groups who failed to record numbers. It is therefore unclear how many of the 521 packs were actually circulated to prospective eligible participants.
} 
(i.e. 57.5 per cent). Due to the indirect and phased method of study recruitment (and to protect individual anonymity) it was not possible to obtain additional data for nonrespondents or ineligible participants, thus precluding a demographic comparison with respondents.

\subsubsection{Clinical Sample}

Patients currently in receipt of treatment for mental health difficulties such as anxiety or depression, and meeting study inclusion criteria, were identified with the assistance of clinicians from the Area Clinical Psychology Service and the Area Old Age Psychiatry Service. The researcher initially met with clinical staff from both services, as well as staff from two local geriatric day hospitals (included in the Old Age Psychiatry Service), to describe the purpose and design of the study and to address any questions. Those clinicians/psychiatric nursing staff agreeable to assist with recruitment were requested to identify and inform suitable patients about the research study and to provide them with a study invitation pack (Appendix 2).

Approximately $188^{6}$ packs were distributed to individual clinicians and psychiatric nursing staff (between November 2006 and June 2007) for circulation to prospective participants. Of these, 34 requests were received from individuals willing to take part in the study (a response rate of 18.1 per cent), of whom 24 ( 9 men, 15 women; mean age 72.59, SD 5.42; age range 65-83) completed and returned questionnaires (i.e. 70.5 per cent). No requests were received from patients currently being seen by

\footnotetext{
${ }^{6}$ Note that although clinical staff were requested to maintain records of the number of packs individually distributed to patients, many forgot to do so, and therefore it is unclear how many of the 188 packs were actually circulated to prospective eligible participants.
} 
psychiatrists (possible reasons for this are considered in Section 4.4.2.2). As for the non-clinical sample, no additional data were obtained for non-respondents or ineligible participants.

\subsubsection{Total Sample}

Overall, approximately 709 study invitation packs were distributed, and 121 questionnaires were requested from potential participants meeting study inclusion criteria (an overall response rate of 17.1 per cent). Of these, 74 individuals completed and returned questionnaires (61.2 per cent). Details of sample recruitment across clinical and non-clinical populations are provided in Table 3 below. The final sample was comprised of 50 females (67.6\%) and 24 males (32.4\%). The overall mean age of the participants was 77.63 years, SD 8.04.

Table 3: Breakdown of Sample and Participation Rates by Source

\begin{tabular}{|c|c|c|c|c|c|c|c|c|}
\hline & \multicolumn{3}{|c|}{$\begin{array}{c}\text { Non-Clinical } \\
\text { Sample }\end{array}$} & \multirow[b]{2}{*}{ Total } & \multicolumn{3}{|c|}{ Clinical Sample } & \multirow[b]{2}{*}{ Total } \\
\hline & AC & $\mathbf{E F}$ & OPF & & Psychol. & Psyt. & $\begin{array}{c}\text { Day } \\
\text { Hospital }\end{array}$ & \\
\hline $\begin{array}{l}\text { Number packs } \\
\text { distributed* }\end{array}$ & 205 & 272 & 44 & 521 & 70 & 86 & 32 & 188 \\
\hline $\begin{array}{l}\text { Number Q's } \\
\text { requested }\end{array}$ & 30 & 22 & 35 & 87 & 18 & 0 & 16 & 34 \\
\hline $\begin{array}{l}\text { Number } \\
\text { participating }\end{array}$ & 21 & 20 & 9 & 50 & 9 & 0 & 15 & 24 \\
\hline $\begin{array}{l}\text { Number not } \\
\text { participating** }\end{array}$ & 9 & 2 & 26 & 37 & 9 & 0 & 1 & 10 \\
\hline
\end{tabular}

* Estimated (see text for description).

** Reasons for non-participation not known (one non-clinical participant excluded for not meeting age criteria). 


\subsection{MEASURES}

\subsubsection{Demographic information}

Demographic information was sought on age, gender, marital status, educational attainment, occupation, and living arrangements, as well as objective and subjective health status, and previous (individual and family) history of attending mental health services for mental health difficulties in order to assess prior experience (Appendix 2.4). Details of sample demographics are provided in Section 3.2.1.

\subsubsection{Questionnaire Measures}

In addition to provision of sociodemographic information, responses were sought to the following items/questionnaires (Appendix 2.4) as follows:

- Attitudes to Mental Illness:

- 9 items from the National Survey (Scottish Executive, 2004);

- Two subscales ('Ageism' and 'Stigma') from the Barriers to Mental Health Services Scale (BMHSS; Pepin, 2006);

- Understandability Questionnaire (Law, 2003).

- Attitudes to Ageing:

○ Attitudes to Ageing Questionnaire (AAQ; Laidlaw et al. 2007);

○ The Rame Questionnaire (Parnell et al., 2001).

- Health and Well-being:

○ The General Health Questionnaire (GHQ; Goldberg, 1992).

- The Reported Health Behaviours Checklist (RHBC; Prohaska et al., 1985). 


\subsubsection{Attitudes to Mental IIIness}

\subsection{Items from the National Survey (Scottish Executive, 2004)}

Nine items were included (with permission) from the second National Scottish Survey of public attitudes to mental health ('Well? What do you think?', Scottish Executive, 2004). The items reflect prevalent themes identified in the literature regarding public perceptions of mental illness, and were constructed by a multidisciplinary advisory group to facilitate comparison with similar surveys undertaken in England (DOH, 2003) and Ireland (The Mental Health Ireland study; Millward Brown, 2003). Responses are scored on a Likert scale ranging from $1=$ strongly agree to 5=strongly disagree, with $6=$ don't know. At the time of writing no details were available regarding the psychometric properties of the items.

\subsection{Barriers to Mental Health Services Scale (BMHSS subscales; Pepin, 2006)}

The BMHSS was recently developed by Pepin (2006) to measure barriers to mental health services in younger and older adults. The BMHSS comprises a total of 10 subscales (58 items) representing both internal and external barriers to help-seeking. Data reported by Pepin (2006) regarding internal consistency of the BMHSS indicate sub-scale alphas range from 0.32 to 0.78 , with 5 of the 10 sub-scale alphas scoring over 0.70. Subscales can be considered independently, and those relating to 'Ageism' (6 items; range 6-30) and 'Stigma' (5 items; range 5-25) were considered most relevant for inclusion in the current study (Cronbach's alpha 0.63 and 0.73 , respectively). Both sub-scales provide an indication of the impact of negative 
attitudes on help-seeking behaviour (e.g. Item 4, Ageism subscale - 'I would not see a psychologist because psychologists think working with someone my age is a waste of time'). Minor modifications to the wording of items were made in order to increase item clarity for a U.K. population (e.g. replacement of the term 'psychotherapist' with 'psychologist'). Responses are scored on a five-point Likert scale where $1=$ strongly disagree, and 5=strongly agree. Subscale scores are derived from summing individual item scores. Positively oriented items on both subscales are reverse coded such that higher scores are indicative of more negative attitudes to mental illness.

\subsection{Understandability Questionnaire (Law, 2003)}

The Understandability measure comprises three attitudinal statements to depression in later life, designed to reflect the following conceptualisation: "It is natural to be depressed in late life because there are good reasons for it, therefore, there is no point in treatment" (Law, 2003, p36). The statements were generated from the relevant literature and from the results of focus group discussions and clinical experience with older people (see Law, 2003, for further details). Items are rated on

a seven-point Likert-type scale ranging from 1=agree strongly to $7=$ disagree strongly. Items are reverse coded and summated to obtain a total 'understandability' score ranging from 3 to 21 , with higher scores indicative of greater belief that depression is a normal consequence of old age. Two additional items are included in the scale to assess for intergenerational bias (i.e. the perception that younger people have no justification for feeling depressed; otherwise referred to as the 
understandability phenomenon in reverse, or the idea that depression is only 'understandable' in older people), although these latter items are not intended to yield a composite score.

\subsubsection{Attitudes to Ageing}

\subsection{Attitudes to Ageing Questionnaire (AAQ) (Laidlaw et al., 2007)}

The AAQ (Laidlaw et al., 2007) is a 24-item self-report measure designed to assess attitudes towards ageing and the ageing process. The measure was developed within the context of a multi-centre collaborative research study (Power et al., 2005) in which a total of 5566 participants (age range 60-99) from 20 centres worldwide contributed to scale development. The AAQ comprises three subscales relating to 'psychological growth' (explicitly positive focus; 8 items), 'psychosocial loss' (explicitly negative focus; 8 items), and 'physical change' (mixed physical functioning focus; 8 items), with good psychometric performance indicated through both classical (e.g. exploratory factor analyses) and modern methods (e.g. confirmatory factor analyses; item response theory). Published Cronbach alpha values for each of the subscales range from 0.68 to 0.84 , with an overall scale fit of 93.8 per cent for the three-factor model. Recommended scoring of the scale consists of a profile set of three subscale scores. Items relating to each subscale are scored on a five-point Likert scale with variation in response descriptors across items, as follows: items 1-7, 1=strongly disagree to 5=strongly agree; items 8-24, 1=not at all true to $5=$ extremely true. Items are summed to provide an overall score for each subscale (range 8-40), with a higher score reflecting stronger endorsement of the 
predominant theme of that particular subscale (e.g. higher score on "psychological loss' = stronger endorsement of loss-deficit model of ageing). An overall total score can also be computed (supported by the existence of a higher order factor in the confirmatory factor analyses) by summing all 24 items in the scale (range 24-120) following reversal of positively oriented items, such that a higher score is indicative of more negative attitudes to ageing.

\subsection{The Rame Questionnaire (Parnell et al., 2001)}

The Rame questionnaire (Parnell et al., 2001) is designed to measure internalised ageism in an older adult population. The measure comprises 23 items relating to fear of the ageing process, deterioration in physical and cognitive ability, illness, inability to learn new tasks, reduction of sexual desire, and beliefs that older people require constant help and contribute little to society. The scale was developed in a sample of 40 older people attending day centres across the UK, and is reported to demonstrate good internal reliability $($ Cronbach's alpha $=0.81)$. However, to date, the scale has been insufficiently tested to determine broader psychometric properties such as factor structure, or the establishment of normative scores (Law, 2003). The Rame contains 8 positively oriented items (e.g. 'I am keeping in touch with today's society') and 12 negatively oriented items (e.g. 'My best achievements are in the past'), in addition to three re-phrased repeat items included to assess respondent understanding (not scored). Items are scored on a four-point Likert scale ranging from 'disagree strongly' to 'agree strongly', and scored either 0 to 3 or 3 to 0 , as appropriate, with 3 indicating higher internalised ageism (i.e. positively oriented 
items are reversed scored). Items are summed to provide a total scale score out of 60.

\subsubsection{Health and Well-being}

\subsection{The General Health Questionnaire (GHQ-12; Goldberg, 1992)}

The General Health Questionnaire (GHQ; Goldberg, 1978) is a widely used and validated measure of non-psychotic psychiatric symptoms in the general population. It has been included as a measure of possible psychiatric morbidity in a number of attitudinal studies (e.g. the National Survey - Scottish Executive, 2004; Health Survey for England - Department of Health, 2002; The Scottish Health Survey Scottish Executive, 2005) including both younger (i.e. 16-64) and older adults (i.e. 65 plus). The 12-item version of the General Health Questionnaire (GHQ-12; Goldberg, 1992) was used in the current study as a shortened version of the wellvalidated full version, the GHQ-60, as it has equally good psychometric properties (i.e. in a series of studies Cronbach's alpha ranged from 0.82 to 0.90 ; split-half reliability was 0.83 , and test-retest reliability was 0.73 ; sensitivity in detecting cases of psychiatric disorder reported as $93.5 \%$, with $78.5 \%$ specificity; see Johnston et al. 1995 for a brief review).

The GHQ-12 has been criticised in the literature for being primarily a measure of negative well-being since it is typically concerned with the presence or absence of psychiatric symptoms (Huppert \& Whittington, 2003). Positive well-being, in contrast, is defined not merely as the absence of symptoms but rather by positive endorsement of positively worded items relating to, for example, personal growth, 
autonomy and self-acceptance (see Parkinson, 2006, for discussion of an alternative measure). However, the critique of the GHQ-12 as a measure of negative well-being may be relevant only in so far as the traditional case ascertainment scoring method is used. In contrast, scoring the GHQ-12 by the lesser-used Likert scaling method allows one to obtain a range of responses across both positively and negatively oriented items such that it is possible to capture something more than simply the absence of symptoms. For example, in response to the item 'have you recently been feeling unhappy and depressed', respondents may answer in the negative 'not at all' or 'no more than usual' (thus indicating absence of symptoms but not necessarily positive well-being), yet may later also endorse a positive response of 'more so than usual' to the item 'have you recently been feeling reasonably happy' (reflective of positive subjective well-being). The Likert scoring method was used in the current study since it also reportedly gives a less skewed distribution of scores (Johnston et al., 1995). Item responses are assigned scores of $0,1,2$, or 3, ranging from 'Not at all' to 'Much more than usual', as appropriate. Responses are then summed to provide an overall total score (from 0-36) with a higher score indicating poorer psychological health. Cut-off thresholds have not been validated for Likert scoring although higher cut-offs may be necessary for respondents with somatic symptoms which can inflate scores, and may be of particular relevance for older people suffering from depression or anxiety (Johnston et al., 1995).

\subsection{Reported Health Behaviours Checklist (RHBC; Prohaska et al., 1985)}

The RHBC (Prohaska et al., 1985) is a 21-item self-report measure of respondent 
health-related behaviours. The first 15 items of the scale relate to preventative health actions such as taking vitamins, undertaking regular aerobic exercise, and having regular medical check-ups. A further six cognitive/affective items cover aspects such as avoidance of emotional distress, positive thinking, and acceptance. Items are scored on a five-point Likert scale where response options reflect frequency of performance of each behaviour ranging from $1=$ never to $5=$ always. The total score for the scale, and totals for the 'Heath Action' (range 15-75) and 'CognitiveAffective' (range 6-30) sub-scales can be calculated (Kincey et al., 2003). High scores indicate high levels of reported positive health behaviours. No data on scale internal consistency are available as Prohaska et al. considered items individually, and no subsequent published papers could be located reporting psychometric properties of the RHBC or its subscales. In the current study, prior to multivariate analysis, scale and sub-scale reliability will therefore be explored.

The main value of the RHBC lies in the availability of normative data for three age groups spanning age 20 to age 60 and over. Results of age comparisons show that older people generally report higher frequencies of health-promoting actions and lower frequencies of risk-inducing actions (Prohaska et al., 1985), although to the author's knowledge responses to the scale have not yet been compared in clinical and non-clinical samples of older people, nor has the scale been assessed in relation to broader attitudinal variables. While the RHBC may provide a useful yardstick or measure of self-reported health-related behaviours, it is important to note that, like other measures of this type, the RHBC is limited by a paucity of evidence of validation against actual behaviours. 


\subsection{FOCUS GROUP PROCEDURE}

\subsubsection{Description}

Focus groups were undertaken with clinical and non-clinical participants as outlined in Section 2.1. A brief rationale for use of focus groups in social science research is provided in Appendix 3.1.

Prospective participants were approached through a variety of routes (e.g. through local pre-existing Age Concern groups for the non-clinical sample, or by clinical colleagues for the clinical sample). Participants were provided with a copy of the focus group information sheet (Appendix 3.2), and, if agreeable to participation, were subsequently asked to complete an informed consent form prior to the group (Appendix 3.3). Each focus group ran for two hours, with a break mid-way for refreshments. The structure of group sessions was designed to minimise bias in participant responses. Thus, a general discussion of participant attitudes was undertaken prior to piloting study documents. Participants were not required to complete the questionnaire, but rather were requested to read through it and provide feedback in relation to length, aesthetics (e.g. colour, design and layout), wording, structure, readability, and overall content. Two moderators (i.e. the study researcher and clinical supervisor) co-facilitated each session utilising guidelines to enhance consistency of approach in line with recommendations by Wilkinson (2003) (Appendix 3.4). Discussions were audio-taped and transcribed in preparation for qualitative analysis (Appendix 3.5). Transcriptions were supplemented by moderator observations of focus group processes (e.g. perceived atmosphere of the group etc.). 


\subsubsection{Focus Group Composition}

In line with recommendations in the literature relating to an optimal focus group size of between six and eight participants (e.g. Wilkinson, 2003), the non-clinical focus group comprised six women ${ }^{7}$ (mean age 82.3 years, SD 3.01), all members of Age Concern User Group Panels ${ }^{8}$. As this was a pre-formed, relatively homogeneous group, it was possible to observe naturally occurring interaction between participants already known to one another (Kitzinger, 1995). The focus group was held during one of the group's regular meetings, with those wishing to participate attending. Moderators perceived the general atmosphere of the group as relaxed and informal, and conducive to discussion.

The clinical focus group sample comprised six participants (four women and two men; mean age 76.2 years, SD 3.43) currently attending a local psycho-geriatric day hospital primarily for problems of depression and/or anxiety (i.e. participants shared a history of mental health difficulties). Moderators perceived the atmosphere of the group as initially apprehensive although the group later settled into an open discussion.

Additional focus group participant characteristics are outlined in Appendix 3.6. Ethical issues pertaining to focus groups are discussed in Appendix 3.9.

\footnotetext{
${ }^{7}$ One prospective male participant declined to take part.

${ }^{8}$ User Panels are an Age Concern Scotland initiative which provide older adults with the opportunity to discuss topics of mutual interest and share personal experiences of growing older. Members must meet the inclusion criteria of being aged over 70 and living independently.
} 


\subsubsection{Modification of Study Materials}

Focus group participants were presented with initial drafts of all study documents. Two versions of the questionnaire were circulated (containing the same content but with differing designs), presented on buff coloured paper to enhance visual acuity. In general, participants from both groups considered that the questionnaire and study documents were well-designed with adequate font size and good spacing, clear instructions, and were easy to follow. Two participants from the non-clinical group commented on the length of the questionnaire, indicating that they would be inclined to complete it in stages rather than at one sitting, although they were not unduly concerned about length ("we have lots of time to do it"). All participants agreed that they would be much more likely to complete the questionnaire if they had been approached to take part in the study in advance of the questionnaire being sent to them. No content gaps were identified in the questionnaire and no suggestions were made for inclusion of additional items. A few minor changes were proposed to improve item wording (summarised in Appendix 3.7) prior to printing and distribution. No modifications were made to study letters or information sheets.

\subsection{MAIN STUDY PROCEDURE}

\subsubsection{Pilot Test}

A pilot-test was undertaken to ascertain an approximate questionnaire completion time given that questionnaire length was raised in the focus group discussions as a potential obstacle to study participation (see above). Two women and one man (mean age 70.5 years, SD 0.71) identified through Age Concern, who did not wish to 
take part in the main study but who otherwise met inclusion criteria for the nonclinical sample (see Section 2.2.1), were timed whilst completing the questionnaire. Average completion time was 30.5 minutes. An indication of average time to complete the questionnaire of between 30 to 40 minutes was therefore included on the participant information sheet.

\subsubsection{Non-Clinical Sample}

Following an initial invitation to take part in the study by local group representatives, individuals agreeable to participation were requested to return the reply-slip contained within the invitation pack directly to the researcher or to indicate their agreement via the Age Concern or Forum representative, as appropriate. Participants were subsequently sent or provided with a copy of the study questionnaire for completion together with covering letter, and reply-paid envelope for direct return (Appendix 2). Alternatively, respondents could opt to return their questionnaire to the group representative for forwarding to the study researcher, if preferred. The researcher was also available to meet with respondents to discuss the study and assist with questionnaire completion as required (e.g. if participants were visually impaired). Questionnaires were id-linked, and participant contact information, if provided, was held separately from questionnaire response data. In accordance with Ethical Committee recommendations, study consent was considered implicit through questionnaire completion and postal return. Otherwise (i.e. in the case of non-postal questionnaire distribution and collection), participants were requested to complete an informed consent form. Follow-up reminders to potential participants were not sent 
in accordance with Ethical Committee requirements regarding use of identifying information (see Appendix 1.3).

\subsubsection{Clinical Sample}

Further to initial invitation to take part in the research by clinical staff, those individuals agreeable to participation were then requested to return the reply-slip contained within the invitation pack directly to the researcher or to indicate their agreement via the clinician/clinical staff member. In all other respects, the procedure for recruitment of the clinical sample was the same as that outlined for the nonclinical sample. Participants could opt to return their questionnaires by post or to the clinician/clinical staff member, as preferred.

\subsection{STATISTICAL POWER}

Sample size was estimated for the current study for both the between-subjects and regression analyses. A prospective power analysis was undertaken based on Cohen (1992) methodology recommending a sample size of 64 participants per group (for a between-subjects t-test, two-tailed) in order to attain a medium effect size $(d=0.5)$, with a recommended power level of 0.8 , and alpha of 0.05 . A medium effect size represents an effect likely to be visible to the observer (Cohen, 1992), and was accepted in the current study in the absence of available data to indicate the expected effect sizes of between-group differences on the relevant variables under consideration. 


\section{3 - RESULTS}

As indicated in Chapter 2 (Section 2.1), the study utilised a mixed qualitative (focus groups) and quantitative (survey) methodological approach in order both to explore the subject area in more detail and address specific hypotheses. Data for each part of the study are presented below, together with the rationale for chosen methods of analysis.

\subsection{ANALYSIS OF QUALITATIVE DATA}

Focus group data were analysed using framework analysis (Ritchie \& Spencer, 1994), a qualitative method originally developed for applied policy research but gaining popularity in health-related research (Lacey \& Luff, 2001). Framework analysis shares many of the common features of content or 'thematic' analysis, and provides a systematic and visible outline of stages of the analytic process. A primarily inductive approach in which categories and themes are developed directly from the data, this form of analysis also allows for inclusion of $a$ priori as well as emergent concepts in coding. The process involves five key stages (Table 4), which can either be undertaken in a linear fashion ${ }^{9}$ following data collection, or when data collection and analysis are undertaken concurrently.

\footnotetext{
${ }^{9}$ In this regard, Framework Analysis differs from a classical Grounded Theory approach in which a key feature is the identification of emergent themes through the simultaneous collection and analysis of data using the constant comparative method.
} 


\section{Table 4: Qualitative Analysis Outline}

\section{Key Stages of Framework Analysis:}

- Familiarisation: whole or partial transcription and reading of the data.

- Identifying a thematic framework: development of an initial coding framework both from a priori issues and from emerging issues identified during the familiaristation stage. The thematic framework should be developed and refined during subsequent stages of analysis.

- Indexing/Coding: the process of applying the thematic framework to the data, using numerical or textual codes to identify specific pieces of data which correspond to differing themes.

- Charting: creating charts of the data using headings from the thematic framework.

- Mapping and Interpretation: the search for patterns, associations, concepts, or explanations in the data, aided by visual schematics.

(Source: Lacey \& Luff, 2001, p.9)

Using Framework Analysis, focus group discussions were fully transcribed and identifying information removed. Transcripts were then read and re-read and an initial coding framework developed. The provisional thematic framework was further refined through an iterative process of indexing and charting of data, with continual reference to the original transcripts. Transcripts were indexed using numerical codes (relating to the thematic framework) to identify specific pieces of data (i.e. words or phrases) corresponding to different themes (Appendix 3.5). Where themes were identified which did not accord with the initial classification, new ones were developed. Thematic charts were created outlining major and constituent themes, and relationships within the data further explored by review of developing themes and direct quotations of relevance to each theme (Appendix 3.8). Data were continually re-assessed and re-coded in this manner until no new themes could be identified. Although group transcripts were analysed separately, results were subsequently integrated to facilitate an overall comparison (Powell \& Single, 
1996). Data were managed using NVIVO (Version 7; QSR International). A reflexive approach was adopted throughout, acknowledging the centrality of the researcher in the research process, with attempts made to differentiate participant description from researcher interpretation (Willig, 2001). Issues of quality control in relation to analysis of qualitative data are provided in Appendix 3.9.

\subsubsection{Identified Themes}

Themes emerging from the analysis of data from non-clinical participants were grouped into the following four clusters:

- Primary associations (attitudes to mental illness)

- Possible contributory factors impacting on attitudes

- Outcomes (i.e. health-behaviours and coping strategies)

- Broader implications for future service development

Once clusters of themes had been produced for the first group narrative, the coding process was then repeated for the clinical group discussion. During this process attempts were made to consider each group narrative afresh in the context of emerging themes, although the sequential nature of this stage of the analytic process invariably meant that the researcher was primed to certain aspects of the data (Smith et al., 1999). Table 5 outlines the final thematic framework used to code transcripts.

Eight superordinate themes emerged from the analysis of both transcripts. An overview of identified themes indicating areas of group convergence and divergence is presented in Appendix 3.10. Themes are described below and presented with selected quotations from coded transcripts (see Appendices 3.5a and 3.5b), with a more detailed discussion of results provided in Chapter 4. 


\section{Table 5: Thematic Framework - Attitudes to Mental IIIness}

\begin{tabular}{|c|c|}
\hline $\begin{array}{l}\quad \text { A PRIORI ISSUES } \\
\text { Meanings of Mental Illness } \\
\text { Attitudinal diversity } \\
\text { Experiences - direct and indirect } \\
\text { Reactions (e.g. fear; shame; } \\
\text { perceived dangerousness; } \\
\text { benevolence; self-sufficiency) } \\
\text { Health beliefs } \\
\text { Impact } \\
\text { Self-identity } \\
\text { Relationships } \\
\text { Participation/Activities } \\
\text { Engagement } \\
\text { Health and well-being } \\
\text { Health behaviours } \\
\text { Societal Perceptions } \\
\text { Positive and negative views (e.g. } \\
\text { sympathy; stigma) } \\
\text { Deficits in knowledge and } \\
\text { awareness } \\
\text { Prevalence of negative stereotypes } \\
\text { Mellowing of societal attitudes over } \\
\text { time } \\
\text { Ageing and Mental Illness } \\
\text { Intergenerational differences (in } \\
\text { perspectives) } \\
\text { Double stigmatisation } \\
\text { Discrimination } \\
\text { Understandability } \\
\text { Cohort beliefs } \\
\text { Self-stereotypes } \\
\text { Differing views of services } \\
\text { Treatment-Seeking/Coping } \\
\text { Sources of support } \\
\text { Willingness to seek help } \\
\text { Barriers to treatment (e.g. awareness } \\
\text { of services; provision; practical } \\
\text { barriers; ageism) } \\
\text { Expectations of treatment } \\
\text { Knowledge of treatments }\end{array}$ & 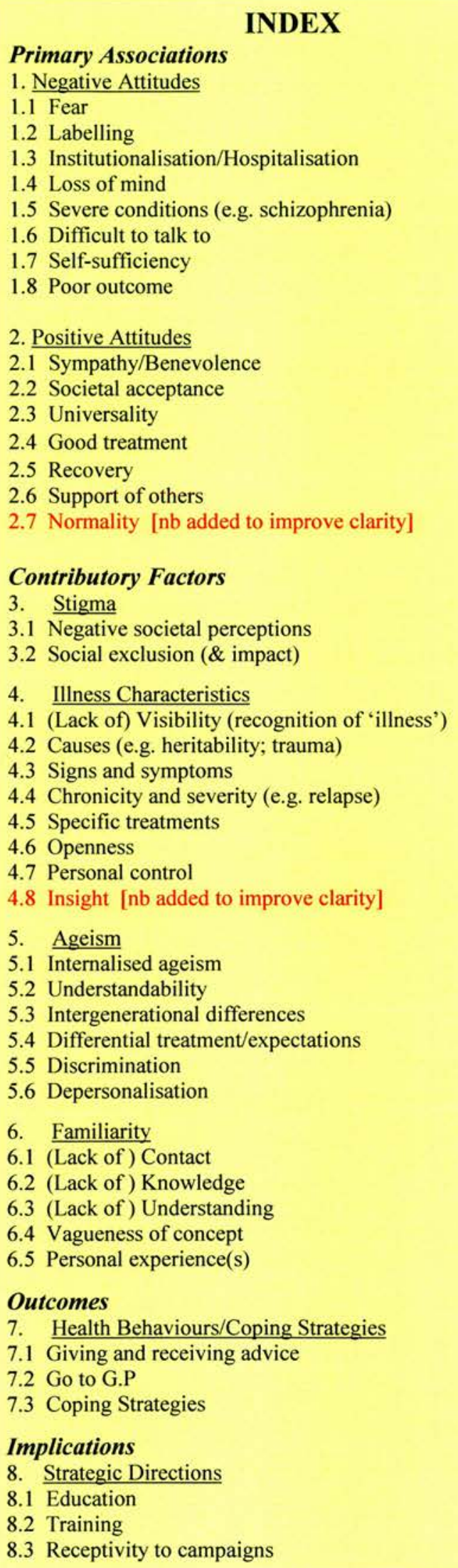 \\
\hline
\end{tabular}

\section{PRELIMINARY RESEARCH NOTES}

- 'Mental illness' difficult to define; 'illness' implies visibility and treatability.

- Equated with dementia; neurodegenerative conditions; 'loss of mind'.

- Internalised and explicit ageism; intergenerational differences in attitudes and reactions

- Indications of positive shift in attitudes towards societal acceptance. 


\subsubsection{Primary Associations}

\subsection{Negative Attitudes to Mental Illness}

In accordance with findings in the literature (e.g. Desforges et al., 1991), the concept of 'mental illness' generated strong negative reactions from participants, particularly those in the non-clinical group. Specifically, the term invoked fear related to a sense of individual vulnerability, and adverse outcome (e.g. potential institutionalisation). Links were also made with chronic and severe mental health conditions (e.g. such as schizophrenia), and particularly with loss of mental faculties and dementia:

'There is a certain fear you might lose some of your mental faculties and that bothers me.' (Non-clinical participant: p2 - lines 12-13).

' 'Just the fear it might happen to me.' (Non-clinical participant: p4 - line 11).

'I was terrified.' (Clinical participant: p6 - lines 15-16).

The social implications of labelling were highlighted, with some terms perceived as more socially acceptable than others:

'I think if they gave it another name..., people suffering with their nerves or suffering mental illness, I know which one I would rather have.'

(Non-clinical participant: p1 - lines 20-23).

A range of reactions to individuals with mental illness was noted, with participants indicating that they find it generally difficult to talk to individuals with mental health difficulties, and perceive mental health difficulties as being under individual control:

'And if you think of people having mental illness, I never know how to react with them.' (Non-clinical participant: p6; lines 27-29).

'I have to admit I used to feel like saying get yourself sorted out, you felt like giving her a smack across the backside.' (Non-clinical participant: p10 - lines 1-3). 
However, despite the prevalence of negative attitudes held by healthy participants, clinical participants tended not to express unfavourable views toward mental illness or individuals suffering from mental illness per se, tending instead to focus on poor expectations of recovery within the context of individual experiences of mental illness:

'in general understand there's a lot of people came here and actually got worse.

(Clinical participant: p2 - lines 13-15).

'Yeah, I'm not getting out of it.' (Clinical participant: p2 - line 26).

\subsection{Positive Attitudes to Mental Illness}

Negative attitudes were found to co-exist with positive attitudes towards mental illness in both groups. Participants expressed an array of positive sentiments including benevolence, a general awareness of increasing societal acceptance of mental illness, and increased social support (i.e. indicating a perception of positive attitudinal shift over time). Non-clinical participants acknowledged the universality of mental health problems and relative normality with which people with psychological difficulties are generally treated by others. Good quality (medical) treatment (regardless of age) and the possibility of recovery from mental illness were noted by participants from both groups:

When he was in ..., he was well looked after.' (Non-clinical participant: p14 -lines 27-28).

'I think people accept it more now, as an illness.' (Non-clinical participant: p6 - line 12).

'...eventually most of them do come round.' (Non-clinical participant; p15 - line 18). 
However positive attitudes were not impervious to the effects of ageist bias. Thus, non-clinical participants extended greater sympathy towards younger people with mental health problems than towards older people, suggesting the operation of internalised ageism and negative self-stereotypes:

'I would feel very sorry for the younger person, more sorry - I would be sorry for the older person too but I think, well they have got the rest of their life to go through.'

(Non-clinical participant: p9 - lines 11-13).

'Yes I think it is much sadder when you hear of someone in their 20s than somebody in their 80s.' (Non-clinical participant: p12 - lines 19-20).

\subsubsection{Contributory Factors}

\subsection{Stigma}

Non-clinical participants highlighted the prevalence of negative societal perceptions of mental illness and the continued stigma associated with psychiatric hospitals and treatment:

'I think the word 'mental' is a sort of stigma.' (Non-clinical participant: p1 - line 27).

'then when he went into [psychiatric hospital]... it was 'oh god he's in [...], fancy him being there etc. It was a down thing for him being there... people sort of said 'fancy him being in [...].' (Non-clinical participant: p4 - lines 31-34).

Clinical participants spoke of their own experiences and of the personal impact of labelling and social rejection. In particular, clinical participants noted that people often appeared not to know what to say or how to react around them, resulting in a variety of reactions ranging from verbal abuse to social exclusion: 
'are you happy to be talking to somebody, maybe just down in the bus stop and you just say "oh, I go to [psychiatric hospital] ... twice a week" and they say "oh, you're a nutter" ....'

(Clinical participant: p2 - lines 4-6).

'they started letting me get a weekend out, but naebody wanted me.'

(Clinical participant: p3 - lines 14-15).

These findings suggest that stigmatising attitudes continue to exist despite some indications of a positive societal shift toward greater acceptance (as indicated in Section 3.1.1.1.2).

\subsection{Illness Characteristics}

Attitudes appeared to reflect broader beliefs and perceptions about (the characteristics of) mental illness. For example, a recurrent theme in both focus group discussions concerned the ill-defined nature of 'mental illness', since 'illness' is most commonly equated with visible and treatable disease. The apparent lack of visible signs or symptoms of conditions such as depression, for example, was put forward by some participants as one possible reason for poor societal awareness, decreased tolerance and understanding of mental health difficulties (see Section 3.1.1.2.4). In apparent contrast to physical illness, mental illness was also considered more difficult to detect oneself due to lack of insight:

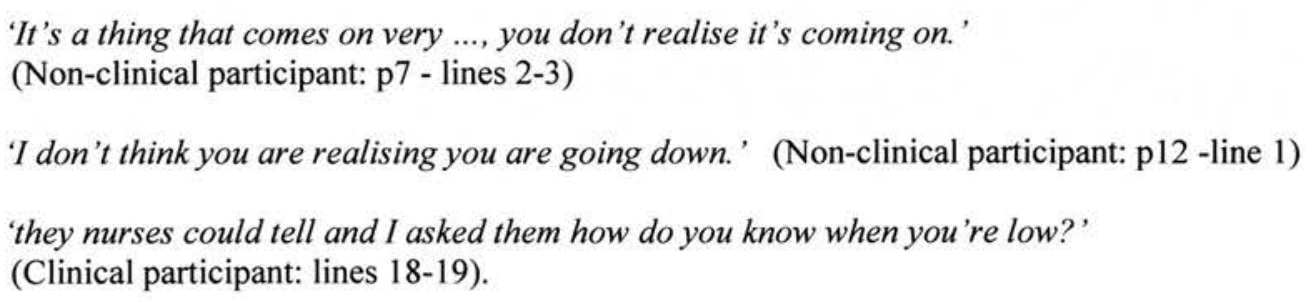


Attitudes also reflected individual beliefs regarding the perceived cause, course and controllability of illness with potential implications in terms of help-seeking behaviour, treatment responsivity, and outcome. Thus, participants from both groups expressed views that mental illness is largely hereditary, chronic and severe, and tends to be treated radically (e.g. by electroconvulsive therapy).

Non-clinical participants also expressed the idea that mental health problems are under personal control, perhaps reflecting broader cohort beliefs, cognitive dissonance (i.e. if an individual were to believe that mental illness is not controllable then it may enhance a sense of personal vulnerability, hence causing discomfort and leading to dissonance), and/or a general lack of direct personal experience of mental illness in this group:

'my attitude at that point until I got to know her better was, oh come on ....'

(Non-clinical participant: p10 - lines 23-24).

'well I used to think that come on you can stop.' (Non-clinical participant: p10 - line 26).

\subsubsection{3 $\underline{\text { Ageism }}$}

Implicit and explicit ageist attitudes were apparent throughout the non-clinical group narrative, although were not apparent in the clinical group discussion. Thus, in the non-clinical group narrative, depression was perceived as a normal part of growing older or 'the usual thing', and accepted as such by society. In terms of encounters with services, participants expected health professionals to underestimate their coping abilities, and to treat them in a patronising manner. One participant described a sense of depersonalisation, and of having her identity defined by her age: 
'it's quite sad really, because they are putting you into this category, you are not a person any more, you are just old ....' (Non-clinical participant: p7 - lines 22-23).

Intergenerational differences were also apparent in that younger adults were perceived to act differently towards older people with mental health problems:

'people's attitude to old people, younger people's attitude to old people is sometimes a bit awkward.' (Non-clinical participant: p7 - line 9-10).

\subsection{Familiarity}

Participants from both groups highlighted a general lack of societal (and personal) awareness and understanding of mental illness which they perceived as related to lack of individual experience and service contact. Participants reiterated the view that mental illness is difficult to define, often without apparent cause and with an illdefined prognosis:

'Some people have no contact with anybody like that so they don't know what it is really.'

(Non-clinical participant: pl - lines 13-14).

'a lot of people don't know what mental illness is, or understand it.'

(Non-clinical participant: p1 - lines 14-15).

'it's an illness just like any other illness... a lot of folk don't really understand that.'

(Non-clinical participant: pl - lines 16-17).

This points to a negative circular effect in which lack of personal experience of mental illness (either direct or indirect), deficits in public knowledge, lack of awareness, and lack of societal understanding contribute to and perpetuate negative stereotypes and discriminating attitudes. 


\subsubsection{Outcomes}

Participants varied in their (preferred or actual) choice of health behaviours and/or coping strategies. Non-clinical participants were most likely to give or seek advice, visit their general practitioner, or engage in a variety of coping strategies such as 'denial' or 'acceptance'. Clinical participants were much less vociferous about their own preferred strategies, tending instead to mention specific treatments such as taking of medications, although one participant did note the importance of remaining active throughout recovery:

'[being active]...that's the main thing, if you lie about in a hospital for 2-3 weeks you're not long in getting weak you know.' (Clinical participant: p8 - lines 4-5).

Engagement in proactive health behaviours such as help-seeking were viewed as necessarily dependent upon awareness of illness symptoms and attribution of symptoms to mental health problems.

\subsubsection{Implications: Possible Strategic Directions}

A number of suggestions for future strategic developments were made in relation to public education and staff training. For example, participants were explicit in their recognition of a societal need for education about mental illness, in order to enhance understanding and raise awareness:

'I think more education of the subject generally for us, for society, would help, would give a better understanding, would give us more insight.' (Non-clinical participant: p13 - lines 1-4). 
However, at the same time, several participants indicated that they were not aware of current high profile educational campaigns designed to combat stigma (e.g. 'See $\mathrm{Me}$ ' campaign; Scottish Executive 2003-2006), and were dubious about the capacity of such approaches to change attitudes. This suggests a general lack of awareness of public campaigns in some older people, perhaps reflecting the generic (i.e. nontargeted) focus of national public health approaches.

\subsubsection{SUMMARY AND MAPPING OF QUALITATIVE DATA}

Results from focus group analyses indicate that participants endorsed a broad range of attitudes to mental illness. Attitudes were more explicitly negative in those with no prior experience of mental health difficulties (i.e. non-clinical participants), and were related to individual fears of becoming mentally ill, of being institutionalised, or of loss of mental faculties, resulting in communicative barriers between people with and without mental health problems. Fears of mental illness appeared to be underpinned by perceived stigma, social rejection (endorsed by clinical participants), and a general lack of public awareness and understanding related to the 'invisibility' of conditions such as depression. In addition, age-related differences were perceived in terms of attributions of mental illness, such that mental illness in later life was perceived as 'understandable' by society, and particularly by health professionals, who were perceived to hold lower expectations of older peoples' coping resources and potential treatment responsivity. 
Interestingly, positive attitudes were found to co-exist with negative attitudes in participants from both groups. However, further discussion revealed that the positive attitudes of non-clinical participants were permeated by internalised ageism such that the focus of participants' benevolence was directed largely towards younger people with mental health problems, who were deemed more deserving of good mental health. Discussions of ageism were not apparent in the service users' narrative in which the focus was largely directed towards personal experiences of mental illness and expectations of recovery.

Figure 4 presents a schematic outline of identified themes, and the proposed associations between attitudes and other factors. As indicated above, attitudes are considered both to contribute to and be influenced by stigma, ageism (both external and internalised), lack of familiarity/knowledge of mental illness, and individual health beliefs (such as perceived illness cause and controllability). It is possible to hypothesise that the ways in which individuals encounter and make sense of these factors in light of personal history and experience may in turn impact upon decisions to engage in health-related behaviours and/or specific coping strategies such as denial or acceptance. The proposed links between attitudes and other factors brought to light by focus group discussions provide a basis for further exploration of the subject area through quantitative analysis. 


\section{Attitudes to Mental Illness}

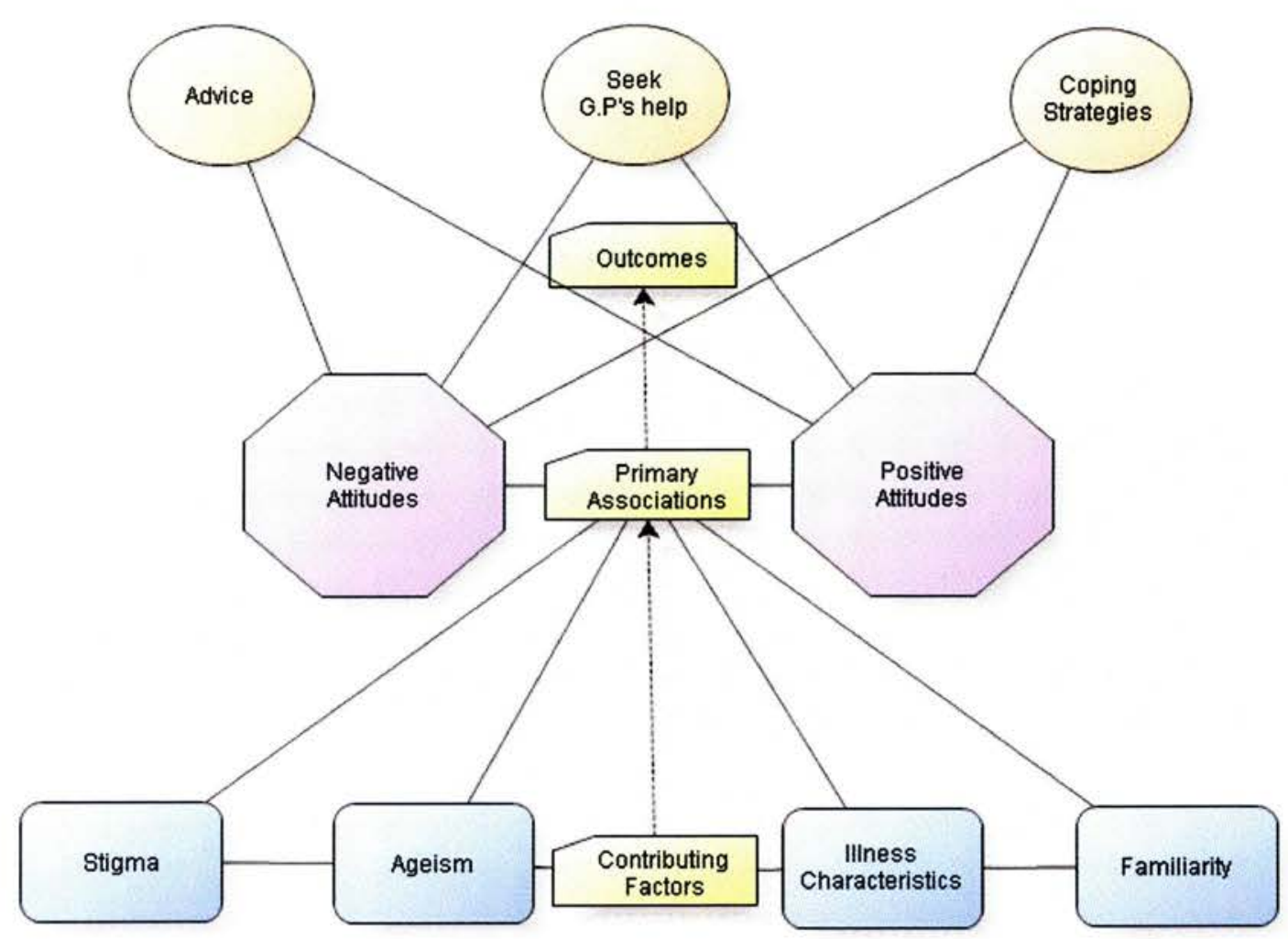

Figure 4: Mapping of Themes from Qualitative Analysis 


\subsection{ANALYSIS OF QUANTITATIVE DATA}

Data were analysed using the Statistical Package for the Social Sciences (SPSS; Version 14). An examination of minimum and maximum values, means and standard deviations was conducted to determine accuracy of data entry. Missing values were assigned a numerical value that identified these data within the data-set. Testing of the respective hypotheses was undertaken using parametric tests given that assumptions of variable measurement, normality of distribution and homogeneity of variance were met.

With respect to variable measurement, it is conventional in psychological research to treat ordinal data as interval if there is a sufficiently large range of possible scores for the dimensions to be analysed (Clark-Carter, 2004). The normality of item and scale distributions was examined with the aid of histograms and conversion of indexes of skewness and kurtosis to $z$ scores using the formulae in Field (2005). Tabachnik and Fidell (2001) recommend conventional but conservative alpha levels when evaluating the significance of skewness and kurtosis with small to moderate samples, thus $z$ scores with a recommended critical value of $+/-3.29(\alpha=0.001)$ were considered to indicate significant deviation from normality. Variables exhibiting significant skewness and/or kurtosis were considered for transformation, where appropriate. Homeogeneity of variance was assessed using Levene's test.

Where assumptions were met for parametric statistics, inter-group differences were assessed using independent $t$-tests, analysis of variance (i.e. ANOVA; MANOVA), or analysis of covariance (i.e. ANCOVA; MANCOVA) when controlling for 
possible confounding variables. Otherwise, non-parametric statistics were considered, providing assumptions for use of these tests were also met (Clark-Carter, 2004). Effect size and retrospective power calculations were undertaken for key comparisons.

The relationship between variables was examined using Chi-Square $\left(\chi^{2}\right)$ or Pearson's product-moment correlations (or the non-parametric Spearman's rank correlation coefficient). Further associations between independent and dependent variables were assessed using multiple regression analyses. Sample size for regression was calculated by the equation: $50+8 k$, where $k$ is the number of predictors (Green, 1991). For testing of individual predictors within the model, Green further recommends a sample size of $104+k$.

Alpha was set using the conventional criterion of $\mathrm{p} \leq 0.05$ for hypothesis-related comparisons, and adjusted to 0.001 for non-predicted tests to guard against the possibility of Type 1 error when undertaking multiple comparisons (Clark-Carter, 2004).

\subsubsection{DEMOGRAPHIC DATA}

Table 6 provides demographic data for the study sample. In total, 74 older people participated in the study, 50 in the non-clinical group (Group 1: 15 men, 35 women), and 24 in the clinical group (Group 2: 9 men, 15 women). Pearson's Chi-square analysis indicated that groups did not differ according to sex, with both samples predominantly female $\left(\chi_{(1)}^{2}=0.42, p=0.52\right)$. In addition, no inter-group differences were found with respect to marital status $\left(\chi_{(2)}^{2}=3.31, p=0.19\right)$, level of education 
$\left(\chi_{(2)}^{2}=0.69, \mathrm{p}=0.71\right)$ or living arrangements $\left(\chi_{(1)}^{2}=1.76, \mathrm{p}=0.19\right)$. However, significant differences were found between the groups in relation to subjective health $\left(\chi_{(2)}^{2}=7.28, \mathrm{p}=0.03\right)$, and past experience of mental illness $\left(\chi_{(2)}^{2}=43.97, \mathrm{p}<0.001\right)$. As indicated in Table 6, a larger proportion of participants in the non-clinical sample reported good health compared to the clinical sample, while a larger proportion of the clinical sample had prior direct and personal experience of mental illness than the non-clinical sample. Groups also differed in terms of age category $\left(\chi_{(1)}^{2}=6.43 ; \mathrm{p}=\right.$ 0.01 ), with 50 per cent of non-clinical participants over the age of 80 (oldest old) compared to only 18.2 per cent of participants in the clinical group.

Table 6: Demographic Data for Non-clinical and Clinical Samples

\begin{tabular}{|c|c|c|c|c|c|c|}
\hline \multirow[b]{2}{*}{ Demographic } & \multicolumn{2}{|c|}{$\begin{array}{c}\text { Non-clinical } \\
(\mathrm{N}=\mathbf{5 0})\end{array}$} & \multicolumn{2}{|c|}{$\begin{array}{l}\text { Clinical } \\
(\mathrm{N}=24)\end{array}$} & \multirow[t]{2}{*}{$\begin{array}{l}\text { Statistic } \\
\text { (df) }\end{array}$} & \multirow[t]{2}{*}{$\begin{array}{c}\text { Sig. } \\
\text { (2-tailed) }\end{array}$} \\
\hline & $N$ & $\%$ & $N$ & $\%$ & & \\
\hline $\begin{array}{l}\text { Gender } \\
\text { Male } \\
\text { Female }\end{array}$ & $\begin{array}{l}15 \\
35\end{array}$ & $\begin{array}{l}30.0 \\
70.0\end{array}$ & $\begin{array}{c}9 \\
15\end{array}$ & $\begin{array}{l}37.5 \\
62.5\end{array}$ & $\chi_{(1)}^{2}=0.42$ & $\mathrm{p}=0.52$ \\
\hline $\begin{array}{l}\text { Marital Status } \\
\text { Single/divorced } \\
\text { Married/Partnered } \\
\text { Widowed }\end{array}$ & $\begin{array}{c}6 \\
19 \\
25\end{array}$ & $\begin{array}{l}12.0 \\
38.0 \\
50.0\end{array}$ & $\begin{array}{l}6 \\
9 \\
7\end{array}$ & $\begin{array}{l}27.3 \\
40.9 \\
31.8\end{array}$ & $\chi_{(2)}^{2}=3.31$ & $\mathrm{p}=0.19$ \\
\hline $\begin{array}{l}\text { Education } \\
\text { Secondary school } \\
\text { Trade certificate } \\
\text { College/University }\end{array}$ & $\begin{array}{c}31 \\
3 \\
11\end{array}$ & $\begin{array}{c}68.9 \\
6.7 \\
24.4\end{array}$ & $\begin{array}{c}15 \\
1 \\
3\end{array}$ & $\begin{array}{c}78.9 \\
5.3 \\
15.8\end{array}$ & $\chi_{(2)}^{2}=0.69$ & $\mathrm{p}=0.71$ \\
\hline $\begin{array}{l}\text { Living arrangements } \\
\text { Living at home/with family } \\
\text { Living in residential care }\end{array}$ & $\begin{array}{c}41 \\
7 \\
\end{array}$ & $\begin{array}{l}85.4 \\
14.6 \\
\end{array}$ & $\begin{array}{c}23 \\
1\end{array}$ & $\begin{array}{c}95.8 \\
4.2\end{array}$ & $\chi_{(1)}^{2}=1.76$ & $\mathrm{p}=0.19$ \\
\hline $\begin{array}{l}\text { Health } \\
\text { Good } \\
\text { Fairly good } \\
\text { Not good } \\
\end{array}$ & $\begin{array}{c}17 \\
24 \\
7\end{array}$ & $\begin{array}{l}35.4 \\
50.0 \\
14.6\end{array}$ & $\begin{array}{c}2 \\
13 \\
8\end{array}$ & $\begin{array}{c}8.7 \\
56.5 \\
34.8\end{array}$ & $\chi_{(2)}^{2}=7.28$ & $p=0.03$ \\
\hline $\begin{array}{l}\text { Past experience } \\
\text { Direct } \\
\text { Indirect } \\
\text { None } \\
\end{array}$ & $\begin{array}{c}2 \\
10 \\
33\end{array}$ & $\begin{array}{c}4.4 \\
22.2 \\
73.3\end{array}$ & $\begin{array}{l}18 \\
3 \\
1\end{array}$ & $\begin{array}{c}81.8 \\
13.6 \\
4.5\end{array}$ & $\chi_{(2)}^{2}=43.97$ & $\mathrm{p}<0.001$ \\
\hline $\begin{array}{l}\text { Age category } \\
\text { Young-old }(<80 \text { years) } \\
\text { Old-old }(\geq 80 \text { years) }\end{array}$ & $\begin{array}{l}25 \\
25\end{array}$ & $\begin{array}{l}50.0 \\
50.0\end{array}$ & $\begin{array}{c}18 \\
4\end{array}$ & $\begin{array}{l}81.8 \\
18.2\end{array}$ & $\chi_{(1)}^{2}=6.43$ & $p=0.01$ \\
\hline
\end{tabular}

*Some categories collapsed to increase expected cell count. Missing data account for some cells falling short of total group sample size. 
The overall mean age of participants in the study was 77.63 years (SD 8.04). There was an observed difference in the mean age of the two groups, with the non-clinical group being older on average than the clinical group (i.e. 79.84 years, SD 8.05, and 72.59, SD 5.42, respectively). The larger inter-quartile range of the non-clinical group also indicates greater within-group variation (Figure 5).

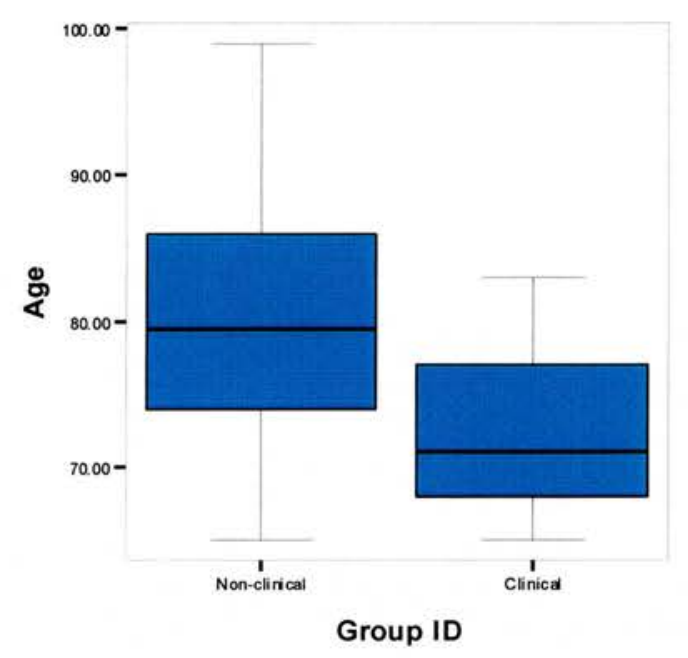

Figure 5: Comparison of Age in Non-clinical and Clinical Groups.

Although the distribution of age did not deviate significantly from normality in either group (see Appendices 4.1 and 4.2), unequal variances were found $\left(\mathrm{F}_{(1,70)}=4.90, p\right.$ $=0.03$ ). A Welch's $t$-test (where equal variances are not assumed) confirmed the mean age difference between the groups as significant $(t(70)=4.47, p<0.001)$ (Table 7). Bivariate correlations (Appendix 4.3) further indicated that age correlated significantly with at least two of the main study measures (BMHSS: Ageism; $r=.27$, $\mathrm{p}=0.02$, and AAQ: Physical; $r=.32, \mathrm{p}=0.007)$, therefore further inter-group analyses were undertaken with and without age as a covariate. 
Table 7: Mean Age of Non-clinical and Clinical Samples

\begin{tabular}{|c|c|c|c|c|c|c|c|c|}
\hline Variable & Group & $\mathbf{N}$ & Mean & SD & Median & $\begin{array}{c}\boldsymbol{t}^{*} \\
(\text { Sig. })\end{array}$ & $\begin{array}{c}\text { Mean diff } \\
(\mathbf{9 5} \% \mathbf{C I})\end{array}$ & $\mathbf{d f}$ \\
\hline \multirow{2}{*}{ Age } & $\begin{array}{c}\text { Non- } \\
\text { clinical }\end{array}$ & 50 & 79.84 & 8.05 & 79.50 & $\begin{array}{c}4.47 \\
(\boldsymbol{p}<\mathbf{0 . 0 0 1 )}\end{array}$ & $\begin{array}{c}7.25 \\
(4.00 \text { to 10.50) }\end{array}$ & 70 \\
\cline { 2 - 9 } & Clinical & $22^{+}$ & 72.59 & 5.42 & 71.00 & \multirow{2}{*}{. } \\
\hline
\end{tabular}

* Equal variances not assumed; Significance is two-tailed.

${ }^{+}$Two participants declined to give their age resulting in data for $n=22$.

\subsubsection{EXPLORATION OF DATA}

Summary descriptives of individual variables were reviewed. In general there were few missing values in the dataset. Almost all variables had missing values of less than 10 per cent, with the exception of Scottish National Survey items (where 'Don't know' responses were also assigned as missing values and account for inflated scores), several items on the BMHSS subscales (where missing values may reflect poor item construction and/or wording, such as the use of double-negatives), and Rame items relating to sexual activity (items 7 and 21), where missing values were higher than average ${ }^{10}$. Following guidelines set out by Winer (1971), missing values were therefore replaced, where appropriate, with the relevant mean variable scores on subsequent analyses. This procedure provides a conservative approach to missing values, and is recommended when the percentage of missing values is low (i.e. below 10 per cent).

A review of scale distributions indicated that, although a 'normal' (i.e. not significantly deviant) distribution was evident for several of the main study measures

\footnotetext{
${ }^{10}$ High missing values on sexual activity items is a common finding in the gerontological literature (e.g. Power et al., 2005), although may also reflect that a majority of the study sample was single/divorced or widowed.
} 
(i.e. BMHSS 'Stigma'; AAQ; Rame; RHBC), significant deviations from normality were observed for the BMHSS sub-scale 'Ageism', Understandability, and the GHQ12 (see Appendices 4.4; 4.5). A review of scale histograms of the latter three measures (Figure 6) indicates positively skewed and leptokurtic distributions (indicated by positive kurtosis $z$-scores) for BMHSS 'Ageism' and for Understandability, and a positively skewed and platykurtic distribution (indicated by negative kurtosis $z$-scores) for the GHQ-12. It is apparent for the 'Ageism' and Understandability scales that the skew indicates bunching of scores towards more positive attitudes towards mental illness in the overall sample. The positively skewed distribution of scores across the GHQ-12 is entirely consistent with findings in the literature (e.g. McLoughlin et al., 2005) for scoring of the GHQ-12 using the Likert method, although this method of scoring also reportedly leads to a less skewed distribution overall than the traditional GHQ (i.e. case ascertainment) method (Johnston et al., 1995).

The robustness of parametric tests even when some assumptions are violated has been noted by Clark-Carter (2004) who recommends use of non-parametric tests only if more than one assumption underlying a parametric test is violated. Accordingly, homogeneity of variance was also assessed for all study measures (Appendix 4.6). Only the Rame $\left(\mathrm{F}_{(1,72)}=7.90, \mathrm{p}=0.006\right)$ and the RHBC 'Cognitive Affective' subscale $\left(\mathrm{F}_{(1,72)}=4.23, \mathrm{p}=0.04\right)$ exhibited unequal variances. Thus, as none of the study measures exhibited both significant deviations from normality and lack of homogeneity of variance, transformations were not undertaken and at least one assumption necessary for parametric tests was considered to be met. 


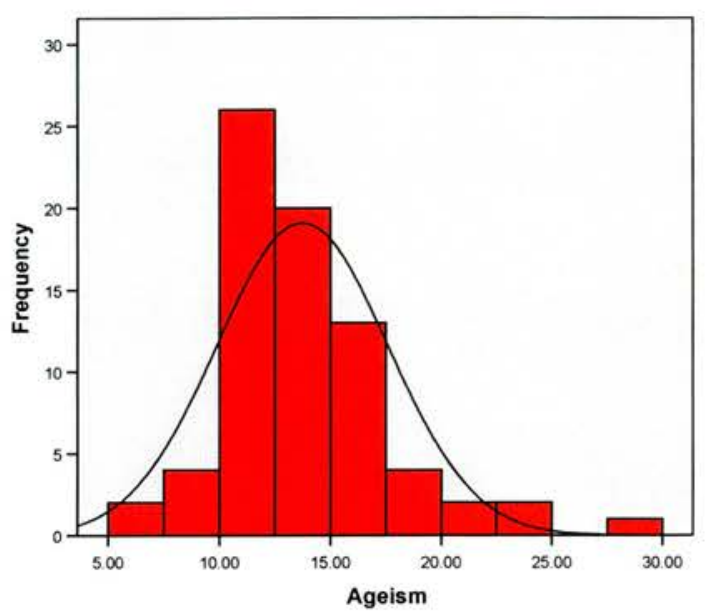

BMHSS: Ageism

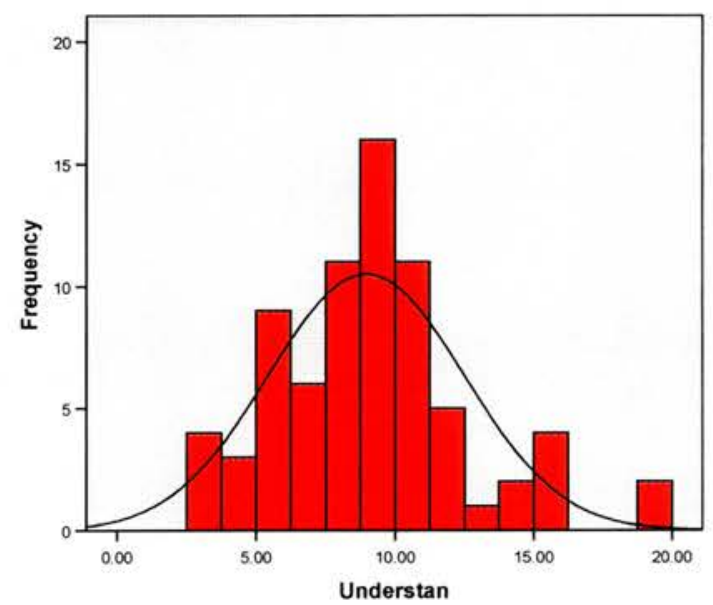

Understandability
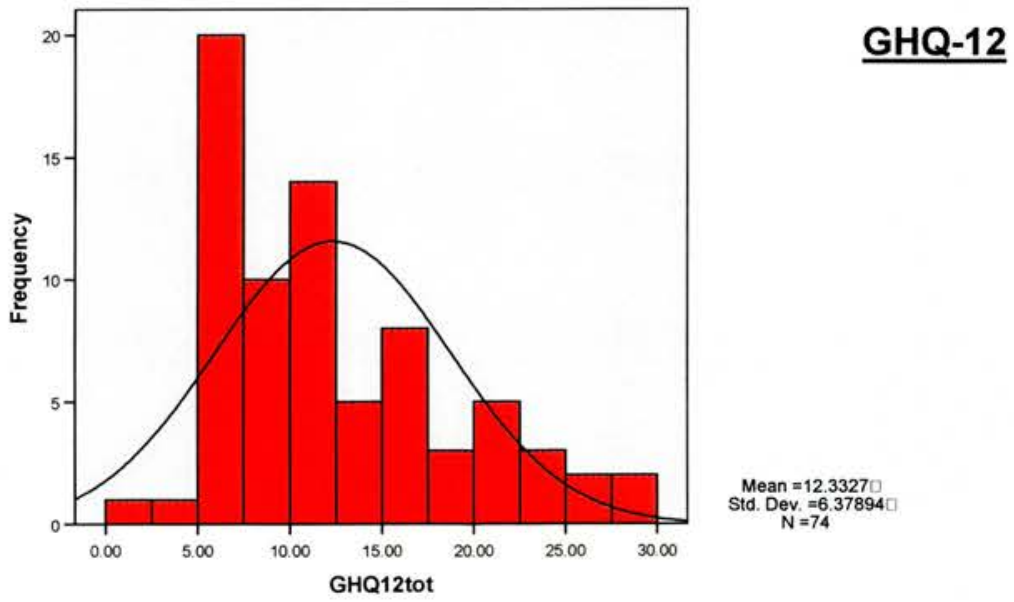

Figure 6: Histograms of Scale Distributions 
Finally, to assess internal reliability, Cronbach's alpha $(\alpha)$ co-efficients were computed for each of the main study scales. Although a value of Cronbach's alpha of 0.7 and above is generally considered acceptable within the empirical literature, Kline (1999) advocates that values below 0.7 can realistically be expected when dealing with psychological phenomena because of the diversity of the constructs being measured. Table 8 indicates Cronbach's alpha co-efficients were reasonably high (i.e. above 0.6) across most study measures, with the exception of the Understandability scale $(\alpha=0.39)$, and the 'Stigma' sub-scale of the BMHSS $(\alpha=$ 0.51). Field (2005) argues that in a reliable scale, all items should correlate above 0.3 with the total scale score. As indicated in Appendix 4.7, Items 1 and 3 of the Understandability measure demonstrated poor corrected item-total correlations, however, as the scale comprises only 3 items (i.e. the lowest acceptable minimum number of items to compute internal reliability), these results should be interpreted cautiously. Similarly, items 6 and 11 of the BMHSS 'Stigma' sub-scale demonstrated corrected item-total correlations below 0.3 , with a slightly improved alpha co-efficient evident on deletion of item 6 (i.e. 'I would see a psychologist because normal people go to psychotherapy'). Although the BMHSS and Understandability measures have been developed in older adult populations, neither measure has been comprehensively psychometrically tested, thus placing limitations on interpretation of subsequent analyses incorporating either measure. 
Table 8: Scale Reliability Co-efficients

\begin{tabular}{|l|c|c|c|}
\hline \multicolumn{1}{|c|}{ Scale } & N items & N cases * & Alpha co-efficient $^{+}$ \\
\hline BMHSS: & 6 & 74 & 0.74 \\
Ageism & 6 & 74 & 0.51 \\
Stigma & & & $0.39^{++}$ \\
\hline & 3 & 74 & 0.80 \\
Understandability & 24 & 74 & 0.71 \\
\hline AAQ: & 8 & 74 & 0.79 \\
Growth & 8 & 74 & 0.61 \\
Loss & 8 & 74 & 0.80 \\
Physical & 20 & 74 & 0.90 \\
\hline RAME & 12 & 74 & 0.70 \\
GHQ-12 & 21 & 74 & 0.63 \\
\hline RHBC: & 15 & 74 & 0.75 \\
Health-Action & 6 & 74 & \\
Cognitive-Affective & \multicolumn{2}{|l}{} \\
\hline
\end{tabular}

* Missing values replaced by series mean.

+ Values of 0.5 and above are considered acceptable in social science data (Kline, 1999).

++ Scale comprises only 3 items so alpha should be interpreted with caution.

\subsubsection{HYPOTHESIS-RELATED DATA}

Analyses are presented by hypothesis and separated into two main sections. First, data are presented for between-group analyses in relation to each of the main study measures being addressed. Second, specific hypothesis-related analyses are presented.

3.2.3.1 Hypothesis 1: Older people will endorse more negative attitudes to mental illness than younger people. Intra-cohort attitudes will differ by age and gender.

\subsection{Between-group Analyses: National Survey Items}

Analysis of Covariance (ANCOVA), with age as the covariate, was used to establish whether responses differed in terms of general attitudes to mental illness (as assessed 
by responses to the nine items from the National Survey; Scottish Executive, 2004) according to clinical status. Univariate analyses revealed that, after adjusting for the effect of age on outcome, there was no significant effect of clinical status on attitudes to mental illness on eight out of nine attitudinal items (Table 9). A significant intergroup difference was found for item 8 (i.e. 'People with mental health problems should have the same rights as anyone else'). An examination of mean scores and standard deviations for non-clinical and clinical samples on this variable indicated that the clinical group expressed more lenient views regarding the rights of individuals with mental health problems than participants in the non-clinical group $\left(F_{(1,69)}=4.81, p=0.03\right)$. The effect size of the between-group difference on item 8 was in the medium range $(d=-0.56)$, although observed power was substantially below that desired $(\mathrm{P}=0.58)$. Similarly, effect sizes for all remaining variables ranged from small (i.e. 0.2) to medium (i.e. 0.5), with retrospective power calculations indicating insufficient power to detect such effects. As this increases the possibility of a Type II error, results should be interpreted cautiously.

When the effect of age was considered in the analysis, a significant inter-group difference was found in relation to item 4 (i.e. 'I would find it hard to talk to someone with mental health problems'; $\left.\mathrm{F}_{(1,69)}=6.68, \mathrm{p}=0.01\right)$, indicating that clinical (i.e. younger) participants find it generally less difficult to talk to someone with mental health problems than non-clinical (i.e. older) participants (Appendix 4.8). However, as noted above, when the effect of age was controlled, no significant inter-group differences were observed on this variable. In addition, Analysis of Variance (ANOVA) revealed no inter-group differences on any of the National Survey attitudinal items when analyses were re-run without adjusting for age 
(Appendix 4.9). These results therefore indicate that clinical participants do not endorse more negative general attitudes to mental illness than non-clinical participants.

\section{Table 9: Between-group Comparison of Attitudes to National Survey Items (with age as covariate)}

\begin{tabular}{|c|c|c|c|c|c|c|c|}
\hline Survey Item & Group & $\begin{array}{c}\text { Mean } \\
(1-5)\end{array}$ & $\begin{array}{l}\text { Std } \\
\text { Dev }\end{array}$ & $95 \%$ CI & d.f. & $\begin{array}{c}\text { F } \\
\text { (Sig.) }\end{array}$ & $\begin{array}{c}\text { Effect } \\
\text { Size }^{+} \& \\
\text { Observed } \\
\text { Power }\end{array}$ \\
\hline \multirow{2}{*}{$\begin{array}{l}\text { 1. If I was suffering from } \\
\text { mental health problems I } \\
\text { wouldn't want people } \\
\text { knowing about it }\end{array}$} & $\begin{array}{l}\text { Non-clinical } \\
(\mathrm{N}=50)\end{array}$ & 2.77 & 1.15 & $2.42-3.11$ & \multirow[t]{2}{*}{1,69} & \multirow{2}{*}{$\begin{array}{c}1.33 \\
(p=0.30)\end{array}$} & \multirow{2}{*}{$\begin{array}{c}d=0.31 \\
\mathrm{P}=0.21\end{array}$} \\
\hline & $\begin{array}{l}\text { Clinical Group } \\
\left(\mathrm{N}=\mathbf{2 2}^{++}\right)\end{array}$ & 3.14 & 1.25 & $2.61-3.69$ & & & \\
\hline \multirow{2}{*}{$\begin{array}{l}\text { 2. The public should be } \\
\text { better protected from } \\
\text { people with mental health } \\
\text { problems }\end{array}$} & $\begin{array}{l}\text { Non-clinical } \\
(\mathrm{N}=50)\end{array}$ & 2.90 & 1.34 & $2.53-3.31$ & \multirow[t]{2}{*}{1,69} & \multirow{2}{*}{$\begin{array}{c}0.34 \\
(p=0.56)\end{array}$} & \multirow{2}{*}{$\begin{array}{c}d=0.21 \\
\mathrm{P}=0.09\end{array}$} \\
\hline & $\begin{array}{l}\text { Clinical Group } \\
(\mathrm{N}=\mathbf{2 2})\end{array}$ & 3.18 & 1.33 & $2.53-3.75$ & & & \\
\hline \multirow{2}{*}{$\begin{array}{l}\text { 3. Anyone can suffer } \\
\text { from mental health } \\
\text { problems }\end{array}$} & $\begin{array}{l}\text { Non-clinical } \\
(\mathrm{N}=50)\end{array}$ & 1.21 & 0.40 & $1.04-1.34$ & \multirow[t]{2}{*}{1,69} & \multirow{2}{*}{$\begin{array}{c}2.58 \\
(p=0.11)\end{array}$} & \multirow{2}{*}{$\begin{array}{c}d=0.29 \\
\mathrm{P}=0.35\end{array}$} \\
\hline & $\begin{array}{l}\text { Clinical Group } \\
(\mathbf{N}=\mathbf{2 2})\end{array}$ & 1.36 & 0.73 & $1.18-1.66$ & & & \\
\hline \multirow{2}{*}{$\begin{array}{l}\text { 4. I would find it hard to } \\
\text { talk to someone with } \\
\text { mental health problems }\end{array}$} & $\begin{array}{l}\text { Non-clinical } \\
(\mathrm{N}=50)\end{array}$ & 3.49 & 1.17 & $3.27-3.91$ & \multirow[t]{2}{*}{1,69} & \multirow{2}{*}{$\begin{array}{c}0.58 \\
(p=0.45)\end{array}$} & \multirow{2}{*}{$\begin{array}{r}d=0.49 \\
\mathrm{P}=0.12\end{array}$} \\
\hline & $\begin{array}{l}\text { Clinical Group } \\
(\mathrm{N}=\mathbf{2 2})\end{array}$ & 4.06 & 1.07 & $3.33-4.33$ & & & \\
\hline \multirow{2}{*}{$\begin{array}{l}\text { 5. People are generally } \\
\text { caring and sympathetic to } \\
\text { people with mental health } \\
\text { problems }\end{array}$} & $\begin{array}{l}\text { Non-clinical } \\
(\mathrm{N}=50)\end{array}$ & 2.84 & 1.18 & $2.50-3.23$ & \multirow[t]{2}{*}{1,69} & \multirow{2}{*}{$\begin{array}{c}0.34 \\
(p=0.56)\end{array}$} & \multirow{2}{*}{$\begin{array}{l}d=-0.09 \\
P=0.09\end{array}$} \\
\hline & $\begin{array}{l}\text { Clinical Group } \\
(\mathbf{N}=\mathbf{2 2})\end{array}$ & 2.73 & 1.35 & $2.10-3.23$ & & & \\
\hline \multirow{2}{*}{$\begin{array}{l}\text { 6. People with mental } \\
\text { health problems are often } \\
\text { dangerous }\end{array}$} & $\begin{array}{l}\text { Non-clinical } \\
(\mathrm{N}=50)\end{array}$ & 3.30 & 1.14 & $2.98-3.65$ & \multirow[t]{2}{*}{1,69} & \multirow{2}{*}{$\begin{array}{c}0.82 \\
(\mathrm{p}=0.37)\end{array}$} & \multirow{2}{*}{$\begin{array}{l}d=-0.30 \\
\mathrm{P}=0.15\end{array}$} \\
\hline & $\begin{array}{l}\text { Clinical Group } \\
(\mathbf{N}=\mathbf{2 2})\end{array}$ & 3.65 & 1.16 & $3.09-4.14$ & & & \\
\hline \multirow{2}{*}{$\begin{array}{l}\text { 7. The majority of people } \\
\text { with mental health } \\
\text { problems recover }\end{array}$} & $\begin{array}{l}\text { Non-clinical } \\
(\mathrm{N}=50)\end{array}$ & 2.81 & 0.90 & $2.49-3.07$ & \multirow[t]{2}{*}{1,69} & \multirow{2}{*}{$\begin{array}{c}0.21 \\
(p=0.65)\end{array}$} & \multirow{2}{*}{$\begin{array}{l}d=-0.23 \\
P=0.07\end{array}$} \\
\hline & $\begin{array}{l}\text { Clinical Group } \\
(\mathbf{N}=\mathbf{2 2})\end{array}$ & 2.58 & 1.16 & $2.20-3.10$ & & & \\
\hline \multirow{2}{*}{$\begin{array}{l}\text { 8. People with mental } \\
\text { health problems should } \\
\text { have the same rights as } \\
\text { anyone else }\end{array}$} & $\begin{array}{l}\text { Non-clinical } \\
(\mathrm{N}=\mathbf{5 0})\end{array}$ & 1.70 & 0.88 & $1.48-1.94$ & \multirow[t]{2}{*}{1,69} & \multirow{2}{*}{$\begin{array}{c}4.81 \\
(p=\mathbf{0 . 0 3})\end{array}$} & \multirow{2}{*}{$\begin{array}{l}d=-0.56 \\
\mathrm{P}=0.58\end{array}$} \\
\hline & $\begin{array}{l}\text { Clinical Group } \\
(\mathbf{N}=\mathbf{2 2})\end{array}$ & 1.26 & 0.43 & $0.87-1.58$ & & & \\
\hline \multirow{2}{*}{$\begin{array}{l}\text { 9. People with mental } \\
\text { health problems are } \\
\text { largely to blame for their } \\
\text { own condition }\end{array}$} & $\begin{array}{l}\text { Non-clinical } \\
(\mathrm{N}=\mathbf{5 0})\end{array}$ & 4.19 & 1.22 & $3.91-4.57$ & \multirow[t]{2}{*}{1,69} & \multirow{2}{*}{$\begin{array}{c}0.25 \\
(p=0.62)\end{array}$} & \multirow{2}{*}{$\begin{array}{c}d=0.28 \\
\mathrm{P}=0.08\end{array}$} \\
\hline & $\begin{array}{l}\text { Clinical Group } \\
(\mathrm{N}=22)\end{array}$ & 4.51 & 0.91 & $3.89-4.91$ & & & \\
\hline
\end{tabular}

* Mean score after missing values replaced.

+ Calculated according to Cohen's d formula (see Clark-Carter, 2004, p. 181). Partial eta-squared values are provided in Appendix 4.8 .

${ }^{++}$two participants declined to give their age resulting in data for $n=22$ for analysis of covariance (with age as covariate). 


\subsection{Comparison of Attitudes to Mental Illness in Younger and Older People}

To compare the views of older and younger people to mental illness, the proportion of participants aged $45-54^{11}$ from the National Survey study (Scottish Executive, 2004) agreeing with each of the nine general attitudinal statements was compared to the proportion of participants in the current study aged 65 and over also expressing agreement (Table 10). Results indicate that, for the most part, older and younger participants held broadly similar attitudes in relation to mental illness and endorsed a range of positive and negative views. For example, over 95 per cent of both samples agreed with the statement that 'Anyone can suffer from mental health problems' (Item 3), and more than one-third from each group also reported expectations of recovery from mental illness (Item 7). Over 40 per cent of each sample indicated that they would not want other people to know if they were suffering from mental health difficulties. However, less than one-quarter of the older adult sample, and less than one-sixth of the younger adult sample, agreed that 'People with mental health problems are often dangerous' (Item 6). In terms of inter-group differences, while a larger proportion of older than younger participants agreed with the statement in Item 2 proposing better protection for the public from people with mental health problems (i.e. endorsed a negative attitude; $\chi 2_{(1)}=4.50, p=0.03 ; \mathrm{phi}=-0.12, \mathrm{p}=0.03$ ), more older people also agreed with the statement in Item 5 that people are generally sympathetic and caring towards those who are mentally ill (i.e. endorsed a positive attitude; $\left.\chi_{(1)}^{2}=7.07, \mathrm{p}=0.008 ; \mathrm{phi}=-0.15, \mathrm{p}=0.008\right)$. A difference was also observed on Item 9 (i.e. 'People with mental health problems are largely to blame for their own condition'), with a slightly larger proportion of older people agreeing

\footnotetext{
${ }^{11}$ Requests for raw data for the 45-54 year sample, and for the 55-74 year age group, were declined by the Scottish Executive.
} 
with this statement. However, the effect size for all items was small, and the analysis underpowered.

\section{Table 10: Comparison of Younger and Older People's Attitudes to Mental Illness}

\begin{tabular}{|c|c|c|c|c|c|c|c|}
\hline \multirow[t]{2}{*}{$\begin{array}{l}\text { National } \\
\text { Survey } \\
\text { Item }\end{array}$} & \multicolumn{2}{|c|}{$\begin{array}{l}\text { Proportion of } \\
\text { 45-54 year } \\
\text { group } \\
\text { "agreeing"* } \\
(\mathrm{N}=237)\end{array}$} & \multicolumn{2}{|c|}{$\begin{array}{l}\text { Proportion of } \\
\text { current study } \\
\text { sample } \\
\text { (i.e. } 65 \text { plus) } \\
\text { "agreeing"* } \\
(\mathrm{N}=74)\end{array}$} & \multirow[t]{2}{*}{$\begin{array}{l}\text { Statistic } \\
\text { (df) }\end{array}$} & \multirow[t]{2}{*}{$\begin{array}{c}\text { Sig. } \\
\text { (2-tailed) }\end{array}$} & \multirow[t]{2}{*}{$\begin{array}{l}\text { Effect Size }(\omega) \\
\sqrt{\frac{\chi^{2}}{N}}\end{array}$} \\
\hline & $\mathbf{N}$ & $\%$ & $\mathbf{N}$ & $\%$ & & & \\
\hline 1 & 111 & 47 & 30 & 41 & $\chi_{(1)}^{2}=0.56$ & $p=0.46$ & 0.04 \\
\hline 2 & 54 & 23 & 26 & 35 & $\chi_{(1)}^{2}=4.50$ & $p=0.03$ & 0.12 \\
\hline 3 & 232 & 98 & 71 & 96 & $\chi_{(1)}^{2}=0.85$ & $\mathrm{p}=0.36^{+}$ & 0.05 \\
\hline 4 & 33 & 14 & 14 & 19 & $\chi_{(1)}^{2}=1.10$ & $p=0.30$ & 0.06 \\
\hline 5 & 78 & 33 & 37 & 50 & $\chi_{(1)}^{2}=7.07$ & $p=0.008$ & 0.15 \\
\hline 6 & 33 & 14 & 17 & 23 & $\chi_{(1)}^{2}=3.42$ & $p=0.06$ & 0.10 \\
\hline 7 & 113 & 48 & 30 & 41 & $\chi_{(1)}^{2}=1.16$ & $p=0.28$ & 0.06 \\
\hline 8 & 208 & 88 & 64 & 87 & $\chi_{(1)}^{2}=0.08$ & $p=0.77$ & 0.02 \\
\hline 9 & 9 & 4 & 7 & 10 & $\chi_{(1)}^{2}=3.71$ & $p=0.05^{++}$ & 0.11 \\
\hline
\end{tabular}

* Calculated by collapsing response categories 'Strongly agree' and 'Tend to agree'.

+ Fisher's Exact Test also calculated as $2 \times 2$ and low expected frequencies in $50 \%$ of cells. Fisher's Exact Test (Item 3: $d f=1, p=0.40$ ).

${ }^{++}$Item 9 reached borderline significance, however Fisher's Exact Test was calculated due to low expected frequencies in 25\% of cells and was not significant (Item 9: $d f=1, p=0.06$ (one-tailed).

Overall, these results do not support Hypothesis 1 that older people are inherently more negative in their attitudes towards mental illness than younger people (i.e. it is not possible to reject the null hypothesis of no significant difference between the age groups in terms of attitudes), although findings should be interpreted within the context of statistical limitations (e.g. small sample size etc.). However, in the National Survey (Scottish Executive, 2004) comparisons were made between participants aged 45-54 and those aged 75 and over, and it is possible, as has 
previously been suggested, that the oldest old endorse more negative views than the young old. To test this prediction, intra-cohort analyses were undertaken to compare the views of young-old (i.e. age 65-79) and old-old participants (i.e. aged 80 plus) across the entire current study sample. Results are shown in Table 11.

\section{Table 11: Comparison of Young-old and Old-old Attitudes to Mental IIIness}

\begin{tabular}{|c|c|c|c|c|c|c|c|}
\hline \multirow[b]{2}{*}{$\begin{array}{l}\text { National Survey } \\
\text { Item }{ }^{*}\end{array}$} & \multicolumn{2}{|c|}{$\begin{array}{l}\text { Young-Old } \\
(\mathrm{N}=43)\end{array}$} & \multicolumn{2}{|c|}{$\begin{array}{c}\text { Old-Old } \\
(\mathrm{N}=29)\end{array}$} & \multirow[t]{2}{*}{$\begin{array}{l}\text { Statistic } \\
\text { (df) }\end{array}$} & \multirow[t]{2}{*}{$\begin{array}{c}\text { Sig. } \\
\text { (2-tailed) }\end{array}$} & \multirow{2}{*}{$\begin{array}{c}\text { Effect } \\
\text { Size }(\omega) \\
\sqrt{\frac{\chi^{2}}{\mathrm{~N}}}\end{array}$} \\
\hline & $\begin{array}{c}\text { Agree } \\
N \\
(\%)\end{array}$ & $\begin{array}{c}\text { Disagree } \\
N \\
(\%)\end{array}$ & $\begin{array}{c}\text { Agree } \\
N \\
(\%)\end{array}$ & $\begin{array}{c}\text { Disagree } \\
N \\
(\%)\end{array}$ & & & \\
\hline 1 & $\begin{array}{c}19 \\
(59.4)\end{array}$ & $\begin{array}{c}13 \\
(40.6)\end{array}$ & $\begin{array}{c}10 \\
(58.8)\end{array}$ & $\begin{array}{c}7 \\
(41.2)\end{array}$ & $\mathrm{X}_{(1)}^{2}=0.01$ & $\mathrm{p}=0.97$ & 0.005 \\
\hline 2 & $\begin{array}{c}15 \\
(48.4)\end{array}$ & $\begin{array}{c}16 \\
(51.6)\end{array}$ & $\begin{array}{c}11 \\
(52.4)\end{array}$ & $\begin{array}{c}10 \\
(47.6)\end{array}$ & $\mathrm{X}_{(1)}^{2}=0.08$ & $\mathrm{p}=0.78$ & 0.04 \\
\hline 3 & $\begin{array}{c}42 \\
(97.7)\end{array}$ & $\begin{array}{c}1 \\
(2.3)\end{array}$ & $\begin{array}{c}27 \\
(39.1)\end{array}$ & $\begin{array}{c}0 \\
(0)\end{array}$ & $X_{(1)}^{2}=0.64$ & $\mathrm{p}=0.43^{+}$ & 0.10 \\
\hline 4 & $\begin{array}{c}3 \\
(9.4)\end{array}$ & $\begin{array}{c}29 \\
(90.6)\end{array}$ & $\begin{array}{c}10 \\
(43.5)\end{array}$ & $\begin{array}{c}13 \\
(56.5)\end{array}$ & $\mathrm{X}_{(1)}^{2}=8.62$ & $\mathrm{p}=0.003^{+}$ & 0.40 \\
\hline 5 & $\begin{array}{c}23 \\
(63.9)\end{array}$ & $\begin{array}{c}13 \\
(36.1)\end{array}$ & $\begin{array}{c}14 \\
(53.8)\end{array}$ & $\begin{array}{c}12 \\
(46.2)\end{array}$ & $\mathrm{X}_{(1)}^{2}=0.63$ & $p=0.43$ & 0.10 \\
\hline 6 & $\begin{array}{c}8 \\
(27.6)\end{array}$ & $\begin{array}{c}21 \\
(72.4)\end{array}$ & $\begin{array}{c}9 \\
(42.9)\end{array}$ & $\begin{array}{c}12 \\
(57.1)\end{array}$ & $\mathrm{X}_{(1)}^{2}=1.27$ & $p=0.26$ & 0.16 \\
\hline 7 & $\begin{array}{c}17 \\
(73.9)\end{array}$ & $\begin{array}{c}6 \\
(26.1)\end{array}$ & $\begin{array}{c}12 \\
(52.2)\end{array}$ & $\begin{array}{c}11 \\
(47.8)\end{array}$ & $\mathrm{X}_{(1)}^{2}=2.33$ & $\mathrm{p}=0.13$ & 0.23 \\
\hline 8 & $\begin{array}{c}38 \\
(95.0)\end{array}$ & $\begin{array}{c}2 \\
5.0) \\
\end{array}$ & $\begin{array}{c}25 \\
(96.2)\end{array}$ & $\begin{array}{c}1 \\
(3.8)\end{array}$ & $\mathrm{X}_{(1)}^{2}=0.05$ & $\mathrm{p}=0.83^{+}$ & 0.03 \\
\hline 9 & $\begin{array}{c}4 \\
(10.8)\end{array}$ & $\begin{array}{c}33 \\
(89.2)\end{array}$ & $\begin{array}{c}3 \\
(13.0) \\
\end{array}$ & $\begin{array}{c}20 \\
(87.0)\end{array}$ & $\mathrm{X}_{(1)}^{2}=0.07$ & $\mathrm{p}=0.79$ & 0.03 \\
\hline
\end{tabular}

* Response categories collapsed to agree /disagree to increase expected cell count.

+ Fisher's Exact Test also calculated as $2 \times 2$ and low expected frequencies in $50 \%$ of cells. Fisher's Exact Test (Item 3: $d f=1, p=0.61$ ); Fisher's Exact Test (Item 4: $d f=1, p=0.004$ ); Fisher's Exact Test (Item 8: $d f=1, p=0.66$ )

Overall, the hypothesis that the oldest-old participants endorse more negative attitudes than younger-old participants was not supported. Results indicate only one attitudinal item (Item 4$)$ significantly differentiated the age groups $\left(\chi_{(1)}^{2}=8.62, p=\right.$ 0.003; phi $=-0.40, \mathrm{p}=0.003$ ). A review of frequencies for Item 4 (i.e. 'I would find it hard to talk to someone with mental health problems') indicates that a larger 
proportion of participants in the oldest-old age group than in the younger-old group agreed with this statement. A medium effect size was found, and power to detect this effect was high $(\mathrm{P}=0.84)$.

Finally, to assess the impact of gender on attitudes to mental illness, a series of Pearson's Chi-Square analyses were undertaken across the whole sample. Only Item 1 ('If I was suffering from mental health problems, I wouldn't want people knowing about it' $)$ approached borderline significance $\left(\chi_{(1)}^{2}=3.96, p=0.05 ;\right.$ phi $=0.28, p=$ 0.05), with a larger proportion of females than males disagreeing with this statement (51.7\% and $23.8 \%$ respectively; Appendix 4.10$)$. No other significant differences in attitudes in relation to gender were found. Thus, in contrast to findings by the National Survey (Scottish Executive, 2004), it appears that men in this study sample did not hold more negative attitudes towards mental illness than women.

3.2.3.2 Hypothesis 2: Participants currently in receipt of mental health services will endorse more positive attitudes to mental illness than those not in receipt of services. Those with prior experience of mental illness/mental health services will endorse more positive attitudes than those without such experience.

\subsection{Between-group Analyses: Attitudes to Mental Illness in Later Life}

A between-groups Multivariate Analysis of Covariance (MANCOVA), with age as the covariate, was conducted to establish whether scores on measures assessing attitudes to mental illness in later life (i.e. BMHSS 'Ageism', 'Stigma'; Understandability) significantly differed between non-clinical and clinical samples, with more negative attitudes proposed in the non-clinical group. Multivariate tests 
indicated that participant status (i.e. clinical or non-clinical) was not a significant factor in the model $\left(\mathrm{F}_{(3,65)}=0.70, \mathrm{p}=0.56\right)$. Sex was also entered as a betweenparticipants fixed factor and was not significant $\left(\mathrm{F}_{(3,65)}=1.25, \mathrm{p}=0.30\right)$. In addition, the interaction between clinical status and sex was not a significant factor in the model $\left(\mathrm{F}_{(3,65)}=1.13, \mathrm{p}=0.35\right)$. As noted above, age was entered as a covariate in the analysis and was not significant $\left(\mathrm{F}_{(3,65)}=1.51, \mathrm{p}=0.22\right)$. Similarly, when examining the effect of age and gender on individual variables, no significant effects were found. Univariate analyses (Analysis of Covariance -ANCOVA), with age as the covariate, also indicated no significant differences between the groups in relation to each of the dependent variables (Appendix 4.11).

However, when the effect of age was not controlled, Multivariate Analysis of Variance (MANOVA) revealed significant inter-group differences in relation to BMHSS 'Ageism' $\left(\mathrm{F}_{(1,72)}=5.47, \mathrm{p}=0.02 ; \mathrm{ES}=-0.56\right.$; observed power $\left.=0.64\right)$ (Appendix 4.12). Examination of mean scores on the BMHSS 'Ageism' scale (Figure 7) indicate that group scores differed significantly in the predicted direction, with the non clinical group expressing more negative attitudes to mental illness in later life than the clinical group. However, these results should be interpreted cautiously as both multivariate and univariate analyses were underpowered. 


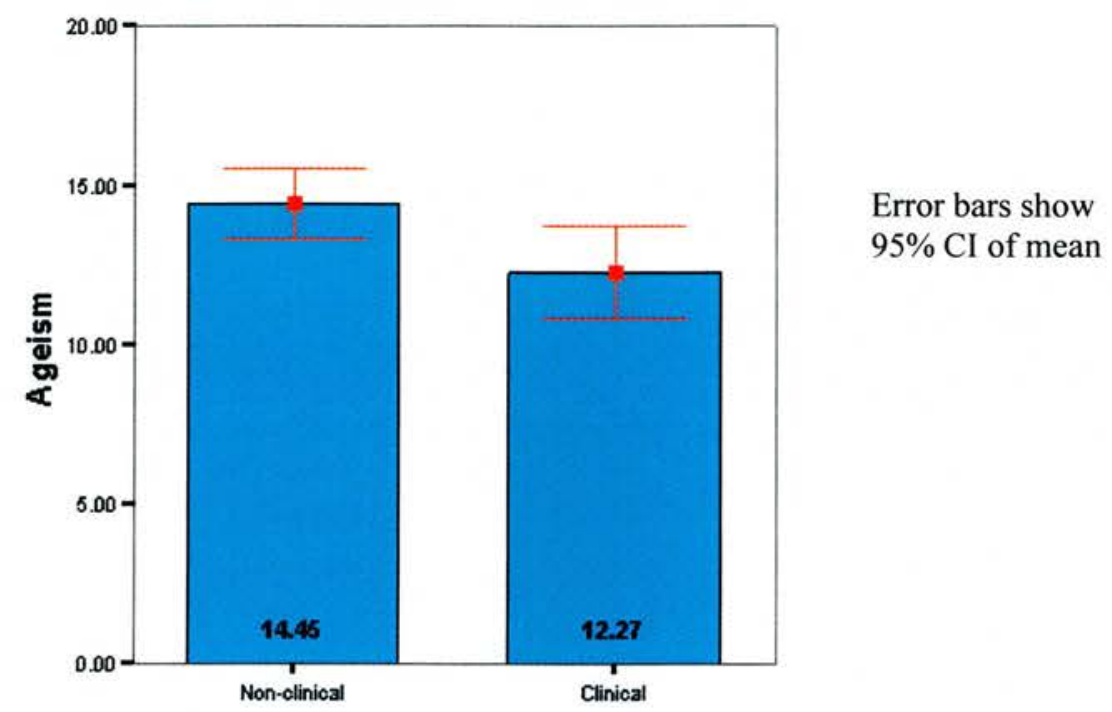

\section{Figure 7: Comparison of Group Mean Scores on BMHSS 'Ageism' Sub-scale.}

\subsection{Comparison of Attitudes to Mental Illness in Later Life in Those With and Without Prior Experience}

Independent samples $t$-tests were undertaken to compare mean scores on each of the dependent variables for participants with $(n=33)$ and without $(n=34)$ prior experience of mental illness across the whole sample. Table 12 indicates that, in line with Hypothesis 2, participants with prior experience of mental illness/mental health services endorsed more positive attitudes on the BMHSS 'Ageism' subscale ( ${ }_{(65)}=$ $2.55, \mathrm{p}=0.007)$. A review of the content of items included in this scale indicates that, on average, participants with prior experience of mental illness perceived age as less of a barrier to psychological treatment than participants without prior experience, suggesting a positive impact of experience on attitudes. As this was a predicted effect, one-tailed significance is reported. A medium to large effect size is noted, although power calculations indicate that 35 participants per group would be required to detect this effect (Clark-Carter, 2004). No significant differences in reported 
attitudes were found between those with and without past experience of mental illness/mental health services in relation to the two remaining dependent variables, although the poor psychometric properties of both BMHSS 'Stigma', and Understandability scales should be taken into account when interpreting this result. In addition, two-way analyses of variance revealed no significant interaction effects of group (i.e. clinical status) and past experience in relation to any of the dependent variables.

In summary, some positive effects of current service utilisation and experience of mental illness on attitudes were found, although positive effects did not extend to all dependent variables thus providing only partial support for Hypothesis 2 .

Table 12: Comparison of Attitudes to Mental IIIness in Later Life in Those With and Without Prior Experience.

\begin{tabular}{|c|c|c|c|c|c|c|c|}
\hline Measure/Sub-scale & Group & Mean & $\begin{array}{l}\text { Std } \\
\text { Dev }\end{array}$ & d.f. & $\begin{array}{c}t^{\star} \\
\text { (Sig.) }\end{array}$ & $\begin{array}{l}\text { Mean diff } \\
(95 \% \mathrm{CI})\end{array}$ & $\begin{array}{c}\text { Effect } \\
\text { Size }^{+}+ \\
\text {Observed } \\
\text { Power }\end{array}$ \\
\hline \multirow[t]{2}{*}{ BMHSS: 'Ageism' } & $\begin{array}{l}\text { With prior } \\
\text { experience }(N=33)\end{array}$ & 12.50 & 3.27 & \multirow[t]{2}{*}{65} & \multirow{2}{*}{$\begin{array}{c}2.55 \\
(p=0.007)\end{array}$} & \multirow{2}{*}{$\begin{array}{c}-2.40 \\
(-4.28 \text { to }-0.52)\end{array}$} & \multirow{2}{*}{$\begin{aligned} d & =0.60 \\
\mathrm{P} & =0.71\end{aligned}$} \\
\hline & $\begin{array}{l}\text { Without prior } \\
\text { experience }(\mathrm{N}=34)\end{array}$ & 14.90 & 4.36 & & & & \\
\hline \multirow[t]{2}{*}{ BMHSS: 'Stigma' } & $\begin{array}{l}\text { With prior } \\
\text { experience }(\mathrm{N}=33)\end{array}$ & 9.38 & 2.58 & \multirow[t]{2}{*}{65} & \multirow{2}{*}{$\begin{array}{c}0.89 \\
(p=0.20)\end{array}$} & \multirow{2}{*}{$\begin{array}{c}-0.51 \\
(-1.66 \text { to }-0.64)\end{array}$} & \multirow{2}{*}{$\begin{array}{l}d=0.20 \\
P=0.14\end{array}$} \\
\hline & $\begin{array}{l}\text { Without prior } \\
\text { experience }(\mathrm{N}=34)\end{array}$ & 9.89 & 2.12 & & & & \\
\hline \multirow[t]{2}{*}{ Understandability } & $\begin{array}{l}\text { With prior } \\
\text { experience }(\mathrm{N}=33)\end{array}$ & 8.85 & 3.95 & \multirow[t]{2}{*}{65} & \multirow{2}{*}{$\begin{array}{c}0.40 \\
(p=0.34)\end{array}$} & \multirow{2}{*}{$\begin{array}{c}-0.36 \\
(-2.12 \text { to } 1.41)\end{array}$} & \multirow{2}{*}{$\begin{array}{c}d=0.10 \\
\mathrm{P}=0.07\end{array}$} \\
\hline & $\begin{array}{l}\text { Without prior } \\
\text { experience }(N=34)\end{array}$ & 9.20 & 3.24 & & & & \\
\hline
\end{tabular}

* Equal variances assumed (i.e. Levene's test non-significant). Significance is one-tailed.

${ }^{+}$Calculated according to Cohen's d formula (see Clark-Carter, 2004, p. 181). 
3.2.3.3 Hypothesis 3: Negative attitudes to ageing will be associated with negative attitudes to mental illness in later life. Attitudes to ageing will vary significantly by clinical status and age (i.e. clinical participants and the oldest-old will endorse more negative attitudes).

\subsection{Between-group Analyses: Attitudes to Ageing}

Potential differences between clinical and non-clinical groups in terms of attitudes to ageing (i.e. as measured by responses to the AAQ (composite and sub-scales), and the RAME) were assessed using MANCOVA, with age as the covariate. Results from multivariate analyses indicate that participant status (i.e. clinical or nonclinical) was a significant factor in the model $\left(\mathrm{F}_{(4,64)}=4.38, \mathrm{p}=0.003\right)$. No significant effects of sex, or the interaction between clinical status and sex, were found. In contrast, age did achieve significance in the overall model $\left(\mathrm{F}_{(4,64)}=2.77, \mathrm{p}\right.$ $=0.03$ ). When examining the effect of age on individual variables, age was significant for the RAME (i.e. measure of internalised ageism $\left(\mathrm{F}_{(1,67)}=5.14, \mathrm{p}=\right.$ 0.03; Appendix 4.13). Univariate tests (ANCOVA, with age as the covariate) also revealed significant group differences in relation to three of the five dependent variables: AAQ Composite score $\left(\mathrm{F}_{(1,67)}=9.60, \mathrm{p}=0.003\right)$, AAQ 'Physical' subscale score $\left(\mathrm{F}_{(1,67)}=11.51, \mathrm{p}=0.001\right)$, and RAME score $\left(\mathrm{F}_{(1,67)}=15.75, \mathrm{p}<0.001\right)$.

Results from MANOVA shown in Table 13, indicate that when the effect of age was not statistically controlled, inter-group differences remained in relation to AAQ Composite score $\left(\mathrm{F}_{(1,72)}=15.67, \mathrm{p}<0.001\right)$, AAQ 'Physical' subscale score $\left(\mathrm{F}_{(1,72)}=\right.$ 21.34, $\mathrm{p}<0.001)$ and RAME score $\left(\mathrm{F}_{(1,72)}=11.28, \mathrm{p}=0.001\right)$. In addition, groups also differed significantly in relation to AAQ: Growth $\left(\mathrm{F}_{(1,72)}=7.49, \mathrm{p}=0.008\right.$; see 
Table 13 and Appendix 4.14). Boxplots of each of the main dependent (attitudes to ageing) variables are presented in Appendix 4.15. Table 13: Comparison of Attitudes to Ageing across Groups
(Age Not Controlled)

\begin{tabular}{|c|c|c|c|c|c|c|c|}
\hline Measure/Sub-scale & Group & Mean* & $\begin{array}{l}\text { Std } \\
\text { Dev }\end{array}$ & $95 \% \mathrm{CI}$ & d.f. & $\begin{array}{c}\text { F } \\
\text { (Sig.) }\end{array}$ & $\begin{array}{l}\text { Effect } \\
\text { Size }^{+} \text {\& } \\
\text { Observed } \\
\text { Power }\end{array}$ \\
\hline \multirow[t]{2}{*}{ AAQ: Composite } & $\begin{array}{l}\text { Non-clinical } \\
(\mathrm{N}=50)\end{array}$ & 57.44 & 10.08 & $54.58-60.30$ & \multirow[t]{2}{*}{1,72} & \multirow{2}{*}{$\begin{array}{c}15.67 \\
(p<0.001)\end{array}$} & \multirow{2}{*}{$\begin{array}{l}d=0.90 \\
\mathrm{P}=0.97\end{array}$} \\
\hline & $\begin{array}{l}\text { Clinical } \\
\text { Group }(\mathrm{N}=24)\end{array}$ & 67.42 & 10.30 & $63.29-71.55$ & & & \\
\hline \multirow[t]{2}{*}{ AAQ: 'Growth' } & $\begin{array}{l}\text { Non-clinical } \\
(\mathrm{N}=50)\end{array}$ & 29.17 & 3.87 & $27.95-30.39$ & \multirow[t]{2}{*}{1,72} & \multirow{2}{*}{$\begin{array}{c}7.49 \\
(p=0.008)\end{array}$} & \multirow{2}{*}{$\begin{array}{l}d=-0.65 \\
\mathrm{P}=0.77\end{array}$} \\
\hline & $\begin{array}{l}\text { Clinical } \\
\text { Group }(N=24)\end{array}$ & 26.22 & 5.16 & $24.47-27.99$ & & & \\
\hline \multirow[t]{2}{*}{ AAQ: 'Loss' } & $\begin{array}{l}\text { Non-clinical } \\
(\mathrm{N}=\mathbf{5 0})\end{array}$ & 17.22 & 6.20 & $15.60-18.84$ & \multirow[t]{2}{*}{1,72} & \multirow{2}{*}{$\begin{array}{c}2.26 \\
(p=0.14)\end{array}$} & \multirow{2}{*}{$\begin{array}{l}d=0.37 \\
\mathrm{P}=0.38\end{array}$} \\
\hline & $\begin{array}{l}\text { Clinical } \\
\text { Group }(\mathrm{N}=\mathbf{2 4})\end{array}$ & 19.37 & 4.62 & $17.03-21.70$ & & & \\
\hline \multirow[t]{2}{*}{ AAQ: 'Physical' } & $\begin{array}{l}\text { Non-clinical } \\
(\mathrm{N}=\mathbf{5 0})\end{array}$ & 26.62 & 4.19 & $25.41-27.82$ & \multirow[t]{2}{*}{1,72} & \multirow{2}{*}{$\begin{array}{c}21.34 \\
(\mathrm{p}<0.001)\end{array}$} & \multirow{2}{*}{$\begin{array}{l}\mathrm{d}=-1.01 \\
\mathrm{P}=0.99\end{array}$} \\
\hline & $\begin{array}{l}\text { Clinical } \\
\text { Group }(\mathrm{N}=\mathbf{2 4})\end{array}$ & 21.72 & 4.43 & $19.98-23.46$ & & & \\
\hline \multirow[t]{2}{*}{ RAME } & $\begin{array}{l}\text { Non-clinical } \\
(\mathrm{N}=\mathbf{5 0})\end{array}$ & 26.50 & 7.55 & $24.61-28.38$ & \multirow[t]{2}{*}{1,72} & \multirow{2}{*}{$\begin{array}{c}11.28 \\
(p=0.001)\end{array}$} & \multirow{2}{*}{$\begin{array}{l}d=0.78 \\
\mathrm{P}=0.91\end{array}$} \\
\hline & $\begin{array}{l}\text { Clinical } \\
\text { Group }(N=24)\end{array}$ & 32.07 & 4.31 & $29.36-34.80$ & & & \\
\hline
\end{tabular}

* Mean score after missing values replaced.

${ }^{+}$Calculated according to Cohen's d formula (see Clark-Carter, 2004, p. 181). Partial eta-squared values are provided in Appendix 4.14.

Effect sizes were in the medium to large range, with observed power achieved for four of the five variables (AAQ Composite, AAQ 'Growth', AAQ 'Physical', and RAME). The results indicate that the clinical group endorsed more negative attitudes to ageing than non-clinical group (e.g. AAQ Composite: $F_{(1,72)}=15.67, p<0.001$ ). Specifically, participants in the clinical group were less likely to endorse attitudes relating to 'Growth' (as ascertained by responses to such items as: 'As people get older they are better able to cope with life'; 'Wisdom comes with age') and 'Physical' factors of ageing (e.g. 'It is important to take exercise at any age'; 'I don't 
feel old'; $\left.\mathrm{F}_{(1,72)}=21.34, \mathrm{p}<0.001\right)^{12}$. Consistent with these findings, scores for internalised ageism (RAME) were also higher for clinical participants $\left(F_{(1,72)}=11.28\right.$, $\mathrm{p}=0.001$ ). No significant inter-group differences were observed in relation to the AAQ sub-scale of 'Loss'.

Interestingly, and in contrast to predictions, attitudes to ageing were not significantly more negative in the oldest-old. Across the entire sample, significant differences between the age cohorts were found only in relation to the AAQ 'Physical' sub-scale $\left(t_{(70)}=2.78, \mathrm{p}=0.004\right.$, one-tailed), with young-old participants (i.e. those aged between 65-79) expressing less positive views about the physical aspects of growing older (Appendix 4.16). The effect size between the groups was medium to large $(d=$ $0.62)$, and observed power was high $(\mathrm{P}=0.78)$.

\subsection{Association Between Attitudes to Ageing and Attitudes to Mental Illness in Later Life}

Correlations were undertaken to establish whether negative attitudes to ageing were significantly associated with negative attitudes to mental illness in later life across the whole sample, in accordance with predictions. Initially, correlations between participant age and each of the main dependent variables were undertaken to assess whether it was necessary to control for age in further analyses. Zero-order correlations were performed (i.e. bivariate correlations without controlling for other variables), followed by first-order partial correlations (holding age constant). Appendix 4.17 indicates that participant age was positively correlated with three of the main study measures: BMHSS 'Ageism' $(r=.27, \mathrm{p}=0.01)$, AAQ 'Growth' ( $r=$

${ }^{12}$ i.e. lower sub-scale scores indicate increased negativity. 
$.22, \mathrm{p}=0.03$ ), and AAQ 'Physical' $(r=.32, \mathrm{p}=0.003)$, thus accounting for 7.3 per cent, 4.8 per cent, and 10.2 per cent of the variance, respectively (i.e. $\mathrm{R}^{2}=$ correlation coefficient squared e.g. $0.27^{2}$ ). Given that at least some of the variance in the measures was accounted for by age, partial correlations were conducted in order to provide a purer indication of the relationship between attitudes to ageing and attitudes to mental illness in later life. All correlations were one-tailed, given the directional predictions of the hypothesis. Strength of correlations was assessed in relation to Cohen's (1988) estimate of $r= \pm .10-.29$ equating to small, $r= \pm .30-$ .49 equating to medium, and $\mathrm{r}= \pm .50-1.0$ representing a large correlation.

Appendix 4.17 indicates that significant inter-correlations were found between subscales of the main variables (e.g. between 'AAQ 'Growth', 'Loss', 'Physical') and between scales measuring similar constructs (e.g. between AAQ Composite score and RAME score). While the latter finding could be construed as evidence of convergent validity, it also raises the issue of possible confounding across scales which should be taken into account when considering the noted relationships between variables outlined below.

In line with the hypothesis, significant associations were found between negative attitudes to ageing (as measured by the AAQ), particularly attitudes relating to a lossdeficit view of ageing, and negative attitudes to mental illness, particularly those attitudes reflecting the perceived stigma of mental illness (Table 14). Scatterplots of (zero order) correlations are presented in Figure 8. As indicated in Table 14, the strongest associations were found between AAQ 'Loss' (i.e. the perception of old age as a time of loss, decline and loneliness) and Understandability (i.e. the view that 
depression is an inevitable part of old age given the losses encountered), with sufficient power to detect a medium effect size. An additional partial correlation which approached significance (not shown), was found between AAQ 'Growth' and BMHSS 'Stigma' $(r=-.20, \mathrm{P}=0.051)$, again in the predicted direction. An unexpected positive correlation was found between the AAQ 'Physical' sub-scale and BMHSS 'Ageism' $(r=.27, \mathrm{p}=0.01)$, although age accounted for over half of the variance (Table 14). Thus a correlation was found between positive attitudes to physical health in ageing and a negative view of mental illness in later life.

\section{Table 14: Correlations Between Attitudinal Variables}

\begin{tabular}{|c|c|c|c|c|}
\hline Variable(s) & $\begin{array}{c}\text { Correlation } \\
\text { Coefficient: } \\
\text { Zero-Order } \\
(\mathbf{N}=\mathbf{7 0})\end{array}$ & $\begin{array}{c}95 \% \\
\text { CI's for } \\
r^{*}\end{array}$ & $\begin{array}{c}\text { Correlation } \\
\text { Coefficient: } \\
\text { Partial }^{+} \\
(\mathrm{N}=69)\end{array}$ & $\begin{array}{l}95 \% \text { CI's } \\
\text { for } r\end{array}$ \\
\hline $\begin{array}{l}\text { AAQ composite score: } \\
\text { BMHSS 'Stigma' }\end{array}$ & & & $\begin{array}{c}r=.20 \\
(\mathrm{p}<0.05)\end{array}$ & 0.04 to 0.43 \\
\hline $\begin{array}{l}\text { AAQ 'Loss': } \\
\text { BMHSS 'Stigma' }\end{array}$ & $\begin{aligned} r & =.20 \\
(\mathrm{p} & <0.05)\end{aligned}$ & 0.04 to 0.43 & & \\
\hline $\begin{array}{l}\text { AAQ ‘Loss': } \\
\text { Understandability }\end{array}$ & $\begin{array}{c}r=.42 \\
(p<0.001)\end{array}$ & 0.20 to 0.59 & $\begin{array}{c}r=.41 \\
(\mathrm{p}<0.001)\end{array}$ & 0.19 to 0.59 \\
\hline $\begin{array}{l}\text { AAQ 'Physical': } \\
\text { BMHSS 'Ageism' }\end{array}$ & $\begin{array}{c}r=.27 \\
(\mathrm{p}=0.01)\end{array}$ & 0.04 to 0.47 & $\begin{array}{c}r=.20 \\
(\mathrm{p}<0.05)\end{array}$ & 0.04 to 0.43 \\
\hline
\end{tabular}

${ }^{*}$ Calculated according to formula in Clark-Carter (2004; p.505)

${ }^{+}$Age partialled out. 


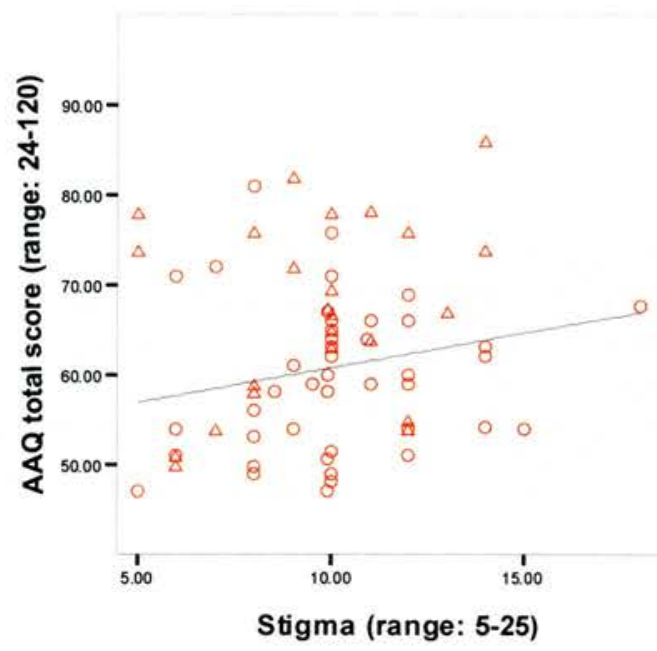

Group ID

O-Non-clinical Clinical

$$
\mathrm{Rsq}=0.03
$$

Group ID

O Non-clinical

$\triangle \quad$ Clinical

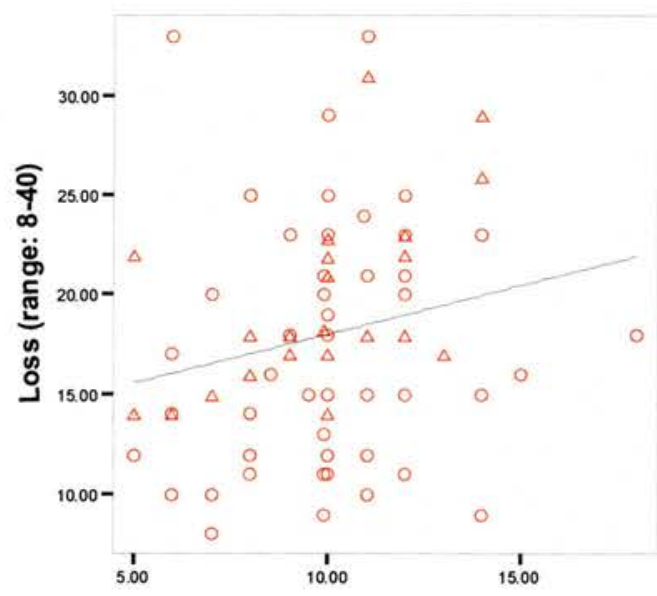

$$
\text { Rsq }=0.05
$$

Group ID

$\triangle \quad$ Non-clinical

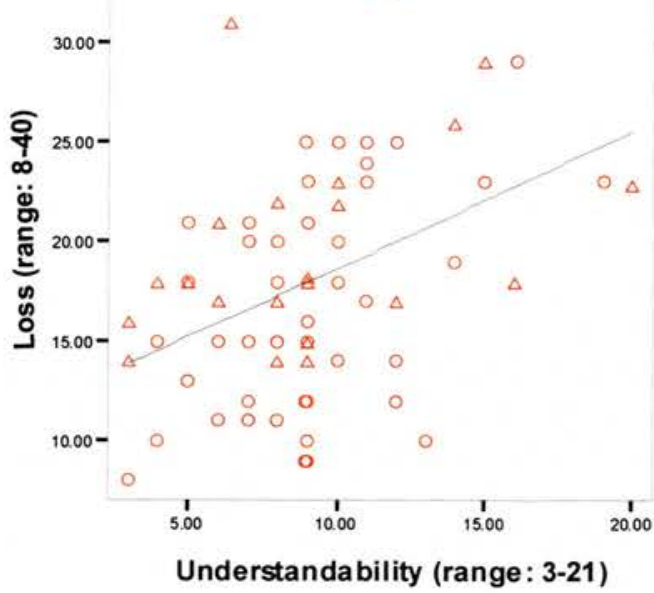

Rsq $=0.17$

Figure 8: Scatterplots of Correlations of Attitudinal Variables 
To explore this finding in more detail, a standard (simultaneous) multiple regression analysis was undertaken with BMHSS 'Ageism' (i.e. reflecting negative (ageist) views toward mental illness in later life) as the dependent variable (DV). Independent variables (IVs) were selected on the basis of the correlational analysis outlined above (see Appendix 4.17) and the hypotheses, and included age, gender, health status, past experience of mental illness, attitudes to ageing subscales, and understandability. The regression correlation matrix (Appendix 4.18) revealed several significant inter-correlations between the IVs raising the possibility of multicollinearity within the model. However, since inter-correlations were below 0.8 (i.e. the threshold proposed by Clark-Carter, 2004), and Variance Inflation Factor (VIF) and Tolerance statistics were within acceptable limits (i.e. VIF $<10.0$, Tolerance $>$ 0.1), multi-collinearity was not considered a significant problem in the model. Further diagnostic checks indicated that the assumptions of the regression model were met (i.e. independent residuals, linearity and homoscedasticity; see Appendix $4.18)^{13}$.

Summary statistics for the final regression model are presented in Table 15a which shows R Square (i.e. the amount of variation in the outcome variable accounted for by the model), Adjusted R Square (i.e. the estimate of $\mathrm{R}^{2}$ in the population which takes into account sample size and number of IVs); R Square Change (i.e. the increase in R Square attributable to the addition of the next independent variable in the equation); F Change and Significance of F Change (i.e. which provides the

\footnotetext{
${ }^{13}$ One potential outlier was detected with standardised residual values $> \pm 2.01$ (i.e. bigger than 3 SDs from the mean), however, once alpha was adjusted (by dividing 0.05 by the sample size), the standardised residual value (3.22) did not exceed the z-score for the corresponding (one tailed) level of significance (i.e. $\mathrm{z} \pm 3.39$ ). Thus, in line with recommendations by Clark-Carter (2004, p.332-333), the case was not removed from the analysis.
} 
significance of the change in $\mathrm{R}$ Square as a result of the addition of independent variables).

Table 15a: Regression Model Summary Statistics for BMHSS 'Ageism' as DV

\begin{tabular}{|l|c|c|c|c|c|c|}
\hline \multicolumn{1}{|c|}{$\begin{array}{c}\text { Model } \\
(\mathbf{N}=67)\end{array}$} & $\begin{array}{c}\text { R } \\
\text { Square }\end{array}$ & $\begin{array}{c}\text { Adjusted } \\
\text { R Square }\end{array}$ & $\begin{array}{c}\text { Std Error } \\
\text { of the } \\
\text { Estimate }\end{array}$ & $\begin{array}{c}\text { R Square } \\
\text { Change }\end{array}$ & $\begin{array}{c}\text { F } \\
\text { Change }\end{array}$ & Sig. \\
\hline $\begin{array}{l}1- \\
\text { Past Experience } \\
\text { Understandability }\end{array}$ & .164 & .138 & 3.73 & .164 & 6.293 & $\mathrm{p}=0.003$ \\
\hline
\end{tabular}

For each significant predictor variable, Table $15 \mathrm{~b}$ provides details of unstandardised and standardised beta coefficients, $t$ values and significance. From an initial model of eight predictors, non-significant variables were removed sequentially until only those variables contributing significantly to the model remained. Two IVs ('Past Experience' and 'Understandability') contributed significantly to the model, $\mathrm{R}^{2}=.16$ (Adjusted R Square $=.14$ ). Thus, predictors accounted for 16.4 per cent (Adjusted R Square, 13.8 per cent) of the total variance in BMHSS 'Ageism' $\left(\mathrm{F}_{(2,64)}=6.29, \mathrm{p}=\right.$ 0.003). The analysis was sufficiently powered to detect a medium effect size ${ }^{14}$ (i.e. a sample size of $50+8 k$, where $k$ is the number of predictors; Green, 1991) for the overall fit of the model. However, the analysis lacked power to test the contribution of individual predictors (a sample of $104+k$ is recommended, where $k$ is the number of predictors; Green, 1991), and overall sample size was insufficient to perform model validation (e.g. by data-splitting). A review of the correlation matrix (Appendix 4.18) indicates that the variable 'Past Experience (of mental illness)' was negatively correlated with both BMHSS 'Ageism' $(\mathrm{r}=-.31, \mathrm{p}=0.005)$, and AAQ

\footnotetext{
${ }^{14}$ Cohen's (1988) criteria indicate an $\mathrm{R}^{2}$ of approximately .02 as a small effect size, .13 as a medium effect size, and .26 as a large effect size.
} 
'Physical' $(\mathrm{r}=-.32, \mathrm{p}=0.004)$, raising questions for further research of whether personal experience of illness (and presumably also of contact with services) may underlie the association between attitudes to physical health (in ageing) and mental illness in later life reported earlier (Section 3.2.3.3.2). Alternative explanations for the latter result are explored in Section 4.2.3.

Table 15b: Regression Coefficients for BMHSS 'Ageism' as DV

\begin{tabular}{|l|r|r|c|r|r|r|r|r|r|}
\cline { 2 - 10 } \multicolumn{1}{c|}{} & \multicolumn{2}{c|}{$\begin{array}{c}\text { Unstandard. } \\
\text { Coefficients }\end{array}$} & $\begin{array}{c}\text { Standard. } \\
\text { Coefficients }\end{array}$ & \multicolumn{1}{c|}{$\boldsymbol{t}$} & \multicolumn{1}{c|}{ Sig. } & \multicolumn{2}{c|}{$\begin{array}{c}\text { 95\% Confidence } \\
\text { Interval } \\
\text { for B }\end{array}$} & \multicolumn{2}{c|}{$\begin{array}{c}\text { Collinearity } \\
\text { Statistics }\end{array}$} \\
\hline Model & \multicolumn{1}{|c|}{ B } & $\begin{array}{c}\text { Std. } \\
\text { Error }\end{array}$ & Beta & & & $\begin{array}{c}\text { Lower } \\
\text { Bound }\end{array}$ & $\begin{array}{c}\text { Upper } \\
\text { Bound }\end{array}$ & Tol. & VIF \\
\hline (Constant) & \multicolumn{1}{c|}{12.097} & 1.341 & & 9.018 & .000 & 9.417 & 14.777 & & \\
\hline Past Exp & -2.293 & .912 & -.288 & -2.513 & .014 & -4.115 & -.470 & .998 & 1.003 \\
\hline Understand. & .304 & .128 & .272 & 2.375 & .021 & .048 & .560 & .998 & 1.003 \\
\hline
\end{tabular}

3.2.3.4 Hypothesis 4: Reported engagement in health-related behaviours and subjective well-being will be lower in the clinical sample. Negative attitudes to ageing and to mental illness will be associated with lower endorsement of health-related behaviours and poorer subjective well-being across the entire sample.

\subsection{Between-group Analyses: Reported Health Behaviours Checklist (RHBC) and $\mathrm{GHQ}-12$}

A between-groups MANCOVA, with age as the covariate, was conducted to establish whether scores on variables assessing health behaviours and subjective well-being (Reported Health Behaviours Checklist - RHBC; General Health Questionnaire - GHQ-12) differed significantly according to clinical status, with lower scores on all variables predicted in the clinical group. Multivariate tests indicated that participant status (i.e. clinical or non-clinical) was a significant factor in the model $\left(\mathrm{F}_{(3,65)}=9.26, \mathrm{p}<0.001\right)$. Sex was entered as a between-participants 
fixed factor and was not significant $\left(\mathrm{F}_{(3,65)}=0.21, \mathrm{p}=0.90\right)$. In addition, the interaction between clinical status and sex was not a significant factor in the model $\left(F_{(3,65)}=0.58, p=0.63\right)$. Age was entered as a covariate in the analysis and was not significant $\left(F_{(3,65)}=0.15, p=0.93\right)$. Similarly, when examining the effect of age and gender on individual variables, no significant effects were found (Appendix 4.19). Univariate analyses (ANCOVA, with age as the covariate) revealed significant differences between the groups in relation to RHBC total score $\left(F_{(1,67)}=11.07, p=\right.$ $0.001)$, RHBC 'Cognitive Affective' subscale $\left(\mathrm{F}_{(1,67)}=17.51, \mathrm{p}<0.001\right)$, and GHQ$12\left(\mathrm{~F}_{(1,67)}=13.90, \mathrm{p}<0.001\right)$.

As indicated in Table 16, when age was not controlled, MANOVA revealed significant inter-group differences in relation to all four dependent variables (see also Appendix 4.20). Examination of mean scores on all variables indicate that group scores differed significantly in the predicted direction, with the clinical group reporting less engagement in health-related behaviours, fewer cognitive and affective regulation strategies, and lower subjective well-being (Appendix 4.21). Effect sizes were in the medium to large range, with observed power achieved for three of the four variables (RHBC total score; RHBC 'Cognitive Affective' subscale; and GHQ12). The largest inter-group difference was observed in relation to the RHBC 'Cognitive Affective' subscale $\left(\mathrm{F}_{(1,72)}=26.93, \mathrm{p}<0.001\right)$, indicating that clinical participants reported engagement in fewer cognitive-emotional regulation strategies than non-clinical participants. 
Table 16: Comparison of Group Scores on RHBC and GHQ-12 (Age Not Controlled)

\begin{tabular}{|c|c|c|c|c|c|c|c|}
\hline Measure/Sub-scale & Group & Mean* & $\begin{array}{l}\text { Std } \\
\text { Dev }\end{array}$ & $95 \%$ CI & d.f. & $\begin{array}{c}\text { F } \\
\text { (Sig.) }\end{array}$ & $\begin{array}{l}\text { Effect } \\
\text { Size }^{+} \& \\
\text { Observed } \\
\text { Power }\end{array}$ \\
\hline \multirow[t]{2}{*}{ RHBC total score } & $\begin{array}{l}\text { Non-clinical } \\
(\mathrm{N}=50)\end{array}$ & 72.62 & 8.90 & $70.21-75.25$ & \multirow{2}{*}{1,72} & \multirow{2}{*}{$\begin{array}{c}15.56 \\
(\mathbf{p}<\mathbf{0 . 0 0 1 )}\end{array}$} & \multirow{2}{*}{$\begin{array}{l}d=-0.88 \\
\mathrm{P}=0.97\end{array}$} \\
\hline & $\begin{array}{l}\text { Clinical } \\
\text { Group }(\mathrm{N}=\mathbf{2 4})\end{array}$ & 63.96 & 8.90 & $60.32-67.60$ & & & \\
\hline \multirow[t]{2}{*}{$\begin{array}{l}\text { RHBC: } \\
\text { 'Health Action' }\end{array}$} & $\begin{array}{l}\text { Non-clinical } \\
(\mathrm{N}=50)\end{array}$ & 47.37 & 7.63 & $45.22-49.22$ & \multirow[t]{2}{*}{1,72} & \multirow{2}{*}{$\begin{array}{c}4.52 \\
(p=0.04)\end{array}$} & \multirow{2}{*}{$\begin{array}{l}d=-0.52 \\
P=0.55\end{array}$} \\
\hline & $\begin{array}{l}\text { Clinical } \\
\text { Group }(\mathrm{N}=\mathbf{2 4})\end{array}$ & 43.34 & 7.65 & $40.24-46.45$ & & & \\
\hline \multirow{2}{*}{$\begin{array}{l}\text { RHBC: } \\
\text { 'Cognitive- } \\
\text { Affective' }\end{array}$} & $\begin{array}{l}\text { Non-clinical } \\
(\mathrm{N}=50)\end{array}$ & 25.36 & 3.10 & $24.32-26.39$ & \multirow[t]{2}{*}{1,72} & \multirow{2}{*}{$\begin{array}{c}26.93 \\
(\mathrm{p}<0.001)\end{array}$} & \multirow{2}{*}{$\begin{array}{l}d=-1.11 \\
\mathrm{P}=0.99\end{array}$} \\
\hline & $\begin{array}{l}\text { Clinical } \\
\text { Group }(\mathrm{N}=24)\end{array}$ & 20.62 & 4.67 & $19.12-22.11$ & & & \\
\hline \multirow[t]{2}{*}{ GHQ-12 } & $\begin{array}{l}\text { Non-clinical } \\
(\mathrm{N}=\mathbf{5 0})\end{array}$ & 10.39 & 5.40 & $8.76-12.01$ & \multirow[t]{2}{*}{1,72} & \multirow{2}{*}{$\begin{array}{c}17.62 \\
(\mathbf{p}<\mathbf{0 . 0 0 1 )}\end{array}$} & \multirow{2}{*}{$\begin{array}{l}d=0.94 \\
\mathrm{P}=0.99\end{array}$} \\
\hline & $\begin{array}{l}\text { Clinical } \\
\text { Group }(\mathrm{N}=\mathbf{2 4})\end{array}$ & 16.39 & 6.45 & $14.04-18.73$ & & & \\
\hline
\end{tabular}

* Mean score after missing values replaced.

${ }^{+}$Calculated according to Cohen's d formula (see Clark-Carter, 2004, p. 181). Partial eta-squared values are provided in Appendix 4.20.

\subsection{Association between Health Behaviours, Subjective Well-being and Attitudes}

Correlations were performed to establish whether negative attitudes to ageing and to mental illness were significantly associated with poorer engagement in health-related behaviours and lower subjective well-being across the entire sample, in accordance with predictions. Given that previous analyses indicated age was positively correlated with several of the main study measures, zero-order correlations were performed (i.e. bivariate correlations without controlling for other variables), followed by first-order partial correlations (holding age constant) in order to provide a purer indication of the relationship between attitudes, behaviours and subjective well-being (Appendix 4.22). All correlations were one-tailed, given the directional predictions of the hypothesis. Strength of correlations was assessed in accordance with Cohen's (1988) criteria (see Section 3.2.3.3.2). 
As indicated in Table 17, significant correlations were found between attitudes to ageing and both the RBHC (total and subscales) and GHQ-12 in the predicted direction. There was sufficient power to detect medium to large effect sizes (i.e. ES $>$.30). Thus, participants who endorsed more negative attitudes to ageing and expressed attitudes consistent with internalised ageism, also reported less healthrelated behaviours, and lower subjective wellbeing (e.g. Rame: GHQ-12, r = .69, p < 0.001). Conversely, participants who endorsed more positive attitudes to ageing (as assessed by AAQ subscale scores 'Growth' and 'Physical') also reported engagement in healthy behaviours (e.g. AAQ 'Physical': RHBC 'CognitiveAffective' subscales, $\mathrm{r}=.38, \mathrm{p}=0.001$ ), and higher subjective wellbeing (e.g. AAQ 'Growth: GHQ-12, $\mathrm{r}=-.30, \mathrm{p}=0.01)$. Scatterplots of (selected) correlations are presented in Appendix 4.23.

Table 17: Partial Correlations between Reported Health Behaviours, Subjective Well-being and Attitudinal Variables

\begin{tabular}{|c|c|c|c|c|}
\hline & $\begin{array}{c}\text { RHBC total } \\
\text { score } \\
(\mathrm{N}=69)\end{array}$ & $\begin{array}{l}\text { Health } \\
\text { Action } \\
(\mathrm{N}=69)\end{array}$ & $\begin{array}{c}\text { Cognitive } \\
\text { Affective } \\
(\mathrm{N}=69)\end{array}$ & $\begin{array}{c}\text { GHQ-12 } \\
(\mathbf{N}=69)\end{array}$ \\
\hline GHQ-12 & $\begin{array}{c}r=-.30 \\
(\mathrm{p}=\mathbf{0 . 0 0 6})\end{array}$ & $\begin{array}{l}r=-.20 \\
(\mathrm{p}=\mathbf{0 . 0 5})\end{array}$ & $\begin{aligned} r & =-.31 \\
(p & =.004)\end{aligned}$ & \\
\hline BMHSS 'Ageism' & $\begin{aligned} r & =-.07 \\
(\mathrm{p} & =0.30)\end{aligned}$ & $\begin{array}{l}r=-.12 \\
(\mathrm{p}=0.16)\end{array}$ & $\begin{array}{c}r=.07 \\
(\mathrm{p}=0.26)\end{array}$ & $\begin{aligned} r & =-.12 \\
(\mathrm{p} & =0.17)\end{aligned}$ \\
\hline BMHSS 'Stigma' & $\begin{aligned} r & =-.08 \\
(\mathrm{p} & =0.27)\end{aligned}$ & $\begin{array}{l}r=-.04 \\
(\mathrm{p}=0.36)\end{array}$ & $\begin{aligned} r & =-.09 \\
(\mathrm{p} & =0.22)\end{aligned}$ & $\begin{aligned} r & =-.11 \\
(\mathrm{p} & =0.19)\end{aligned}$ \\
\hline Understandability & $\begin{array}{c}r=.09 \\
(p=0.23)\end{array}$ & $\begin{array}{l}r=.13 \\
(p=0.14)\end{array}$ & $\begin{aligned} r & =-.03 \\
(p & =0.39)\end{aligned}$ & $\begin{array}{c}r=.18 \\
(\mathrm{p}=0.07)\end{array}$ \\
\hline AAQ Composite Score & $\begin{array}{c}r=-.37 \\
(\mathrm{p}=\mathbf{0 . 0 0 1 )}\end{array}$ & $\begin{array}{r}r=-.19 \\
(p=0.06)\end{array}$ & $\begin{array}{c}r=-.50 \\
(\mathrm{p}<0.001)\end{array}$ & $\begin{array}{c}r=.61 \\
(\mathrm{p}<0.001)\end{array}$ \\
\hline AAQ 'Growth' & $\begin{array}{c}r=.40 \\
(\mathrm{p}<\mathbf{0 . 0 0 1 )}\end{array}$ & $\begin{array}{c}r=.26 \\
(p=0.01)\end{array}$ & $\begin{array}{c}r=.42 \\
(\mathrm{p}<\mathbf{0 . 0 0 1 )}\end{array}$ & $\begin{aligned} r & =-.30 \\
(\mathrm{p} & =\mathbf{0 . 0 1})\end{aligned}$ \\
\hline AAQ 'Loss' & $\begin{aligned} r & =-.12 \\
(\mathrm{p} & =0.16)\end{aligned}$ & $\begin{array}{c}r=.03 \\
(\mathrm{p}=0.41)\end{array}$ & $\begin{array}{c}r=-.32 \\
(\mathrm{p}=\mathbf{0 . 0 0 3})\end{array}$ & $\begin{array}{c}r=.57 \\
(\mathrm{p}<\mathbf{0 . 0 0 1 )}\end{array}$ \\
\hline AAQ 'Physical' & $\begin{array}{c}r=.35 \\
(\mathrm{p}=\mathbf{0 . 0 0 1 )}\end{array}$ & $\begin{array}{c}r=.23 \\
(\mathrm{p}=\mathbf{0 . 0 3})\end{array}$ & $\begin{array}{c}r=.38 \\
(p<0.001)\end{array}$ & $\begin{array}{c}r=-.45 \\
(\mathrm{p}<\mathbf{0 . 0 0 1 )}\end{array}$ \\
\hline Rame & $\begin{aligned} r & =-.29 \\
(\mathrm{p} & =\mathbf{0 . 0 1})\end{aligned}$ & $\begin{aligned} r & =-.16 \\
(\mathrm{p} & =\mathbf{0 . 0 9})\end{aligned}$ & $\begin{array}{c}r=-.35 \\
(p=0.001)\end{array}$ & $\begin{array}{c}r=.69 \\
(\mathrm{p}<0.001)\end{array}$ \\
\hline
\end{tabular}


Contrary to predictions, no significant correlations were found between scores on measures of attitudes to mental illness and either the RHBC (total or sub-scales) or GHQ-12. A review of the correlation coefficients between attitudes to mental illness and both the RHBC and GHQ-12 indicates small effect sizes (i.e. $r=.10-.29$; Cohen, 1988), and retrospective power analysis indicates that between 600 and 700 participants would be needed to detect such an effect to achieve power of 0.8 and $\alpha=$ 0.05. Thus, on the basis of the current research, and given the noted psychometric problems (i.e. poor internal reliability) of at least two of the attitudes to mental illness measures, it would be prudent not to dismiss the possibility that negative attitudes to mental illness are also associated with poorer health behaviours and poorer psychological well-being.

Regression analyses were undertaken to ascertain whether attitudes to mental illness and ageing were predictive of reported engagement in health-related behaviours and subjective well-being. Separate standard (simultaneous) multiple regression analyses were undertaken with RHBC (total score) and GHQ-12 (total score) as dependent variables (DVs). Independent variables (IVs) were selected on the basis of the correlational analysis outlined above (see Table 17, and Appendix 4.22) and hypotheses, and included demographic variables, BMHSS subscales, Understandability, Attitudes to Ageing subscales, and Rame total score. Summary statistics and regression coefficients for the final regression models are presented below. Tolerance and VIF statistics for both models were within acceptable limits, and diagnostic checks indicated the assumptions of the models were met (Appendices $4.24 ; 4.25$ ). 
Table 18a indicates that $\mathrm{R}^{2}=.22$ (Adjusted $\mathrm{R}$ Square $=.20$ ). Thus, 22 per cent (Adjusted R Square $=20$ per cent) of the variance in RHBC total score was accounted for by two of the Attitudes to Ageing sub-scales (i.e. AAQ 'Growth' and AAQ 'Physical'), $\mathrm{F}_{(2,71)}=9.93, \mathrm{p}<0.001$, supporting the previously observed association between positive attitudes to ageing and reported engagement in proactive health-related behaviours (e.g. having regular medical check-ups, eating well, and avoiding harmful health habits). Attitudes to mental illness variables did not contribute significantly to the model (Table 18b). However, although there was sufficient power to detect a medium to large effect size (i.e. a sample size of $50+8 k$, where $k$ is the number of predictors; Green, 1991) for the overall fit of the model, there was insufficient power to test the contribution of individual predictors or the validity of the model (e.g. by data-splitting).

Table 18a: Regression Model Summary Statistics for RHBC Total Score as DV

\begin{tabular}{|c|c|c|c|c|c|c|}
\hline $\begin{array}{c}\text { Model } \\
(\mathbf{N}=\mathbf{7 2})\end{array}$ & $\begin{array}{c}\text { R } \\
\text { Square }\end{array}$ & $\begin{array}{c}\text { Adjusted } \\
\text { R Square }\end{array}$ & $\begin{array}{c}\text { Std Error } \\
\text { of the } \\
\text { Estimate }\end{array}$ & $\begin{array}{c}\text { R Square } \\
\text { Change }\end{array}$ & $\begin{array}{c}\text { F } \\
\text { Change }\end{array}$ & Sig. \\
\hline $\begin{array}{c}\text { 1- } \text { AAQ 'Growth' } \\
\text { AAQ 'Physical' }\end{array}$ & .22 & .20 & 8.78 & .22 & 9.93 & $\mathrm{p}<0.001$ \\
\hline
\end{tabular}

Table 18b: Regression Coefficients for RHBC Total Score as DV

\begin{tabular}{|l|r|r|r|r|r|r|r|r|r|}
\cline { 2 - 10 } \multicolumn{1}{c|}{} & \multicolumn{2}{c|}{$\begin{array}{c}\text { Unstandard. } \\
\text { Coefficients }\end{array}$} & \multicolumn{1}{c|}{$\begin{array}{c}\text { Standard. } \\
\text { Coefficients }\end{array}$} & \multicolumn{1}{c|}{$\boldsymbol{t}$} & \multicolumn{2}{c|}{ Sig. } & \multicolumn{2}{c|}{$\begin{array}{c}\text { 95\% Confidence } \\
\text { Interval for B }\end{array}$} & \multicolumn{2}{c|}{$\begin{array}{c}\text { Collinearity } \\
\text { Statistics }\end{array}$} \\
\hline Model & \multicolumn{1}{c|}{ B } & $\begin{array}{c}\text { Std. } \\
\text { Error }\end{array}$ & Beta & & & $\begin{array}{c}\text { Lower } \\
\text { Bound }\end{array}$ & $\begin{array}{c}\text { Upper } \\
\text { Bound }\end{array}$ & Tol. & VIF \\
\hline (Constant) & 40.485 & 6.779 & & 5.972 & .000 & 26.969 & 54.001 & & \\
\hline 'Growth' & .575 & .282 & .265 & 2.036 & .046 & .012 & 1.138 & .650 & 1.537 \\
\hline 'Physical' & .526 & .264 & .259 & 1.993 & .050 & .000 & 1.053 & .650 & 1.537 \\
\hline
\end{tabular}

Tables 19a and 19b present the results of the regression analysis with GHQ-12 as the dependent variable. Four IVs contributed significantly to the final model, $\mathrm{R}^{2}=.58$ 
(Adjusted R Square $=.55$ ), representing a large effect size. Thus, in addition to age, negative attitudes to mental illness (BMHSS 'Stigma') and to ageing (i.e. AAQ 'Loss', RAME) accounted for 58 per cent (Adjusted R Square $=55$ per cent) of the total variance in subjective well-being, $\mathrm{F}_{(4,67)}=22.99, \mathrm{p}<0.001$. A review of standardised coefficients (Table 19b) indicates that internalised ageism (RAME) is the best predictor of the DV, however, as for previous regression analyses, there was insufficient power to test the contribution of individual predictors in the model.

Table 19a: Regression Model Summary Statistics for GHQ-12 total score as DV

\begin{tabular}{|l|c|c|c|c|c|c|}
\hline \multicolumn{1}{|c|}{$\begin{array}{c}\text { Model } \\
(\mathbf{N}=\mathbf{7 4})\end{array}$} & $\begin{array}{c}\text { R } \\
\text { Square }\end{array}$ & $\begin{array}{c}\text { Adjusted } \\
\text { R Square }\end{array}$ & $\begin{array}{c}\text { Std Error } \\
\text { of the } \\
\text { Estimate }\end{array}$ & $\begin{array}{c}\text { R Square } \\
\text { Change }\end{array}$ & $\begin{array}{c}\text { F } \\
\text { Change } \\
\text { (Sig.) }\end{array}$ & Sig. \\
\hline $\begin{array}{l}\text { Age }- \\
\begin{array}{l}\text { BMHSS 'Stigma' } \\
\text { AAQ 'Loss' } \\
\text { Rame }\end{array}\end{array}$ & .58 & .55 & 4.31 & .58 & 22.99 & $\mathrm{p}<0.001$ \\
\hline
\end{tabular}

Table 19b: Regression Coefficients for GHQ-12 Total Score as DV

\begin{tabular}{|l|r|r|r|r|r|r|r|r|r|}
\cline { 2 - 11 } \multicolumn{1}{c|}{} & \multicolumn{2}{c|}{$\begin{array}{c}\text { Unstandard. } \\
\text { Coefficients }\end{array}$} & \multicolumn{1}{c|}{$\begin{array}{c}\text { Standard. } \\
\text { Coefficients }\end{array}$} & $\boldsymbol{t}$ & \multicolumn{2}{c|}{ Sig. } & \multicolumn{2}{c|}{$\begin{array}{c}\text { 95\% Confidence } \\
\text { Interval for B }\end{array}$} & \multicolumn{2}{c|}{$\begin{array}{c}\text { Collinearity } \\
\text { Statistics }\end{array}$} \\
\hline Model & \multicolumn{1}{c|}{ B } & $\begin{array}{c}\text { Std. } \\
\text { Error }\end{array}$ & Beta & & & $\begin{array}{c}\text { Lower } \\
\text { Bound }\end{array}$ & $\begin{array}{c}\text { Upper } \\
\text { Bound }\end{array}$ & Tol. & VIF \\
\hline (Constant) & 11.487 & 5.392 & & 2.130 & .037 & .725 & 22.249 & & \\
\hline Age & -.178 & .064 & -.222 & -2.762 & .007 & -.306 & -.049 & .978 & 1.023 \\
\hline 'Stigma' & -.474 & .207 & -.187 & -2.289 & .025 & -.888 & -.061 & .939 & 1.065 \\
\hline 'Loss' & .354 & .106 & .319 & 3.336 & .001 & .142 & .565 & .687 & 1.456 \\
\hline Rame & .458 & .084 & .514 & 5.471 & .000 & .291 & .625 & .713 & 1.402 \\
\hline
\end{tabular}




\subsubsection{SUMMARY OF RESULTS}

\subsubsection{Hypothesis-related Results}

Results relating to the main study hypotheses are summarised below. Results should be interpreted, where applicable, within the context of aforementioned statistical limitations (e.g. small sample size).

Hypothesis 1: Older people will endorse more negative attitudes to mental illness than younger people. Intra-cohort attitudes will differ by age and gender.

- There was no clear differentiation of attitudes between younger and older adults, with both groups expressing a mixture of positive and negative views of mental illness.

- There was no indication of enhanced negativity in the oldest-old, with the exception that participants in the oldest-old cohort expressed increased perceived difficulty in talking to people with mental health problems.

- There was no effect of clinical status in relation to general attitudes to mental illness.

- Men were generally not more negative in their views than women.

On the basis of these results, Hypothesis 1 was not supported. That is, in the current sample, older peoples' attitudes to mental illness did not differ significantly from those of younger people. Overall, attitudes did not vary significantly by age, gender or clinical status.

Hypothesis 2: Participants currently in receipt of mental health services will endorse more positive attitudes to mental illness than those not in receipt of services. Those with prior experience of mental illness/mental health services will endorse more positive attitudes than those without such experience. 
- When participant age was statistically controlled, no significant differences were found between clinical and non-clinical groups in relation to attitudes to mental illness in later life. However, when age was not controlled, participants in the non-clinical (i.e. older) group expressed more negative (ageist) attitudes to mental illness than participants in the clinical group.

- Participants with prior experience of mental illness endorsed more positive attitudes towards mental illness in later life, perceiving age as less of a barrier to treatment than participants without prior experience. No significant differences were found between those with and without past experience in relation to perception of stigma or understandability.

- No significant interaction effects were found between clinical status, experience, and attitudes.

Taken together, these results provide partial support for Hypothesis 2. Attitudes to mental illness in later life were more positive in those with personal experience of mental illness/mental health services. Understandability and perceived stigma of mental illness did not differ by experience.

Hypothesis 3: Negative attitudes to ageing will be associated with negative attitudes to mental illness in later life. Attitudes to ageing will vary significantly by clinical status and age (i.e. clinical participants and the oldest-old will endorse more negative attitudes).

- Negative attitudes to ageing were associated with negative attitudes to mental illness in later life. Specifically, attitudes to ageing indicative of a loss-deficit view of growing older were associated with stronger endorsement of understandability (i.e. expectations of depression in later life) and of the perceived stigma of mental illness.

- Positive attitudes to physical health in ageing were associated with negative attitudes to mental illness in later life. It was proposed that (lack of) personal experience of illness (and presumably therefore also of contact with services) may underlie this association. 
- Between-group analyses indicated that attitudes differed according to clinical status, irrespective of whether age was controlled. Thus, attitudes to ageing were significantly more negative in the clinical sample.

- There was no support for the prediction of enhanced negativity in the oldestold participants.

The results outlined above provide partial support for Hypothesis 3 . Negative attitudes to ageing were associated with negative attitudes to mental illness in later life, however, attitudes were not more negative in the oldest-old. Results suggest a potential positive mediating effect of experience on attitudes to mental illness.

Hypothesis 4: Reported engagement in health-related behaviours and subjective well-being will be lower in the clinical sample. Negative attitudes to ageing and to mental illness will be associated with lower endorsement of health-related behaviours and poorer subjective well-being across the entire sample.

- Participants in the clinical group reported significantly less engagement in health-related behaviours, and lower subjective well-being. The largest intergroup difference was observed in relation to cognitive-affective strategies indicating that clinical participants engaged in fewer cognitive-emotional regulation strategies than non-clinical participants.

- Across the entire sample, negative attitudes to ageing and higher internalised ageism were associated with poorer reported engagement in health-related behaviours, and lower subjective wellbeing. Positive attitudes to ageing were associated with stronger endorsement of healthy behaviours and higher subjective wellbeing.

- There were no significant correlations found between attitudes to mental illness and health behaviours or well-being.

- Positive attitudes to ageing, specifically attitudes related to personal growth and health, significantly predicted engagement in proactive health-related behaviours. 
- Negative attitudes to mental illness (i.e. perceived stigma of mental illness) and to ageing (i.e. perception of ageing as a time of loss; high internalised ageism) significantly predicted lower subjective well-being.

Hypothesis 4 is partially supported. Clinical participants endorsed fewer healthrelated behaviours and reported lower subjective well-being that non-clinical participants. Negative attitudes to ageing were associated with lower scores on both outcome variables, however, there was insufficient power to adequately assess the role of negative attitudes to mental illness.

\section{Between-group Results}

Results for between-group comparisons are summarised in Table 20. Implications of results for current and future research are considered in more detail in Chapter 4.

\section{Table 20: Summary of Between-group Comparisons}

\begin{tabular}{|l|l|}
\hline Non-Clinical Group & \multicolumn{1}{c|}{ Clinical Group } \\
\hline More negative attitudes to mental & $*$ More positive attitudes to mental \\
illness in later life & illness in later life \\
$*$ More positive attitudes to ageing & More negative attitudes to ageing \\
Higher reported subjective health & $*$ Poorer reported subjective health \\
More reported engagement in & $*$ Less reported engagement in \\
health behaviours & health behaviours \\
$*$ Higher subjective well-being & $*$ Lower subjective well-being \\
\hline
\end{tabular}




\section{4 - DISCUSSION}

\subsection{OVERVIEW AND SUMMARY OF STUDY FINDINGS}

This study explores older people's attitudes to mental illness, and considers whether attitudes are related to personal experience and/or are a reflection of broader underlying ageist misconceptions. Due to the paucity of research in this area, and particularly the relative neglect of the views of older people with and without mental health difficulties, a combined qualitative and quantitative methodology was used to explore and compare the views of service users and non-service users in relation to each of the above issues.

The qualitative analysis indicated that participants endorsed a broad range of positive and negative attitudes to mental illness, with more negative views expressed by those without prior experience of mental illness. Stigma and social exclusion were perceived by participants in both groups as very real consequences of being 'labelled' as mentally ill, despite indications of increasing societal acceptance of mental illness. Stigmatising societal views were considered to be perpetuated by a general lack of knowledge, awareness and understanding of mental illness given the relative 'invisibility' of conditions like depression. Negative stereotypes of mental illness appeared to reflect ambiguity about the cause, course and perceived controllability of different illnesses, reflected in non-clinical participants' beliefs regarding self-sufficiency (e.g. the ability to 'shake' oneself out of depression). At a broader level, ageism was perceived to impact negatively on health professionals' attitudes towards and treatment of older people with mental health difficulties, 
although this view was held primarily by non-clinical participants (i.e. those not currently attending services). Positive attitudes to mental illness were also permeated by ageist assumptions in the non-clinical group, with younger people perceived as more deserving of good mental health.

In line with results from qualitative analyses, survey results indicated that older people held a range of positive and negative views to mental illness, and that, contrary to expectation, the general views of older people were neither dissimilar to those of younger people, nor more negative in the oldest-old. While negative attitudes to mental illness were associated with negative attitudes to ageing across the entire sample, clinical participants (and those with prior experience of mental illness) reported more positive attitudes to mental illness, and more negative attitudes to ageing than non-clinical participants, for whom the reverse was true. This finding may reflect greater capacity on the part of non-clinical participants to approach ageing as a time of continual growth and maximisation of function (Depp \& Jeste, 2006), a view further supported by the observed association between positive attitudes to ageing and engagement in health-behaviours and higher subjective wellbeing. Of course, it is also the case that better psychological self-regulation in ageing may be easier to achieve if one has not yet encountered the potential challenges and losses associated with growing older, and may in fact be a protective factor against the onset of depression and other mental health disorders (Ebner et al., 2006). Similarly, one may not self-identify as being old if there is marked incongruence between personal experiences of ageing and negative social stereotypes (Degnen, 2007). These issues are considered further below. 


\subsection{FURTHER DISCUSSION OF STUDY FINDINGS}

\subsubsection{Hypothesis 1}

In contrast to research reporting that older people have negative attitudes to mental illness (e.g. Lundervold \& Young, 1992), the present study found that older people endorsed a range of positive and negative attitudes to mental illness, and held broadly similar views to younger adults (see Section 3.2.3.1.2). In terms of negative attitudes, as highlighted in studies by Connery and Davidson (2006), and Hayward and Bright (1997), almost half of both the younger and older adult samples compared in the current study indicated that they would not wish anyone to know if they were suffering from mental health problems, with men were more likely than women to endorse this view. In contrast to findings from other studies (e.g. Scottish Executive, 2004), only a small percentage of both younger and older participants perceived individuals with mental health problems as dangerous, however, more older people than younger people agreed with the statement that there was a need to better protect the public. Participants from the oldest-old age cohort also reported greater perceived difficulty talking to individuals with mental health problems compared to participants in the younger-old group. Additional analyses revealed that views did not differ markedly by clinical status, although, interestingly, the non-clinical group were less lenient in their views regarding the rights of those with mental health difficulties. One could speculate this might reflect an attempt on the part of nonclinical participants to distance themselves from a group they do not wish to be part of. 
The persistence of negative attitudes to mental illness across younger and older age groups is concerning. Several explanations have been proposed, from Levy's (2003) contention that negative attitudes stem from lifelong exposure to negative societal stereotypes, to Gilbert et al.'s (1993) claim that 'repeated exposure to assertions for which there is no evidence increases the likelihood that people will believe those assertions' (p.222). Negative attitudes persist despite government investment in high-profile educational campaigns (e.g. 'See Me' campaign - Scottish Executive 2003-2006; Changing Minds campaign - Crisp et al., 2005) designed to increase awareness of mental illness and combat stigma. However, while such campaigns may tackle negative societal stereotypes of mental illness in the general adult population, they typically fail to address issues of mental illness in later life, such as the impact of cohort beliefs (e.g. views of self-sufficiency or the 'stiff upper lip' mentality), or ageist misconceptions, which may impact upon decisions to seek treatment.

However, encouragingly, negative attitudes to mental illness, although resistant to change, typically do not exist in isolation. As indicated in Chapter 1, positive attitudes of older people towards mental illness have also been reported by several authors (e.g. Brockington et al., 1993; Davidson \& Connery, 2003), and are explained theoretically with reference to, for example, broader changes in societal attitudes (i.e. a general mellowing of attitudes over time or a 'positive cohort shift'; Currin et al., 1998), or a change in emphasis with age towards increased positivity (Carstensen et al., 1999). Thus, socio-emotional selectivity theory suggests a changing value base with age such that individual attention is directed towards 
emotionally salient and inherently positive aspects of life (Cartensen et al., 1999). The theory suggests that knowledge of limited time has direct effects on emotional experience such that older people are attuned to and remember emotionally positive information more readily than they do negative or neutral information. Perceived endings are proposed to bring out the best qualities in people such that kindness, for example, becomes a more prominent feature. Results from the current study may be usefully interpreted in this context since in addition to expressing fear of mental illness, older participants also expressed greater benevolence towards those who are ill (Brockington et al., 1993). A significantly larger proportion of older as opposed to younger participants also endorsed the view that people are generally sympathetic and caring towards those with mental health problems.

Yet, if it is indeed the case, as Carstensen et al. (1999) suggest, that older people become increasingly positive with age, one might reasonably expect differences in attitudes towards mental illness between successive generations of older adults, and between older and younger people. There was no support for either proposition in the current study (see also Pepin, 2006), although these results must be interpreted within the confines of cross-sectional research which precludes the assessment of change in attitudes over time. The perceived lack of enhanced positivity of attitudes with age is particularly noteworthy in light of Hayward and Bright's (1997) methodological contention that positive views of mental illness are more likely to occur in studies utilising a closed question methodology. 
As with hypotheses of increased positivity in later life, neither was there evidence of increased negativity of attitudes with age in the current older adult sample. Rather older peoples' attitudes to mental illness were multidimensional and consistent with those of younger people. This finding therefore qualifies previous research that assumes negativity on the part of older people but neglects to compare older peoples' views with those of a younger sample. However, it is also possible that older peoples' views may change when issues of mental health are considered within the broader context of ageing. In the focus group discussions, for example, participants were more explicitly positive in their attitudes towards the young in relation to mental illness, although it was not possible for survey respondents to qualify their responses in this manner.

Finally, Levy's (2003) work on stereotypes of ageing also has applicability to the current discussion of older peoples' attitudes to mental illness, since it suggests that a different pattern of results might emerge through utilisation of measures designed to access implicit as opposed to explicit attitudes (by using, for example, implicit association tasks). Thus, implicit negative attitudes may not be consciously accessible or may be masked by cultural norms of interaction requiring a more respectful approach (e.g. Boduroglu et al., 2006). This view accords with dual process models of the accessing and reporting of attitudes since it highlights the possibility of two qualitatively different mechanisms of information processing in relation to implicit attitudes (i.e. unconscious, automatic processing), and explicit attitudes (i.e. conscious, controlled processing). Studies should therefore be implemented to compare implicit and explicit attitudes of older people. 


\subsubsection{Hypothesis 2}

The question of whether experience of mental illness and contact with services exerts a positive or negative influence on attitudes is a complex one, since some studies report on the beneficial effects of increased familiarity and 'contact' between those with mental health difficulties and services (e.g. Huxley, 1993; Lasoski \& Thelen, 1987), while others espouse the danger of exposure to stigmatisation in already vulnerable individuals (e.g. Sartorius, 2003). In general, findings from the empirical literature suggest that older people, particularly those of a lower educational level and social class, are less favourable in their attitudes to mental illness, while experience increases favourable attitudes. Thus, Hayward and Bright (1997) have suggested that older people who have received relief from symptoms through treatment, tend to evaluate the experience positively. On the other hand, however, if stigma exists against the mentally ill, and a double stigma is evident against the elderly with mental health problems (Sartorius, 2003), the sharpest impact will presumably be felt in those currently attending mental health services. Crucial mediating factors in this regard appear to be the nature of the experience, the individual's perception of it, and whether or not services/treatment are perceived as helpful (Huxley, 1993; Leventhal et al., 1992).

In contrast to suggestions by Sartorius (2003) and others, and in accordance with the views of Huxley (1993), results from the current study indicated that attitudes to mental illness in later life were more negative in non service-users as opposed to service users. This is an interesting finding, since it highlights the possibility of a positive effect of service contact on attitudes, and the potential for positive 
experience to counteract the effects of internalised ageism (see Hypothesis 3). However, no significant inter-group differences in attitudes were found in relation to perception of stigma (i.e. as measured by BMHSS 'Stigma') or understandability associated with mental illness. While this may be indicative of more entrenched attitudes in these areas it may also simply reflect the poorer psychometric properties of these scales.

However, there are several caveats to the conclusion that experience results in positive attitudinal shift. First, as indicated above, it is not possible to ascertain causality in a cross-sectional correlational study, and so the precise nature of the relationship between experiences and attitudes cannot be determined. Second, it is unclear to what extent the result obtained is attributable to the noted age difference between the groups (i.e. non-clinical participants were, on average, older than clinical participants, and there were no significant inter-group differences when age was controlled). Currin et al. (1998) reported that earlier born cohorts tend to express more negative views in relation to mental illness since the attitudes of this cohort were formed at a time when societal views of mental health and mental illness were less accepting. Third, one cannot exclude the possibility that the results reflect the effects of dissonance on the part of service users. Cognitive dissonance theory suggests a change in individual reactions following exposure to information or experience which is discordant with the individual's previous frame of reference or knowledge (Ron, 2007). In this case, an older person who may have previously held negative attitudes to mental illness is exposed to a situation (i.e. personal diagnosis of mental illness/positive mental health service contact) which does not concord with 
his or her pre-existing knowledge or expectations. The psychological discomfort which ensues from the contradicting cognitions/experiences prompts the individual to change his or her previous attitudes to those that are more in line with current knowledge or experience. In the current context it is therefore plausible that service users were motivated to adopt more positive attitudes to mental illness as a result of the psychological discomfort incurred by dissonance experienced at first diagnosis.

Further analyses highlighted that individuals with prior experience of mental illness (whether direct or indirect) also endorsed more positive attitudes than those without such experience. Again, this result suggests a positive mediating effect of experience (and service contact) on attitudes to mental illness in later life. If, despite the caveats noted above, experience does indeed have a positive influence on attitudes to mental illness in later life it remains to be determined which particular aspects of the experience are most influential in relation to attitude formation. As this question was not addressed by the current study it is only possible to speculate about how or why experience might result in a positive attitudinal shift.

One such possibility is provided by the 'contact hypothesis' (Desforges et al., 1991). In essence, this theory draws upon models of prejudice and attitude change by proposing that attitude change occurs as a result of direct proximity of contact with the outgroup (i.e. in this case due to contact with older people with mental health difficulties rather than experience of services per se). Alternatively, one could argue that older people may have lower expectations of care and are less likely to perceive poor treatment due to what has been termed the 'gratitude factor' (Scottish 
Executive, 2002a). Results might also reflect the favourable impact of treatment for mental health problems in undermining negative expectations and beliefs, and potentially also in 'normalising' mental health difficulties. As indicated in focus group analyses, some participants believed that they would be treated unfavourably by health professionals by simple virtue of being an older person with a mental health problem. A positive experience of service contact might therefore go some way to displacing such beliefs.

Finally, an individual's health beliefs or attributions about their illness may affect how the individual perceives and deals with their illness (Halligan, 2007). It is important to recognise that individual illness beliefs may be influenced by prevailing negative societal views including those of healthcare professionals, all of which may be effectively challenged by positive contacts between services and patients. Clearly a better understanding of individual representations of illness and experiences of service contact are required to illuminate any potential pathway between experience and attitudes. While the non-clinical focus group narrative in the current study touched on some of these issues, addressing, for example, individual perceptions of the cause and consequence of mental illness, these issues represent fruitful areas for further research.

\subsubsection{Hypothesis 3}

As hypothesised, negative attitudes to mental illness were associated with negative attitudes to ageing across the entire sample. Specifically, there was an association 
between a loss-deficit view of ageing, or the perception of ageing as a time of deterioration and decline, and negative attitudes to mental illness such as understandability (see Section 3.2.3.3.2; Table 14). This finding accords with Law (2003), who found that the understandability phenomenon related to older peoples' attitudes towards their own ageing. Levy (2003) and Levy \& Banaji (2002), attribute negative attitudes to ageing to the effects of repeated exposure to negative societal stereotypes. It seems plausible then that negative implicit aging self-stereotypes could be activated by manifestations of other negative stereotypes that permeate society including those relating to mental illness. In this sense, the latter could act as a trigger of age-associated assumptions, for example, old age is a time of loss and decrepitude, and depression is a natural consequence of ageing (Laidlaw \& Baikie, 2007). Further support for this view derives from a study by Sarkisian et al., (2002) in which the views of 429 elders were sought regarding expectations of ageing. Results indicated that over half the sample felt it was an expected part of ageing to become depressed, one-quarter considered age-associated decline inevitable, and one-fifth of the sample did not expect to maintain health-related quality of life. Better physical and mental health was independently associated with higher expectations of ageing.

In line with Sarkisian et al's (2002) results, further exploration of the observed association between attitudes to mental illness and attitudes to ageing in the current study revealed a differential pattern of associations across clinical and non-clinical groups. Thus, the clinical group expressed significantly more negative attitudes to ageing (i.e. were more inclined to view ageing as a time of loss and decline) and 
significantly more positive attitudes to mental illness, while the converse was true for the non-clinical group. This pattern of results may be usefully interpreted with reference to models of successful ageing and self-regulation such as the SOC model (Baltes \& Smith, 2002), and the SRM model (Leventhal et al., 1992) described previously. The SOC model, for example, proposes that individuals strive to maintain homeostasis or equilibrium through self-regulatory processes by balancing gains and losses in later life using the strategies of selection (setting specific goals), optimisation (refining and enhancing means to attain the selected goals), and compensation (the creative use of alternative means to reach one's goals despite limited capacity). The SOC model can therefore be applied to ways of adapting to negative changes (such as a diagnosis of mental illness) as well as to describing pathways towards achievement of positive change (Ebner et al., 2006).

Jopp and Smith (2006) suggest that individuals low on resources will benefit from balancing losses through compensation and from enhancement of existing resources through optimisation compared with resource-rich individuals. In the context of the current research, it is possible that clinical participants in this study directed their resources towards maintenance of function rather than striving for personal growth and optimisation in the face of challenges to their mental health. In SOC terms this means that they may be less likely to experience themselves as ageing successfully and more likely to express a less positive perception of the ageing process than individuals who have yet to encounter significant challenges and losses. 
The relationship between personal experience and attitudes discussed previously can also be contextualised within the framework of the SOC. Experience of mental illness, or lack thereof, may not only account for some of the variance in attitudes to mental illness (as indicated by regression analyses) but also impact upon selfperceptions of ageing. Thus, lack of experience of mental illness may underlie the observed association, in non-clinical participants, between positive attitudes to physical aspects of ageing (i.e. perhaps as a result of better self-regulation in this area and consequently less contact with mental health services) and negative views of mental illness (see also Sarkisian et al., 2002). This view is further supported by demographic data indicating that non-clinical participants have higher reported subjective health status and, more importantly, by the noted relationship between positive attitudes to ageing and higher subjective well-being discussed in more detail in Hypothesis 4.

Of course, when considering characteristics of the study sample such as clinical status, it is also important to consider that the majority of the sample can be classified as young-old (i.e. under the age of 80) and thus, in terms of Baltes and Smith's (2002) model, at generally lower risk of experiencing the challenges of ageing. Thus, in the fourth age, 'it becomes conspicuous that sizeable aging losses do occur' (p7), necessitating a number of transitions which bring individuals face to face with experiences that may change their attitudes about several factors including mental health. As noted previously therefore, a changing pattern of associations between attitudes to ageing and attitudes to mental health in older people might be 
obtained over time and should therefore be investigated further in longitudinal research.

In summary, therefore, attitudes to mental illness and attitudes to ageing appear to be linked and the relationship between them potentially mediated by personal experience and capacity for self-regulation. The latter may of course be more difficult to achieve when faced with mental health difficulties, and potentially also as one grows older since the fourth age is a time of cumulative losses (Baltes \& Smith, 2002). Implementation of selection, optimisation and compensation strategies may therefore be crucial to ageing well when personal resources are constrained. This suggests that more attention should be paid to the development of such strategies in older individuals, particularly those facing the challenges associated with mental health problems. However, before such a proposal is taken forward, it is also important to consider alternative explanations for the observed results. Thus, one possibility is that positive attitudes to ageing endorsed by non-clinical participants simply reflect the views of a sample of relatively healthy, high functioning, individuals. In addition there is also the possibility that observed results (i.e. the association of negative attitudes to mental illness and negative attitudes to ageing) are a function of the propensity towards negative affectivity in some individuals (Teachman, 2006), although this does not explain the observed pattern of inter-group results obtained in this study. 


\subsubsection{Hypothesis 4}

Across the study sample positive attitudes to ageing, specifically attitudes relating to personal growth and health, significantly predicted engagement in health-related behaviours, while negative attitudes to ageing and (to a lesser extent) to mental illness significantly predicted lower subjective well-being (see Section 3.2.3.4.2). Participants in the clinical group expressed more negative attitudes to ageing, higher internalised ageism, and reported poorer psychological health, while non-clinical participants expressed more positive attitudes to ageing, more engagement in healthrelated behaviours, and reported better psychological health. Interestingly, in terms of health behaviours, and in line with the noted potential importance of selfregulation, clinical participants also endorsed far fewer cognitive and affective regulation strategies (for example, were less likely to think positively, or to avoid emotional distress) than non-clinical participants (see Section 3.2.3.4.1; Table 16).

Although the above results should be interpreted cautiously due to lack of sufficient power and potential confounding of measures, these findings are nevertheless important since they suggest that perception of ageing is particularly influential in terms of health outcomes in older people. On the basis of these results, one could argue that low age expectations may act as a potential barrier to engagement in health-related behaviours (Sarkisian et al., 2002). Identifying the causes of underuse of mental health services by older people is of critical importance so that interventions may be designed to ensure that all older people are given the opportunity to receive care likely to improve their health and well-being. Older people may attribute mental health problems to ageing as a useful coping mechanism 
in the face of unmodifiable illness, however older people with low expectations of ageing are likely to have lower rates of use of appropriate mental health services and may therefore forego effective treatments for modifiable conditions such as depression and anxiety (Sarkisian et al., 2002).

In the literature low age expectations are reported as highly predictive of morbidity and mortality beyond other predictors such as age (Levy 1996; Levy \& Banaji, 2002), leading Jopp and Smith (2006) to appeal for research identifying factors that help to maintain a positive attitude to ageing. In their landmark paper, Rowe and Kahn (1987) defined successful ageing as absence of disease, disability, and risk factors; maintenance of cognitive and physical functioning; and active engagement with life. It is clear from the current research that not all older individuals can achieve and maintain this ideal, and more work is clearly needed to develop strategies to cultivate positive attitudes to ageing in the face of potential challenges and losses.

\subsection{CLINICAL AND SERVICE IMPLICATIONS}

\subsubsection{Clinical considerations}

From a clinical perspective it is important to understand the specific nature of the relationship between ageist attitudes and attitudes towards mental illness, and the ways in which attitudes impact upon the manifestation and presentation of different psychological problems. For example, if older people who have negative attitudes to 
ageing also hold lower expectations of health-related quality of life, to what extent, if any, does this impact upon their propensity to seek care for mental health problems? Little is known about the origins of negative beliefs of ageing and mental illness which may be partly attributable to cohort effects or to lack of awareness of the potential value of efficacious psychotherapeutic treatments. Alternatively, negative attitudes may be an important coping mechanism by which many older people are able to maintain a degree of life satisfaction in the face of adversity. The latter possibility is of particular relevance to the development of an effective therapeutic intervention since deconstructing such beliefs without due care may leave the older client vulnerable to further distress.

It would therefore be prudent for clinicians to ascertain client beliefs about illness and ageing prior to embarking on treatment. Intervention that does not take account of this information is likely to have little impact and may lead to poor adherence to psychological interventions (Lobban et al., 2003). Laidlaw and Baikie (2007) argue that therapists must also expand their knowledge and understanding of gerontology in order to better serve their older clients. As Laidlaw et al. (2004) state:

"The age stereotype of ageing equates this phase of life with decrepitude, and this needs to be addressed in order for a patient to challenge erroneous thinking that may prevent him from fully making changes in his life." (p.397)

\subsubsection{Service and Policy Development}

The current research also has several important public health education implications. These include the continued need to destigmatise and demystify mental illness in 
later life by addressing specific symptoms, course, presentation, and management of common problems such as depression. If experience of illness and service contact contributes to improved attitudes, it becomes ever more important to increase awareness of, access to and provision of mental health services for older people. This fits neatly with a number of current policy drivers (e.g. Scottish Executive, 2006; 2007), however, few, if any, of the existing health policies and educational campaigns address mental illness within the broader context of ageing. Given that the Scottish Executive (2004) itself reports that older people per se are less receptive to educational campaigns, this seems to warrant more strategically targeted initiatives aimed at this growing sector of the population.

To this end, perhaps greater efforts could be made to incorporate facets of models of successful ageing and self-regulation into public educational initiatives (e.g. Baltes \& Smith, 2002; Leventhal et al., 1997). Specifically, information should be provided on strategies which enable the older person to optimise their ageing experience in the face of particular challenges and losses such as those posed by mental health difficulties. Since changes in attitudes do not always typically equate to behavioural change, educational programmes must also address individual health and illness beliefs such as self-efficacy, causality and control which may provide the motivational basis for individual behaviour change (Jopp \& Smith, 2006). As another important factor potentially impacting on outcome, beliefs about older people with mental health problems held by professionals should also be researched and addressed. 


\subsection{METHODOLOGICAL CRITIQUE}

\subsubsection{Mixed Methodology Design}

As noted throughout, there are a number of limitations to the current study. In terms of study design, it was originally intended to undertake focus groups primarily to pilot the study questionnaire, and to briefly explore the subject area prior to survey. However, it quickly became clear that even a cursory discussion of the study topic with small groups of participants yielded a great deal of information that was worthy of further analysis. The qualitative element of the study therefore expanded beyond what was originally anticipated with a consequent number of advantages and disadvantages. At one level the mixed methodology design provided the opportunity for a richer understanding of a complex and under-researched area in relation to older people, raising several potential areas for further study. Such an approach is entirely in-keeping with recommendations in the literature which advocate the advantages of combined approaches in the study of psychosocial phenomena (e.g. Wilkinson, 2003). At another level, however, the design of the study was such that the qualitative element may not have been sufficiently integrated into the overall design, leading to a disjuncture between the qualitative and quantitative arms of the study. For example, it was not possible to pick up on and further expand upon some of the issues raised during focus group discussions in subsequent stages of the study.

Wilkinson (2003) further advocates that focus groups are unlikely to be useful if statistical and generalisable findings are required, although the triangulation of 
methods used in this study goes some way towards mitigation of this critique. Moreover, Haug (1987, p.44) notes that 'if a given experience is possible, it is also subject to universalisation'. In the current study only two focus groups were conducted. Participants were self-selecting, and no attempt was made to stratify groups according to age, sex, race, or length of contact with mental health services. Thus, participants may not have been representative of the populations from which they were drawn. These limitations would, however, only prove a substantial threat to the validity of study findings if the object of the focus group was to do anything other than provide a platform for the basis of the quantitative study.

There was also a range of problems with the chosen method of analysis such as potential loss of detail, failure to capture focus group processes (e.g. nuances of participant interaction), and the potential for bias in results due to what has been termed the 'double hermeneutic' of interpretive activity (Smith et al., 1999). That is, results are likely to reflect the assimilation of respondent interpretation of questions and researcher interpretation of answers. However, one could counter argue that the fundamental nature and strength of qualitative research is that it is both an interpretive and subjective exercise where the researcher is intimately involved in, and not remote from, the process (Lacey \& Luff, 2001). 'Reflexivity', or the researcher's ability to reflect on what they bring to the research, has therefore been regarded as essential for good practice (Willig, 2001). 


\subsubsection{Main Study}

\subsubsection{Sampling Issues}

With reference to the main study, a number of additional limitations apply. Overall response rate was low despite attempts to improve sample size, and it was not possible to ascertain reasons for non-participation. It is therefore likely that there was a degree of bias or selectivity in the sample of individuals who consented to participate, rather than the study sample being truly representative of the larger population. Individuals who responded to the questionnaires might have differed from non-responders with respect to such attributes as helpfulness, willingness to report on personal experiences, or through a sense of duty to advocate on behalf of other, perhaps less able, older people. Conversely, it may be that some individuals who refused to take part in the study were influenced in this decision by personal sensitivities to the subject area (Crisp et al., 2000). However, given the voluntary nature of participation in research, it would be extremely difficult to attempt to control for such factors in future studies.

Due to the method of recruitment, and despite participants from both groups reporting a broad range of health conditions, it is likely that the non-clinical sample (and to some extent, also the clinical sample) comprised relatively high functioning and articulate individuals who were able to reflect upon and discuss their attitudes in relation to mental illness. In particular, the clinical sample may have been biased towards those who were relatively well engaged with services and were active service users, thus representing a particular subgroup within the broader older adult 
population with mental health difficulties who place greater importance on seeking health-care. Potential participants may also have been differentially excluded due to differing clinician interpretations of the level of cognitive impairment required for exclusion from study participation. Conversely, it is also possible that, despite exclusion criteria, some participants were included in the study sample who suffered from a degree of cognitive impairment. Nevertheless, while groups may be neither totally representative of local mental health service users nor of the lay community this did not appear to mitigate against the endorsement of negative attitudes as reported in the study.

\subsubsection{Cross-sectional Design}

As the current study was cross-sectional, involving assessment of all variables of interest at the same time point, it was not possible to provide evidence about the causal mechanisms connecting study variables. For example, whether having negative attitudes actually causes older people to seek less health care for mental health difficulties for modifiable conditions remains unclear. The study design also precludes the opportunity to ascertain if between-group attitudinal differences were the result of cohort effects (i.e. the non-clinical group were older on average) or developmental patterns likely to change over time. The relatively small sample size and imbalanced group numbers also prevents definitive conclusions being reached in relation to study hypotheses, highlighting the importance of replication of study findings under more statistically rigorous conditions. In particular, in order to increase numbers of clinical participants and potentially provide a more 
representative sample of mental health service users, attention should be paid to eliciting the support of psychiatry colleagues in sample acquisition as this was not successfully achieved in the current study. Typical reasons given were demanding workloads, not enough time, forgetting to distribute study invitation packs, and the study not being a clinical priority. It is hoped that feedback of study results to mental health service colleagues will go some way towards contradicting this latter point.

\subsubsection{Measures}

A general limitation relating to the use of self-report questionnaires is the possibility of inflation of the relationships between the variables due to common method variance (i.e. variance that is attributable to the method of measurement rather than to the constructs being measured; Podsakoff et al., 2003). Results from the regression analysis also indicated varying degrees of inter-correlation between attitudinal measures of mental illness in later life and ageing, suggesting potential confounding of measures, and, at the very least, warranting caution in interpretation of study results. However, the complexity of attitudinal research is such that it would be difficult to control for the effects of confounding using self-report measures.

Regarding psychometric properties of measures, several measures lacked sufficient information about reliability and validity. The BMHSS (Pepin, 2006) was included in the study with the permission of the author on the understanding that sub-scales could be used independently to investigate the role of particular clusters of attitudes to mental illness. However, no data have yet been published in peer-reviewed 
journals regarding the psychometric properties of the BMHSS. Missing responses for several BMHSS sub-scale items were relatively high, and indicative of poorly worded and ambiguous items (a view supported by comments obtained during questionnaire piloting).

The Understandability measure (Law, 2003) is not a standardised measure and demonstrated poor internal consistency within the current sample. Similarly, the Rame questionnaire (Parnell et al., 2001), although achieving high internal reliability in the current study, has not been standardised on a large older adult sample. These factors necessarily limit interpretation and generalisability of results, indicating that both measures require more rigorous psychometric testing if they are to be included in future studies.

The AAQ (Laidlaw et al., 2007) has much stronger psychometric properties than most other measures in the study having been developed through a combination of both modern and classical analytical methods. However, although a three-factor model of successful ageing can be statistically derived from the measure, the theoretical basis for such a model is lacking.

The GHQ-12 (Goldberg, 1992) is a well-validated measure of psychiatric morbidity with a strong evidence base in clinical research. However, the majority of research has been conducted with younger adults (e.g. Johnston et al., 1995), raising questions of the lack of standardisation and applicability of the measure to an older adult population. Although criticised for being a measure of negative well-being (Huppert \& Whittington, 2003), the Likert method of scoring the GHQ-12 allows for a broader assessment of subjective well-being and was used in this study since it also 
reportedly gives a less skewed distribution of scores. However, since cut-off thresholds have not been validated for Likert scoring, and scores are likely to be inflated due to the presence of somatic symptoms in older people suffering from depression or anxiety, it is difficult to know whether participant scores could be considered clinically relevant.

Finally, the RHBC (Prohaska et al., 1985) was originally published as a 21-item selfreport measure of health-related behaviours in which items were scored individually. As such, the authors provided no data regarding the psychometric properties of the scale. More recently the scale has been utilised to produce both a composite score, and sub-scales relating to 'Health-Action' and 'Cognitive-Affective' domains (Kincey et al., 2003). Although these sub-scales appear to make conceptual sense, there are no published details of how they were derived and if indeed they are supported statistically. This suggests the need for further clarification of the measure in the empirical literature. The relatively low internal consistency of the 'Health Action' scale in the current study sample supports this suggestion.

\subsection{RECOMMENDATIONS FOR FUTURE RESEARCH}

The present study overcomes some of the problems of previous studies which rely exclusively on younger adults' evaluations and self-reported attitudes to mental illness in older people, and as such offers a useful platform fur future research in this area. Due to the methodological and statistical limitations of the study noted above, a replication and/or extension of the study is required in order to draw firmer conclusions regarding the potential interplay between attitudes to mental illness and 
ageing, and behaviour. Indeed, the complexity of the issues being considered is such that relationships may only be properly teased out through prospective longitudinal research. In addition, as attitudes are complex phenomena, they are unlikely to be adequately described by single-strand methodologies. Recommendations for future research therefore include the implementation of multimethod studies combining qualitative (for example, using grounded theory or interpretive phenomenological analysis; Smith et al., 1999) and quantitative approaches in a more cohesive fashion than was employed here.

Since attitudes about mental illness and ageing may affect both treatment-seeking behaviour and treatment provision, studies are required to alert individuals, health professionals and health policy makers to potential barriers which may prevent older people from accessing mental health services. Further work should also seek to identify strategies to facilitate a more successful ageing experience for older individuals with mental health difficulties, and to address the link between attitudes to mental illness and health-related behaviours. For example, do those who attribute depression to a normal part of the ageing process actually fail to seek appropriate care? Similarly, in relation to the viability of different models of health beliefs to explain help-seeking behaviour in older people with mental illness, little is known about the specific beliefs and expectations held by this population, or of the beliefs and expectations held by family members or carers which may impact upon the individual's propensity to seek help (Lobban et al., 2003).

The present study also raises issues regarding measurement of explicit as opposed to implicit attitudes. Implicit attitudes may differ from explicit attitudes, although 
measuring implicit attitudes is difficult since they may not be consciously accessible to the individual (Levy, 2003). As such, typical self-report (i.e. explicit) measures of attitudes and stereotypes are unsuitable for this purpose and may be influenced by social desirability factors or the need for participants to convey that they are unprejudiced or unbiased. Further research of both implicit and explicit attitudes is therefore required using appropriate methodologies (see Hummert et al., 2002).

Finally, cross-cultural studies of attitudes to ageing and mental illness are required in order to identify the broader context in which attitudes develop and hence to identify any potentially mitigating factors against the cultivation of inherently negative views of older people (Boduroglu et al., 2006). The examination of cultural differences in perceptions of ageing has thus far been relatively rare in the literature but is nevertheless important to highlight the fact that negative views of ageing are not universal, and are therefore modifiable.

\subsection{SUMMARY AND CONCLUSIONS}

That negative stereotypes exist both in relation to mental illness and to ageing is well documented (e.g. Crisp et al., 2005., Hayward \& Bright, 1997; Levy, 2003). However, little empirical research to date has explored the possibility that attitudes to mental illness in later life are actually a reflection of broader (i.e. underlying) attitudes towards ageing and/or are a reflection of personal experience. The attitudinal research that has been undertaken has rarely compared the views of older and younger adults (e.g. Lundervold \& Young, 1992), and no studies could be 
identified in the literature comparing the views of older people with and without mental health difficulties. In line with indications in the literature that negative attitudes may contribute a significant barrier to help seeking for mental health problems in later life (e.g. Pepin, 2006; Sarkisian et al., 2003), older peoples' attitudes to mental illness were explored in relation to attitudes to ageing, experience, and health-related outcomes.

Notwithstanding the limitations of the study, results indicated that older and younger adults endorsed a range of positive and negative views of mental illness, and that the oldest-old were not inherently more negative in their attitudes than the young-old. However, when attitudes to mental illness were considered within the broader context of ageing and personal experience, a more complex pattern of results emerged. Across the entire sample, as expected, negative attitudes to ageing were associated with negative attitudes to mental illness. However, differential effects were found in relation to study groups. Specifically, participants who were, to all intents and purposes, ageing well (i.e. the non-clinical group), and who therefore endorsed more positive attitudes to ageing, also reported more negative attitudes to mental illness. Conversely, participants currently attending services for mental health difficulties (and those with prior experience of mental illness) reported more positive attitudes to mental illness, and more negative attitudes to ageing. It was proposed that attitudes to mental illness and ageing are linked and mediated by personal experience and capacity for psychological self-regulation. In terms of further research it would therefore be of interest to assess potential differences in coping strategies and health behaviours of individuals who endorse generally positive or negative attitudes to ageing when faced with age-associated challenges. 
Several important implications from these study findings can be inferred. Not least, negative attitudes may pose significant barriers to help-seeking, and/or engagement in proactive health-related behaviours, and result in poorer subjective well-being. Further work is therefore required to explore patterns of interaction between these variables. Such work might address, for example, whether attitudes to ageing are triggered by individual experiences of mental illness, and whether subjective wellbeing is an outcome and/or a predictor of attitudes. A number of immediate concerns relating to the present findings are also raised. For older people, these include the potential failure to recognise symptoms of mental illness, and the disinclination to seek help for potentially modifiable and treatable conditions. This lends support to calls for improved and comprehensive screening of older people for mental health difficulties in primary care settings (Sarkisian et al., 2003). For clinicians, results highlight the importance of understanding older clients in terms of cohort factors (Seedsman, 2005), and within a broader gerontological context, since such factors have the potential to influence the process and outcome of therapy (for example, by hindering engagement in therapy and lowering expectations of treatment success). Finally, for health policy makers, study findings point to the necessity of improving awareness of and access to mental health services for older people, and of developing and implementing targeted health education interventions which demystify mental illness in later life, address ageist misconceptions, and prioritise the needs of older people in the mental health agenda. The demographic imperatives of ageing societies are such that a qualitative shift in emphasis is now required away from a focus on lengthening life to increasing years of health and well-being. 


\section{5 - REFERENCES}

Allsup, S.J. \& Gosney, M.A. (2006). Anxiety and depression in an older research population and their impact on clinical outcomes in a randomised controlled trial. Postgraduate Medical Journal, 78, 674-677.

American Psychiatric Association. (2000). Diagnostic and Statistical Manual of Mental Disorders ( $4^{\text {th }}$ edn, Text Revision). Washington: Author.

Baltes, P.B. \& Smith, J. (2002). New frontiers in the future of aging: from successful aging of the young old to the dilemmas of the fourth age. Gerontology, 49 (2), 123-135.

Beekman, A.T.F., Deeg, D.J.H., van Tilburg, T., Smit, J.H., Hooijer, C. \& van Tilburg, W. (1995). Major and minor depression in later life: a study of prevalence and associated factors. Journal of Affective Disorders, 36 (1-2), $65-75$.

Beekman, A.T.F., Copeland, J.R.M. \& Prince, M.J. (1999). Review of community prevalence of depression in later life. The British Journal of Psychiatry, 174 (4), 307-311.

Beekman, A.T.F., de Beurs, E., van Balkom, A.J.L.M., Deeg, D.J.H., van Dyck, R. $\&$ van Tilburg, W. (2000). Anxiety and depression in later life: cooccurrence and communality of risk factors. American Journal of Psychiatry, 157, 89-95.

Birrer, R.B. \& Vemuri, S.P. (2004). Depression in later life: a diagnostic and therapeutic challenge. American Family Physician, 69 (10), 2375-2382.

Blanchard, M (1992). The elderly. International Review of Psychiatry, 4 (3-4), 251256. 
Blank, K., Cohen, C.I., Cohen, G.D., Gaitz, C., Gottlieb, G.L., Katz, R.R. et al. (2001). Failure to adequately detect suicidal intent in elderly patients in the primary care setting. Clinical Geriatrics, 9 (1), 26-36.

Blazer, D.G. (2002). The prevalence of depressive symptoms. Journals of Gerontology Series Medical Sciences, 57A (3), M150-M151.

Blazer, D.G. (2003). Depression in late life: review and commentary. Journal of Gerontology: Medical Sciences, 58A (3), 249-265.

Blazer, D.G. (2006). Successful ageing. American Journal of Geriatric Psychiatry, $14(1), 2-5$.

Blazer, D.G. \& Williams, C. (1980). The epidemiology of dysphoria and depression in an elderly population. American Journal of Psychiatry, 137 (4), 439-444.

Blazer, D.G., Bachar, J.R. \& Hughes, D.C. (1987). Major depression with melancholia: a comparison of middle-aged and elderly adults. Journal of the American Geriatrics Society, 35, 927-932.

Blazer, D.G., Burchett, B., Service, C. \& George, L.K. (1991). The association of age and depression among the elderly: an epidemiologic exploration. Journal of Gerontology, 46 (6): M210-M215.

Boduroglu, A., Yoon, C., Luo, T. \& Park, D.C. (2006). Age-related stereotypes: a comparison of American and Chinese cultures. Gerontology, 52 (5), 324333.

Bostock, Y. \& Miller, C. (2003). Mental health and well-being in later life: older people's perceptions. Edinburgh: Health Scotland. 
Bowling, A. (1999). Ageism in cardiology. British Medical Journal, 319 (7221), 1353-1355.

Brockington. I.F., Hall, P., Levings, J. \& Murphy, C. (1993). The community's tolerance of the mentally ill. British Journal of Psychiatry, 162, 93-99.

Butler, R.N. (1969). Ageism: Another form of bigotry. Gerontologist, 9, 243-246.

Butler, R.N. (1993). Dispelling ageism: the cross-cutting intervention. In D. Shenk \& W.A. Achenbaum (Eds.) Changing perceptions of aging and the aged (pp. 75-82). Generations: Journal of the American Society on Aging, XV11 (2). NY: Springer.

Byrne, P. (1997). Psychiatric stigma: past, passing, and to come. Journal of the Royal Society of Medicine, 90, 618-620.

Byrne, P. (2001). Psychiatric stigma. British Journal of Psychiatry, 178 (3), 281284.

Carstensen, L.L. \& Mikels, J.A. (2005). At the intersection of emotion and cognition: Aging and the positivity effect. Current Directions in Psychological Science, 14 (3), 117-121.

Carstensen, L.L., Isaacowitz, D.M. \& Charles, S.T. (1999). Taking time seriously. A theory of socioemotional selectivity. American Psychologist, 54 (3), 165181.

Census (2001). Population Data. Retrieved December 2006 from: (http://www.statistics.gov.uk/census2001/census2001.asp)

Chew-Graham, C., Baldwin, R. \& Burns, A. (2004). Treating depression in later life. British Medical Journal, 329 (7459), 181-182. 
Clark-Carter, D. (2004). Quantitative psychological research: a student's handbook. Hove: Psychology Press.

Cohen, B. (1999). Measuring the willingness to seek help. Journal of Social Service Research, 26 (1), 67-82.

Cohen, J. (1988). Statistical power analysis for the behavioural sciences $\left(2^{\text {nd }}\right.$ edn). Hillsdale, New Jersey: Lawrence Erlbaum Associates, Inc.

Cohen, J. (1992). A power primer. Psychological Bulletin, 112 (1), 155-159.

Cole, M. G., \& Bellavance, F. (1997). The prognosis of depression in old age. American Journal of Geriatric Psychiatry, 5, 4-14.

Connery, H. \& Davidson, K.M. (2006). A survey of attitudes to depression in the general public: a comparison of age and gender differences. Journal of Mental Health, 15 (2), 179-189.

Copeland, J.R, Dewey, M., Wood, N., Searle, R., Davidson, I. \& McWilliam, C. (1987). Range of mental illness among the elderly in the community: prevalence in the Liverpool area using the GMS-AGECAT package. British Journal of Psychiatry, 150, 815-823.

Copeland, J.R.M., Beekman, A.T.F., Dewey, M.E., Hooijer, C., Jordan, A., Lawler, B.A. et al. (1999). Depression in Europe: geographic distribution among older people. British Journal of Psychiatry, 174 (4), 312-321.

Crawford, M., Prince, M., Menezs, P., \& Mann, A. (1998). The recognition and treatment of depression in older people in primary care. International Journal of Geriatric Psychiatry, 13 (3), 172-176. 
Crisp, A.H. (1999). The stigmatization of sufferers with mental disorders. British Journal of General Practice, 49 (438), 3-4.

Crisp, A.H., Gelder, M.G., Rix, S., Meltzer, H.I. \& Rowlands, O.J. (2000). Stigmatisation of people with mental illnesses. British Journal of Psychiatry, 177, 4-7

Crisp, A.H., Gelder, M.G., Goddard, E. \& Meltzer, H. (2005). Stigmatization of people with mental illnesses: a follow-up study within the Changing Minds campaign of the Royal College of Psychiatrists. World Psychiatry, 4 (2), 104-113.

Currin, J.B., Hayslip, B. Jr., Schneider, L.J. \& Kooken, R.A. (1998). Cohort differences in attitudes toward mental health services among older persons. Psychotherapy, 35 (4), 506-518.

Davidson, K.M. \& Connery, H. (2003). A Scottish survey of attitudes to depression in older and younger adults. Journal of Mental Health, 12 (5), 505-512.

De Beurs, E., Beekman, A.T.F., van Balkom, A.J.L.M., Deeg, D.J.H., van Dyck, R. \& van Tilburg, W. (1999). Consequences of anxiety in older persons: its effect on disability, well-being and use of health services. Psychological Medicine, 29 (3), 583-593.

Degnen, C. (2007). Minding the gap: The construction of old age and oldness amongst peers. Journal of Aging Studies, 21 (1), 69-80.

Department of Health. (2002). Health Survey for England 2000: Psychosocial wellbeing among older people. London: The Stationery Office.

Department of Health. (2003). Attitudes to Mental Illness Report. London: Office of National Statistics. 
Depp, C.A. \& Jeste, D.V. (2006). Definitions and predictors of successful aging: a comprehensive review of larger quantitative studies. The American Journal of Geriatric Psychiatry, 14 (1), 6-20.

Desforges, D.M., Lord, C.G., Ramsey, S.L., Mason, J.A., Van Leeuwen, M.S., West, S.C. et al. (1991). Effects of structured cooperative contact on changing negative attitudes toward stigmatised social groups. Journal of Personality and Social Psychology, 60 (4), 531-544.

Ebner, N.C., Freund, A.M. \& Baltes, P.B. (2006). Developmental changes in personal goal orientation from young to late adulthood: from striving for gains to maintenance and prevention of losses. Psychology and Aging, 21 (4), 664-678.

Field, A. (2005). Discovering statistics using SPSS (2 $\left.2^{\text {nd }} e d n\right)$. London: Sage Publications Ltd.

Flint, A.J. (1997). Epidemiology and comorbidity of anxiety disorders in later life: implications for treatment. Clinical Neuroscience, 4 (1), 31-36.

Fogel, B.S. \& Fretwell, M. (1985). Reclassification of depression in the medically ill elderly. Journal of the American Geriatrics Society, 33, 446-448.

Gallo, J.J., Rabins, P.V., Lyketsos, C.G., Tien, A.Y. \& Anthony, J.C. (1997).

Depression without sadness: functional outcomes of nondysphoric depression in later life. Journal of the American Geriatrics Society, 45 (5), 570-578.

Gilbert, D .T., Tafarodi, R.W. \& Malone, P.S. (1993). You can't not believe everything you read. Journal of Personality and Social Psychology, 65 (2), 221-233. 
Gilbert, P. (2000). Stigmatization as a survival strategy: "skeletons in the cupboard" and the role of shame. In A.H. Crisp. (Ed.) Every Family in the Land: understanding prejudice and discrimination against people with mental illness (Chapter 3). London: Royal Society of Medicine Press Ltd. Retrieved February 2007 from: (http://www.stigma.org).

Gilhooly, M. (2001). Ageism in the NHS? Report to the Chief Medical Officer's Expert Group on Health Care of the Elderly. Supporting Documentation to the Report “Adding Life to Years". Scottish Executive (2002a). Retrieved February 2007 from: (http://www.scotland.gov.uk/health/cmo/policyandresources.asp).

Goffman, E. (1968). Stigma: Notes on the management of spoiled identity. London: Penguin.

Goldberg, D. (1978). General Health Questionnaire (GHQ-60). Windsor: NFERNELSON.

Goldberg, D. (1992). General Health Questionnaire (GHQ-12). Windsor: NFERNELSON.

Green, S.B. (1991). How many subjects does it take to do a regression analysis? Multivariate Behavioral Research, 26 (3), 499-510.

Halligan, P. (2007). Belief and illness. The Psychologist, 20 (6), 358-361.

Harwood, J., Giles, H., McCann, R.M., Cai, D., Somera, L.P., NG, S.K. et al. (2001). Older adults' trait ratings of three age-groups around the Pacific rim. Journal of Cross-Cultural Gerontology, 16 (2), 157-171.

Haug, F. (Ed.). (1987). Female Sexualisation. London: Verso. 
Hawthorne, G., Davidson, N., Quinn, K., McCrate, F., Winkler, I., Lucas, R. et al. (2006). Issues in conducting cross-cultural research: implementation of an agreed international protocol designed by the WHOQOL Group for the conduct of focus groups eliciting the quality of life of older adults. Quality of Life Research, 15 (7), 1257-1270.

Hayward, P. \& Bright, J.A. (1997). Stigma and mental illness: A review and critique. Journal of Mental Health, 6 (4), 345-354.

Hinrichson, G. A. (2000). Interpersonal psychotherapy for treating late life depression. In L. Vandecreek and T. L. Jackson (Eds.) Innovations in clinical practice: A sourcebook (pp. 21-31), vol. 18. Sarasota: Professional Resource Press, 2000.

Hirai, N. \& Clum, G.A. (2000). Development, reliability, and validity of the Beliefs Toward Mental Illness Scale. Journal of Psychopathology and Behavioural Assessment, 22 (3), 221-236.

Hook, A. \& Andrews, B. (2005). The relationship of non-disclosure in therapy to shame and depression. British Journal of Clinical Psychology, 44 (3), 425-438.

Hummert, M.L., Garstka, T.A., Greenwald, A.G., Mellot, D.S. \& O'Brien, L.T. (2002). Using the implicit association test to measure age differences in implicit social cognitions. Psychology and Aging, 17 (3), 482-495.

Huppert, F.A. \& Whittington, J.E. (2003). Evidence for the independence of positive and negative well-being: Implications for quality of life assessment. British Journal of Health Psychology, 8, 107-122.

Huxley, P. (1993). Location and stigma: A survey of community attitudes to mental illness - Part 1. Enlightenment and stigma. Journal of Mental Health, 2, 7380 . 
James, A. (1998). Stigma of mental illness: Foreward. Lancet, 352, 1048.

Janssen, J., Beekman, A.T.F., Comjis, H.C., Deeg, D.J. \& Heeren, T.J. (2006). Latelife depression: the differences between early- and late-onset illness in a community based sample. International Journal of Geriatric Psychiatry, 21 (1), 86-93.

Jeste, D.V., Alexopoulos, G.S., Bartels, S.J., Cummings, J.L., Gallo, J.J., Gottlieb, G. et al. (1999). Consensus statement on the upcoming crisis in geriatric mental health. Archives of General Psychiatry, 56 (9), 848-852.

Johnston, M., Wright, S. \& Weinman, J. (1995). Measures in health psychology: a user's portfolio. Stress, emotion and life events. Windsor: NFER-NELSON.

Jopp, D. \& Smith, J. (2006). Resources and life-management strategies as determinants of successful aging: on the protective effect of selection, optimization, and compensation. Psychology and Aging, 21 (2), 253-265.

Jorm, A.F., Korten, A.E., Jacomb, P.A., Christensen, H., Rodgers, B. \& Pollitt, P. (1997). "Mental health literacy": a survey of the public's ability to recognise mental disorders and their beliefs about the effectiveness of treatment. Medical Journal of Australia, 166, 182-186.

Karel, M.J. (1997). Aging and depression: vulnerability and stress across adulthood. Clinical Psychology Review, 17 (8), 847-879.

Katon, W.J., Lin, E., Russo, J. \& Unützer, J. (2003). Increased medical costs of a population-based sample of depressed elderly patients. Archives of General Psychiatry, 60 (9), 897-903. 
Kincey, J., Mandal, D. \& Sinha, S. (2003). Relationships between anxiety, coping and diagnosis at initial attendance at a genito-urinary medicine clinic. Psychology, Health and Medicine, 8 (2), 205-211.

Kinsella, K. \& Velkoff, V.A. (2001). An Aging World. US Census Bureau, Series P95/01-1. Washington, DC: US Government Printing Office.

Kite, M.E. \& Johnson, B.T. (1988). Attitudes toward older and younger adults: a meta-analysis. Psychology and Aging, 3 (3), 233-244.

Kite, M.E., Deaux, K. \& Miele, M. (1991). Stereotypes of old and young: does age outweigh gender? Psychology and Aging, 6 (1), 19-27.

Kite, M.E., Stockdale, G.D., Whitley, B.E. Jr. \& Johnson, B.T. (2005). Attitudes toward younger and older adults: an updated meta-analytic review. Journal of Social Issues, 61 (2), 241-266.

Kitzinger, J. (1995). Introducing focus groups. British Medical Journal, 311 (7000), 299-302.

Kline, P. (1999). The handbook of psychological testing ( $2^{\text {nd }}$ edn). London: Routledge.

Kneebone I.I. \& Dunmore, E. (2000). Psychological management of post-stroke depression. British Journal of Clinical Psychology, 39 (1), 53-65.

Knight, B.G. (1999). The scientific basis for psychotherapeutic interventions with older adults: an overview. Psychotherapy in Practice, 55 (8), 927-934.

Knight, B.G. (2004). Psychotherapy with older adults $\left(2^{\text {nd }}\right.$ edn). London: Sage Publications, Inc. 
Kramer, M. (1983). The increasing prevalence of mental disorders: a pandemic threat. Psychiatric Quarterly, 55 (2\&3), 115-143.

Kreuger, R.A. (1994). Focus groups: a practical guide for applied research $\left(2^{\text {nd }}\right.$ edn). Newbury Park: Sage Publications Ltd.

Lacey, A. \& Luff, D. (2001). Qualitative data analysis. Sheffield: Trent Focus.

Laidlaw, K. \& Baikie, E. (2007). Psychotherapy and demographic change. Nordic Psychology, 59, 45-58.

Laidlaw, K., Thompson, L.W., Dick-Siskin, L. \& Gallagher-Thompson, D. (2003). Cognitive-behaviour therapy with older people. Chichester: John Wiley \& Sons Ltd.

Laidlaw, K., Thompson, L.W. \& Gallagher-Thompson, D. (2004). Comprehensive conceptualisation of cognitive behaviour therapy for late life depression. Behavioural and Cognitive Psychotherapy, 32 (4), 389-399.

Laidlaw, K., Power, M.J., Schmidt, S. \& the WHOQOL-OLD Group. (2007). The attitudes to ageing questionnaire (AAQ): development and psychometric properties. International Journal of Geriatric Psychiatry, 22, 367-379.

Lasoski, M.C. \& Thelen, M.H. (1987). Attitudes of older and middle-aged persons toward mental health intervention. The Gerontologist, 27 (3), 288-292.

Law, J. (2003). The 'understandability' phenomenon: Do older adults believe depression is a normal part of old age? Unpublished clinical doctoral thesis. University of Edinburgh: Edinburgh. 
Lebowitz, B.D. \& Niederehe, G. (1992). Concepts and Issues in Mental Health and Aging. In J.E. Birren., R. Bruce Sloane \& G.D. Cohen (Eds.) Handbook of mental health and aging (2nd edn., pp. 3-23). London: Academic Press, Inc.

Leventhal, H., Diefenbach, M. \& Leventhal, E.A. (1992). Illness cognition: using common sense to understand treatment adherence and affect cognition interactions. Cognitive Therapy and Research, 16 (2), 143-163.

Leventhal, H., Benjamin, Y., Brownlee, S., Diefenbach, M., Leventhal, EA., PatrickMiller, L. et al. (1997). Illness representations: theoretical foundations. In K.J. Petrie \& J.A. Weinman (Eds.) Perceptions of health and illness (pp.1947). Amsterdam: Harwood.

Levy, B.R. (1996). Improving memory in old age by implicit self-stereotyping. Journal of Personality and Social Psychology, 71 (6), 1092-1107.

Levy, B.R. (2003). Mind matters: Cognitive and physical effects of aging selfstereotypes. Journals of Gerontology. Series B: Psychological Sciences and Social Sciences, 58B (4), 203-211.

Levy, B.R. \& Banaji, M.R. (2002). Implicit Ageism. In T.D. Nelson. (Ed.) Stereotyping and prejudice against older persons (pp. 49-75). London: MIT Press.

Levy, B.R., Slade, M.D., Kunkel, S.R. \& Kasl, S.V. (2002). Longevity increased by positive self-perceptions of ageing. Journal of Personality and Social Psychology, 83 (2), 261-270.

Lindesay, J., Briggs, K. \& Murphy, E. (1989). The Guy’s/Age Concern survey. Prevalence rates of cognitive impairment, depression and anxiety in an urban elderly community. British Journal of Psychiatry, 155, 317-329. 
Lobban, F., Barrowclough, C. \& Jones, S. (2003). A review of the role of illness models in severe mental illness. Clinical Psychology Review, 23 (2), 171 196.

Lundervold, D.A. \& Young, L.G. (1992). Older adults' attitudes and knowledge regarding the use of mental health services. Journal of Clinical and Experimental Gerontology, 14 (1), 45-55.

McLoughlin, M., Armstrong, P., Byrne, M., Heaney, D., O’Brien, N. \& Murphy, A. W. (2005). A comparative study on attitudes, mental health, and job stress amongst GPs participating, or not, in a rural out-of hours co-operative. Family Practice, 22 (3), 275-279.

McMurdo, M.E., Witham, M.D. \& Gillespie, N.D. (2005). Including older people in clinical research. British Medical Journal, 331 (7524), 1036-1037.

Mental Health Foundation \& Age Concern. (2006). Promoting mental health and well-being in later life. A first report from the UK Inquiry into Mental Health and Well-Being in Later Life. London: Age Concern (England) \& The Mental Health Foundation. (http://www.mhilli.org)

Millward, L. (2006). Focus Groups. In G.M. Breakwell, S. Hammond, C. FifeSchaw \& J.A. Smith (Eds.) Research Methods in Psychology (pp. 274-298). London: Sage Publications Ltd.

Millward Brown, IMS. (2003). Attitudes towards mental illness. Dublin: Millward Brown. (http://www.mentalhealthireland.ie/news/FinalReport).

Minicuci, N., Maggi, S., Pavan, M., Enzi, G. \& Crepaldi, G. (2002). Prevalence rate and correlates of depressive symptoms in older individuals: the Veneto Study. The Journals of Gerontology Series A: Biological Sciences and Medical Sciences 57, M155-M161. 
Ogden, J. (2000). Health Psychology: A Textbook ( $2^{\text {nd }}$ edn). Buckingham: Open University Press.

Palmore, E.B. (2004). The future of ageism. Issue Brief (March-April). New York: International Longevity Center.

Parkinson, J. (2006). Measuring positive mental health: developing a new scale. Scottish Executive Briefing Paper.

(http://157.203.43.151/uploads/documents/3046-measuring\%20mental\%20wellbeing $\% 20 \% 20$ Affectometer $\% 202 \% 20-\% 20$ WEMWBS $\% 20$ briefing.pdf).

Parnell, V., Worthington, M., Nursing, R. \& Bender, M. (2001). My body is ugly because it is old: ageism and its internalisation. PSIGE Newsletter, 74, 1218.

Paykel, E.S., Tylee, A., Wright, A. et al. (1997). The Defeat Depression Campaign: psychiatry in the public arena. American Journal of Psychiatry, 154 (suppl. 6), 59-65.

Pepin, R. (2006). Intrinsic and extrinsic barriers to mental health care in younger and older adults. Unpublished masters dissertation. University of Colorado: Colorado Springs.

Pinquart, M., Duberstein, P.R. \& Lyness, J.M. (2006). Treatments for late life depressive conditions: a meta-analytic comparison of pharmacotherapy and psychotherapy. American Journal of Psychiatry, 163 (9), 1493-1501.

Podsakoff, P.M., Mackenzie, S.B., Lee, J-Y. \& Podsakoff, N.P. (2003). Common method biases in behavioural research: a critical review of the literature and recommended remedies. Journal of Applied Psychology, 88 (5), 879-903. 
Powell, R.A. \& Single, H.M. (1996). Focus Groups. International Journal for Quality in Health Care, 8 (5), 499-504.

Power, M.J., Quinn, K., Schmidt, S. \& the WHOQOL-OLD Group. (2005). Development of the WHOQOL-OLD module. Quality of Life Research, 14 (10), 2197-2214.

Prohaska, T.R., Leventhal, E.A., Leventhal, H. \& Keller, M.L. (1985). Health practices and illness cognition in young, middle aged, and elderly adults. Journal of Gerontology, 40 (5), 569-578.

Rabins, P. (1996). Barriers to diagnosis and treatment of depression in elderly patients. American Journal of Geriatric Psychiatry, 4 (4), 79-84.

Registrar General for Scotland. (2006). Life Expectancy for Administrative Areas within Scotland, 2003-2005. Crown Copyright.

Resnick, R. (2003). To prescribe or not to prescribe - is that the question? The Psychologist, 16 (4), 184-186.

Ritchie, J. \& Spencer, L. (1994). Qualitative data analysis for applied policy research. In A. Bryman \& R. Burgess (Eds.) Analysing qualitative data (pp. 173-194). London: Routledge.

Robb, C., Haley, W.E., Becker, L.A., Polivka, L.A. \& Chwa, H.J. (2003). Attitudes towards mental health care in younger and older adults: similarities and differences. Aging and Mental Health, 7 (2), 142-153.

Rokke, P.D. \& Scogin, F. (1995). Depression treatment preferences in younger and older adults. Journal of Clinical Geropsychology, 1 (3), 243-257. 
Ron, P. (2007). Elderly people's attitudes and perceptions of aging and old age: the role of cognitive dissonance? International Journal of Geriatric Psychiatry, 22 (7), 656-662.

Rowe, J.W. \& Kahn, R.L. (1997). Successful aging. The Gerontologist, 37 (4), 433440.

Rowe, J.W. \& Kahn, R.L. (1998). Successful aging. New York: Pantheon Books.

Sarkisian, C.A., Hays, R.D., \& Mangione, C.M. (2002). Do older adults expect to age successfully? The association between expectations regarding aging and beliefs regarding healthcare seeking among older adults. Journal of the American Geriatrics Society, 50 (11), 1837-1843.

Sarkisian, C.A., Lee-Henderson, M.H. \& Mangione, C.M. (2003). Do depressed older adults who attribute depression to "old age" believe it is important to seek care? Journal of General Internal Medicine, 18 (12), 1001-1005.

Sartorius, N. (2003). Introduction: Stigma and discrimination against older people with mental disorders. International Journal of Geriatric Psychiatry, 18 (8), 669.

Scheff, T.J. (1966). Being mentally ill. A sociological theory. Chicago: Aldine.

Scogin, F. \& McElreath, L. (1994). Efficacy of psychosocial treatments for geriatric depression: A quantitative review. Journal of Consulting and Clinical Psychology, 62 (1), 69-74.

Scogin, F., Welsh, D., Hanson, A., Stump, J. \& Coates, A. (2005). Evidence-based psychotherapies for depression in older adults. Clinical Psychology: Science and Practice, 12 (3), 222-237. 
Scottish Executive. (2000). Our National Health: A Plan for Action, A Plan for Change. Edinburgh: Crown Copyright.

Scottish Executive. (2002a). Adding Life to Years. Report of the Expert Group on Healthcare of Older People. Edinburgh: Crown Copyright.

Scottish Executive. (2002b). Well? What do you think? A national Scottish survey of public attitudes to mental health, well-being, and mental health problems. Edinburgh: Crown Copyright.

Scottish Executive. (2003). Improving Health in Scotland: The Challenge. Edinburgh: Crown Copyright.

Scottish Executive. (2003-2006). The National Programme for Improving Mental Health and Well-Being. Edinburgh: Crown Copyright. (http://www.wellscotland.info/mentalhealth/national-programme.html).

Scottish Executive. (2004). Well? What do you think? The second national Scottish survey of public attitudes to mental health, well-being, and mental health problems. Edinburgh: Crown Copyright.

Scottish Executive. (2005). The Scottish Health Survey 2003. Edinburgh: Crown Copyright.

Scottish Executive. (2006). Delivering for Mental Health. Edinburgh: Crown Copyright.

Scottish Executive. (2007). All Our Futures: Planning for a Scotland with an Ageing Population. Edinburgh: Crown Copyright. 
Seedsman, T. (2005). 'Successful ageing': A case for optimism. Symposium on Successful Ageing, Ben-Gurion University of the Negev-Israel. Retrieved January 2007 from: (http://www.staff.vu.edu.au/aura/).

Segal, D.L., Coolidge, F.L., Mincic, M.S. \& O'Riley. A. (2005). Beliefs about mental illness and willingness to seek help: a cross-sectional study. Aging and Mental Health, 9 (4), 363-367.

Seymour, J. (2006). Commentary on late-life depression: the differences between early- and late-onset illness in a community based sample. CNS Forum. Retrieved December 2006 from: (http://www.cnsforum.com/).

Smith, J.A., Jarman, M. \& Osborn, M. (1999). Doing interpretative phenomenological analysis. In M. Murray \& K. Chamberlain (Eds.) Qualitative Health Psychology: Theories and Methods (pp. 218-241). London: Sage.

Snowdon, J. (1990). The prevalence of depression in old age. Editorial. International Journal of Geriatric Psychiatry, 5 (3), 141-144.

Tabachnik, B.G. \& Fidell, L.S. (2001). Using multivariate statistics $\left(4^{\text {th }}\right.$ edn). Needham Heights MA: Allyn \& Bacon.

Teachman, B.A. (2006). Aging and negative affect: The rise and fall and rise of anxiety and depression symptoms. Psychology and Aging, 21 (1), 201-207.

United Nations Population Division (1998). World Population Prospects:, The 1998 Revision, Volume II: Sex and Age. New York: United Nations.

United Nations Population Division. (2001). World Population Ageing: 19502050. New York: United Nations. 
United Nations Population Division. (2006). World Population Prospects: The 2006 Revision. New York: United Nations.

U.S. Department of Health and Human Services. (1999). Mental health: A report of The Surgeon General - Executive summary. Rockville, MD: DHHS.

Vaillant, G.E. (2002). Aging Well. London: Little, Brown and Company.

Vaillant, G.E. \& Mukamal, K. (2001). Successful aging. American Journal of Psychiatry, 158 (6), 839-847.

Walker, A. \& Maltby, T. (1997). Ageing Europe. Buckingham: Open University Press.

Waxman, H.M., Carner, E.A. \& Klein, M. (1984). Underutilisation of mental health professionals by community elderly. Gerontologist, 24, 23-30.

Weissman, M.M., Leaf, P.J., Tischler, G.L., Blazer, D.G., Karno, M., Bruce, M.L. et al. (1988). Affective disorders in five United States communities. Psychological Medicine, 18 (3), 141-153.

Wilkinson, S. (2003). Focus groups. In J.A. Smith (Ed.) Qualitative psychology: $a$ practical guide to research methods (pp.184-204). London: Sage Publications Ltd.

Williamson, J., Stokoe, I.H., Gray, S., Fisher, M., Smith, A., Mchee, A. et al. (1964). Old people at home: their unreported needs. Lancet, 283 (7343), 1117-1120.

Willig, C. (2001). Introducing qualitative research in psychology: Adventures in theory and method. Berkshire: Open University Press. 
Winer, B.J. (1971). Statistical principles in experimental design $\left(2^{\text {nd }}\right.$ edn). New York: McGraw-Hill.

Wood, R. \& Bain, M. (2001). The health and well-being of older people in Scotland: insights from national data. Edinburgh: Information and Statistics Division, NHS Scotland.

Woods, R.T. (1999). Mental health problems in late life. In R.T. Woods (Ed.) Psychological problems of ageing: Assessment, treatment and care (pp. 73110). Chichester: John Wiley \& Sons, Ltd.

World Health Organisation (WHO) (2002). Active Ageing: A Policy Framework. Second United Nations World Assembly on Ageing. Madrid:WHO.

World Health Organisation (WHO) \& The World Psychiatric Association (WPA) (2002). Reducing stigma and discrimination against older people with mental disorders: A technical consensus statement. Geneva: WHO.

Yardley, L. (2000). Dilemmas in qualitative health research. Psychology and Health, 15 (2),215-228.

Xavier, F.M.F., Ferraza, M.P.T., Argimon, I., Trentini, C.M., Poyares, D., Bertollucci, P.H. et al. (2002). The DSM-IV 'minor depression' disorder in the oldest-old: prevalence rate, sleep patterns, memory function and quality of life in elderly people of Italian descent in Southern Brazil. International Journal of Geriatric Psychiatry, 17 (2), 107-116.

Zeiss, A. \& Steffen, A. (1996). Treatment issues with elderly clients. Cognitive and Behavioural Practice, 3 (2), 371-390. 


\section{6 - APPENDICES}


12 October 2006

Ms

Trainee Clinical Psychologist
Teiephone:

Facsimile:

Ref:

Dear Ms

Full title of study:

REC reference number:
A comparison of attitudes to mental ill-health in healthy older people and those attending mental health services.

Thank you for your letter of 28 August 2006, responding to the Committee's request for further information on the above research and submitting revised documentation.

The further information was considered at the meeting of the Committee held on 03 October 2006. A list of the members who were present at the meeting is attached.

\section{Confirmation of ethical opinion}

On behalf of the Committee, I am pleased to confirm a favourable ethical opinion for the above research on the basis described in the application form, protocol and supporting documentation as revised.

\section{Ethical review of research sites}

The Committee has not yet been notified of the outcome of any site-specific assessment (SSA) for the research site(s) taking part in this study. The favourable opinion does not therefore apply to any site at present. I will write to you again as soon as one Local Research Ethics Committee has notified the outcome of a SSA. In the meantime no study procedures should be initiated at sites requiring SSA.

\section{Conditions of approval}

The favourable opinion is given provided that you comply with the conditions set out in the attached document. You are advised to study the conditions carefully. 


\section{Approved documents}

The final list of documents reviewed and approved by the Committee is as follows:

\begin{tabular}{|c|c|c|}
\hline Document & Version & Date \\
\hline \multicolumn{3}{|l|}{ Application } \\
\hline Application & 1.0 & 03 July 2006 \\
\hline Investigator CV & 1.0 & \\
\hline Protocol & 6.0 & 03 July 2006 \\
\hline Covering Letter & & 28 August 2006 \\
\hline Covering Letter & & 05 July 2006 \\
\hline Letter from Sponsor & & 30 June 2006 \\
\hline Compensation Arrangements & & 28 August 2006 \\
\hline Compensation Arrangements & & 22 August 2005 \\
\hline $\begin{array}{l}\text { Questionnaire: Attitudes to Mental III-Health } \\
\text { Questionnaire }\end{array}$ & $\begin{array}{l}\text { version } 5 \text { - } \\
\text { Submission } \\
2\end{array}$ & 03 July 2006 \\
\hline Questionnaire: Non-Validated Questionnaire & 5.0 & 03 July 2006 \\
\hline \multicolumn{3}{|l|}{ Questionnaire: Validated Questionnaire } \\
\hline Letter of invitation to participant & 5.0 & 03 July 2006 \\
\hline Letter of invitation to participant & 6 & 23 August 2006 \\
\hline $\begin{array}{l}\text { Participant Information Sheet: Information for } \\
\text { Prospective Participants }\end{array}$ & 6 & 23 August 2006 \\
\hline Participant Information Sheet: Information Sheet & 5.0 & 03 July 2006 \\
\hline Participant Consent Form: Informed Consent Form & 1.0 & 03 July 2006 \\
\hline $\begin{array}{l}\text { Participant Consent Form: Informed Consent - Older } \\
\text { peoples' attitudes to mental ill-health: Focus Group }\end{array}$ & 3 & 03 July 2006 \\
\hline $\begin{array}{l}\text { Participant Consent Form: Informed Consent - Older } \\
\text { peoples' attitudes to mental ill-health }\end{array}$ & 2 & 23 August 2006 \\
\hline \multicolumn{3}{|l|}{ Response to Request for Further Information } \\
\hline Questionnaire cover letter & 3 & 23 August 2006 \\
\hline Focus Group informed Consent Form & 1.0 & 03 July 2006 \\
\hline Focus Group Information Sheet & 2.0 & 03 July 2006 \\
\hline $\begin{array}{l}\text { Research Study: Older Peoples' Attitudes to Mental III- } \\
\text { health- Letter }\end{array}$ & 2.0 & 03 July 2006 \\
\hline
\end{tabular}

\section{Research governance approval}

The study should not commence at any NHS site until the local Principal Investigator has obtained final research governance approval from the R\&D Department for the relevant NHS care organisation. 


\section{Statement of compliance}

The Committee is constituted in accordance with the Governance Arrangements for Research Ethics Committees (July 2001) and complies fully with the Standard Operating Procedures for Research Ethics Committees in the UK.

With the Committee's best wishes for the success of this project

Yours sincerely

Email: fiona.thow@faht.scot.nhs.uk

Enclosures:

List of names and professions of members who were present at the meeting

Standard approval conditions SL-AC2

Site approval form

Copy to:

University of Edinburgh School of Health in Social Science, 


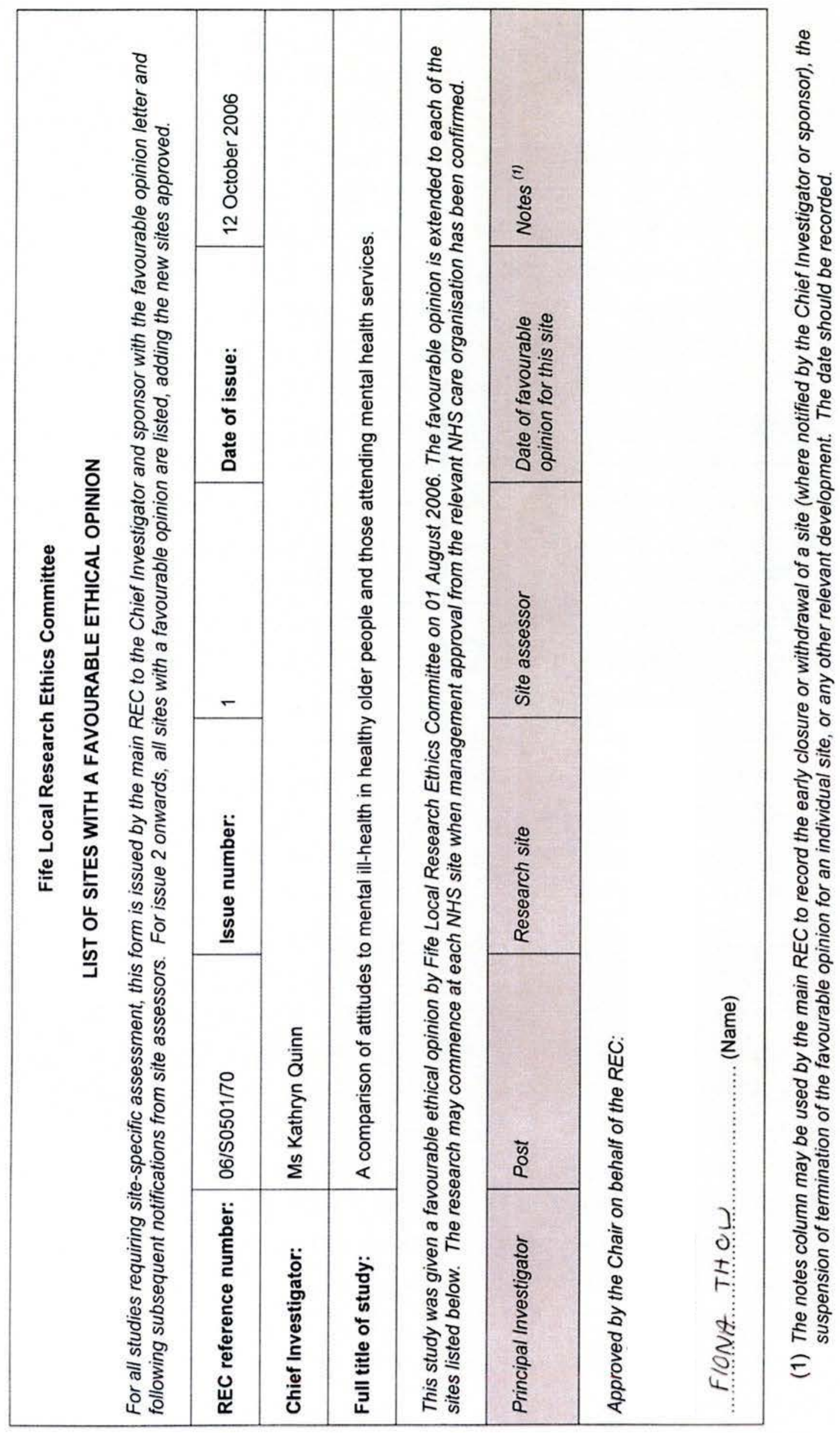




\section{APPENDIX 1.2: NHS R \& D APPROVAL}

\begin{tabular}{|c|c|c|c|}
\hline Ms & & Date & 6 October 2006 \\
\hline Trainee Clinical Psychologist & & Your Ref & \\
\hline Dept of Clinical Psychology & & Our Ref & \\
\hline Hosnital & & Enquiries to & \\
\hline & (5) $[F \quad$ if & Frxtension & \\
\hline & & Fax No & \\
\hline & $135 \ldots$ & EImail & \\
\hline ear Ms & $\ldots \ldots \ldots \ldots$ & & \\
\hline
\end{tabular}

Project Title: "A comparison of attitudes to mental ill-health in healthy older people and those attending mental health services"

Thank you for your application to carry out the above project.

Your project documentation has been reviewed for resource and financial implications for NHS Primary Care Division and I am happy to inform you that Management Approval has been granted, subject to all necessary Ethical approvals and honorary contracts being in place.

Details of our participation in this study will be included in quarterly returns to the National Research Register and annual returns we are expected to complete as part of our agreement with the Chief Scientist Office. The enclosed Research Registration Form has been prepared and should be checked, signed and returned together with the attached NRR Form (in the enclosed envelope) to the R\&D Office, Hospital, or need further information contact or at If you have any questions

May I take this opportunity to remind you that all research undertaken in NHS is managed strictly in accordance with the Research Governance Framework for Health \& Community Care (http://www.show.scot.nhs.uk/cso/) and that all research should be carried out according to Good Clinical Practice (GCP). In order to comply with the RGF, the R\&D Office are required to hold copies of all study protocols, ethical approvals and amendments for the duration of this study.

You will also be required to provide information in regard to monitoring and study outcomes, including a lay summary on completion of the research. I would like to wish you every success with your study and look forward to receiving a summary of the findings for dissemination once the project is complete.

Yours sincerely

Dr

Medical Director, Primary Care

Cc: 


\section{APPENDIX 1.3: SUMMARY OF AMENDMENTS REQUESTED BY ETHICS COMMITTEE}

Several minor changes were requested to the original design, most notably for the researcher to be able to identify and retrospectively contact research participants in the event that their responses to certain items in the questionnaire uncovered previously unidentified mental health difficulties. All questionnaires were therefore coded with a link to participant's contact details (held separately from questionnaire data), on the assumption that participants agreed to provide this information. Contact data were required to be held separately from questionnaire data and to be used only to identify individuals at risk or to provide a summary copy of study results to agreeable participants. One further recommendation related to the consideration of implicit study consent on the return of completed questionnaires. Finally, the Committee requested to be informed of any additional major changes to the questionnaire as a result of piloting undertaken through focus groups as outlined in the study design. No major changes were identified (see Section 2.4.3). 


\section{APPENDIX 2.1: STUDY INVITATION LETTER}

Clinical Psychology Department

[date]

Dear Sir/Madam

\section{• Older Peoples' Attitudes to Mental Ill-health •}

Thank you very much for expressing an interest in this research study. This study is being undertaken in part contribution towards a doctorate degree in clinical psychology in conjunction with the [...] and NHS [...]. The study aims to collect information about the views of older adults to mental ill-health, and also asks about attitudes to growing older and subjective well-being.

\section{- What does participation involve and why have I been chosen?}

Participation involves completing a series of short questionnaires. You have been approached to take part in this study because you are aged 65 or over and are either a member of [...] User Group Panels/[...] Elderly Forum, or are currently (or have recently been) a service-user. An information leaflet is enclosed outlining the steps of participation and what taking part will involve for you.

\section{$\bullet$ Why should you participate?}

Your participation in the project is very important. Older people are a population often underserved by the mental health system. Your responses will provide valuable information about the views and needs of older adults in our society in relation to mental ill-health, stigma and discrimination. 


\section{-What to do now?}

\section{If you wish to take part or would like to know more:}

Please read through the attached information sheet and decide if you wish to take part. If you do wish to participate please inform the person who gave you this letter or contact the study researcher directly (please return the attached request slip including your name and address or contact the researcher by phone or email). You will then be asked to complete an informed consent form. Following this, if you meet with study inclusion criteria, you will be provided with the questionnaires for completion. These can be provided to you in person or you can opt to receive these through the post. Once received, questionnaires can also be returned by post in the pre-paid envelope provided. You may withdraw from the study at any time, even if you have previously agreed to take part, and without implication.

If you would like further information before you decide if you want to take part, please contact the study researcher who will be very pleased to talk to you.

\section{If you do not wish to take part:}

Thank you very much for taking the time to read through this letter. You need do nothing more. Your decision not to take part in this study is completely respected.

Whatever you decide, should you have any queries about any aspect of the study please do not hesitate to contact the study researcher. Contact details are as follows:

(Study Researcher) (Academic Supervisor)

Thank you very much for taking the time to read about this study. Yours faithfully

Trainee Clinical Psychologist

Enc. 


\section{Older Peoples' Attitudes to Mental III-health \\ Information for Prospective Participants}

Thank you for reading this.

\section{$\bullet$ Introduction}

You are being invited to take part in a research study about the views of older people toward mental ill-health. This study is being undertaken for educational purposes and is in part contribution towards a doctorate degree in clinical psychology in conjunction with the [...] and NHS [...]. Before you decide if you would like to participate it is important that you know a bit more about the study and what participation will involve. Please take time to read the following information carefully and raise any questions you may have with the study researcher (contact details are provided at the end of this sheet). Please ask if there is anything you are unclear about or if you would like more information.

\section{-What is the study about?}

Our attitudes towards things can often influence how we view ourselves and other people, and how we react to events and experiences. In our society there is prevalence of negative attitudes about mental ill-health and ageing which can lead to stigma and discrimination, and may result in some older people failing to seek treatment for mental health problems if they occur. So far, however, little research has been carried out to assess the views of older people in this area. The aim of this study is to find out what people aged 65 and over think about mental ill-health generally, and about mental ill-health as it affects older people.

\section{-Why have I been chosen?}

Two groups of people are being asked to participate in the study: those who are currently attending (or have very recently attended) mental health services for common problems such as depression and anxiety, and those who are not. Having two groups will allow us to compare the attitudes of both, to see if there is a difference. You have been invited to participate because, at the moment, you fit one of these categories and you are also aged 65 or over. 
Participation in this study is entirely voluntary. If you decide that you would like to take part you will be given this information sheet to keep and will be asked to sign a consent form. If you initially decide to take part and later wish to withdraw, you can do so at any time and without giving a reason. Your decision to withdraw will be respected and will not affect any current or future care you may receive.

\section{What will participation involve?}

If you agree to take part and you meet the inclusion criteria for the study, you will be asked to provide some general demographic information (e.g. age and gender) and to complete five short questionnaires about attitudes to ageing and mental ill-health, and about health behaviours and subjective well-being. Your responses to all of these will be entirely confidential, and should take approximately $30-40$ minutes to complete. You will only be asked to complete the questionnaires once.

\section{-What are the possible benefits of taking part?}

There is no direct benefit to you of taking part. However, the information you provide will be extremely valuable in helping us to find out whether older adults hold attitudes to mental ill-health which prevent them from obtaining help and/or lead to reductions in quality of life and well-being.

\section{Will my participation in the study be kept confidential?}

The information you provide will be kept strictly confidential. You will not be personally identified in any of the study results or reports. The only person with direct access to your information will be the study researcher. All of the information held by the researcher will be held on a protected database.

Please note: There may be times when we are concerned about a participant's responses to some questionnaire items (for example, if the answers to some questions suggest the possibility of mental health difficulties). In the unlikely event that this occurs, we would like to be able to contact you to discuss your results with you personally and confidentially, and to offer you some support and advice either directly over the telephone, by sending you some more information, or by recommending that you contact your General Practitioner in the first instance. In order for us to do this, please provide us with your name and address when you return the questionnaires. You may also wish to provide your name and address if you wish to receive a copy of the study results. Your contact details will remain strictly confidential and will not be made known to anyone outwith the research team. 


\section{-What will happen to the results of the study?}

The results will be included in a doctoral thesis submitted to the [...] by the study researcher. You will not be identified in this, or in any other report resulting from the study. A brief summary of the results will be made available to all those who participated in the study, if requested. You will not be identified in this summary.

\section{-Who else knows about the study?}

The study has been reviewed by [...] Local Research Ethics Committee, and by the [...] Doctorate in Clinical Psychology Course Organisation Committee.

\section{-What should I do now?}

If you wish to take part: please inform the person who gave you this letter or contact the study researcher directly (as outlined in the covering letter). You will be provided with an informed consent form for completion. Following this, assuming you meet the study inclusion criteria, you will then receive the questionnaires.

If you do not wish to take part: we would like to thank you for taking the time to read through this information sheet. You need do nothing more. Your decision not to take part is completely respected.

If you have any queries about any aspect of the study or require further information, please do not hesitate to contact us at the address given below:

Study Researcher

Research Supervisor

Clinical Psychology Department

Telephone:

Telephone: Email:

Email:

If you wish to take part you will be given a copy of this information sheet to keep.

Thank you for taking the time to read this letter. 


\section{Informed Consent Form}

\section{Older peoples' attitudes to mental ill-health \\ Principal Researcher:}

Please tick box

- I confirm that I have read and understand the information sheet dated 23 August, 2006 (Version: 6) for the above study. I have had the opportunity to consider the information, ask questions, and have had these answered satisfactorily.

- I understand that my participation is voluntary and that I am free to withdraw at any time, without giving any reason, without my medical care or legal rights being affected.

- I understand that the information I provide during this study may be looked at by responsible individuals from NHS $[\ldots] /[\ldots]$, where it is relevant to my taking part in this research. I give permission for these individuals to have access to my information.

- I understand that in the event of my questionnaire responses giving cause for concern about my well-being, I can be contacted directly by the study researcher to discuss various options regarding how to obtain further support.

- I agree to take part in the above study. researcher)

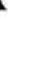




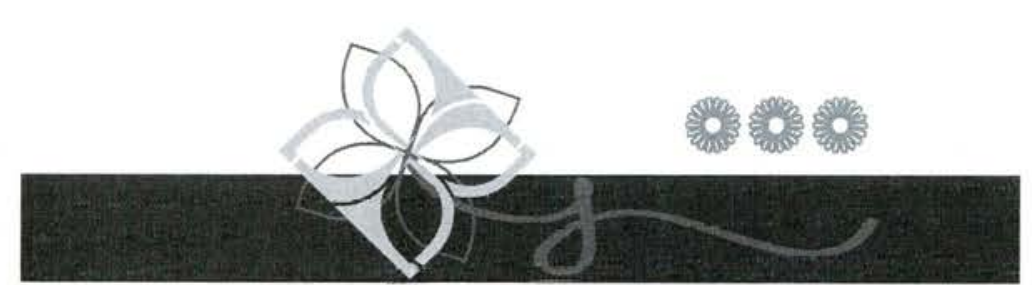

Attitudes to Mental Ill-Health Questionnaire

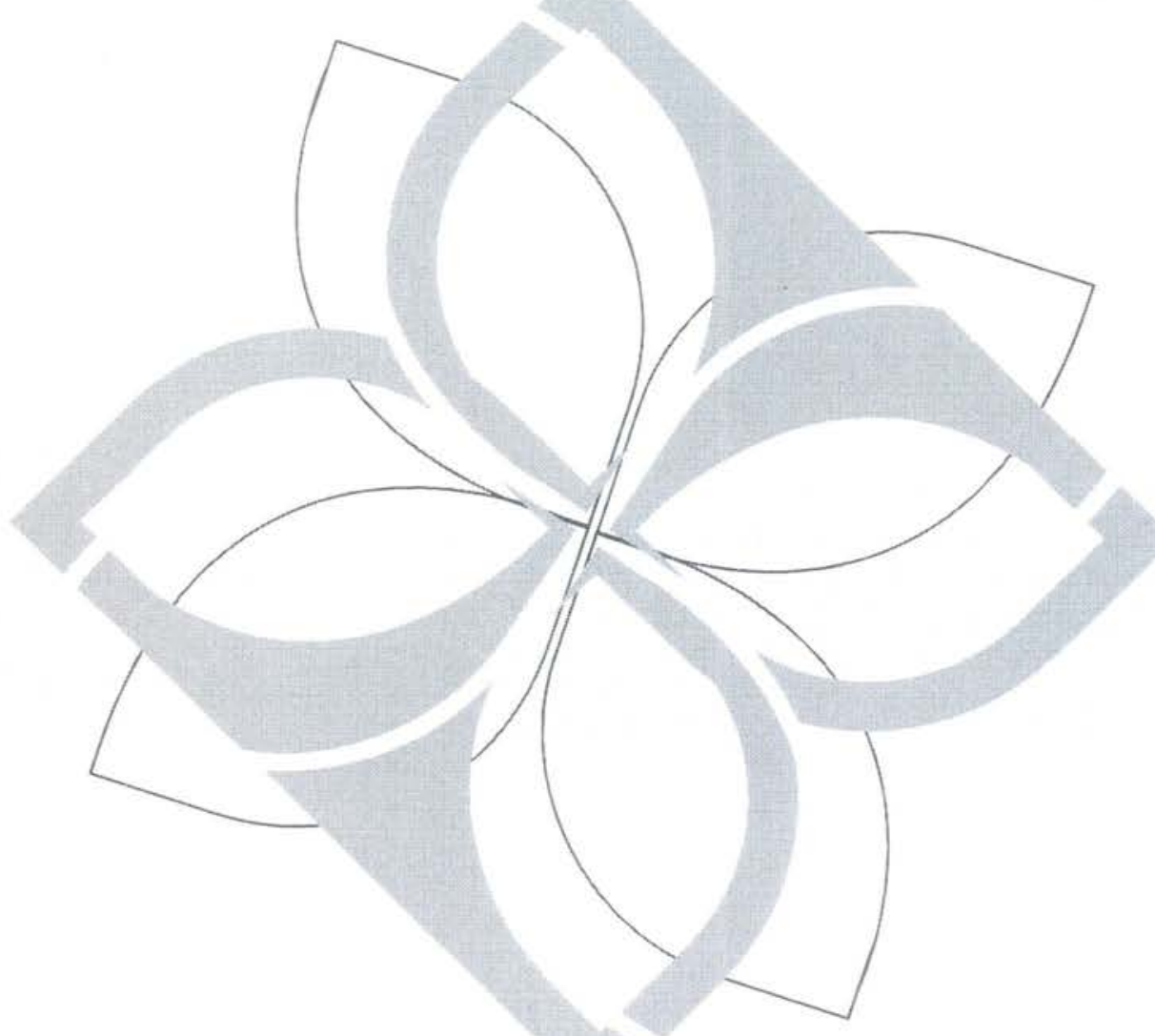

184 


\section{Older Peoples' Attitudes to Mental Ill-Health}

\section{Participant Contact Details}

As indicated in the covering letter, please provide your contact details below:

Name:

Address:

Telephone:

Email:

Please indicate below whether you would like us to send you a copy of the study results:

Yes, I would like to receive a copy of the study results.

No, I do not wish to receive a copy of the study results.

THANK YOU. PLEASE NOW CONTINUE TO COMPLETE THE QUESTIONNAIRES. 


\section{PART 1 : SOCIODEMOGRAPHIC QUESTIONS} SOCIO-DEMOGRAPHIC INFORMATION

\section{ABOUT YOU}

Before you begin completing the questionnaires we would like to ask you a few general questions about yourself:

You have been approached to take part in this research because you are either a member of Age Concern Fife User Group Panels/Fife Elderly Forum or are currently attending (or have recently attended) mental health services (i.e. psychological and psychiatric services). Please indicate which of the following statements applies to you by placing a tick in the appropriate box:

I I am a member of Fife User Group Panels/Fife Elderly Forum.

I I am a current user of mental health services (or have recently been discharged - i.e. in the last six months).

I I am both a member of the User Panels and a current user of mental health services.

1. What is your gender?

Please tick as appropriate
Male
Female

2. What is your age?

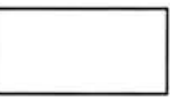

3. What is your current marital status?

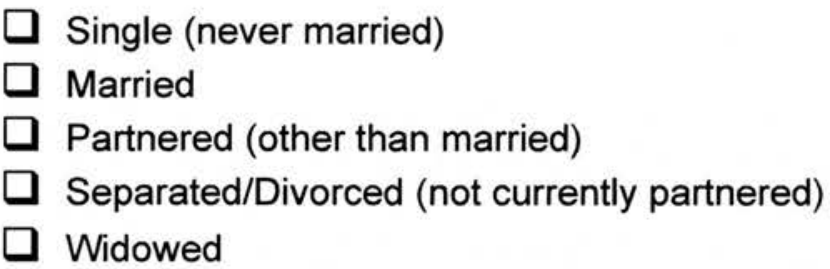

4. What is your highest of educational attainment?
Primary school
$\square$ High school
- Trade or technical certificate
- College diploma or degree
$\square$ University degree
$\square$ Other: Please Specify 
5. What is/was your occupation?

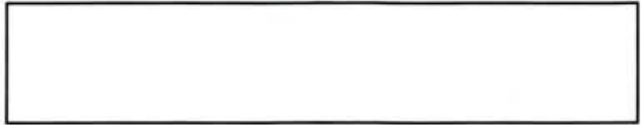

6. Living arrangements:

Please tick one response as appropriate

$\square$ Living at home (supported by family/carers or partner)

$\square$ Living with family but not in own home

$\square$ Living at home (unsupported by family/carer or partner)

$\square$ Living in residential care

$\square$ Living in sheltered housing/community care

$\square$ Living in nursing home

$\square$ Living in a long-stay patient ward (hospital)

$\square$ Other (please give details below)

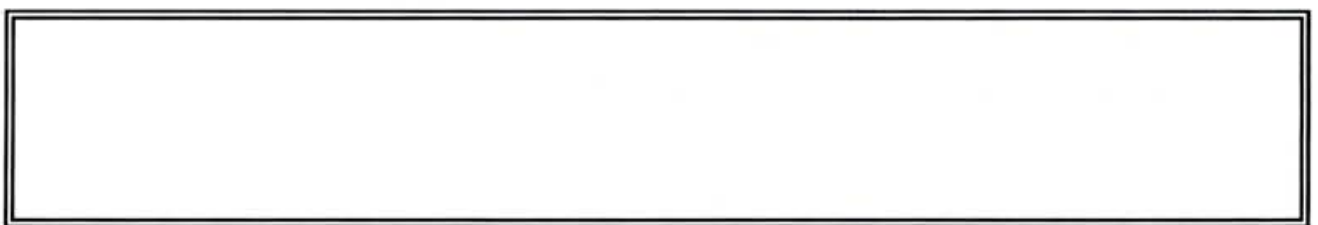

7. Over the last twelve months would you say your health has on the whole been:
$\square$ Good
- Fairly Good
$\square$ Not good

8. Please provide details of any medical conditions which impact on your quality of life. Please also list any prescribed medications (if known):

\begin{tabular}{|l|l|}
\hline Medical Condition(s) & Prescribed Medication \\
\hline & \\
& \\
\hline
\end{tabular}


9. In the past have you or a close member of your family/significant other ever experienced mental health problems (such as anxiety or depression) for which you have attended mental health services (e.g. psychology, psychiatry, community psychiatric nurse etc)?

Please tick one option only

$\square$ Yes, in the past I have previously attended mental health services.

$\square$ Yes, a close member of my family/ a significant other has previously attended mental health services.

$\square$ Yes, both I and a close member of my family/significant other have previously attended mental health services.

$\square$ No, I have no prior experience of attending mental health services (either directly or indirectly via a family member/significant other).

Thank you. Please now continue with the rest of the questionnaire. 


\section{PART 2: ATTITUDES TO MENTAL ILL-HEALTH QUESTIONNAIRE \\ PART A}

nstructions: This questionnaire asks for your views about mental health problems. Please indicate how much rou agree or disagree with the following statements by circling the appropriate response:

$\begin{array}{cccccc}\begin{array}{c}\text { Strongly } \\ \text { agree }\end{array} & \begin{array}{c}\text { Tend to } \\ \text { agree }\end{array} & \begin{array}{c}\text { Neither } \\ \text { agree nor } \\ \text { disagree }\end{array} & \begin{array}{c}\text { Tend to } \\ \text { disagree }\end{array} & \begin{array}{c}\text { Strongly } \\ \text { disagree }\end{array} & \begin{array}{c}\text { Don't } \\ \text { Know }\end{array} \\ & & \text { ang } & \end{array}$

f I was suffering from mental realth problems, I wouldn't want seople knowing about it

The public should be better orotected from people with nental health problems

Anyone can suffer from mental tealth problems

would find it hard to talk to omeone with mental health roblems

'eople are generally caring and ympathetic to people with rental health problems

'eople with mental health iroblems are often dangerous

The majority of people with rental health problems recover

'eople with mental health roblems should have the same ights as anyone else 


\section{Part B}

Instructions: Listed below are potential reasons why people do not seek out psychological therapy (counselling). Please read each carefully, and rate to what extent you agree or disagree with the following statements. Please circle one answer for each item, even if you are unsure.

1. I would see a psychologist (counsellor) because psychologists enjoy working with someone my age.

$\begin{array}{ccccc}1 & 2 & 3 & 4 & 5 \\ \text { Strongly } & \text { Disagree } & \text { Neither Agree } & \text { Agree } & \text { Strongly } \\ \text { Disagree } & & \text { or Disagree } & & \text { Agree }\end{array}$

2. I would not go to a psychologist because I would feel shame.
1
Strongly
Disagree
2
Disagree
3
Neither Agree
or Disagree
4
5
Agree Strongly
Agree

3. I would not see a psychologist because psychologists think working with someone my age is a waste of time.
1
Strongly
Disagree
2
3
Disagree $\quad \begin{gathered}\text { Neither Agree } \\ \text { or Disagree }\end{gathered} \quad$ Agree $\quad \begin{gathered}\text { Strongly } \\ \text { Agree }\end{gathered}$
Disagree $\quad \begin{gathered}\text { Neither Agree } \\ \text { or Disagree }\end{gathered} \quad$ Agree $\quad \begin{gathered}\text { Strongly } \\ \text { Agree }\end{gathered}$
$4 \quad 5$
Disagree $\quad \begin{gathered}\text { Neither Agree } \\ \text { or Disagree }\end{gathered} \quad$ Agree $\quad \begin{gathered}\text { Strongly } \\ \text { Agree }\end{gathered}$

4. I would not go to a psychologist because psychotherapy is for crazy people.

$\begin{array}{ccccc}1 & 2 & 3 & 4 & 5 \\ \text { Strongly } & \text { Disagree } & \text { Neither Agree } & \text { Agree } & \text { Strongly } \\ \text { Disagree } & & \text { or Disagree } & & \text { Agree }\end{array}$

5. I would see a psychologist but psychologists' time is better spent working with younger people.
1
Strongly
2
Disagree
Disagree
3
4
5
Agree Strongly
Neither Agree
or Disagree
Agree

6. I would see a psychologist because normal people go to psychotherapy.
Strongly
2
Disagree
Disagree
3
4
5
Neither Agree Agree Strongly
or Disagree
Agree 
7. I would see a psychologist because psychologists find working with someone my age worthwhile.

$\begin{array}{ccccc}1 & 2 & 3 & 4 & 5 \\ \text { Strongly } & \text { Disagree } & \text { Neither Agree } & \text { Agree } & \text { Strongly } \\ \text { Disagree } & & \text { or Disagree } & & \text { Agree }\end{array}$

8. I would not see a psychologist because it is a sign of weakness.

$\begin{array}{ccccc}1 & 2 & 3 & 4 & 5 \\ \text { Strongly } & \text { Disagree } & \text { Neither Agree } & \text { Agree } & \text { Strongly } \\ \text { Disagree } & & \text { or Disagree } & & \text { Agree }\end{array}$

9. I would not go to a psychologist because people my age are too set in their ways to make changes.

$\begin{array}{ccccc}1 & 2 & 3 & 4 & 5 \\ \text { Strongly } & \text { Disagree } & \text { Neither Agree } & \text { Agree } & \text { Strongly } \\ \text { Disagree } & & \text { or Disagree } & & \text { Agree }\end{array}$

10. I would go to psychotherapy but people my age can't change.

$\begin{array}{ccccc}1 & 2 & 3 & 4 & 5 \\ \text { Strongly } & \text { Disagree } & \begin{array}{c}\text { Neither Agree } \\ \text { or Disagree }\end{array} & \text { Agree } & \begin{array}{c}\text { Strongly } \\ \text { Disagree }\end{array} \\ & & & \text { Agree }\end{array}$

11. I would not go to a psychologist because normal people don't go to psychotherapy.

$\begin{array}{ccccc}1 & 2 & 3 & 4 & 5 \\ \text { Strongly } & \text { Disagree } & \text { Neither Agree } & \text { Agree } & \text { Strongly } \\ \text { Disagree } & & \text { or Disagree } & & \text { Agree }\end{array}$




\section{Part C}

Instructions: Below is a description of depression followed by a list of attitudes or beliefs which people may hold about depression. Please read each statement carefully and circle the number that indicates how much you agree or disagree with the statement, this can often be your first response.

Depression is a prolonged feeling of sadness which can be characterised by the following changes: a loss of enjoyment or interest in normal activities, fatigue of a loss of energy, feelings of worthlessness or guilt, sleep and appetite disturbance, and poor concentration, As time passes a sense of hopelessness can grow and lead to thoughts of suicide.

1. Given the losses and chronic illnesses that older people experience, depression is understandable.

$\begin{array}{lllllllll}\text { Agree Strongly } & 1 & 2 & 3 & 4 & 5 & 6 & 7 & \text { Disagree Strongly }\end{array}$

2. Depression is just a normal part of old age.

Agree Strongly

\begin{tabular}{lllll}
1 & 2 & 3 & 4 & 5 \\
\hline
\end{tabular}

$6 \quad 7$

Disagree Strongly

3. When older people get depressed, there is not much that can be done about it.

Agree Strongly

$$
1
$$

23

$4 \quad 5$

$6 \quad 7$

Disagree Strongly

4. Younger people have little reason to be depressed.

$\begin{array}{lllllllll}\text { Agree Strongly } & 1 & 2 & 3 & 4 & 5 & 6 & 7 & \text { Disagree Strongly }\end{array}$

5. Younger people are not expected to be depressed because they are young.

Agree Strongly

$\begin{array}{llllllll}1 & 2 & 3 & 4 & 5 & 6 & 7 & \text { Disagree Strongly }\end{array}$




\section{PART 3: ATTITUDES TO AGEING QUESTIONNAIRES}

\section{Instructions:}

This questionnaire asks you how you feel about growing older.

Please answer all the questions. If you are unsure about which response to give to a question, please choose the one that appears most appropriate. This can often be your first response.

Please keep in mind your standards, hopes, pleasures and concerns. We ask that you think about your life in general.

For example, thinking how you feel in general, a question might ask:

\section{I dislike growing older}

\begin{tabular}{c|c|c|c|c} 
Not at all true & Slightly true & Moderately true & Very true & Extremely true \\
1 & 2 & 3 & 4 & 5
\end{tabular}

You should circle the number that best fits how true the statements are for you. So you would circle the number 4 if you dislike growing older "Very much", or circle number 1 if you are "Not at all" concerned about growing older. Please read each question, assess your feelings, and circle the number on the scale for each question that gives the best answer for you. 
The following questions ask how much you agree with the following statements. If you agree with the statements an extreme amount circle the number next to "strongly agree". If you do not agree with the statements at all, circle the number next to "Strongly disagree". You should circle one of the numbers in between if you wish to indicate your answer lies somewhere between "Strongly disagree" and "Strongly agree".

1. As people get older they are better able to cope with life.

Strongly disagree

1
Disagree

2
Uncertain

3
Agree

4

\begin{tabular}{c|c} 
Agree & Strongly agree \\
4 & 5
\end{tabular}
Strongly agree

5

2. It is a privilege to grow old.

Strongly disagree

Disagree

2
Uncertain
3

Agree

4

$\underset{4}{\text { Agree }}$

\section{Old age is a time of loneliness.}

Strongly disagree

Disagree

Strongly agree

5

4. Wisdom comes with age.

Strongly disagree

1
Disagree
2
3
Uncertain

Strongly agree

5

\section{There are many pleasant things about growing older.}

Strongly disagree

1
Disagree
2
Uncertain 3
Agree

4

7. It is important to take exercise at any age.

Strongly disagree

1
Disagree

2
Uncertain 3
Agree
Strongly agree

5

6. Old age is a depressing time of life.

Strongly disagree
Disagree

2 \begin{tabular}{c|c} 
Agree & Strongly agree \\
4 & 5
\end{tabular}

\begin{tabular}{c|c} 
Agree & Strongly agree \\
4 & 5
\end{tabular}

\begin{tabular}{c|c} 
Agree & Strongly agree \\
4 & 5
\end{tabular}
Strongly agree 
The following questions ask how true the following statements are for you. If the statement is "Extremely" true for you, circle the number next to "Extremely true". If the statements are not true for you at all, circle the number next to "Not at all true". You should circle one of the numbers in between if you wish to indicate your answer lies somewhere between "Not at all true" and "Extremely true".

8. Growing older has been easier than I thought.

\begin{tabular}{c|c|c|c|c} 
Not at all true & Slightly true & Moderately true & Very true & Extremely true \\
1 & 2 & 3 & 4 & 5
\end{tabular}

9. I find it more difficult to talk about my feelings as I get older.

\begin{tabular}{c|c|c|c|c} 
Not at all true & Slightly true & Moderately true & Very true & Extremely true \\
1 & 2 & 3 & 4 & 5
\end{tabular}

10. I am more accepting of myself as I have grown older.

Not at all true

1
Slightly true

2
Moderately true

3
Very true

4

Very true 4

Very true 4$$
4
$$

Extremely true 5

11. I don't feel old.

Not at all true

$$
\begin{gathered}
\text { Slightly true } \\
2
\end{gathered}
$$$$
3
$$

Extremely true 5

12. I see old age mainly as a time of loss.

Not at all true Slightly true 2 3

Extremely true 5

13. My identity is not defined by my age.

Not at all true 1
Slightly true

2 \begin{tabular}{|l|} 
Moderately true \\
3
\end{tabular}
Very true 4
Extremely true 5

14. I have more energy now than I expected for my age.

Not at all true

1
Slightly true

2
Moderately true

3
Very true 4

Extremely true

5

15. I am losing my physical independence as I get older.

Not at all true 1
Slightly true

2
Moderately true

3
Very true 4
Extremely true 5

16. Problems with my physical health do not hold me back from doing what I want to.

Not at all true

1
Slightly true

2 \begin{tabular}{c|c|c|c|} 
Moderately true \\
3
\end{tabular}
Very true

4
Extremely true 
17. As I get older, I find it more difficult to make new friends.

\begin{tabular}{c|c|c|c|c} 
Not at all true & Slightly true & Moderately true & Very true & Extremely true \\
1 & 2 & 3 & 4 & 5
\end{tabular}

18. It is very important to pass on the benefits of my experiences to younger people.

Not at all true

Slightly true

2
Moderately true

3
Very true

4

Extremely true

5

19. I believe my life has made a difference.

Not at all true

Slightly true

2
Moderately true
3
Very true

4

Extremely true 5

20. I don't feel involved in society now that I am older.

Not at all true

Slightly true

2

\section{Moderately true}

3
Very true 4

21. I want to give a good example to younger people.

Not at all true

1
Slightly true

2
Moderately true

3
Very true 4
Extremely true 5

22. I feel excluded from things because of my age.

Not at all true

1
Slightly true

2
Moderately true

3
Very true

4
Extremely true 5

23. My health is better than I expected for my age.

Not at all true

1

\begin{abstract}
Slightly true
\end{abstract}
2
Moderately true

3
Very true

4

Extremely true 5

Extremely true 5

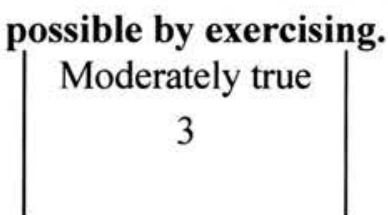

Very true
4
Extremely true 5 
We are interested if you would answer these questions on how you have been feeling over the past few weeks.

Please read each item and circle your first considered response.

1. I am keeping in touch with today's Society.

2. As I got older I became a more efficient worker.

3. As I get older I am becoming more of a burden to my relatives.

4. My moral values are relevant in today's world.

5. My best achievements are in the past.

6. As I get older I move around far less.

7. I feel I am just as capable of engaging in satisfying sexual activity as when I was younger.

8. My health is surprisingly good.

9. As I get older I need someone to keep an eye on me.

10. Hearing loss embarrasses me.

11. As I get older, I find that I tend to get confused more often.
Disagree Disagree

Strongly

Agree

Agree

Strongly

Disagree Disagree Agree Agree

Strongly

Strongly

Disagree

Strongly

Disagree Agree

Agree

Strongly

Disagree

Strongly

Disagree Agree

Agree

Strongly

Disagree

Strongly

Disagree Agree

Agree

Strongly

Disagree

Disagree

Agree

Agree

Strongly

Disagree

Strongly

Disagree

Agree

Agree

Strongly

Disagree

Strongly

Disagree

Agree

Agree

Strongly

Disagree

Strongly

Disagree Agree

Agree

Strongly

Disagree

Strongly

Disagree Agree

Agree

Strongly

Disagree

Strongly
Disagree Agree Agree

Strongly 
12. As I get older my memory is getting worse.

13. My eyesight is definitely failing.

14. I feel that my old age has been full of exciting opportunities.

15. I think that within a few years I shall be in a hospital/home rather than living in my own home.

16. As I've got older I'm less able to practice good table manners.

17. I feel that I am quite capable of learning new skills.

18. I need medicines to keep me going.

19. As I get older I'm getting more forgetful.

20. I move around far less than when I was young.

21. I still have a desire for sex.

22. I have a surprisingly good state of health.

23. As I get old I'm getting more forgetful.
Disagree

Strongly

Disagree

Strongly

Disagree

Strongly

Disagree

Strongly

Disagree Agree

Agree

Strongly

Disagree

Strongly

Disagree

Strongly

Disagree

Strongly

Disagree

Strongly

Disagree

Agree

Agree

Strongly

Disagree Disagree Agree Strongly

Disagree

Strongly

Disagree

Agree

Agree

Disagree

Strongly

Disagree

Agree

Agree

Disagree

Strongly
Strongly

Strongly

Agree

Strongly

Agree

Strongly 


\section{PART 4: WELL-BEING QUESTIONNAIRE}

\section{Instructions:}

We would like to know if you have had any medical complaints and how your health has been in general, over the last few weeks. Please answer ALL the questions by circling the answer which you think most nearly applies to you. Remember that we want to know about present and recent complaints, not those that you had in the past.

It is important that you try to answer ALL the questions. Thank you very much for your co-operation.

\section{Have you recently....}

1. been able to concentrate on whatever you're doing?

2. lost much sleep over worry?

3. felt that you are playing a useful part in things?

4. felt capable of making decisions about things.

5. felt constantly under strain?

6. felt you couldn't overcome your difficulties?

7. been able to enjoy your normal day-to-day activities?

8. been able to face up to your problems?

9. been feeling unhappy and depressed?

10. been losing confidence in yourself?

\section{1. been thinking of yourself as a} worthless person?

12. been feeling reasonably happy all things considered.

$\begin{array}{llll}\begin{array}{l}\text { Better } \\ \text { than usual }\end{array} & \begin{array}{l}\text { Same as } \\ \text { Usual }\end{array} & \begin{array}{l}\text { Less than } \\ \text { usual }\end{array} & \begin{array}{l}\text { Much less } \\ \text { than usual }\end{array}\end{array}$

Not at all No more

Rather more

Much more than usual than usual than usual

$\begin{array}{ll}\text { More so } & \text { Same as } \\ \text { than usual } & \text { usual }\end{array}$

More so Same as

than usual usual

Not at all

No more than usual

Not at all

More so than usual

More so than usual

Same as usual

Not at all

No more than usual

Not at all

No more than usual

Not at all

No more than usual

More so than usual
About same as usual
Less useful than usual

Less so than usual

Rather more than usual

Rather more than usual

Much more than usual

Less so than usual

Much less than usual

Less so than usual

Much less able

Rather more

Much more than usual

\section{than usual}

Rather more

Much more than usual than usual

Rather more Much more than usual than usual

Much less

Less so than usual than usual 
PART 5: HEALTH BEHAVIOUR QUESTIONNAIRE

Instructions: Please respond to the following items by circling the number which most closely corresponds to how frequently you perform each behaviour.

1) I avoid food with additives.

2) I take vitamins.

3) I eat bran or other high fibre foods.

4) I avoid too much physical exertion.

5) I do regular aerobic or strenuous exercise.

6) I have regular medical check-ups.

7) I get enough sleep.

8) I try to breathe clean air and drink pure water.

9) I use remedies from the chemist.

10) I eat a balanced diet.

11) I try to find out what others do to prevent ill-health.

12) I try to get medical information and understand the causes of illhealth.

13) I avoid harmful health habits like smoking and excessive drinking.

14) I avoid salt and heavily salted foods.

\section{Never}

1

2

3

Always

1

1

2

3

5

5 
15) I use laxatives or enemas.

$\begin{array}{lllll}1 & 2 & 3 & 4 & 5 \\ 1 & 2 & 3 & 4 & 5\end{array}$

16) I avoid too much emotional distress.

17) I have friends and maintain a good family life.

18) I avoid feelings like anger, anxiety $1 \quad \begin{array}{lllll}2 & 3 & 4 & 5\end{array}$ and depression.

19) I think positively.

20) I take things as they come and don't struggle.

21) I stay mentally alert and active. $\quad 1 \quad 3 \quad \begin{array}{lllll}3 & 4\end{array}$ 
Thank you very much for completing the questionnaires.

If you have any further comments in relation to this study please indicate below:

THANK YOU. PLEASE NOW RETURN THE QUESTIONNAIRES TO THE STUDY RESEARCHER IN THE PRE-PAID ENVELOPE PROVIDED. 


\section{APPENDIX 3.1: RATIONALE FOR USE OF FOCUS GROUPS}

Focus groups are a valuable qualitative method for discovery and exploration when little is known about a particular topic (Hawthorne et al., 2006). Commonly used in social science research, focus groups are defined as "a carefully planned discussion designed to obtain perceptions on a defined area of interest in a permissive, nonthreatening environment" (Kreuger, 1994, p.6). They are considered particularly suited to the study of attitudes and experiences (Kitzinger, 1995), and can be used alone or in combination with other, often more quantitative, research methods such as surveys, providing a means of data triangulation (or approaching the same phenomenon from different perspectives; Willig, 2001).

In practice, the focus group method is commonly used to supplement more traditional data-collection methods since it can provide an alternative perspective (Millward, 2006). For example, focus groups may be used to enhance existing questionnaires since they promote discussion, accessing and sharing attitudes, perceptions and opinions through dynamic group interaction, and have the potential to elicit a rich array of positive and negative views resulting in measure refinement and improved validity (Kitzinger, 1995).

Focus groups may also be conducive to the discussion of 'sensitive' research questions. While some researchers have argued that semi-structured interviews may be more suited to this purpose (e.g. Willig, 2001), others point out that group work can actively facilitate personal disclosure and that focus groups are an ideal forum within which to empower vulnerable client groups to express their opinions and 
views (Wilkinson, 2003). Current debates centre on whether disclosure is facilitated more through homogeneous groups that have something in common (e.g. a shared history or experience) or through groups comprised of divergent individuals with no prior knowledge of one another. In practice it is recommended that group members share at least some common characteristics to facilitate disclosure since this aids the development of group rapport (Millward, 2006). While it is not the intention of focus group methodology to yield generalisable data, it is important that the focus group sample be chosen on theoretical grounds to reflect the broader population of interest and to provide meaningful information on the topic in question. 


\section{APPENDIX 3.2: Participant Information Sheet - Focus Group (Non-Clinical Sample)}

\section{Older Peoples' Attitudes to Mental III-health Information for Prospective Participants}

Thank you for reading this.

\section{$\bullet$ Introduction •}

You are being invited to take part in a research study about the views of older adults toward mental ill-health. Before you decide if you would like to participate it is important that you know a bit more about the study and what participation will involve. Please take time to read the following information carefully and raise any questions you may have with the researcher. Please ask if there is anything you are not clear about or if you would like more information.

Your views are very important to us. By taking part you will provide valuable information about the views and needs of older adults. This will help us to tackle stigma and discrimination in relation to older adults with mental health difficulties. Your contribution will be used to help the researchers to target important areas of inquiry and to formulate specific questions about attitudes, behaviours, and general well-being.

\section{$\bullet$ What are we asking of you?}

We would like to ask you to take part in this study by agreeing to join in a single focus group discussion together with peers from the meeting you currently attend through , . The focus group will take about $1 \frac{1}{2}-2$ hours (with a break) and will take the form of a general discussion about attitudes to mental ill-health in older adults. As part of the discussion, we will ask you to give your opinion about an attitudinal questionnaire and to consider the relevance of developing some additional questions. In the group there will be six to eight older adults and two facilitators. With your permission, we would also like to tape the discussion. This is purely to help us with analysing the information discussed on the day. It will be used by the research team only and you will not be identified in the results.

Your participation in the study is of course entirely voluntary. You can choose not to take part, or to withdraw from the study at any time without giving reasons. If you do choose to take part, any data you do give will be 
kept absolutely confidential. It will be viewed by the study researchers only. The tape will be kept securely and then erased after it has been transcribed.

\section{-What to do now?}

If you do not wish to take part, you need do nothing more. Thank you for taking the time to read about our study.

\section{If you decide to participate:}

We are delighted that you have chosen to take part. We would like to thank you in advance for your co-operation. A member of the staff will make arrangements for you to attend the focus group, which will take place on [date and time]. You will receive details of this shortly. Transport to the venue will be arranged on your behalf and we will also cover any additional costs incurred by you due to participation. On the day you will be asked to complete a consent form giving your permission to participate.

We enclose a copy of the study questionnaire and covering letter/information sheet for your interest and would be grateful if you could look over these prior to the group. You do not need to complete the questionnaire but you may do so if you wish. Your data will not be used in the final study.

If you have any questions about the project please ask the study researcher directly, or contact a member of staff from We would be very pleased to answer any queries you may have.

\section{Study Researcher}

Department of Clinical Psychology

Tel No:

email: 


\section{APPENDIX 3.3: CONSENT FORM (FOCUS GROUP)}

\section{Informed Consent Form}

\section{Older peoples' attitudes to mental ill-health: Focus Group \\ Principal Researcher:}

Please tick box

- I confirm that I have read and understand the information sheet dated 23 August, 2006 (Version: 6) for the above study. I have had the opportunity to consider the information, ask questions, and have had these answered satisfactorily.

- I understand that my participation is voluntary and that I am free to withdraw at any time, without giving any reason, without my medical care or legal rights being affected.

- I understand that the information I provide during this study may be looked at by responsible individuals from [...]/[...], where it is relevant to my taking part in this research. I give permission for these individuals to have access to my information.

- I am aware that all information collected during this study will be recorded on tape and transcribed in its entirety. I understand that I will not be identified in the transcript or in any results emanating from this research.

- I understand that the focus group tape will be destroyed once the transcript is complete.
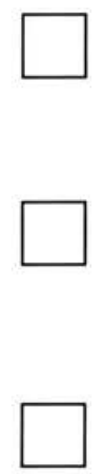

- I agree to take part in the above study.

Name of participant

Name of person taking consent (if different from researcher)

Name of researcher

\section{Date}

Date

Date
Signature

Signature

Signature 


\section{APPENDIX 3.4: FOCUS GROUP GUIDELINES}

\section{General Aims}

\section{Overall Aim of Focus Groups:}

To undertake semi-structured focus groups with older adult participants, in order to explore attitudes to mental ill-health.

\section{Potential topic areas:}

- Exploration of terminology (e.g. mental illness; mental health difficulties).

- To ascertain the ways in which mental health difficulties might impact on an individual and his/her family.

- Exploration of societal and personal views of mental illness a) in general, and $b$ ) in relation to older people.

- Discussion of modes of support/help/awareness of resources.

- Discussion of potential treatment seeking barriers (including shame and discrimination)?

\section{Methodology}

\section{Number of Groups and Composition}

To undertake two focus groups (one with a clinical sample; one with a community sample) to address key issues in open-group discussion format. Groups should comprise a minimum of six participants.

Group A: will comprise participants from the [...] [...] User Panels, preferably of mixed gender, age range (65 upwards), with mixed health status.

Group B: will comprise patients attending [...] Day Hospital, be of mixed gender, age range (65 upwards), with mixed health status.

There is no requirement for members of the group to be familiar with one other.

\section{Timing of groups}

To some extent this will depend on group composition and dynamics. Each group should last no longer than 1.5-2 hours, and should be punctuated with at least one coffee break.

\section{Materials}

Participants will be provided with copies of the questionnaire, information sheets, and study information letter prior to the day of the focus group and invited to review these in advance of the group. Additional copies of the questionnaire, displaying different design formats, will be provided at the focus group. 


\section{Proposed Content and Procedure for Running Groups}

\section{Content}

The following list represents the "core" information to be collected during focus group sessions.

$>$ Free-form discussion of attitudes to mental ill-health.

$>$ Structured discussion of questionnaire and study materials.

$>$ Suggestions for revisions.

\section{All groups:}

$>$ Consent forms

$>$ Collation of socio-demographic data

$>$ Moderator documentation of focus group sessions

Socio-demographic information and informed consent forms should be collected at the beginning of the session. In addition, moderators should collect information documenting the group session, including: location, venue, group composition, duration, day/date/time, moderators involved, general atmosphere of group, nature of discussion flow, and problems encountered.

\section{PROCEDURE I}

\section{Orientation}

\section{$10 \mathrm{mins}$}

\section{Welcome and Introductions \\ Initial orientation to focus group}

The Scottish Executive is currently undertaking some research to find out about societal attitudes to mental illness/mental ill-health but to date this has been directed largely towards the younger adult population. As part of a joint NHS/University research study I am interested in finding out about older people's views on mental illness and would like to ask you to take part in a focus group to explore this area further. The focus group will be structured into two parts. The first part will involve a general group discussion around the topic of attitudes to mental health problems - the idea of this part is to explore your own personal views and attitudes toward mental health and your views of how you think society views and treats people (particularly older people) with mental health difficulties; in the second part of the focus group we would like to ask you for some feedback about the Attitudes to Mental III-Health Questionnaire. This is so we can improve the questionnaire prior to sending it out to study participants.

If you are happy to take part in today's focus group, please read the focus group information sheet and sign and date the informed consent form. We would also be grateful if you could take a couple of minutes to complete the sociodemographics sheet, which provides us with a little bit more information about you. Please note however that any information you provide in this focus group is entirely confidential.

Are there any questions before we begin? 
In accordance with criteria for focus group planning outlined by Kreuger (1994), the discussion will be guided using a number of different categories of open-ended questions, as outlined below:

Introductory Question

Aim: To begin discussion of the topic.

Q: What does the term 'mental illness/mental ill-health' mean to you?

or

Q: When you think of the term 'mental illness' what comes to mind?

Following a general opening discussion, a small number of transition and key questions can be asked to encourage further discussion, such as:

\section{Transition Question}

Aim: To move the conversation toward the key questions that drive the study.

Q: In what ways might mental health difficulties affect an individual and his/her family? (Prompts: self-views; societal views; engagement; treatment health, government agencies, banks, clubs, other people)

Key Questions

Aim: Obtain insight on areas of central concern in the study.

Q: How do you think society views people with mental health difficulties?

Q: Do you think attitudes vary according to age?

$Q:$ How do you think society views older people in general?

Q: What are your own views about younger (and older) people with mental health difficulties?

\section{Prompts:}

a. Examples of negative views?

b. Examples of positive views?

c. Where do you think these views come from?

d. What are the specific issues in relation to older people?

e. Experiences of shame or discrimination?

f. Impact on treatment-seeking and delivery?

g. Other obstacles to seeking or receiving treatment (eg transport,

h. Additional areas?

Ending Question(s)

Aim: Helps researchers determine where to place emphasis and brings closure to the discussion.

Q. Is there anything else we have not discussed that you think might be relevant to this research?

or

Q. Is there anything else you would like to add? 


\section{1/10/2006}

Participants: 6 (female; age range 79-86)

Moderators: 2

\section{PART A: Open Discussion}

1 Moderator 1: Thank you very much for taking the time to read all that information and agreeing to take part. As I said at the beginning, what I thought would be useful to do, certainly from our point of view, would be just to start a general discussion for the next 25 or 30 minutes or so about your views about mental illness and mental ill health. I am using these terms 'mental illness' and 'mental ill health' because these are the terms that we are familiar with, but I don't want to assume that those are the terms that you would necessarily use or would know, so if there is another term that you think you would use or hear about more frequently, then please feel free to let us know what that is, but I thought if we could kick off and just ask you, what are your views about mental ill health, mental illness in the general population.

13 Participant 1: $\quad$ Some people have no contact with anybody like that so they don't know what it is really. I have, but a lot of people don't know what mental illness is, or understand it.

16 Participant 2: $\quad$ its an illness just like any other illness, as ... says a lot of folk don't really understand that

18 Participant 3: a lot of people think of mental illness as really needing to be in an institution..... (general agreement that 'that's not right') and I think if they gave it another name, people say, oh its their nerves, that sounds better to me than mentally ill, people suffering with their nerves or suffering mental illness, I know which one I would rather have

24 Moderator 1: that's a interesting point actually - you have said there that nerves is a more common expression perhaps

26 Participant 3: ... a kinder expression

27 Participant 1: I think the word 'mental' is a sort of stigma, you know mental ...

29 Moderator 1: does anyone else feel that about that word, that it has quite negative connotations, what springs to mind really when you hear that word

32 Participant 2: somebody out of their mind! 
2 Participant 1: I think about work! (that's where I worked)

3 Participant 2: what would you say was the difference between a form 6.4 of dementia and mental illness, because is it true, its a condition with brain damage or something like that

6 Participant 1: same thing in the end

7 Moderator 1: Do you find that you equate the two together, mental illness and dementia

9 Participant 2: well my husband had dementia and it was caused through an accident and he was brain damaged.

11 Participant 4: going on from there though, as I get older anyway, I cant speak for anybody else, there is a certain fear you might lose some of your mental faculties and that bothers me, the fact that I might not be able to understand what people are saying, apart from deafness, and the fact that I cant understand things, these kind of things - it hasn't affected me yet! I think about it sometimes when I hear or see someone who is unfortunately not able to communicate properly, I think, you know, this could be me in a years time or even less because a friend of mine she was fine, she was quite compos mentis and then within 6 months she went, it was very very speedy - when we looked back we found little bits that we should have picked up on but we didn't and within 6 months she just was completely away, so obviously as I get older myself I think am I going to keep...

24 Participant 2: I don't think you realise though, you have got it, because I know what my husband was like, he thought the same thoughts normally but he wasn't and he didna realise it.

25 Participant 5: but is that not dementia, is dementia the same as mental illness

27 Moderator 1: well that's a good question isn't it and I suppose it depends on what we think mental illness actually is

29 Participant 5: this business of you forget things and you cant think of a word, I mean we all have that (general agreement that we all do have this) and it seems you get it more and more as you get older

32 Participant 4: I mean even when you are talking with somebody, you know that I do it - you sort of have a mental block, especially if you are going into a room for something, you forget what you have gone for - it is another mental block! 


\section{APPENDIX 3.5b - Focus Group 2 (Clinical) Transcript Excerpt}

9/11/2006

Index Ref:

Participants: 6 (4 females, 2 males; age range 71-80)

Moderators: 2

\section{PART A: Open Discussion}

1 Moderator 1: What I would like to do is maybe have 15 minutes or so just having a wee chat with you about your attitudes to mental illness. I know it can sometimes be a difficult subject to think about and it is not something we tend to talk about very often, is it? We talk more about our physical health than our mental health, but what I am interested in is just hearing what your views are about it. Does anybody have any views when you think about mental illness or mental ill-health - what tends to spring to mind for you?

8 Participant 1: Well I'll ask you something then,

9 Moderator 1: Yes

10 Participant 1: What made me take an overdose? There you go then! I started off in ..., about 40,50 years ago maybe 60 years ago, through what you had ..

13 Participant 2: Post natal depression

14 Participant 1: Twice with two babies.

15 Moderator 2: Would you associate something like that with mental health

16 Participant 1: I think that's what caused it. I used to - if anyone chapped on my door I locked myself in the toilet for no reason. I couldn't figure out a reason

17 Moderator 2: Yes

18 Participant 1: I just didn't understand it - they nurses could tell and I asked them how do you know when you're low? How do you know when, I'm a manic depressive and how do you know when your depressed?

21 Moderator 1: That's the difficult thing isn't it, cos sometimes other people can see it in you but it is hard for you to understand yourself - does anyone else have any similar experiences or thoughts on that? 
1 Moderator 2: Do you think when you hear the words for the mental health problems and the difficulties of seeing something that's frightening or something that you, what sort of emotions does it bring up or feelings.

4 Participant 1: I'll tell you something ..., are you happy to be talking to somebody, maybe just down in the bus stop and you just say "oh, I go to ... twice a week" and they say "oh, you're a nutter" ...

7 Moderator 2: right

8 Participant 1: So you ken to say you've been in ... and you dinnae ken any psychiatric patients.

10 Moderator 1: So what you're describing is there is a real stigma attached to it, yeah. Do other people agree with that? What do other people think?

12 Participant 3: Ah well a lot of people think that like you know and they always come here to think that they're going to improve, but eh, in general understand there's a lot of people came here and actually got worse than when they first came, so I don't know whether that's the tablets or whether it's the problem of coming here.

17 Moderator 1: Do people still get a sense of it being something that people are quite fearful of and quite discriminating of?

19 Participant 1: I tell them when I talk to them. I'm on $400 \mathrm{mg}$ of Lithium and it's a brain pill and Dr ... says just two or three weeks ago it's dangerous we'll put you down to two. Now if I had known it was a brain pill then I wouldn't have taken it. And yet I've been in ...had the head treatment - had everything. Now I'm fed up saying to ... that one of these mornings I'm gonna wake up and have nothing wrong with me. Ken what I mean.

25 Moderator 1: $\quad$ Yeah it feels that you've lived with that for a long time.

26 Participant 1: Yeah, I'm not getting out of it. Mentally I'm alright now.

27 Moderator 1: Do you think people in general are quite, how would you describe that - do you think people in general are quite disrespectful of people who have got mental health difficulties?

30 Participant 1: Yes I think so. Yes, yes, I think that people won't want to know me at all. They've never done ... and everything and I've been out it for years 


\section{APPENDIX 3.6: FOCUS GROUP PARTICIPANT CHARACTERISTICS}

\section{NON-CLINICAL GROUP}

In terms of non-clinical participant characteristics: all participants were living in their own home; five participants were widowed, one was separated/divorced; five out of six participants left school between the ages of 14-16, although one participant subsequently attained a college diploma; four participants had held administrative/secretarial occupations, two participants had prior training and employment experience in mental health nursing. In terms of experience of mental illness, one individual had past experience of mental health difficulties as a young adult for which she had received in-patient treatment, and one other participant had indirect experience via caring for her husband who had dementia; all rated their health as 'fairly good' despite most participants suffering a range of chronic health conditions including asthma, diabetes, and arthritis; all participants were taking some form of medication.

\section{CLINICAL GROUP}

In terms of clinical participant characteristics: all participants were living at home with the support of family/carers; three participants were separated/divorced, two were married, one was single; all participants had left school between the ages of 1416; both male participants had held skilled manual occupations (e.g. joiner), while female participants had held a range of jobs from factory work to nursing; five participants described their health as 'good' or 'fairly good', one participant described it as 'not good'; overall, few medical conditions were noted, and only three participants were taking regular medication (mainly psychotropics). 


\section{APPENDIX 3.7: PROPOSED QUESTIONNAIRE MODIFICATIONS}

\begin{tabular}{|l|l|l|}
\hline Section & Focus Group 1 (Non-clinical) & Focus Group 2 (Clinical) \\
\hline Front Cover & Preferred flower design. & Preferred flower design. \\
\hline $\begin{array}{l}\text { Part 1: } \\
\text { Sociodemographics }\end{array}$ & $\begin{array}{l}\text { Q6 Change wording of item: } \\
\text { 'Living at home }\end{array}$ & $\begin{array}{l}\text { Q4 Change wording of item: } \\
\text { 'High school' to 'Secondary } \\
\text { school' }\end{array}$ \\
to & $\begin{array}{l}\text { Q9 Add examples } \\
\text { 'Yes, in the past I have } \\
\text { previously attended mental } \\
\text { health services (e.g. } \\
\text { psychology, psychiatry, day } \\
\text { hospital etc.)' }\end{array}$ \\
\hline $\begin{array}{l}\text { Part 2: Attitudes to } \\
\text { Mental Ill-Health }\end{array}$ & $\begin{array}{l}\text { Q6 Change wording of item*: } \\
\text { 'I would see a psychologist } \\
\text { because normal people go to } \\
\text { psychotherapy' }\end{array}$ & $\begin{array}{l}\text { None. } \\
\text { to }\end{array}$ \\
$\begin{array}{l}\text { Part 5: Realth Behaviours } \\
\text { Checklist }\end{array}$ & $\begin{array}{l}\text { Add response option headers } \\
\text { (i.e, 'Never' - 'Always') to top } \\
\text { of page 18. }\end{array}$ & $\begin{array}{l}\text { None. } \\
\text { because its normal for people }\end{array}$ \\
\hline
\end{tabular}

* Suggested change not implemented as pre-existing measure with established psychometric properties. 


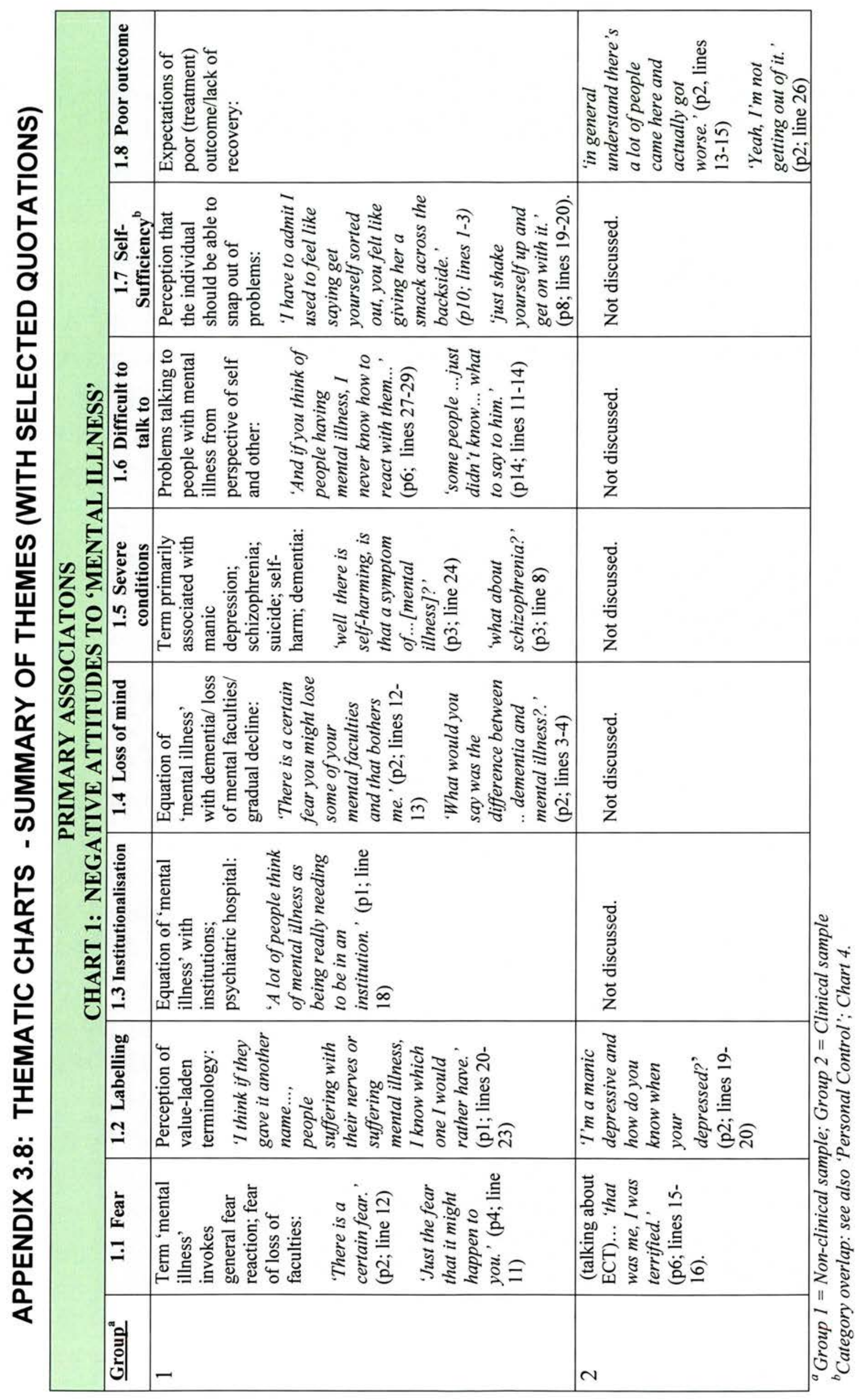




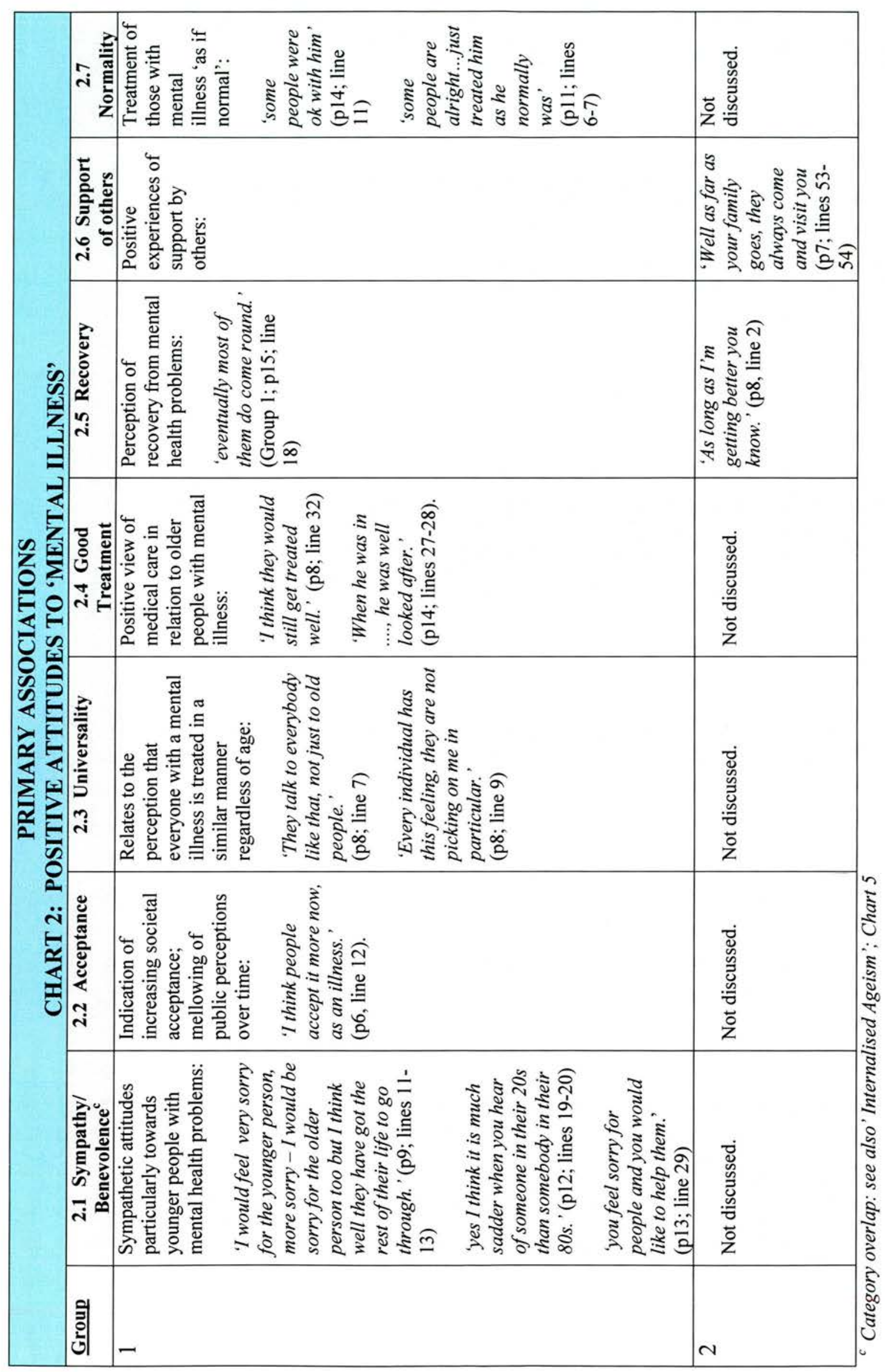




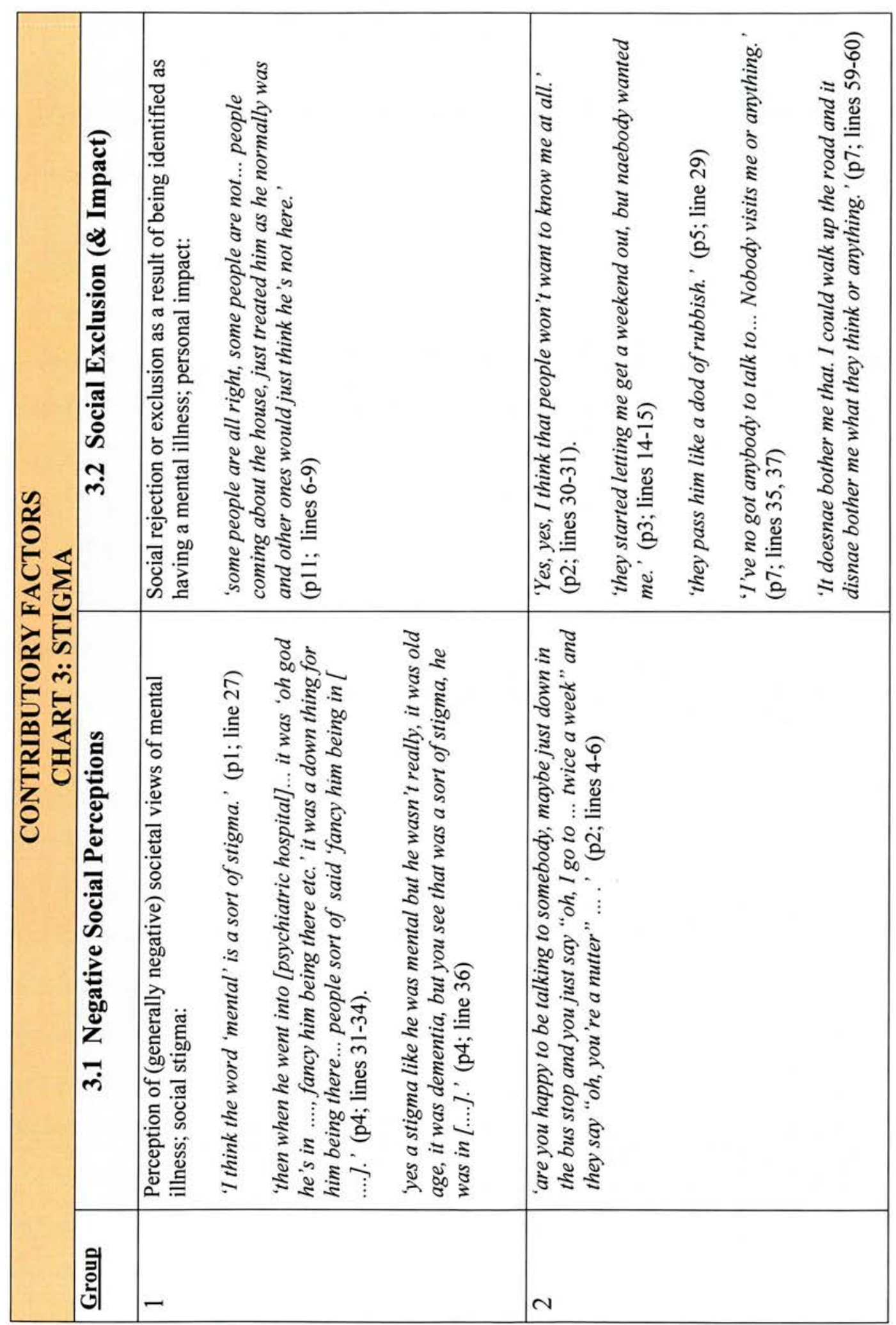




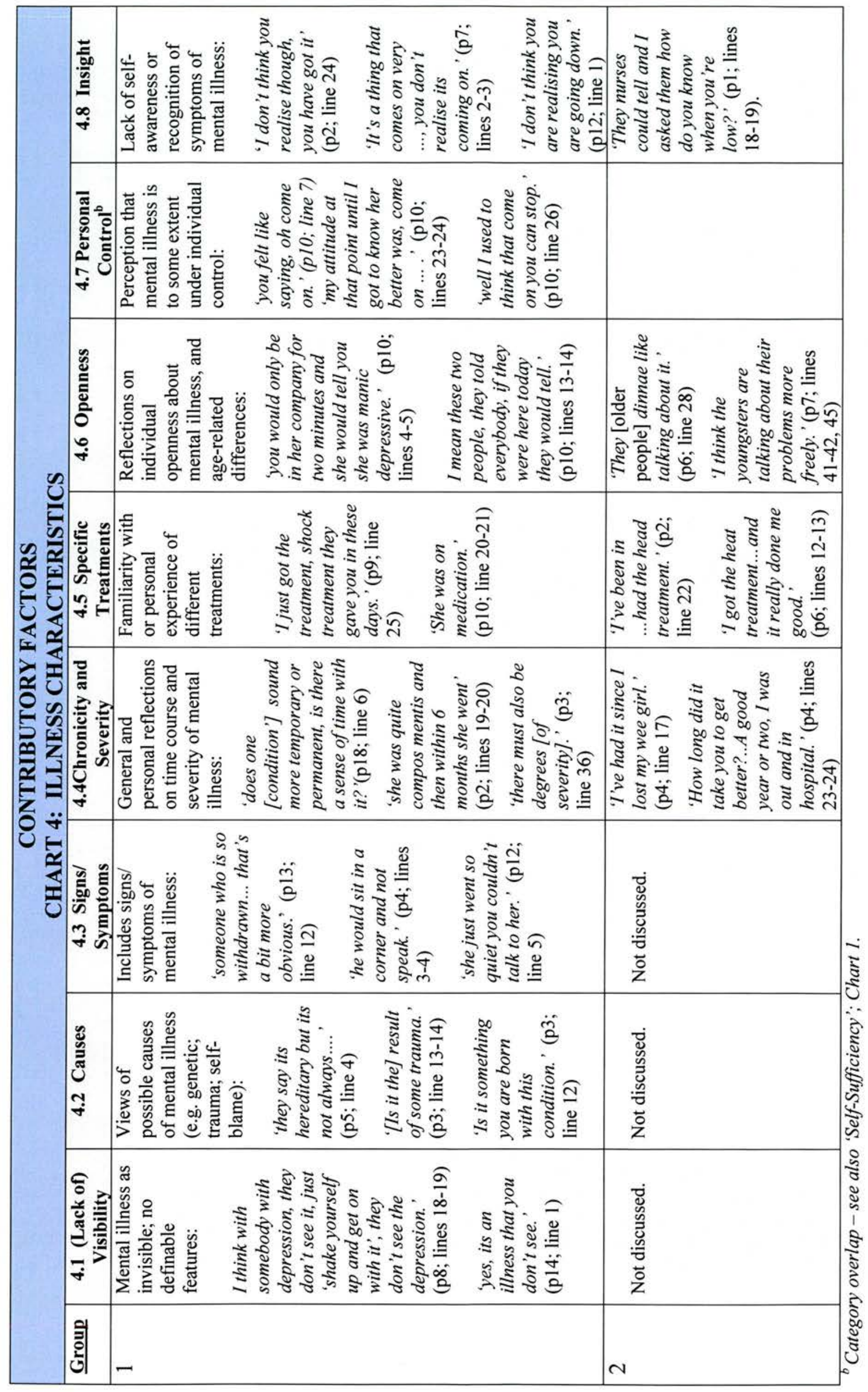




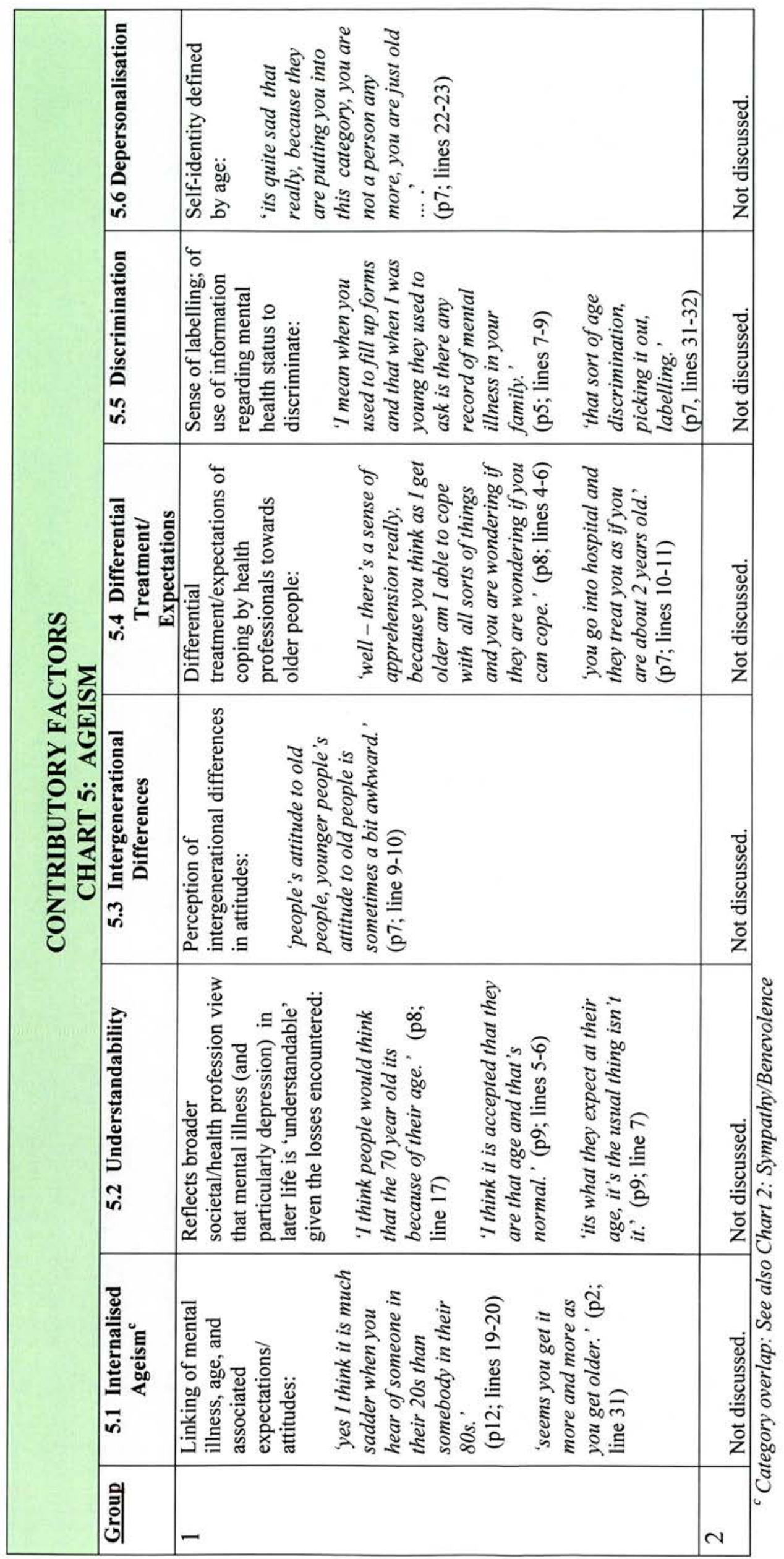




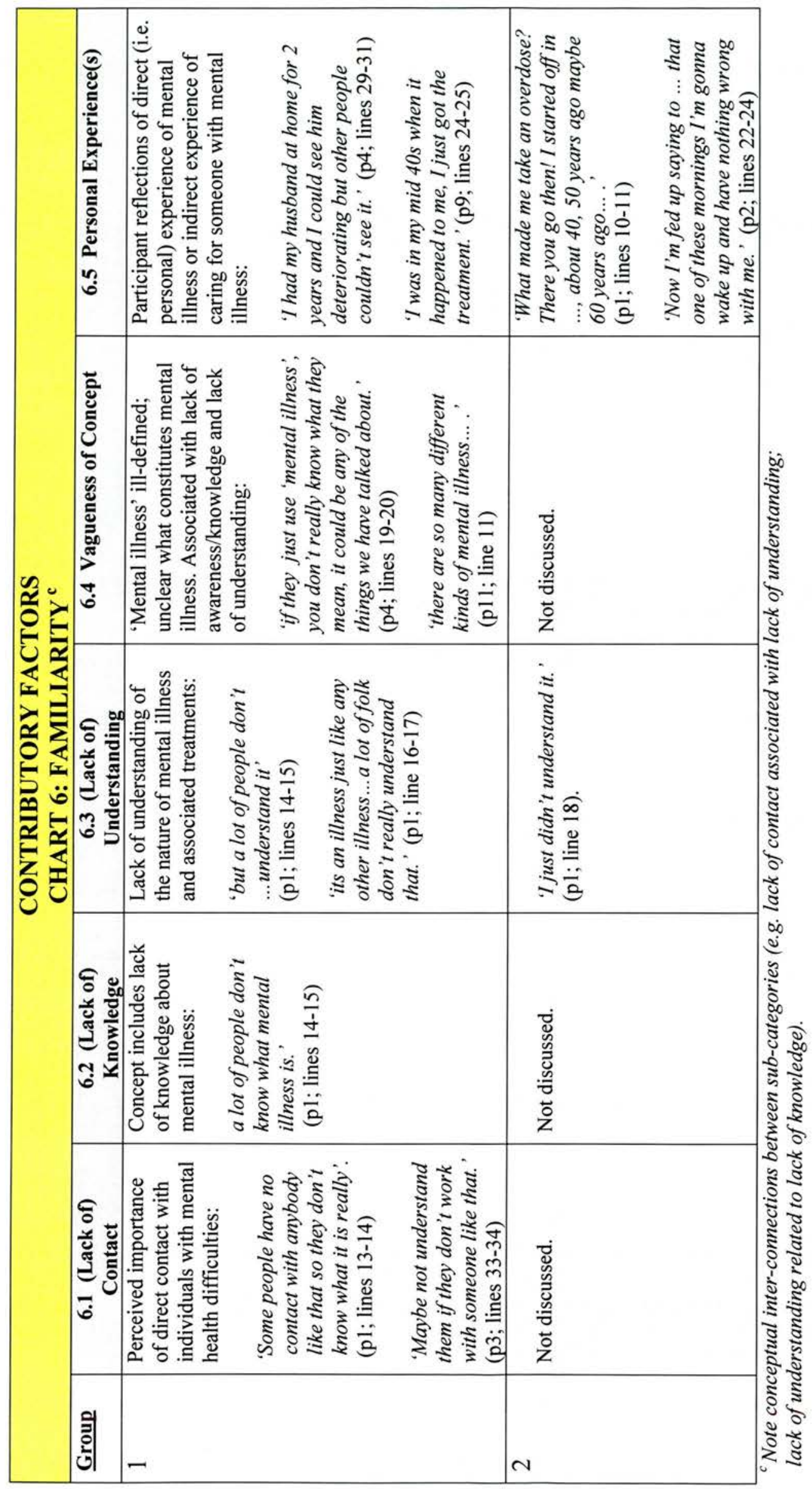




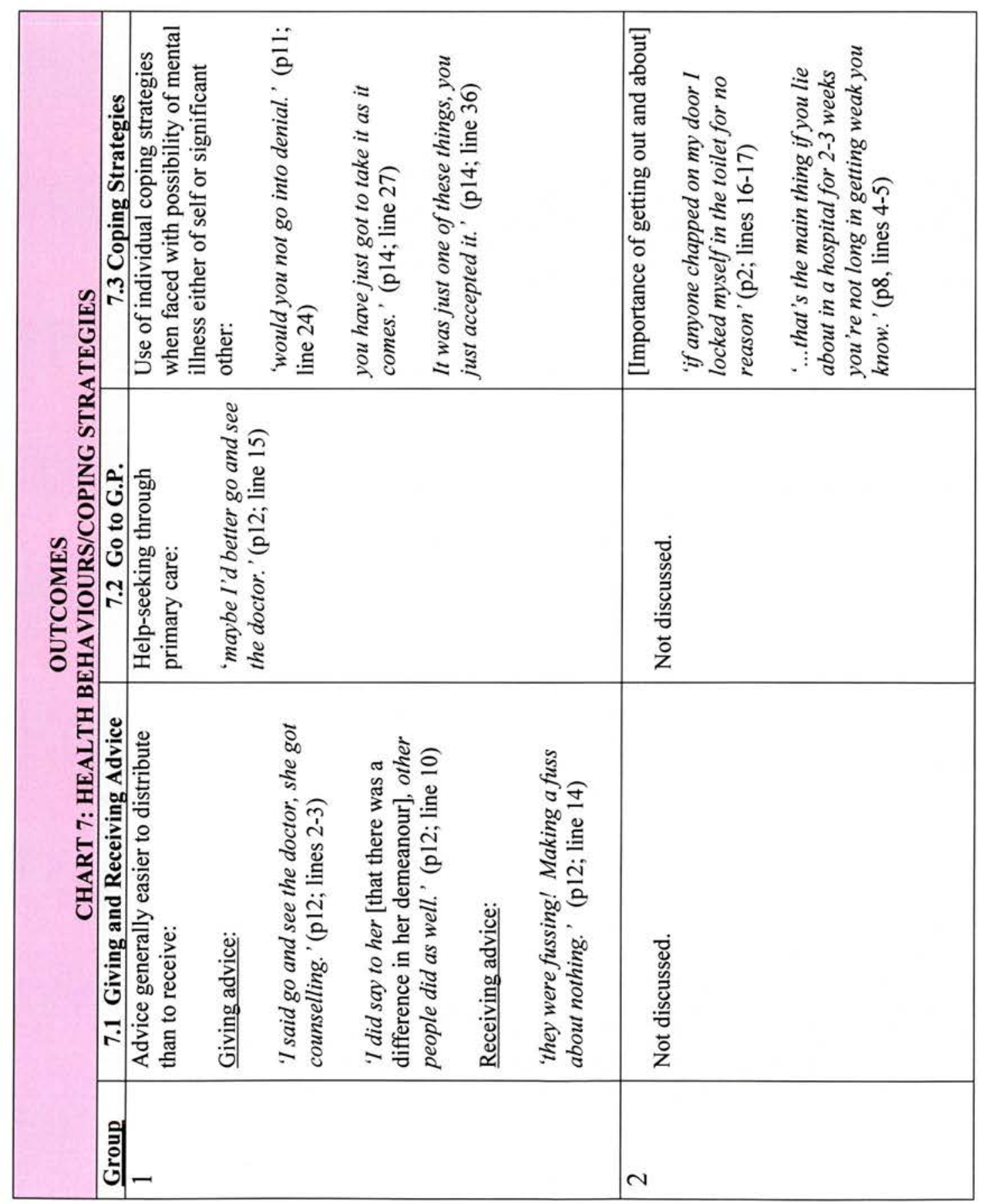




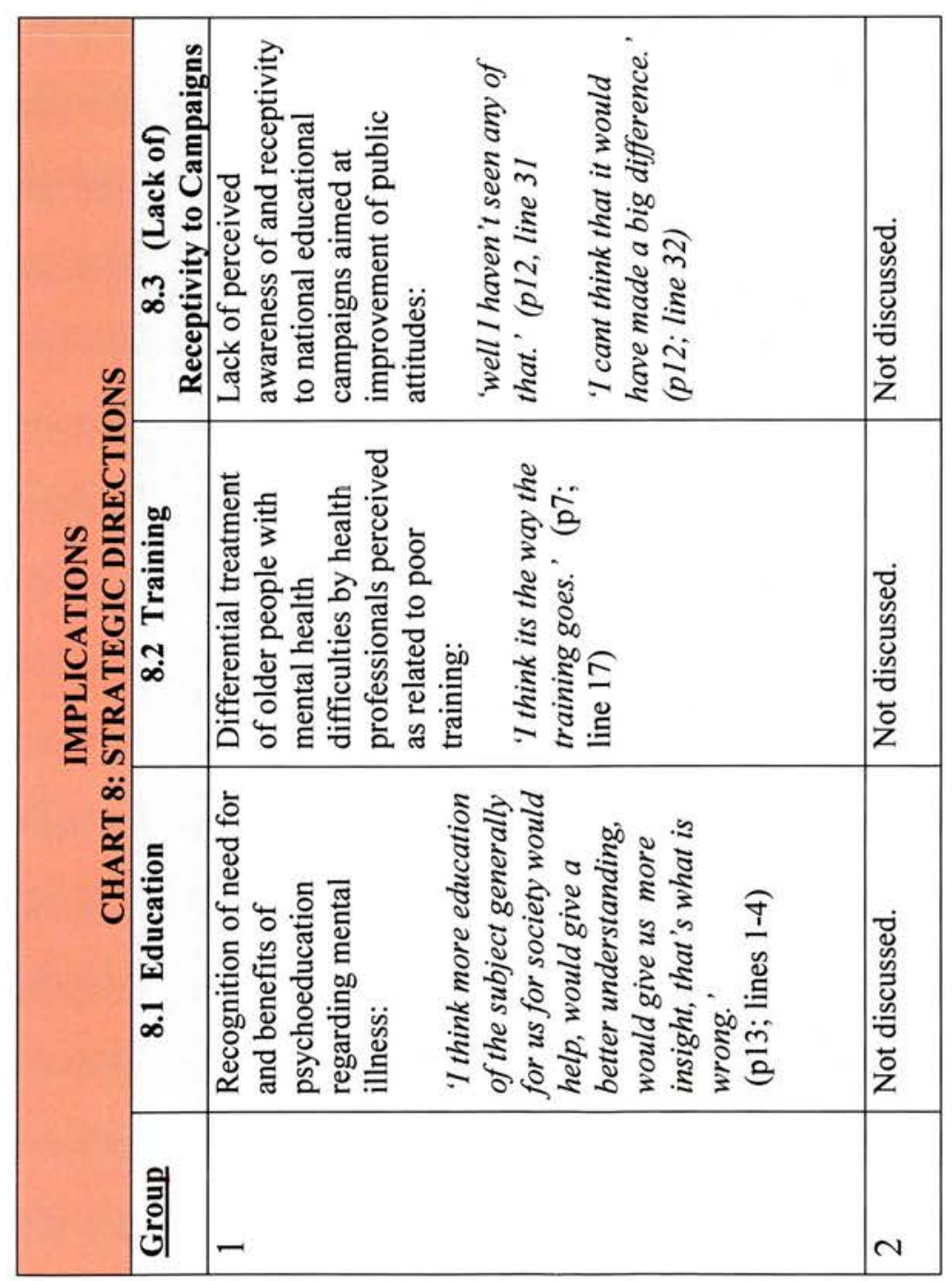




\section{APPENDIX 3.9: QUALITY CONTROL AND ETHICAL ISSUES IN QUALITATIVE ANALYSIS}

\section{Quality Control}

In accordance with qualitative principles, issues of reliability and validity were addressed in the current study in terms of 'transparency', 'rigour', and 'coherence' (Yardley, 2000). Transparency was achieved through detailed description of methodology (see Section 3.1). Cross-validation of outcomes with existing literature, and data triangulation (i.e. with quantitative data analysis outcomes) were considered important methods of improving rigour (Lacey \& Luff, 2001). In addition, emergent themes were independently reviewed by a second researcher to enhance coherence (i.e. to determine whether the framework being constructed was a convincing account of the data). Discrepancies in coding were discussed and a consensus reached regarding appropriate modifications to the thematic framework. This primarily involved the inclusion of two additional thematic sub-categories to improve clarity (see categories 2.7 and 4.8 in Table 5).

\section{Ethical Issues Pertaining to Focus Groups}

Possible ethical issues associated with focus group participation, such as participant disclosure of personal experiences of mental illness, were discussed in advance of the groups. Confidentiality was assured by requesting participants to refrain from discussing information raised during the group outwith the focus group session, and through subsequent anonymisation of focus group transcripts. Appropriate procedures were adhered to concerning informed consent. Following the focus group interviews, participants were thanked for their involvement, and provided with the study researcher's contact details in case they wanted to get in touch regarding any issues that had arisen during the group session.

Peer supervision was undertaken between moderators following each focus group interview to allow an opportunity for debriefing, self-reflection, and to discuss any process and content issues as appropriate. 


\section{APPENDIX 3.10: KEY THEMES OF MENTAL ILLNESS}

\begin{tabular}{|c|c|c|c|}
\hline $\begin{array}{c}\text { Broad } \\
\text { Category }\end{array}$ & $\begin{array}{l}\text { Major } \\
\text { Theme }\end{array}$ & $\begin{array}{l}\text { Constituent Themes - } \\
\text { Group } 1 \text { (Non-clinical) }^{\text {a }}\end{array}$ & $\begin{array}{l}\text { Constituent Themes - } \\
\text { Group } 2 \text { (Clinical) }{ }^{\text {b }}\end{array}$ \\
\hline $\begin{array}{l}\text { Primary } \\
\text { Associations }\end{array}$ & $\begin{array}{l}\text { Negative } \\
\text { Attitudes }\end{array}$ & $\begin{array}{l}\text { Fear [1.1] } \\
\text { Labelling [1.2] } \\
\text { Institutionalisation/Hospitalisation [1.3] } \\
\text { Loss of mind [1.4] } \\
\text { Severe conditions [1.5] } \\
\text { Difficult to talk to [1.6] } \\
\text { Self-sufficiency }{ }^{c}[1.7] \\
\text { Individual reactions [1.8] }\end{array}$ & $\begin{array}{l}\text { Fear [1.1] } \\
\text { Labelling [1.2] } \\
\text { Poor outcome [1.9] }\end{array}$ \\
\hline $\begin{array}{l}\text { Primary } \\
\text { Associations }\end{array}$ & $\begin{array}{l}\text { Positive } \\
\text { Attitudes }\end{array}$ & $\begin{array}{l}\text { Sympathy/benevolence }^{\mathrm{d}}[2.1] \\
\text { Societal acceptance }[2.2] \\
\text { Universality [2.3] } \\
\text { Good treatment [2.4] } \\
\text { Recovery [2.5] } \\
\text { Normality [2.7] }\end{array}$ & $\begin{array}{l}\text { Recovery [2.5] } \\
\text { Support of others [2.6] }\end{array}$ \\
\hline $\begin{array}{l}\text { Contributory } \\
\text { Factors }\end{array}$ & Stigma & $\begin{array}{l}\text { Negative societal perceptions [3.1] } \\
\text { Social exclusion (\& impact) [3.2] }\end{array}$ & $\begin{array}{l}\text { Negative societal perceptions [3.1] } \\
\text { Social exclusion (\& impact) [3.2] }\end{array}$ \\
\hline $\begin{array}{l}\text { Contributory } \\
\text { Factors }\end{array}$ & $\begin{array}{l}\text { Illness } \\
\text { Characteristics }\end{array}$ & $\begin{array}{l}\text { (Lack of) Visibility [4.1] } \\
\text { Causes [4.2] } \\
\text { Symptoms [4.3] } \\
\text { Chronicity and severity }[4.4] \\
\text { Specific treatments [4.5] } \\
\text { Openness [4.6] } \\
\text { Personal Control }{ }^{\mathrm{c}}[4.7] \\
\text { Insight [4.8] }^{\text {[4 }}\end{array}$ & $\begin{array}{l}\text { Chronicity and severity }[4.4] \\
\text { Specific treatments }[4.5] \\
\text { Openness [4.6] } \\
\text { Insight }[4.8]\end{array}$ \\
\hline $\begin{array}{l}\text { Contributory } \\
\text { Factors }\end{array}$ & Ageism & $\begin{array}{l}\text { Internalised ageism }^{\mathrm{d}}[5.1] \\
\text { Understandability [5.2] } \\
\text { Intergenerational differences [5.3] } \\
\text { Differential treatment/expectations [5.4] } \\
\text { Discrimination [5.5] } \\
\text { Depersonalisation [5.6] }\end{array}$ & \\
\hline $\begin{array}{l}\text { Contributory } \\
\text { Factors }\end{array}$ & Familiarity $^{\mathrm{e}}$ & $\begin{array}{l}\text { (Lack of) Contact [6.1] } \\
\text { (Lack of) knowledge [6.2] } \\
\text { (Lack of) Understanding [6.3] } \\
\text { Vagueness of concept [6.4] } \\
\text { Personal experience [6.5] }\end{array}$ & $\begin{array}{l}\text { (Lack of) Understanding [6.3] } \\
\text { Personal experience [6.5] }\end{array}$ \\
\hline Outcomes & $\begin{array}{l}\text { Health } \\
\text { Behaviours/ } \\
\text { Coping } \\
\text { Strategies } \\
\end{array}$ & $\begin{array}{l}\text { Giving and receiving advice [7.1] } \\
\text { Go to G.P. [7.2] } \\
\text { Coping strategies [7.3] }\end{array}$ & Coping strategies [7.3] \\
\hline Implications & $\begin{array}{l}\text { Strategic } \\
\text { Directions }\end{array}$ & $\begin{array}{l}\text { Education [8.1] } \\
\text { Training [8.2] } \\
\text { Receptivity to educational campaigns [8.3] }\end{array}$ & \\
\hline
\end{tabular}

${ }^{a}$ Numbers in square parentheses relate to thematic framework index (Table 5). Thematic charts (Appendix

3.8) provide details of group source of theme, description of constituent themes and selected quotations.

${ }^{b}$ Themes outlined in blue font indicate common themes identified across both groups.

${ }^{c}$ Category overlap: Self-sufficiency [1.7] and Personal Control [4.7].

${ }^{d}$ Category overlap: Sympathy/Benevolence [2.1] and Internalised Ageism [5.1]

${ }^{e}$ Note conceptual inter-connections between sub-categories of Familiarity theme (e.g. lack of contact

associated with lack of understanding; lack of understanding related to lack of knowledge/awareness etc.). 


\section{APPENDIX 4.1: TABLE OF AGE DESCRIPTIVES INDICATING DEVIATIONS FROM NORMALITY}

\begin{tabular}{|c|c|c|c|c|c|c|c|c|c|c|}
\hline Variable & Group* & Mean & SD & $\begin{array}{l}\text { Skew } \\
\text { (std. } \\
\text { error) }\end{array}$ & $\begin{array}{c}\mathbf{Z} \\
\text { Score }^{+}\end{array}$ & $\begin{array}{c}\text { Kurtosis } \\
\text { (std. } \\
\text { error) }\end{array}$ & $\begin{array}{c}\mathbf{Z} \\
\text { Score }^{+}\end{array}$ & $\mathbf{K}-\mathbf{S}^{++}$ & $\begin{array}{c}\text { p } \\
\text { value }\end{array}$ & $\begin{array}{c}\text { Signif. } \\
\text { Deviation }\end{array}$ \\
\hline \multirow[t]{3}{*}{ Age } & All & 77.63 & $\begin{array}{c}8.0 \\
4 \\
\end{array}$ & $\begin{array}{c}0.47 \\
(0.28) \\
\end{array}$ & 1.68 & $\begin{array}{c}-0.43 \\
(0.56) \\
\end{array}$ & -0.77 & 0.11 & $\mathrm{p}=0.05$ & $x$ \\
\hline & 1 & 79.84 & $\begin{array}{c}8.0 \\
5\end{array}$ & $\begin{array}{c}0.23 \\
(0.34)\end{array}$ & 0.68 & $\begin{array}{c}-0.54 \\
(0.66)\end{array}$ & -0.82 & 0.11 & $\mathrm{p}=0.20$ & $x$ \\
\hline & 2 & 72.59 & $\begin{array}{c}5.4 \\
2\end{array}$ & $\begin{array}{c}0.56 \\
(0.49)\end{array}$ & 1.14 & $\begin{array}{l}-0.89 \\
(0.95)\end{array}$ & -0.94 & 0.18 & $\mathrm{p}=0.06$ & $x$ \\
\hline
\end{tabular}

*All $=$ total sample $(n=72) ; 1=$ non-clinical sample $(n=50) ; 2=$ clinical sample $(n=22)$

+ Absolute value greater than 3.29 is significant at $p<0.001$ level. . Significant values highlighted in bold.

$++K-S=$ Kolmogorov-Smirnov one-sample test which tests whether the distribution as a whole deviates from a normal distribution; $\mathrm{df}=72$ (whole sample); $\mathrm{df}=50$ (Group1); $\mathrm{df}=22$ (Group 2). Alpha set at $p<0.001$ for multiple tests of normality. 


\section{APPENDIX 4.2: HISTOGRAMS OF AGE DISTRIBUTION}

\section{AGE}

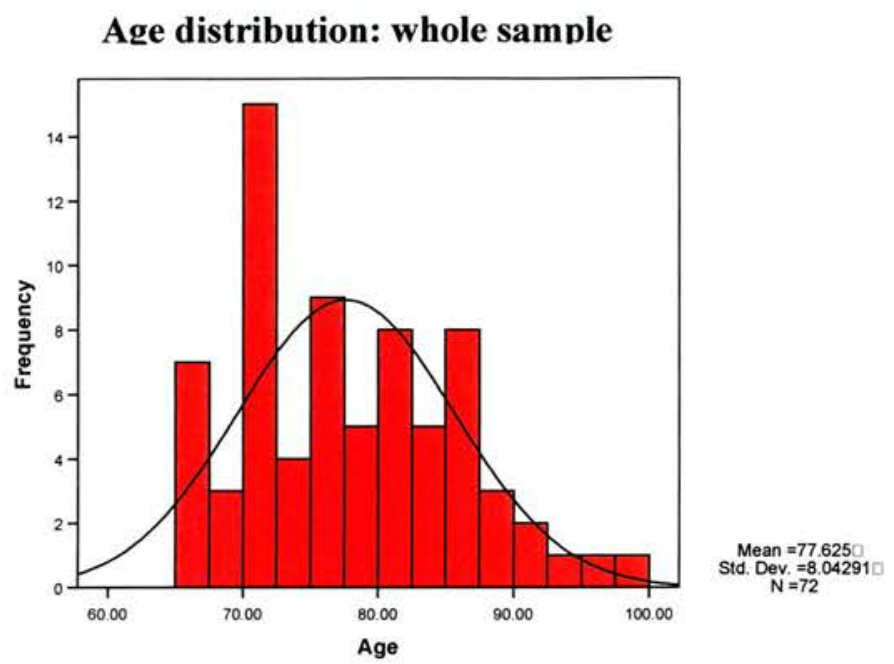

Age distribution: Non-clinical sample

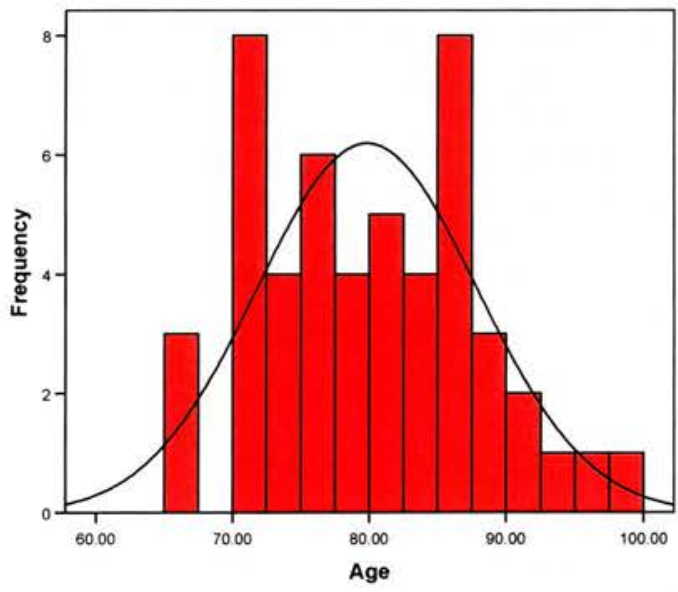

$\begin{aligned} \text { Mean } & =79.84 \\ \text { std. Dev. } & =8.0467\end{aligned}$

Age distribution: Clinical sample

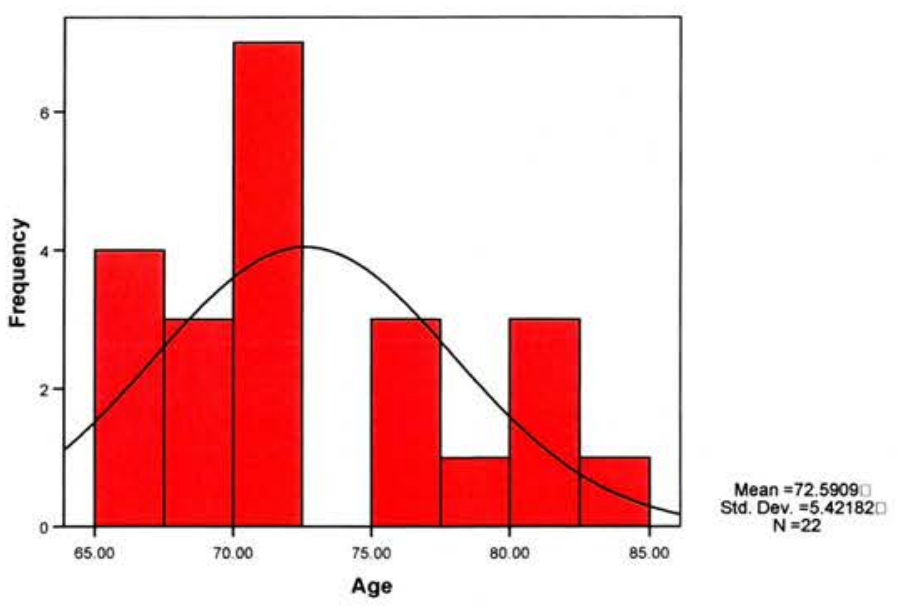


APPENDIX 4.3: BIVARIATE CORRELATIONS BETWEEN AGE AND STUDY VARIABLES.

\begin{tabular}{|l|l|c|c|c|}
\hline \multicolumn{2}{|c|}{ Variable } & $\boldsymbol{r}$ & Signif. (2-tailed) & N \\
\hline \multirow{3}{*}{ BMHSS } & Ageism & 0.27 & $\mathrm{p}=\mathbf{0 . 0 2}$ & 72 \\
\cline { 2 - 5 } & Stigma & 0.14 & $\mathrm{p}=0.25$ & 72 \\
\hline Understandability & 0.19 & $\mathrm{p}=0.11$ & 72 \\
\hline \multirow{4}{*}{ AAQ } & Composite & -0.19 & $\mathrm{p}=0.11$ & 72 \\
\cline { 2 - 5 } & Growth & 0.22 & $\mathrm{p}=0.06$ & 72 \\
\cline { 2 - 5 } & Loss & 0.07 & $\mathrm{p}=0.55$ & 72 \\
\cline { 2 - 5 } & Physical & $\mathbf{0 . 3 2}$ & $\mathrm{p}=\mathbf{0 . 0 0 7}$ & $\mathbf{7 2}$ \\
\hline \multirow{3}{*}{ Rame } & 0.06 & $\mathrm{p}=0.60$ & 72 \\
\hline \multirow{3}{*}{ GHQ-12 } & & & \\
\hline \multirow{3}{*}{ RHBC } & Composite & 0.19 & $\mathrm{p}=0.11$ & 72 \\
\cline { 3 - 6 } & Health-Action & 0.13 & $\mathrm{p}=0.14$ & 72 \\
\cline { 2 - 5 } & $\begin{array}{l}\text { Cognitive- } \\
\text { Affective }\end{array}$ & 0.17 & $\mathrm{p}=0.28$ & 72 \\
\hline
\end{tabular}


APPENDIX 4.4: TABLE OF SCALE DESCRIPTIVES INDICATING DEVIATIONS FROM NORMALITY

\begin{tabular}{|c|c|c|c|c|c|c|c|c|c|c|c|}
\hline \multicolumn{2}{|c|}{ Measure } & \multirow{2}{*}{$\begin{array}{l}\text { Group } \\
\text { All }\end{array}$} & \multirow{2}{*}{$\begin{array}{l}\text { Mean } \\
13.74\end{array}$} & \multirow{2}{*}{\begin{tabular}{|l} 
SD \\
3.87 \\
\end{tabular}} & \multirow{2}{*}{$\begin{array}{c}\begin{array}{c}\text { Skew } \\
\text { (std. }\end{array} \\
\text { error) }\end{array}$} & \multirow{2}{*}{$\begin{array}{c}\begin{array}{c}\mathbf{Z} \\
\text { Score }^{+}\end{array} \\
4.64\end{array}$} & \multirow{2}{*}{$\begin{array}{c}\begin{array}{c}\text { Kurt } \\
\text { (std. }\end{array} \\
\text { error) }\end{array}$} & \multirow{2}{*}{$\begin{array}{c}\mathbf{Z} \\
\text { Score }^{+} \\
5.80\end{array}$} & \multirow{2}{*}{$\begin{array}{c}\mathbf{K}-\mathbf{S}^{++} \\
0.16\end{array}$} & \multirow{2}{*}{$\begin{array}{c}\begin{array}{c}p \\
\text { value }\end{array} \\
p< \\
0.001\end{array}$} & \multirow{2}{*}{$\begin{array}{c}\begin{array}{l}\text { Signif. } \\
\text { deviat. }\end{array} \\
\checkmark\end{array}$} \\
\hline BMHSS & Ageism & & & & & & & & & & \\
\hline & & 1 & 14.45 & 3.87 & $\begin{array}{c}1.36 \\
(0.34) \\
\end{array}$ & 4.00 & $\begin{array}{c}3.38 \\
(0.66) \\
\end{array}$ & 5.12 & 0.17 & $\begin{array}{c}\mathrm{p}< \\
0.001\end{array}$ & $\checkmark$ \\
\hline & & 2 & 12.27 & 3.48 & $\begin{array}{c}1.51 \\
(0.47) \\
\end{array}$ & 3.21 & $\begin{array}{c}5.10 \\
(0.92) \\
\end{array}$ & 5.54 & 0.27 & $\begin{array}{c}p< \\
0.001\end{array}$ & $\checkmark$ \\
\hline & Stigma & All & 9.87 & 2.54 & $\begin{array}{c}0.28 \\
(0.28) \\
\end{array}$ & 1.00 & $\begin{array}{c}0.55 \\
(0.55) \\
\end{array}$ & 1.00 & 0.14 & $\begin{array}{c}p= \\
0.002\end{array}$ & $x$ \\
\hline & & 1 & 10.00 & 2.52 & $\begin{array}{c}0.50 \\
(0.34) \\
\end{array}$ & 1.47 & $\begin{array}{c}1.14 \\
(0.66) \\
\end{array}$ & 1.73 & 0.14 & $\begin{array}{c}p= \\
0.02\end{array}$ & $x$ \\
\hline & & 2 & 9.58 & 2.60 & $\begin{array}{c}-0.14 \\
(0.47) \\
\end{array}$ & -0.30 & $\begin{array}{c}-0.62 \\
(0.92) \\
\end{array}$ & -0.67 & 0.13 & $\begin{array}{c}p= \\
0.20\end{array}$ & $x$ \\
\hline \multirow{3}{*}{\multicolumn{2}{|c|}{ Understandability }} & All & 8.96 & 3.51 & $\begin{array}{c}0.51 \\
(0.28) \\
\end{array}$ & 1.82 & $\begin{array}{c}1.09 \\
(0.55) \\
\end{array}$ & 1.98 & 0.16 & $\begin{array}{c}\mathrm{p}< \\
0.001\end{array}$ & $\checkmark$ \\
\hline & & 1 & 9.14 & 3.08 & $\begin{array}{c}0.70 \\
(0.34) \\
\end{array}$ & 2.06 & $\begin{array}{c}1.43 \\
(0.66) \\
\end{array}$ & 2.17 & 0.16 & $\begin{array}{c}p= \\
0.003\end{array}$ & $x$ \\
\hline & & 2 & 8.60 & 4.33 & $\begin{array}{c}0.93 \\
(0.47)\end{array}$ & 1.98 & $\begin{array}{c}0.79 \\
(0.92)\end{array}$ & 0.86 & 0.17 & $\begin{array}{l}p= \\
0.07\end{array}$ & $x$ \\
\hline \multirow{3}{*}{ AAQ } & \multirow[t]{3}{*}{ Composite } & All & 60.68 & 11.13 & $\begin{array}{c}-0.02 \\
(0.28)\end{array}$ & -0.07 & $\begin{array}{c}-0.37 \\
(0.55)\end{array}$ & -0.67 & 0.07 & $\begin{array}{l}p= \\
0.20\end{array}$ & $x$ \\
\hline & & 1 & 57.44 & 10.08 & $\begin{array}{c}-0.12 \\
(0.34)\end{array}$ & -0.35 & $\begin{array}{c}-0.24 \\
(0.66)\end{array}$ & -0.36 & 0.07 & $\begin{array}{l}p= \\
0.20\end{array}$ & $x$ \\
\hline & & 2 & 67.42 & 10.30 & $\begin{array}{c}-0.13 \\
(0.47)\end{array}$ & -0.28 & $\begin{array}{c}-1.01 \\
(0.92)\end{array}$ & -1.10 & 0.11 & $\begin{array}{c}p= \\
0.20\end{array}$ & $x$ \\
\hline & \multirow{3}{*}{ Growth } & All & 28.21 & 4.51 & $\begin{array}{c}-0.25 \\
(0.28) \\
\end{array}$ & -0.89 & $\begin{array}{c}-0.14 \\
(0.55)\end{array}$ & -0.25 & 0.07 & $\begin{array}{l}p= \\
0.20\end{array}$ & $x$ \\
\hline & & 1 & 29.17 & 3.87 & $\begin{array}{c}0.21 \\
(0.34) \\
\end{array}$ & 0.62 & $\begin{array}{c}-0.35 \\
(0.66)\end{array}$ & -0.53 & 0.07 & $\begin{array}{c}p= \\
0.20\end{array}$ & $x$ \\
\hline & & 2 & 26.23 & 5.16 & $\begin{array}{c}-0.17 \\
(0.47)\end{array}$ & -0.36 & $\begin{array}{c}-0.99 \\
(0.92)\end{array}$ & -1.08 & 0.12 & $\begin{array}{l}p= \\
0.20\end{array}$ & $x$ \\
\hline & \multirow{3}{*}{ Loss } & All & 17.92 & 5.79 & $\begin{array}{c}0.58 \\
(0.28) \\
\end{array}$ & 2.07 & $\begin{array}{c}-0.01 \\
(0.55)\end{array}$ & -0.02 & 0.10 & $\begin{array}{l}p= \\
0.05\end{array}$ & $x$ \\
\hline & & 1 & 17.22 & 6.20 & $\begin{array}{c}0.68 \\
(0.34) \\
\end{array}$ & 2.00 & $\begin{array}{c}-0.06 \\
(0.66)\end{array}$ & -0.09 & 0.14 & $\begin{array}{c}\mathrm{p}= \\
0.02\end{array}$ & $x$ \\
\hline & & 2 & 19.37 & 4.62 & $\begin{array}{r}1.01 \\
(0.47) \\
\end{array}$ & 2.15 & $\begin{array}{r}0.66 \\
(0.92) \\
\end{array}$ & 0.72 & 0.22 & $\begin{array}{c}p= \\
0.004\end{array}$ & $x$ \\
\hline & \multirow{3}{*}{ Physical } & All & 25.03 & 4.83 & $\begin{array}{c}-0.77 \\
(0.28) \\
\end{array}$ & -2.75 & $\begin{array}{l}-0.25 \\
(0.55) \\
\end{array}$ & -0.45 & 0.05 & $\begin{array}{c}p= \\
0.20\end{array}$ & $x$ \\
\hline & & 1 & 26.62 & 4.19 & $\begin{array}{c}-0.03 \\
(0.34) \\
\end{array}$ & -0.09 & $\begin{array}{c}-0.39 \\
(0.66) \\
\end{array}$ & -0.59 & 0.07 & $\begin{array}{c}p= \\
0.20\end{array}$ & $x$ \\
\hline & & 2 & 21.72 & 4.43 & $\begin{array}{c}0.13 \\
(0.47) \\
\end{array}$ & 0.28 & $\begin{array}{c}0.30 \\
(0.92) \\
\end{array}$ & 0.33 & 0.14 & $\begin{array}{c}p= \\
0.20\end{array}$ & $x$ \\
\hline \multirow{3}{*}{\multicolumn{2}{|c|}{ RAME }} & All & 28.31 & 7.14 & $\begin{array}{c}-0.67 \\
(0.28) \\
\end{array}$ & -2.39 & $\begin{array}{c}0.53 \\
(0.55) \\
\end{array}$ & 0.96 & 0.12 & $\begin{array}{l}p= \\
0.02\end{array}$ & $x$ \\
\hline & & 1 & 26.50 & 7.55 & $\begin{array}{c}-0.43 \\
(0.34) \\
\end{array}$ & -1.26 & $\begin{array}{c}-0.06 \\
(0.67) \\
\end{array}$ & -0.09 & 0.08 & $\begin{array}{c}\mathrm{p}= \\
0.20\end{array}$ & $x$ \\
\hline & & 2 & 32.07 & 4.31 & $\begin{array}{c}0.45 \\
(0.47) \\
\end{array}$ & 0.96 & $\begin{array}{c}0.98 \\
(0.92) \\
\end{array}$ & 1.07 & 0.12 & $\begin{array}{c}p= \\
0.20\end{array}$ & $x$ \\
\hline
\end{tabular}




\begin{tabular}{|c|c|c|c|c|c|c|c|c|c|c|c|}
\hline \multicolumn{2}{|c|}{ Measure } & Group* & Mean & SD & $\begin{array}{l}\text { Skew } \\
\text { (std. } \\
\text { error) }\end{array}$ & $\begin{array}{c}\mathbf{Z} \\
\text { Score }^{+}\end{array}$ & $\begin{array}{l}\text { Kurt } \\
\text { (std. } \\
\text { error) }\end{array}$ & $\begin{array}{c}\mathbf{Z} \\
\text { Score+ }\end{array}$ & $\mathbf{K}_{-} \mathbf{S}^{++}$ & $\begin{array}{c}p \\
\text { value }\end{array}$ & $\begin{array}{l}\text { Signif. } \\
\text { deviat. }\end{array}$ \\
\hline \multirow{3}{*}{\multicolumn{2}{|c|}{ GHQ-12 }} & All & 12.33 & 6.38 & $\begin{array}{c}0.84 \\
(0.28)\end{array}$ & 3.00 & $\begin{array}{l}-0.06 \\
(0.55)\end{array}$ & -0.11 & 0.16 & $\begin{array}{c}\mathrm{p}< \\
0.001\end{array}$ & $\checkmark$ \\
\hline & & 1 & 10.39 & 5.40 & $\begin{array}{c}1.34 \\
(0.34)\end{array}$ & 3.94 & $\begin{array}{c}2.07 \\
(0.66)\end{array}$ & 3.14 & 0.21 & $\begin{array}{c}\mathrm{p}< \\
0.001\end{array}$ & $\checkmark$ \\
\hline & & 2 & 16.39 & 6.45 & $\begin{array}{c}0.16 \\
(0.47) \\
\end{array}$ & 0.34 & $\begin{array}{l}-0.97 \\
(0.92)\end{array}$ & -1.05 & 0.14 & $\begin{array}{c}\mathrm{p}= \\
0.20\end{array}$ & $x$ \\
\hline \multirow{9}{*}{ RHBC } & \multirow{3}{*}{ Composite } & All & 69.88 & 9.80 & $\begin{array}{c}-0.07 \\
(0.28)\end{array}$ & -0.25 & $\begin{array}{l}-0.45 \\
(0.55)\end{array}$ & -0.82 & 0.11 & $\begin{array}{c}\mathrm{p}= \\
0.02\end{array}$ & $x$ \\
\hline & & 1 & 72.72 & 8.90 & $\begin{array}{c}-0.16 \\
(0.34)\end{array}$ & -0.47 & $\begin{array}{c}-0.23 \\
(0.66)\end{array}$ & -0.35 & 0.10 & $\begin{array}{c}\mathrm{p}= \\
0.20\end{array}$ & $x$ \\
\hline & & 2 & 63.96 & 9.05 & $\begin{array}{l}-0.01 \\
(0.47)\end{array}$ & -0.02 & $\begin{array}{l}-1.44 \\
(0.92)\end{array}$ & -1.57 & 0.16 & $\begin{array}{l}\mathrm{p}= \\
0.13\end{array}$ & $x$ \\
\hline & \multirow{3}{*}{$\begin{array}{l}\text { Health- } \\
\text { Action }\end{array}$} & All & 46.06 & 7.81 & $\begin{array}{l}-0.25 \\
(0.28)\end{array}$ & -0.89 & $\begin{array}{l}-0.47 \\
(0.55)\end{array}$ & -0.85 & 0.11 & $\begin{array}{c}\mathrm{p}= \\
0.02\end{array}$ & $x$ \\
\hline & & 1 & 47.37 & 7.63 & $\begin{array}{l}-0.27 \\
(0.34)\end{array}$ & -0.79 & $\begin{array}{c}0.34 \\
(0.66)\end{array}$ & -0.52 & 0.10 & $\begin{array}{c}\mathrm{p}= \\
0.20\end{array}$ & $x$ \\
\hline & & 2 & 43.34 & 7.65 & $\begin{array}{l}-0.30 \\
(0.47)\end{array}$ & -0.64 & $\begin{array}{l}-0.88 \\
(0.92)\end{array}$ & -0.96 & 0.14 & $\begin{array}{c}\mathrm{p}= \\
0.20\end{array}$ & $x$ \\
\hline & \multirow{3}{*}{$\begin{array}{l}\text { Cognitive- } \\
\text { Affective }\end{array}$} & All & 23.82 & 4.28 & $\begin{array}{c}-1.18 \\
(0.28) \\
\end{array}$ & -4.21 & $\begin{array}{l}2.95 \\
(0.55) \\
\end{array}$ & 5.36 & 0.14 & $\begin{array}{c}\mathrm{p}= \\
0.001\end{array}$ & Border \\
\hline & & 1 & 25.35 & 3.10 & $\begin{array}{l}-0.36 \\
(0.34)\end{array}$ & -1.06 & $\begin{array}{l}-0.12 \\
(0.66)\end{array}$ & -0.18 & 0.13 & $\begin{array}{c}\mathrm{p}= \\
0.04\end{array}$ & $x$ \\
\hline & & 2 & 20.62 & 4.67 & $\begin{array}{c}-1.30 \\
(0.47)\end{array}$ & -2.77 & $\begin{array}{c}2.71 \\
(0.92)\end{array}$ & 2.95 & 0.16 & $\begin{array}{c}\mathrm{p}= \\
0.12\end{array}$ & $x$ \\
\hline
\end{tabular}

* All = total sample $(n=74) ; 1=$ non-clinical sample $(n=50) ; 2=$ clinical sample $(n=24)$

+ Absolute value greater than 3.29 is significant at $p<0.001$ level. Significant values highlighted in bold.

$++\mathrm{K}-\mathrm{S}=$ Kolmogorov-Smirnov one-sample test which tests whether the distribution as a whole deviates from a normal distribution; $\mathrm{df}=74$ (whole sample); $\mathrm{df}=50$ (Group1); $\mathrm{df}=24$ (Group 2). Alpha set at $\mathrm{p}<0.001$ for multiple tests of normality. 


\section{APPENDIX 4.5: HISTOGRAMS OF SCALE DISTRIBUTIONS}

BMHSS: Ageism

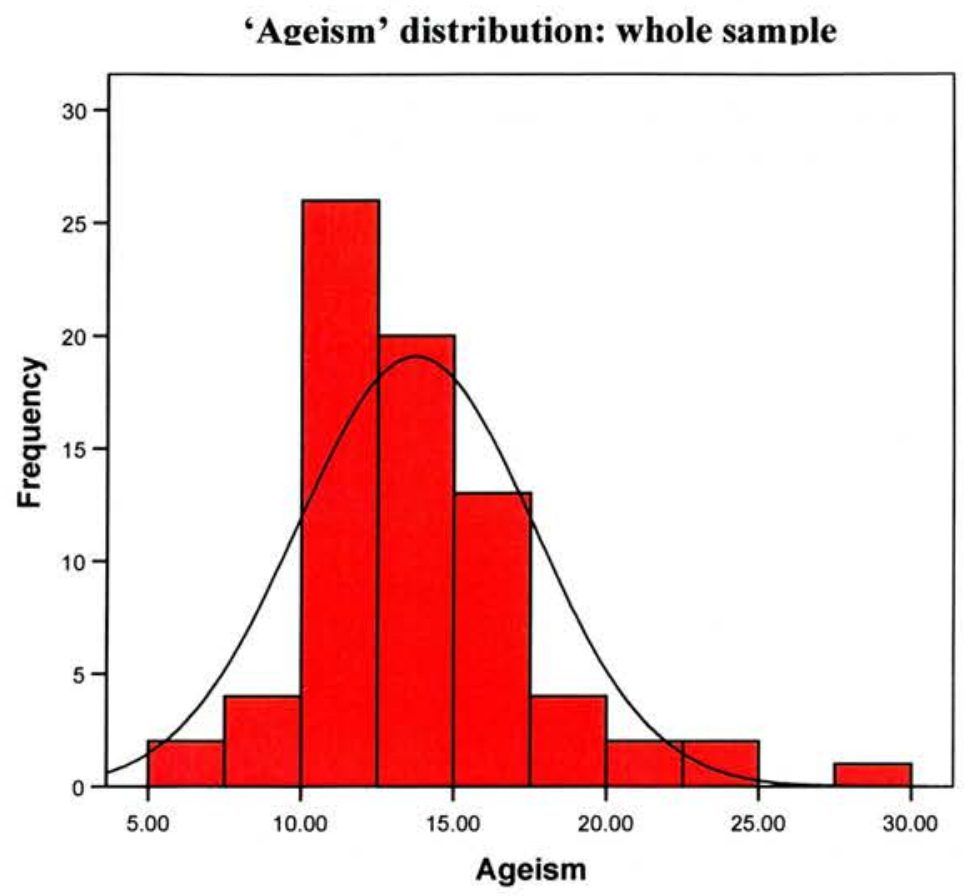

Mean $=13.739$ Std. Dev. $=3.86565$

BMHSS: Stigma

'Stigma' distribution: whole sample

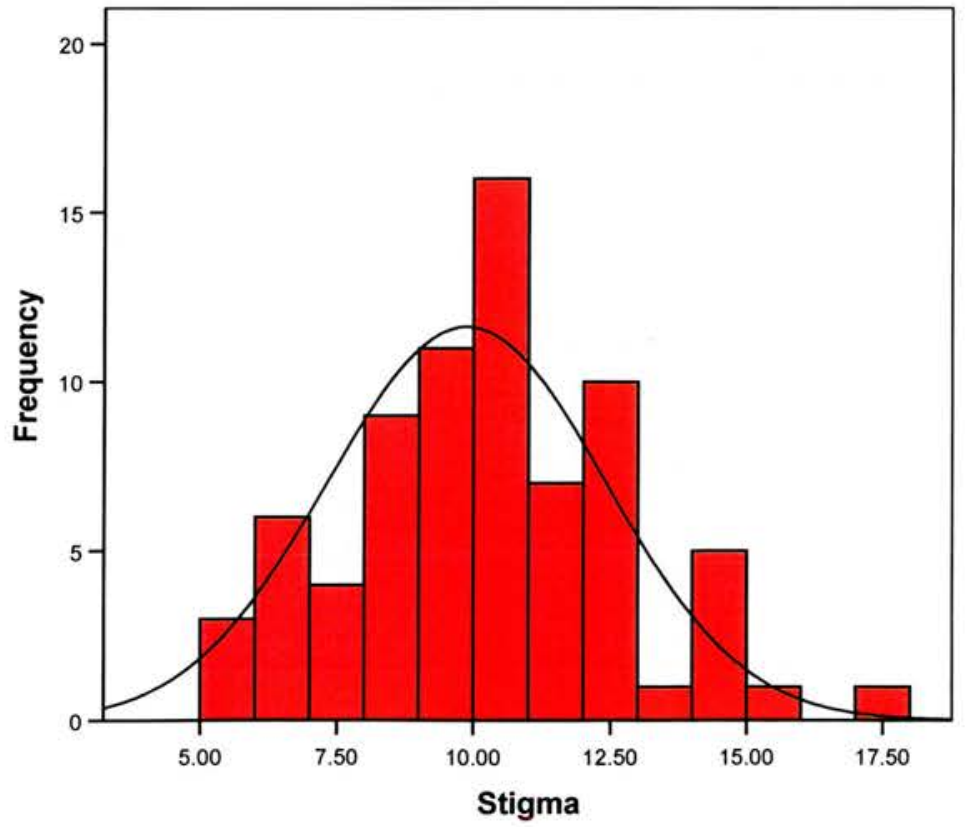

Mean $=9.8659$

Std. Dev. $=2.53765$ $\mathrm{N}=74$ 


\section{Understandability}

Understandability distribution: whole sample

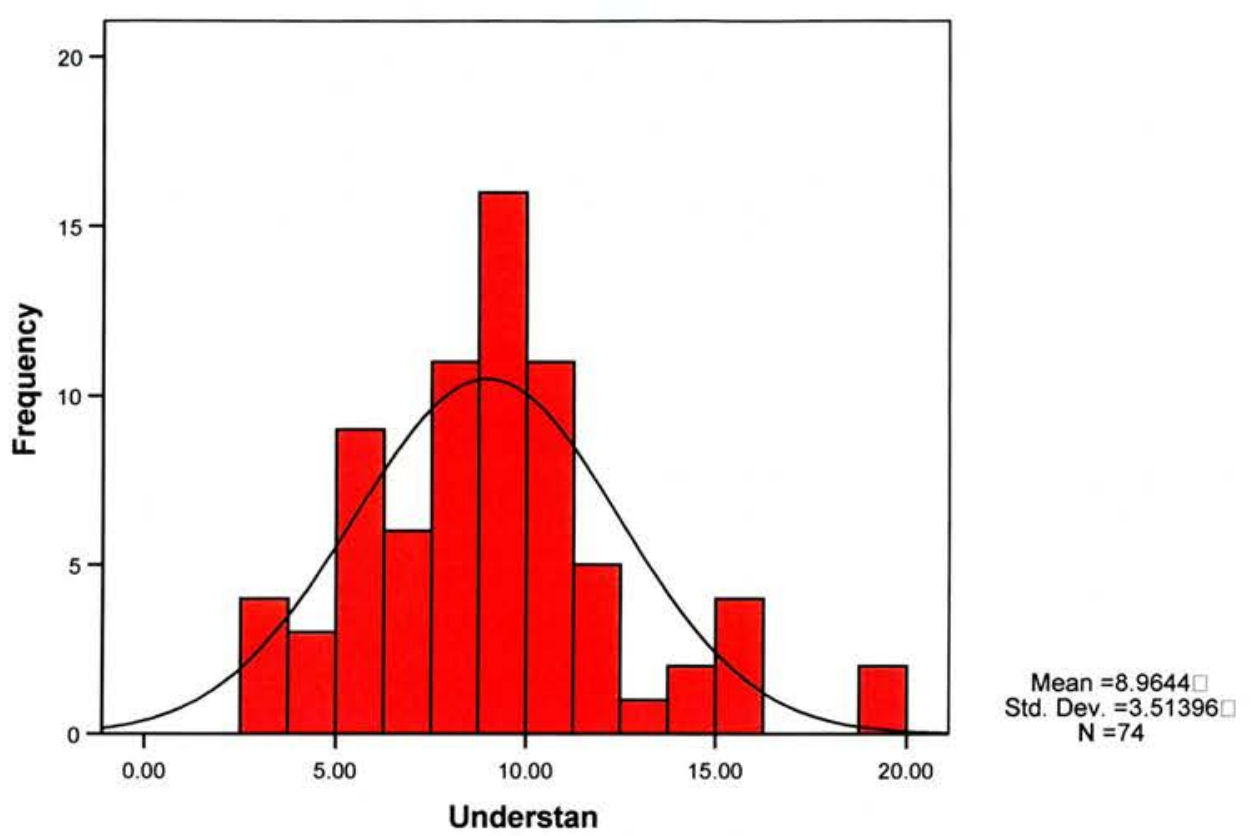




\section{ATTITUDES TO AGEING}

\section{AAQ: Composite}

AAO distribution: whole sample

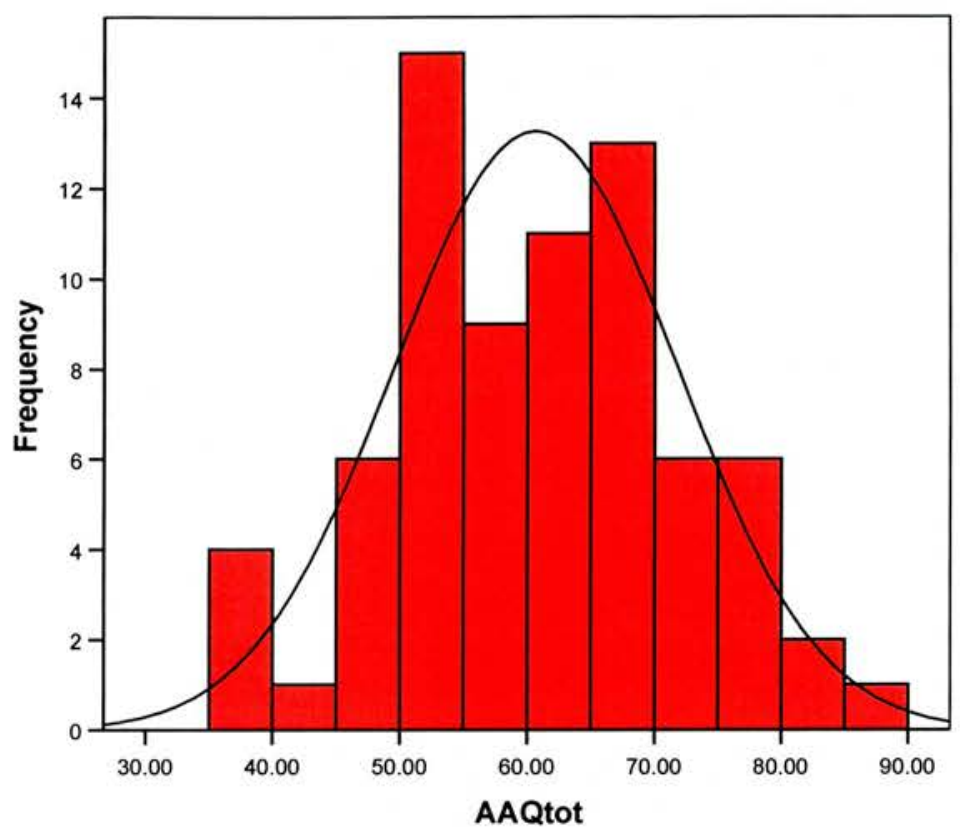

Mean $=60.6751$ Std. Dev. $=11.12659$

AAQ: Growth

'Growth' distribution: whole sample

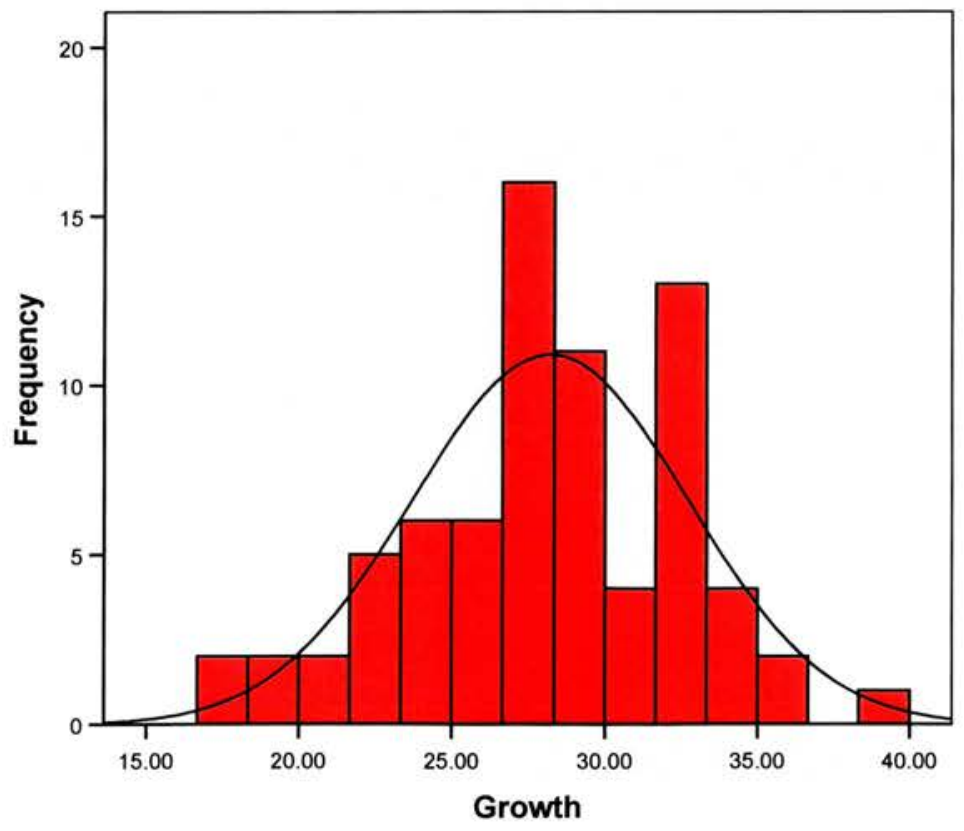

Mean $=28.2119$ Std. Dev. $=4.51383$ $\mathrm{N}=74$ 


\section{AAQ: Loss}

'Loss' distribution: whole sample

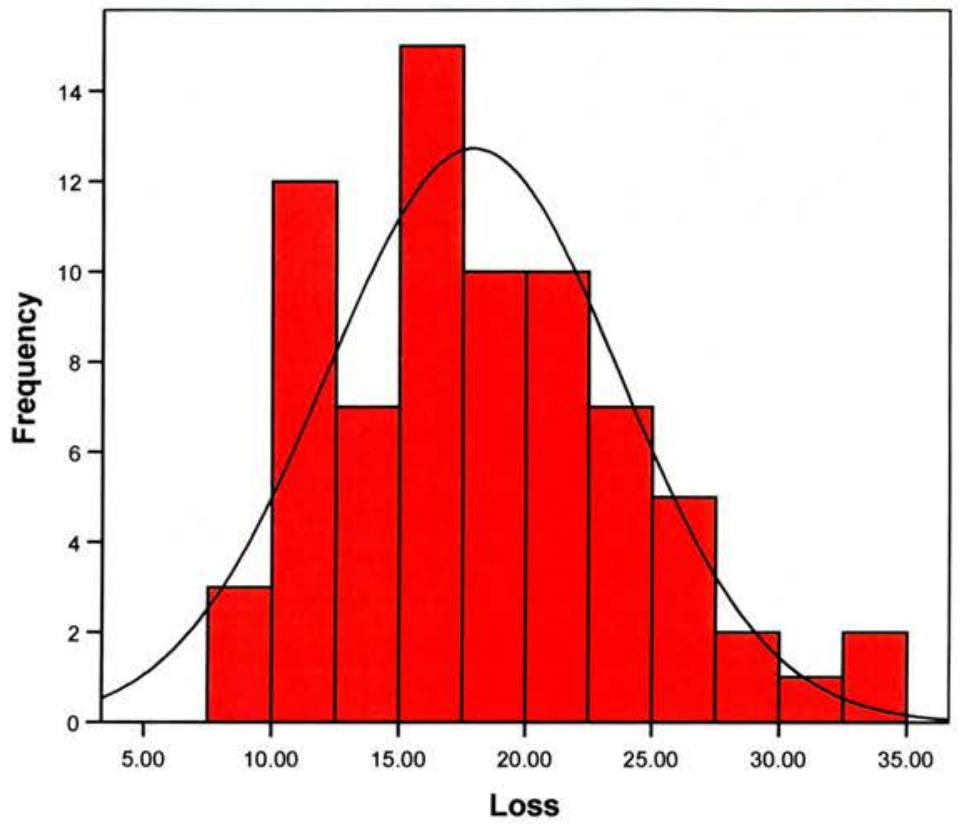

Mean $=17.9161$ $\begin{aligned} \text { Std. Dev. } & =5.79418 \\ N & =74\end{aligned}$

\section{AAQ: Physical}

'Phvsical' distribution: whole sample

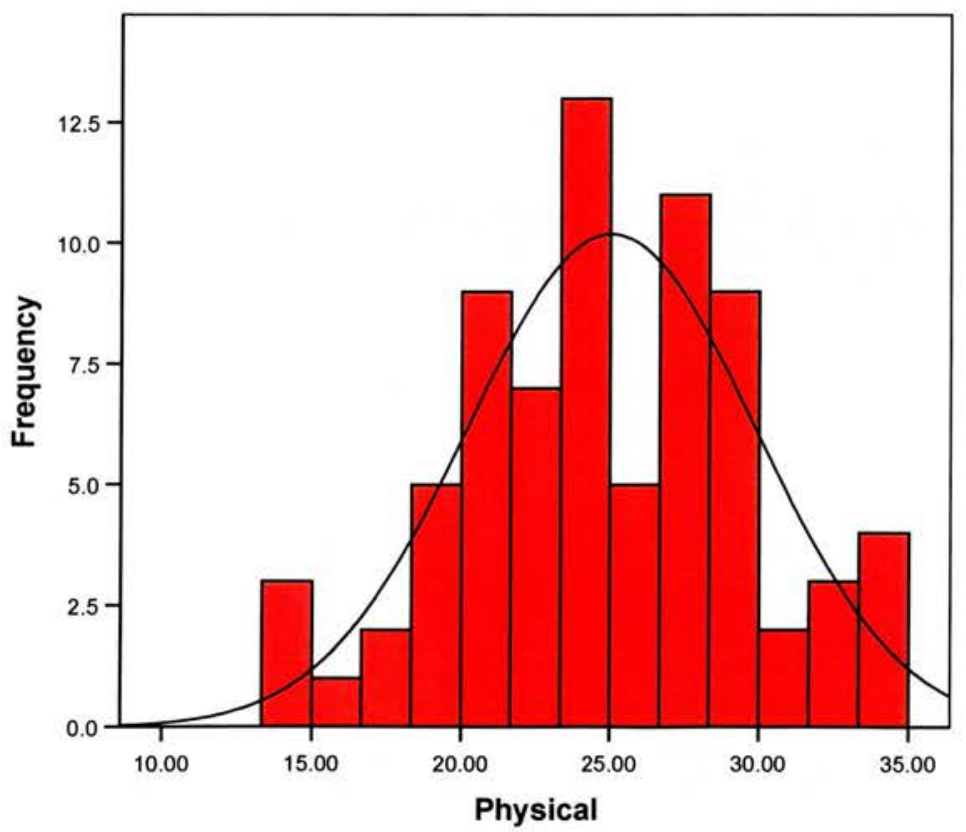

Mean $=25.0291$ Std. Dev. $=4.82611 \mathrm{C}$ 


\section{RAME}

\section{Rame distribution: whole sample}

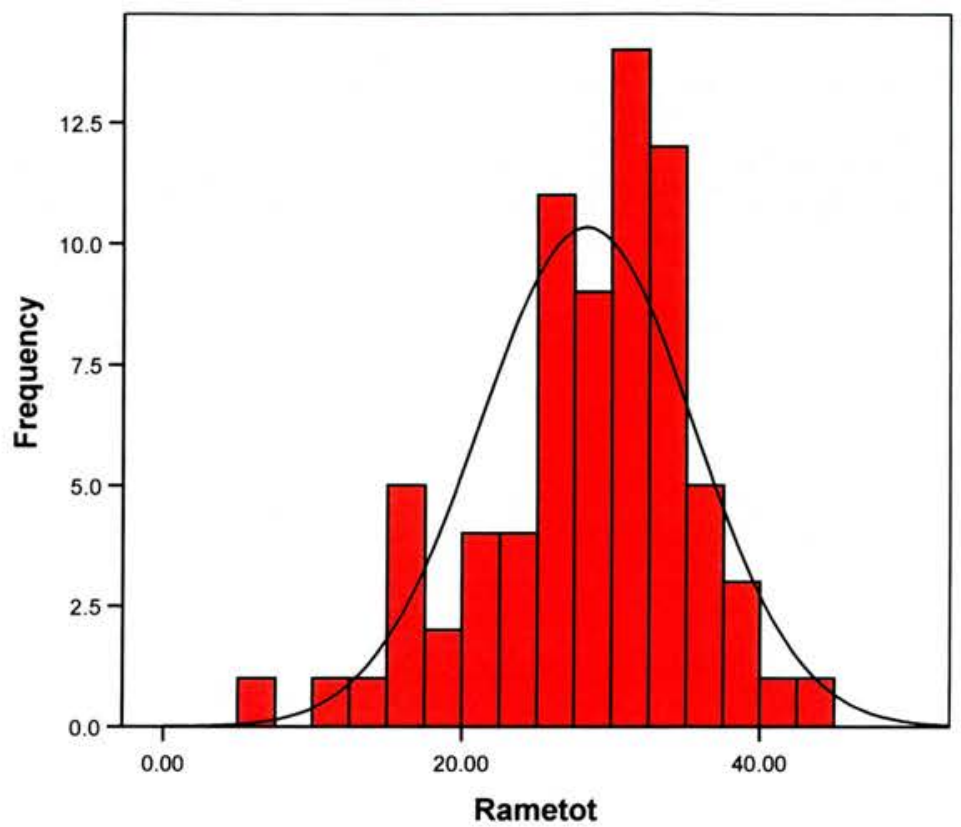

Mean $=28.3073$ Std. Dev. $=7.14008$ $\mathrm{N}=74$

\section{GHQ-12}

\section{GHO-12 distribution: whole sample}

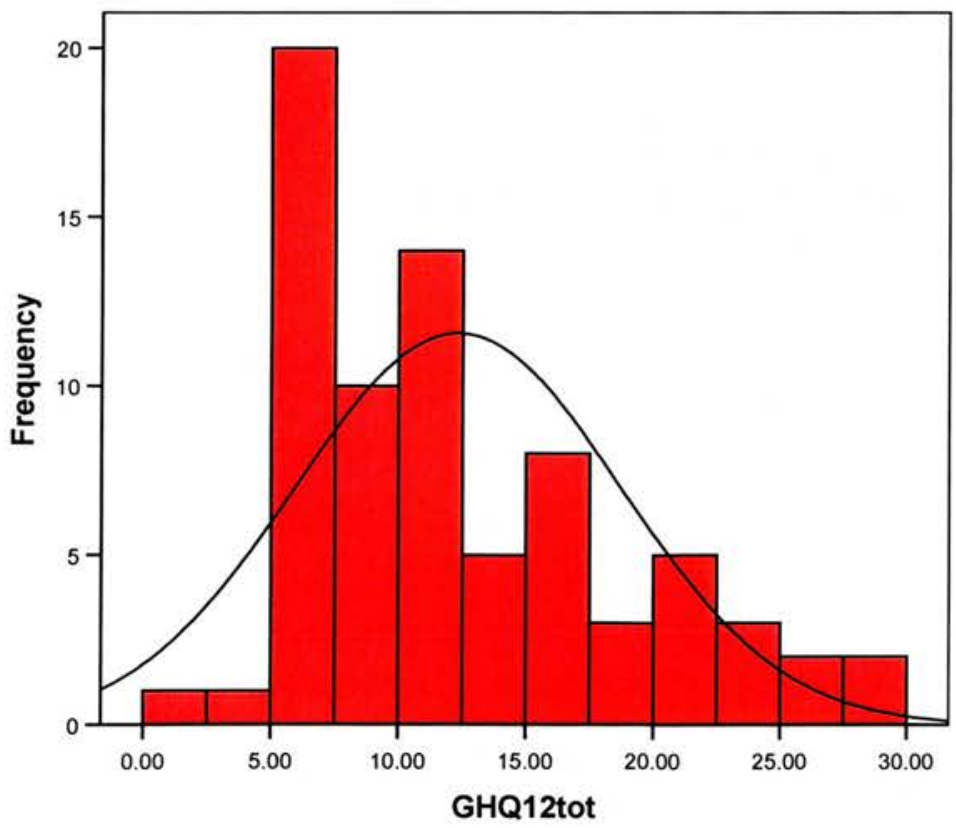

Mean $=12.3327$ $\begin{aligned} \text { Std. Dev. } & =6.37894 \\ N & =74\end{aligned}$ 


\section{RHBC: Composite}

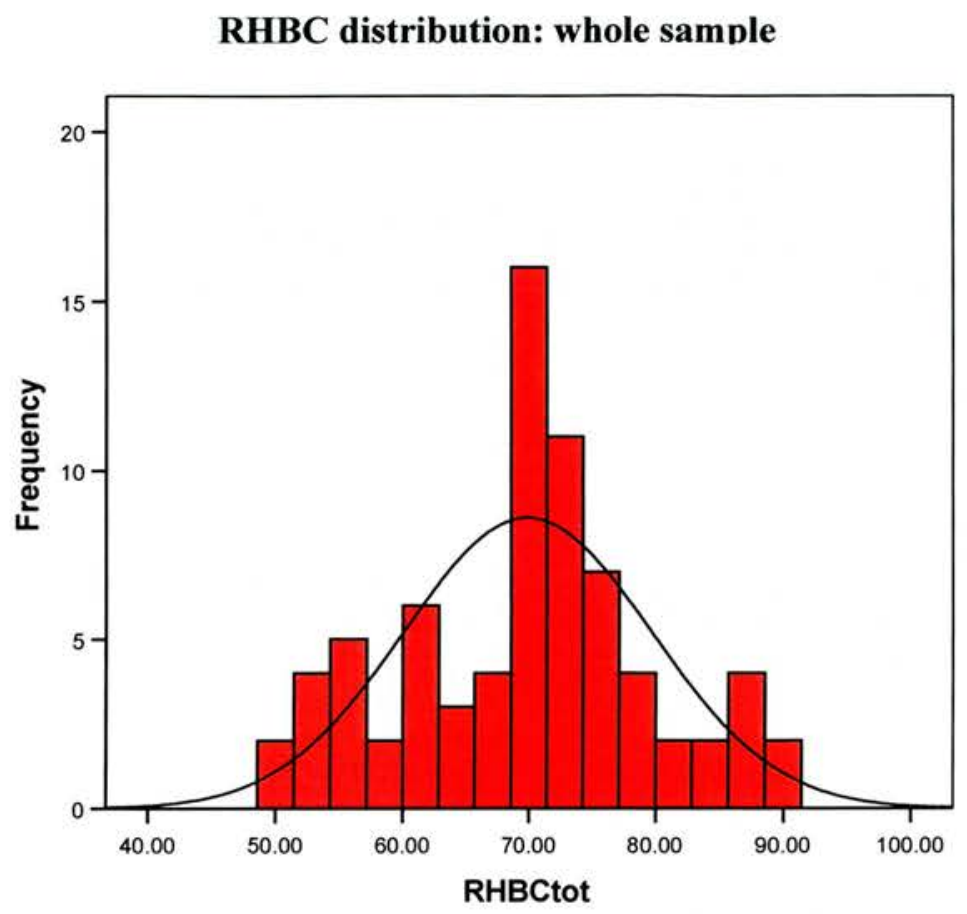

Mean $=69.8808$

\section{RHBC: Health Action}

'Health Action' distribution: whole sample

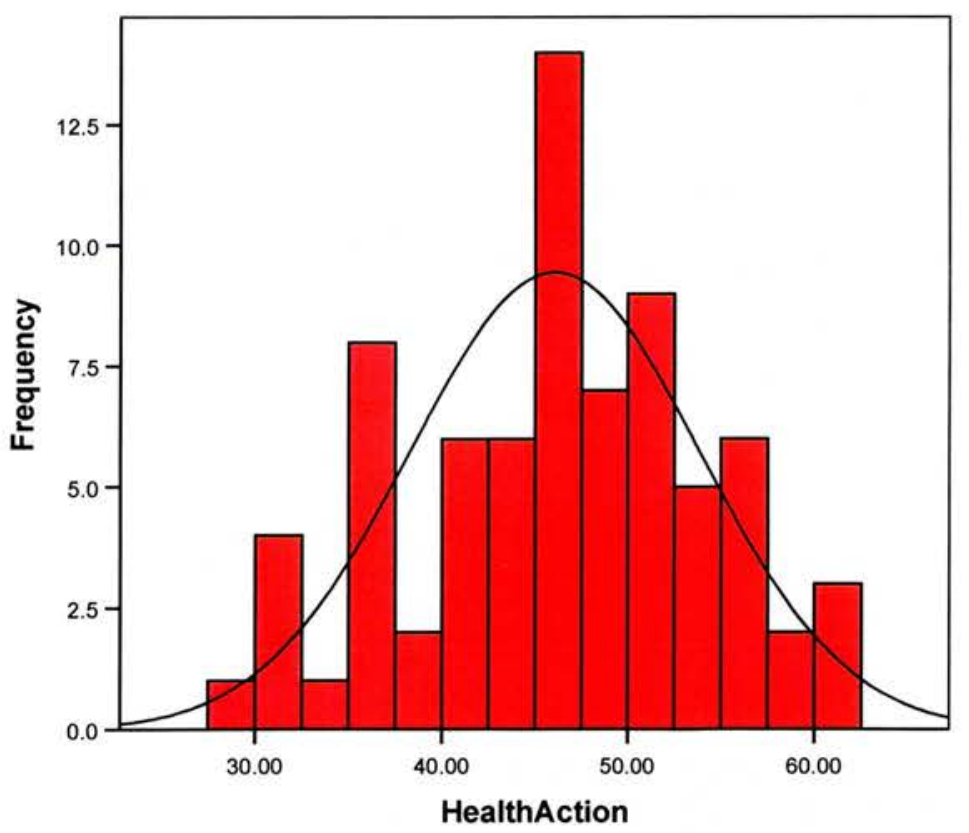

Mean $=46.063$

Std. Dev. $=7.81341$ $\mathrm{N}=74$ 


\section{RHBC: Cognitive Affective}

'Cognitive Affective' distribution: whole sample

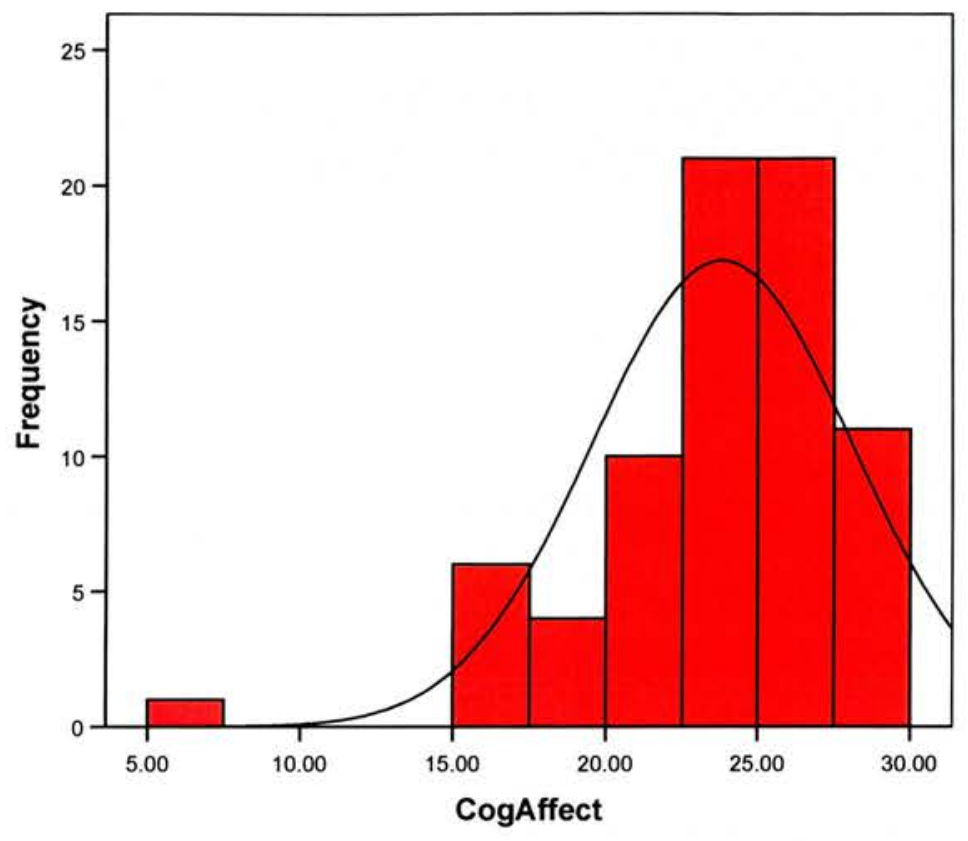

Mean $=23,8178$ Std. Dev. $=4.28095$
$N=74$ 
APPENDIX 4.6: TESTS OF HOMOGENEITY OF VARIANCE FOR COMPUTED SCALES (WHOLE SAMPLE)

\section{BMHSS: Ageism}

\begin{tabular}{|ll|r|r|r|r|}
\hline & $\begin{array}{c}\text { Levene } \\
\text { Statistic }\end{array}$ & df1 & df2 & \multicolumn{1}{c|}{ Sig. } \\
\hline Ageism & Based on Mean & .805 & 1 & 72 & .373 \\
& Based on Median & .744 & 1 & 72 & .391 \\
& Based on Median and with & .744 & 1 & 71.988 & .391 \\
adjusted df & .839 & 1 & 72 & .363 \\
Based on trimmed mean & & & & \\
\end{tabular}

\section{BMHSS: Stigma}

\begin{tabular}{|ll|r|r|r|r|}
\hline & \multicolumn{1}{|c|}{$\begin{array}{l}\text { Levene } \\
\text { Statistic }\end{array}$} & df1 & df2 & \multicolumn{1}{c|}{ Sig. } \\
\hline Stigma & Based on Mean & .423 & 1 & 72 & .517 \\
& Based on Median & .249 & 1 & 72 & .619 \\
& Based on Median and with & .249 & 1 & 71.820 & .619 \\
& adjusted df & .394 & 1 & 72 & .532 \\
& Based on trimmed mean & & & & \\
\hline
\end{tabular}

\section{Understandability}

\begin{tabular}{|ll|r|r|r|r|}
\hline & \multicolumn{1}{|c|}{$\begin{array}{l}\text { Levene } \\
\text { Statistic }\end{array}$} & df1 & df2 & \multicolumn{1}{c|}{ Sig. } \\
\hline Understan & Based on Mean & 2.759 & 1 & 72 & .101 \\
& Based on Median & 2.535 & 1 & 72 & .116 \\
& Based on Median and with & 2.535 & 1 & 65.127 & .116 \\
& adjusted df & 2.734 & 1 & 72 & .103 \\
\hline
\end{tabular}

\section{AAQ: Total Score}

\begin{tabular}{|ll|r|r|r|r|}
\hline & $\begin{array}{c}\text { Levene } \\
\text { Statistic }\end{array}$ & df1 & df2 & \multicolumn{1}{c|}{ Sig. } \\
\hline AAQtot & Based on Mean & .054 & 1 & 72 & .816 \\
& Based on Median & .068 & 1 & 72 & .796 \\
& Based on Median and with & .068 & 1 & 71.701 & .796 \\
adjusted df & .055 & 1 & 72 & .815 \\
Based on trimmed mean & & 1 & & \\
\hline
\end{tabular}




\section{AAQ: Subscales}

\begin{tabular}{|c|c|c|c|c|c|}
\hline & & $\begin{array}{l}\text { Levene } \\
\text { Statistic }\end{array}$ & df1 & df2 & Sig. \\
\hline \multirow[t]{4}{*}{ Growth } & Based on Mean & 3.246 & 1 & 72 & .076 \\
\hline & Based on Median & 2.867 & 1 & 72 & .095 \\
\hline & $\begin{array}{l}\text { Based on Median and with } \\
\text { adjusted df }\end{array}$ & 2.867 & 1 & 66.378 & .095 \\
\hline & Based on trimmed mean & 3.233 & 1 & 72 & .076 \\
\hline \multirow[t]{4}{*}{ Loss } & Based on Mean & 3.283 & 1 & 72 & .074 \\
\hline & Based on Median & 3.080 & 1 & 72 & .084 \\
\hline & $\begin{array}{l}\text { Based on Median and with } \\
\text { adjusted df }\end{array}$ & 3.080 & 1 & 70.649 & .084 \\
\hline & Based on trimmed mean & 3.304 & 1 & 72 & .073 \\
\hline \multirow[t]{4}{*}{ Physical } & Based on Mean & .002 & 1 & 72 & .966 \\
\hline & Based on Median & .000 & 1 & 72 & .985 \\
\hline & $\begin{array}{l}\text { Based on Median and with } \\
\text { adjusted df }\end{array}$ & .000 & 1 & 70.980 & .985 \\
\hline & Based on trimmed mean & .002 & 1 & 72 & .966 \\
\hline
\end{tabular}

\section{Rame}

\begin{tabular}{|ll|r|r|r|r|}
\hline & \multicolumn{1}{|c|}{$\begin{array}{c}\text { Levene } \\
\text { Statistic }\end{array}$} & \multicolumn{1}{c|}{ df1 } & \multicolumn{1}{c|}{ df2 } & \multicolumn{1}{c|}{ Sig. } \\
\hline Rametot & Based on Mean & 7.902 & 1 & 72 & .006 \\
& Based on Median & 7.374 & 1 & 72 & .008 \\
& Based on Median and with & 7.374 & 1 & 63.865 & .009 \\
adjusted df & 7.840 & 1 & 72 & .007 \\
\hline
\end{tabular}

\section{GHQ:12}

\begin{tabular}{|ll|r|r|r|r|}
\hline & & Levene & & & \\
\hline Statistic & df1 & df2 & \multicolumn{1}{c|}{ Sig. } \\
\hline & 2.643 & 1 & 72 & .108 \\
& 2.843 & 1 & 72 & .096 \\
& Based on Median & 2.843 & 1 & 71.142 & .096 \\
& Based on Median and with & 2.962 & 1 & 72 & .090 \\
\hline
\end{tabular}

\section{RHBC: Total Score}

\begin{tabular}{|ll|r|r|r|r|}
\hline & \multicolumn{1}{|c|}{$\begin{array}{c}\text { Levene } \\
\text { Statistic }\end{array}$} & \multicolumn{1}{c|}{ df1 } & \multicolumn{1}{c|}{ df2 } & \multicolumn{1}{c|}{ Sig. } \\
\hline RHBCtot & Based on Mean & .778 & 1 & 72 & .381 \\
& Based on Median & .821 & 1 & 72 & .368 \\
& Based on Median and with & .821 & 1 & 68.681 & .368 \\
& adjusted df & & 1 & 72 & .385 \\
\hline
\end{tabular}




\section{RHBC: Subscales}

\begin{tabular}{|c|c|c|c|c|c|}
\hline & & $\begin{array}{l}\text { Levene } \\
\text { Statistic }\end{array}$ & df1 & $\mathrm{df} 2$ & Sig. \\
\hline \multirow[t]{4}{*}{ HealthAction } & Based on Mean & .318 & 1 & 72 & .575 \\
\hline & Based on Median & .267 & 1 & 72 & .607 \\
\hline & $\begin{array}{l}\text { Based on Median and with } \\
\text { adjusted df }\end{array}$ & .267 & 1 & 71.368 & .607 \\
\hline & Based on trimmed mean & .280 & 1 & 72 & .598 \\
\hline \multirow[t]{4}{*}{ CogAffect } & Based on Mean & 4.229 & 1 & 72 & .043 \\
\hline & Based on Median & 2.602 & 1 & 72 & .111 \\
\hline & $\begin{array}{l}\text { Based on Median and with } \\
\text { adjusted df }\end{array}$ & 2.602 & 1 & 49.702 & .113 \\
\hline & Based on trimmed mean & 3.585 & 1 & 72 & .062 \\
\hline
\end{tabular}




\section{APPENDIX 4.7: RELIABILITY ANALYSIS OF SCALES}

\section{BMHSS: Stigma}

Reliability Statistics

\begin{tabular}{|c|c|c|}
\hline $\begin{array}{c}\text { Cronbach's } \\
\text { Alpha }\end{array}$ & $\begin{array}{c}\text { Cronbach's } \\
\text { Alpha Based } \\
\text { on } \\
\text { Standardized } \\
\text { Items } \\
\end{array}$ & $\mathrm{N}$ of Items \\
\hline .511 & .536 & 5 \\
\hline
\end{tabular}

Item-Total Statistics

\begin{tabular}{|l|r|r|r|r|r|}
\hline & $\begin{array}{c}\text { Scale Mean if } \\
\text { Item Deleted }\end{array}$ & $\begin{array}{c}\text { Scale } \\
\text { Variance if } \\
\text { Item Deleted }\end{array}$ & $\begin{array}{c}\text { Corrected } \\
\text { Item-Total } \\
\text { Correlation }\end{array}$ & $\begin{array}{c}\text { Squared } \\
\text { Multiple } \\
\text { Correlation }\end{array}$ & $\begin{array}{c}\text { Cronbach's } \\
\text { Alpha if Item } \\
\text { Deleted }\end{array}$ \\
\hline SMEAN(BMHSS2) & 7.9704 & 4.061 & .470 & .308 & .327 \\
SMEAN(BMHSS4) & 8.1747 & 4.414 & .339 & .169 & .417 \\
SMEAN(bmhsS6R) & 7.3810 & 5.201 & .054 & .021 & .612 \\
SMEAN(BMHSS8) & 8.0865 & 4.531 & .435 & .256 & .373 \\
SMEAN(BMHSS11) & 7.8510 & 4.919 & .199 & .070 & .505 \\
\hline
\end{tabular}

\section{UNDERSTANDABILITY}

\section{Reliability Statistics}

\begin{tabular}{|c|c|c|}
\hline $\begin{array}{l}\text { Cronbach's } \\
\text { Alpha }\end{array}$ & $\begin{array}{c}\text { Cronbach's } \\
\text { Alpha Based } \\
\text { on } \\
\text { Standardized } \\
\text { Items }\end{array}$ & $\mathrm{N}$ of Items \\
\hline .388 & .413 & 3 \\
\hline
\end{tabular}

Item-Total Statistics

\begin{tabular}{|l|r|r|r|r|r|}
\hline & $\begin{array}{c}\text { Scale Mean if } \\
\text { Item Deleted }\end{array}$ & $\begin{array}{c}\text { Scale } \\
\text { Variance if } \\
\text { Item Deleted }\end{array}$ & $\begin{array}{c}\text { Corrected } \\
\text { Item-Total } \\
\text { Correlation }\end{array}$ & $\begin{array}{c}\text { Squared } \\
\text { Multiple } \\
\text { Correlation }\end{array}$ & $\begin{array}{c}\text { Cronbach's } \\
\text { Alpha if Item } \\
\text { Deleted }\end{array}$ \\
\hline SMEAN(underst1R) & 4.4714 & 7.726 & .070 & .037 & .628 \\
SMEAN(underst2R) & 6.5501 & 5.971 & .409 & .241 & $-.081(\mathrm{a})$ \\
SMEAN(underst3R) & 6.9072 & 7.809 & .255 & .225 & .257 \\
\hline
\end{tabular}

a The value is negative due to a negative average covariance among items. This violates reliability model assumptions. You may want to check item codings. 


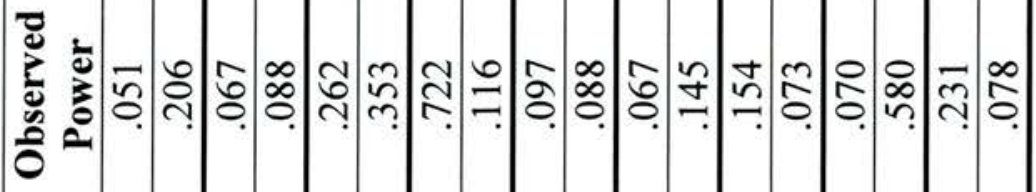

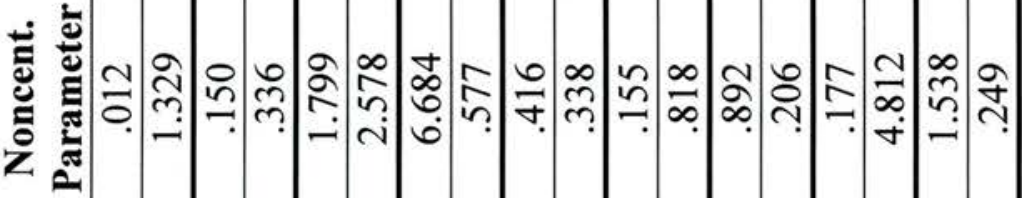

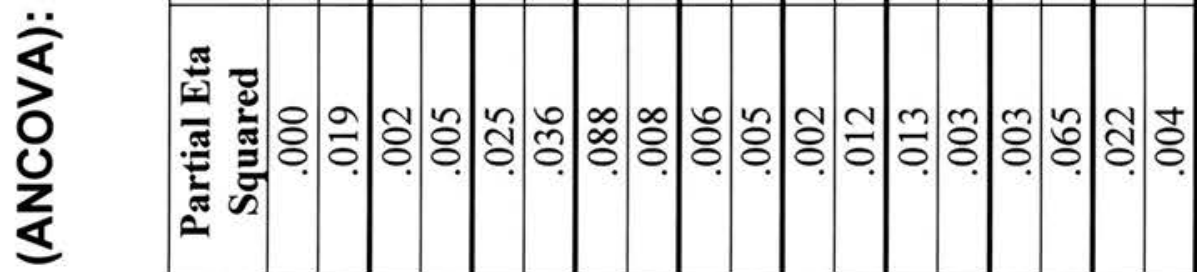

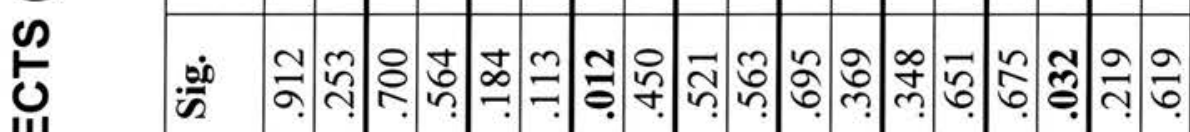

U1

岌

का

岂通

立

ш

$\sum$ U

岗

岁

の

귀유ำ

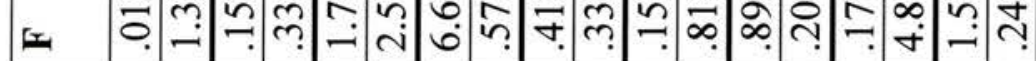

$\infty>$

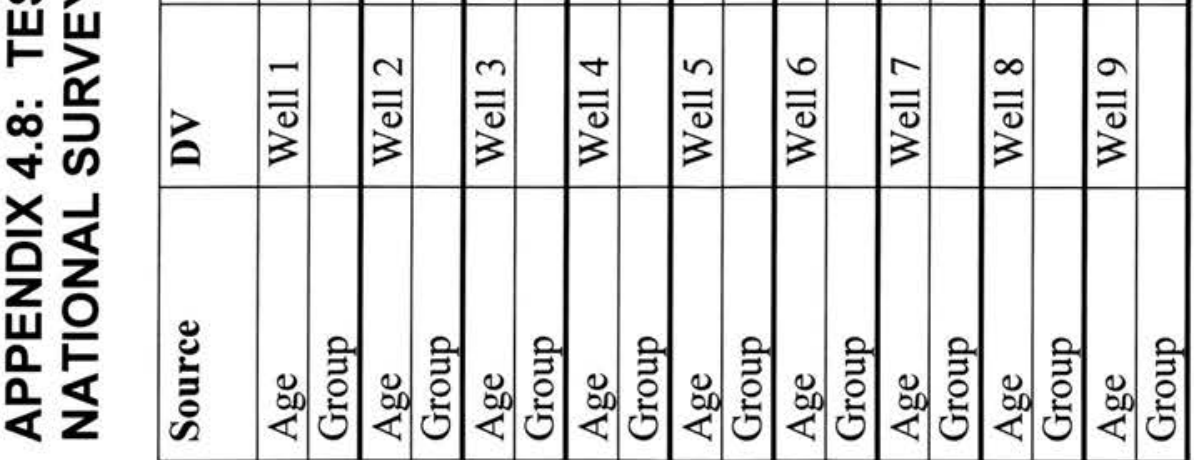




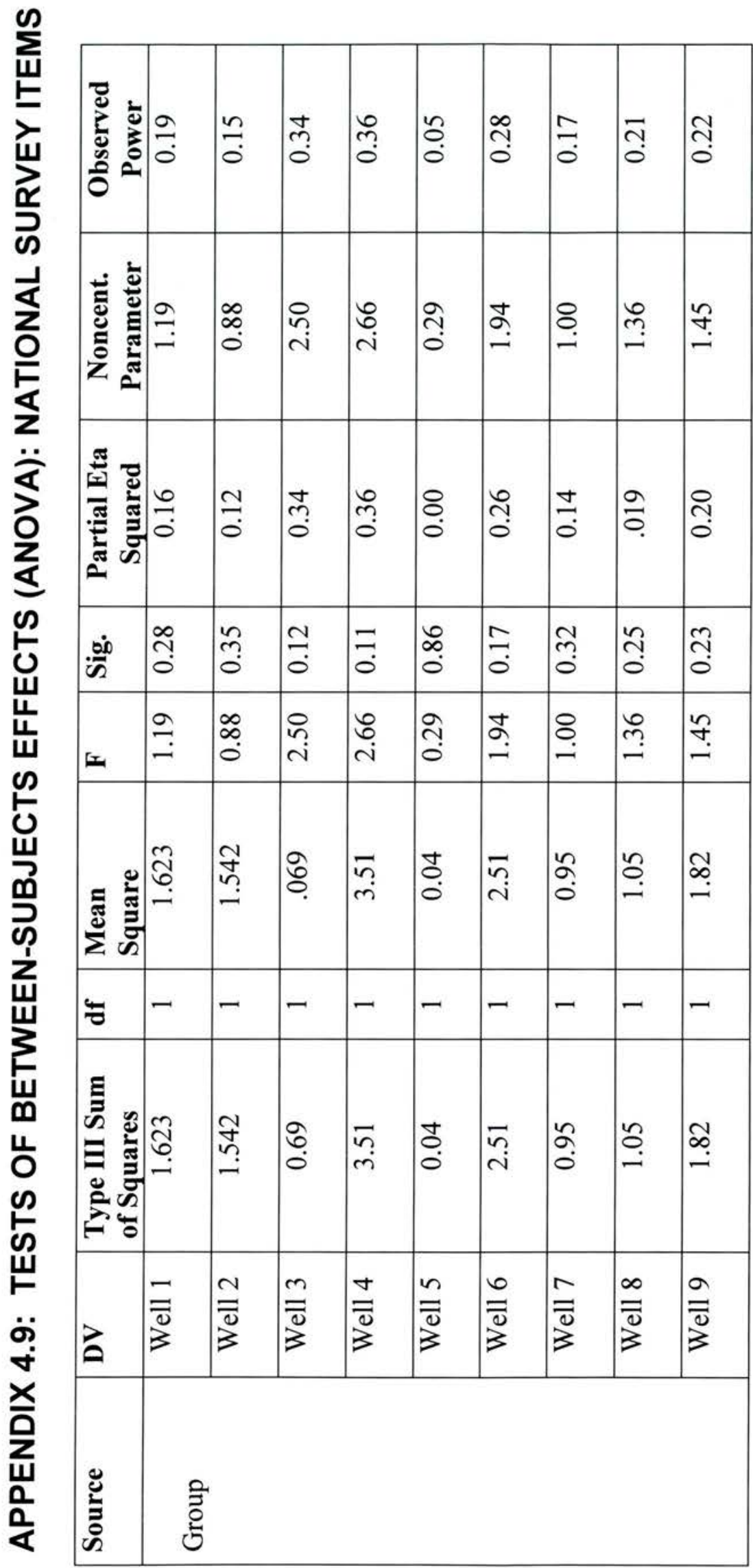




\section{APPENDIX 4.10: COMPARISON OF ATTITUDES TO MENTAL ILLNESS BY GENDER (NATIONAL SURVEY ITEM 1)}

Item 1: If I was suffering from mental health problems, I wouldn't want people knowing about it

\section{Crosstab}

\begin{tabular}{|c|c|c|c|c|c|}
\hline & & & \multicolumn{2}{|c|}{ Sex } & \multirow[b]{2}{*}{ Total } \\
\hline & & & Male & Female & \\
\hline \multirow[t]{8}{*}{ well $1 \mathrm{r}$} & 1.00 & Count & 16 & 14 & 30 \\
\hline & & $\%$ within well1 $r$ & $53.3 \%$ & $46.7 \%$ & $100.0 \%$ \\
\hline & & $\%$ within Sex & $76.2 \%$ & $48.3 \%$ & $60.0 \%$ \\
\hline & & $\%$ of Total & $32.0 \%$ & $28.0 \%$ & $60.0 \%$ \\
\hline & 2.00 & Count & 5 & 15 & 20 \\
\hline & & $\%$ within well $1 \mathrm{r}$ & $25.0 \%$ & $75.0 \%$ & $100.0 \%$ \\
\hline & & $\%$ within Sex & $23.8 \%$ & $51.7 \%$ & $40.0 \%$ \\
\hline & & $\%$ of Total & $10.0 \%$ & $30.0 \%$ & $40.0 \%$ \\
\hline \multirow[t]{4}{*}{ Total } & & Count & 21 & 29 & 50 \\
\hline & & $\%$ within well $1 r$ & $42.0 \%$ & $58.0 \%$ & $100.0 \%$ \\
\hline & & $\%$ within Sex & $100.0 \%$ & $100.0 \%$ & $100.0 \%$ \\
\hline & & $\%$ of Total & $42.0 \%$ & $58.0 \%$ & $100.0 \%$ \\
\hline
\end{tabular}

\section{Chi-Square Tests}

\begin{tabular}{|c|c|c|c|c|c|}
\hline & Value & df & $\begin{array}{l}\text { Asymp. Sig. } \\
\text { (2-sided) }\end{array}$ & $\begin{array}{l}\text { Exact Sig. } \\
\text { (2-sided) }\end{array}$ & $\begin{array}{l}\text { Exact Sig. } \\
\text { (1-sided) }\end{array}$ \\
\hline Pearson Chi-Square & $3.955(b)$ & 1 & .047 & \multirow{6}{*}{.079} & \multirow{6}{*}{.044} \\
\hline $\begin{array}{l}\text { Continuity } \\
\text { Correction(a) }\end{array}$ & 2.877 & 1 & .090 & & \\
\hline Likelihood Ratio & 4.080 & 1 & .043 & & \\
\hline Fisher's Exact Test & & & & & \\
\hline $\begin{array}{l}\text { Linear-by-Linear } \\
\text { Association }\end{array}$ & 3.875 & 1 & .049 & & \\
\hline $\mathrm{N}$ of Valid Cases & 50 & & & & \\
\hline
\end{tabular}

a Computed only for a $2 \times 2$ table

b 0 cells $(.0 \%)$ have expected count less than 5 . The minimum expected count is 8.40 . 


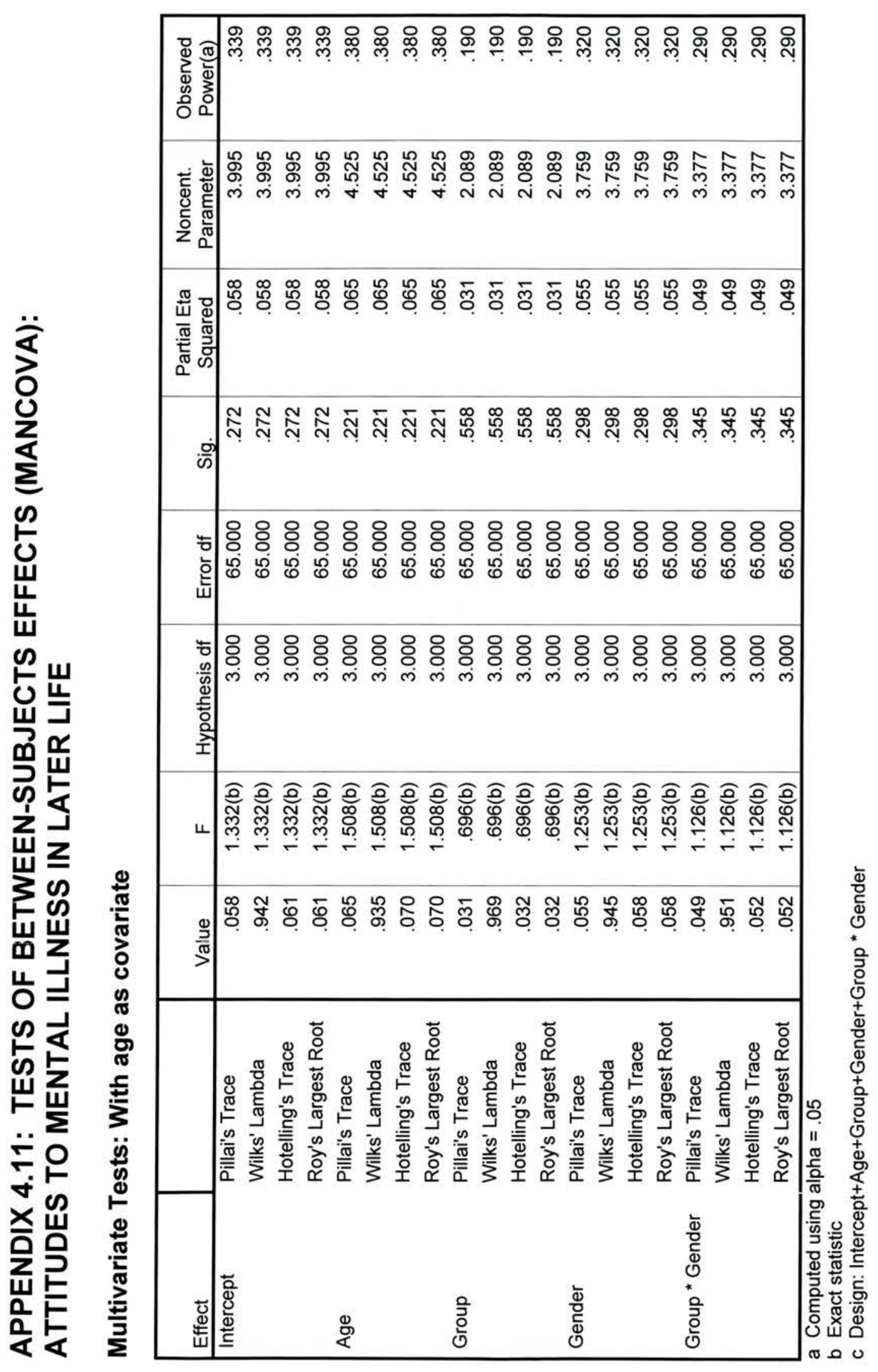




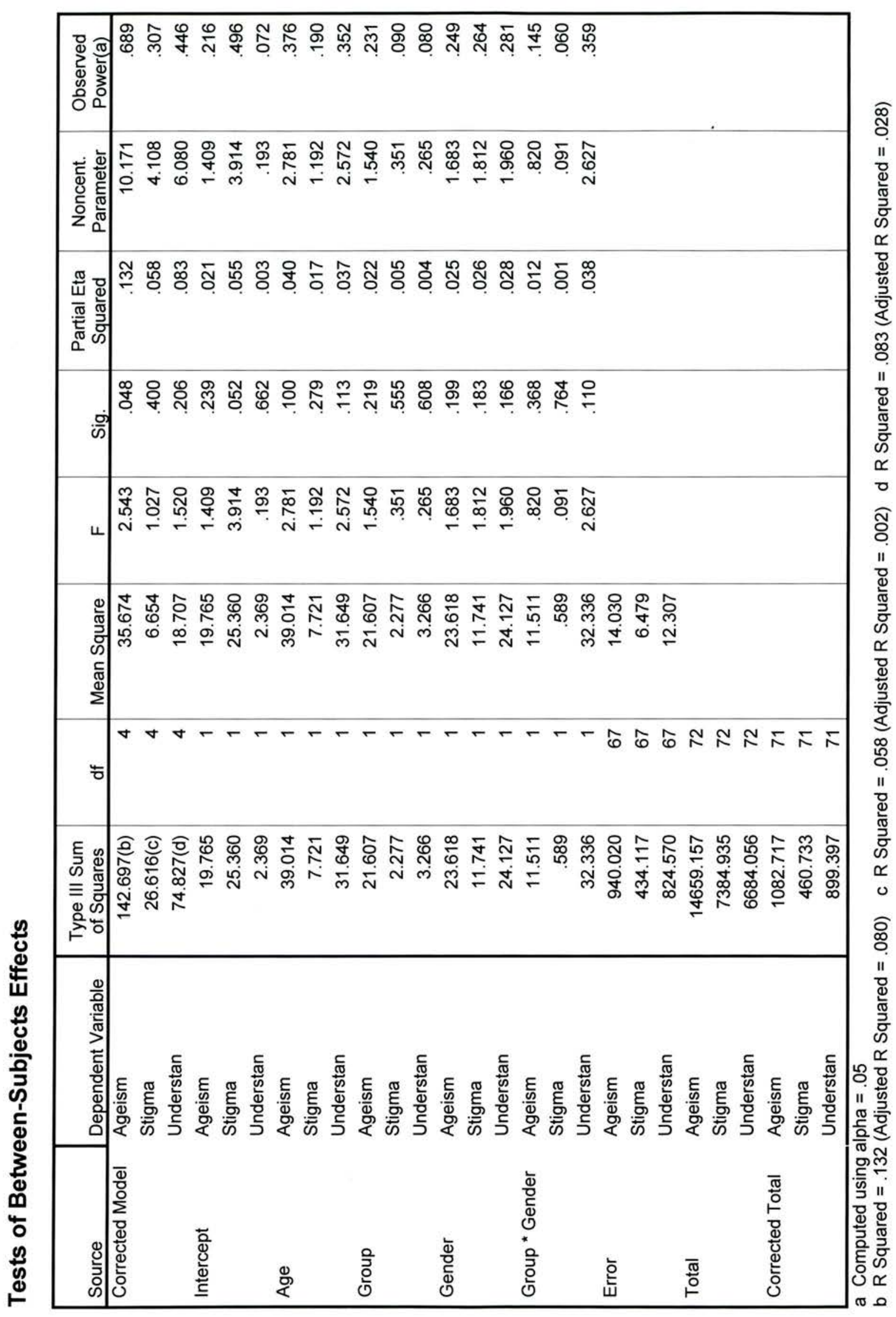




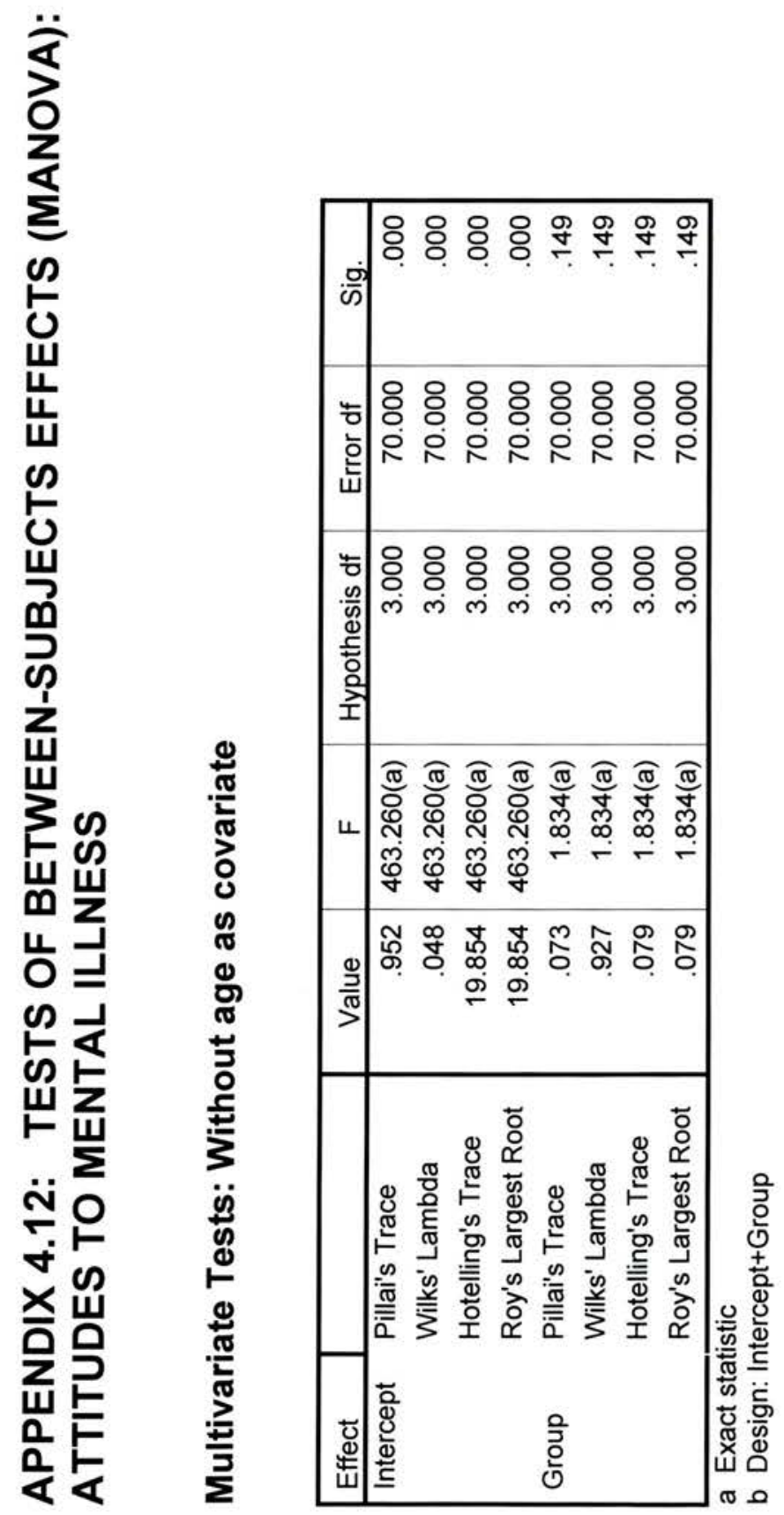




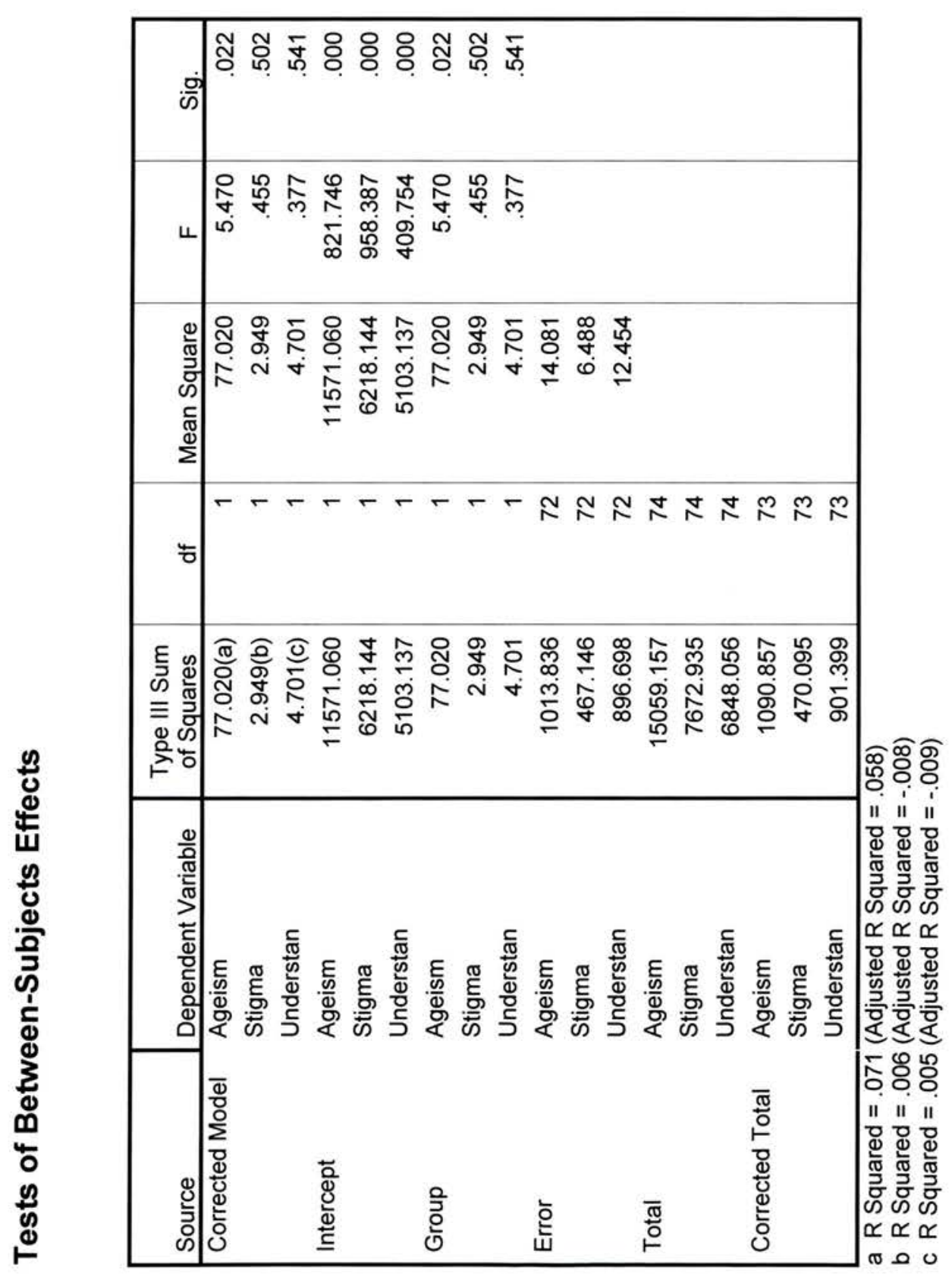




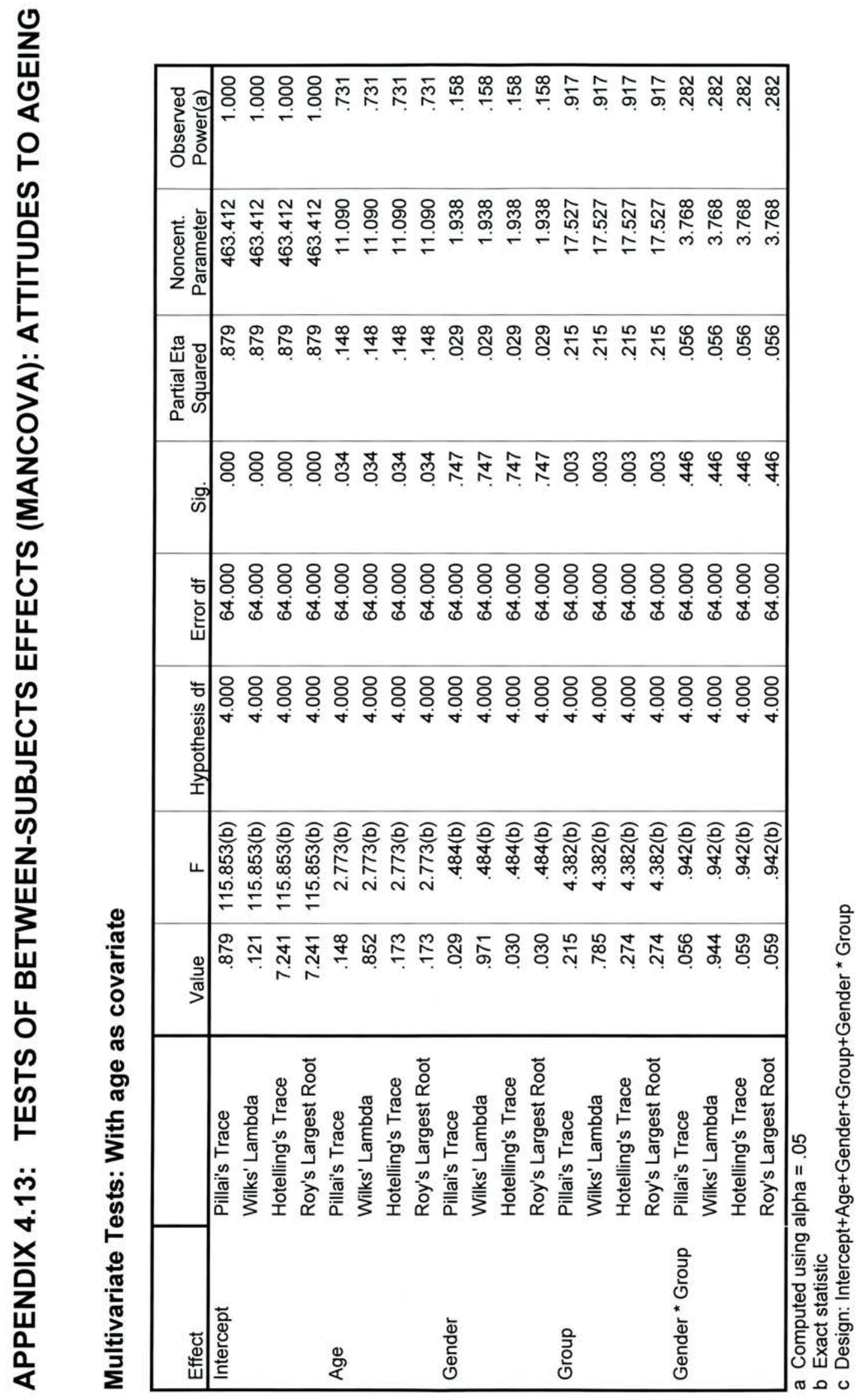




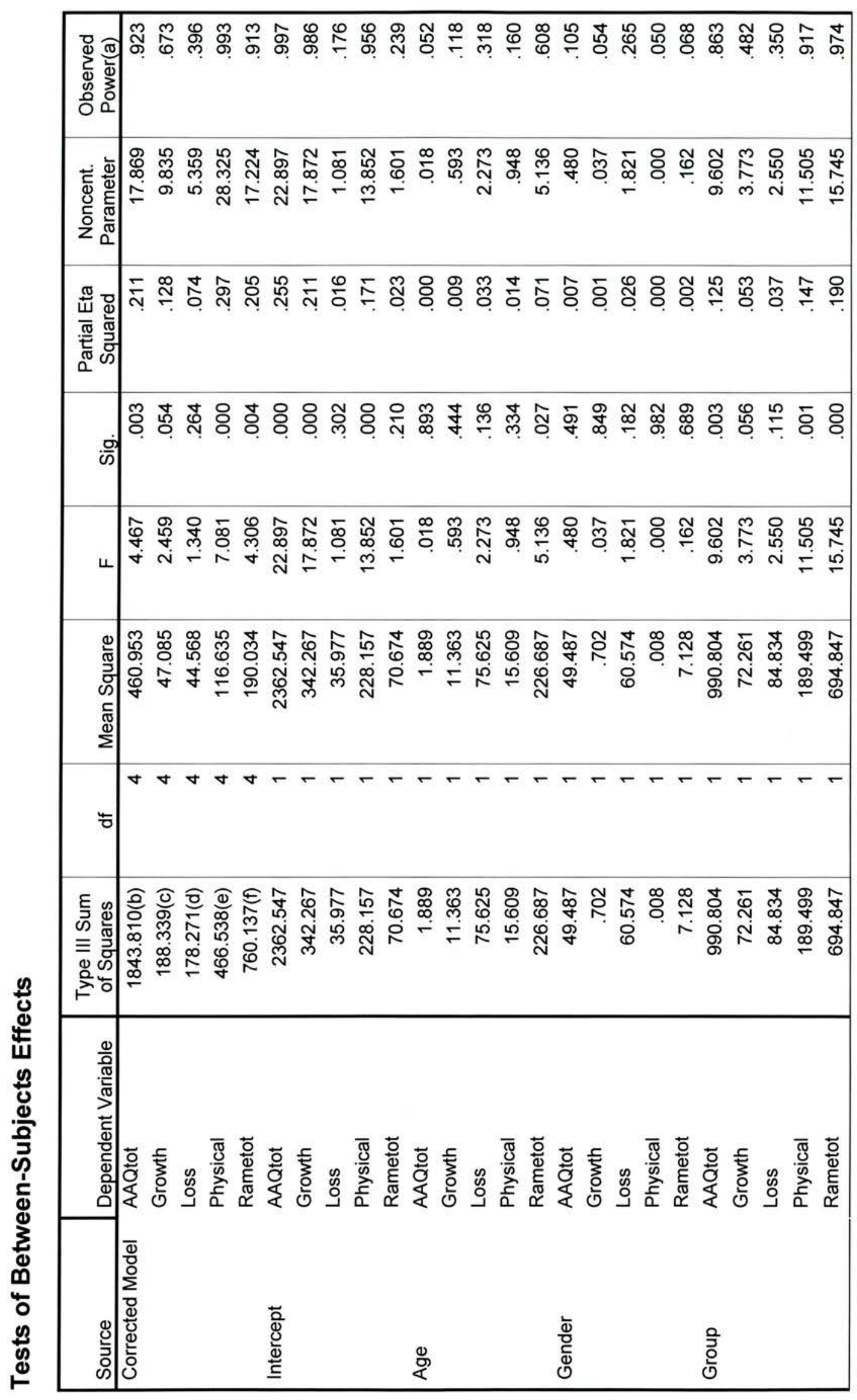




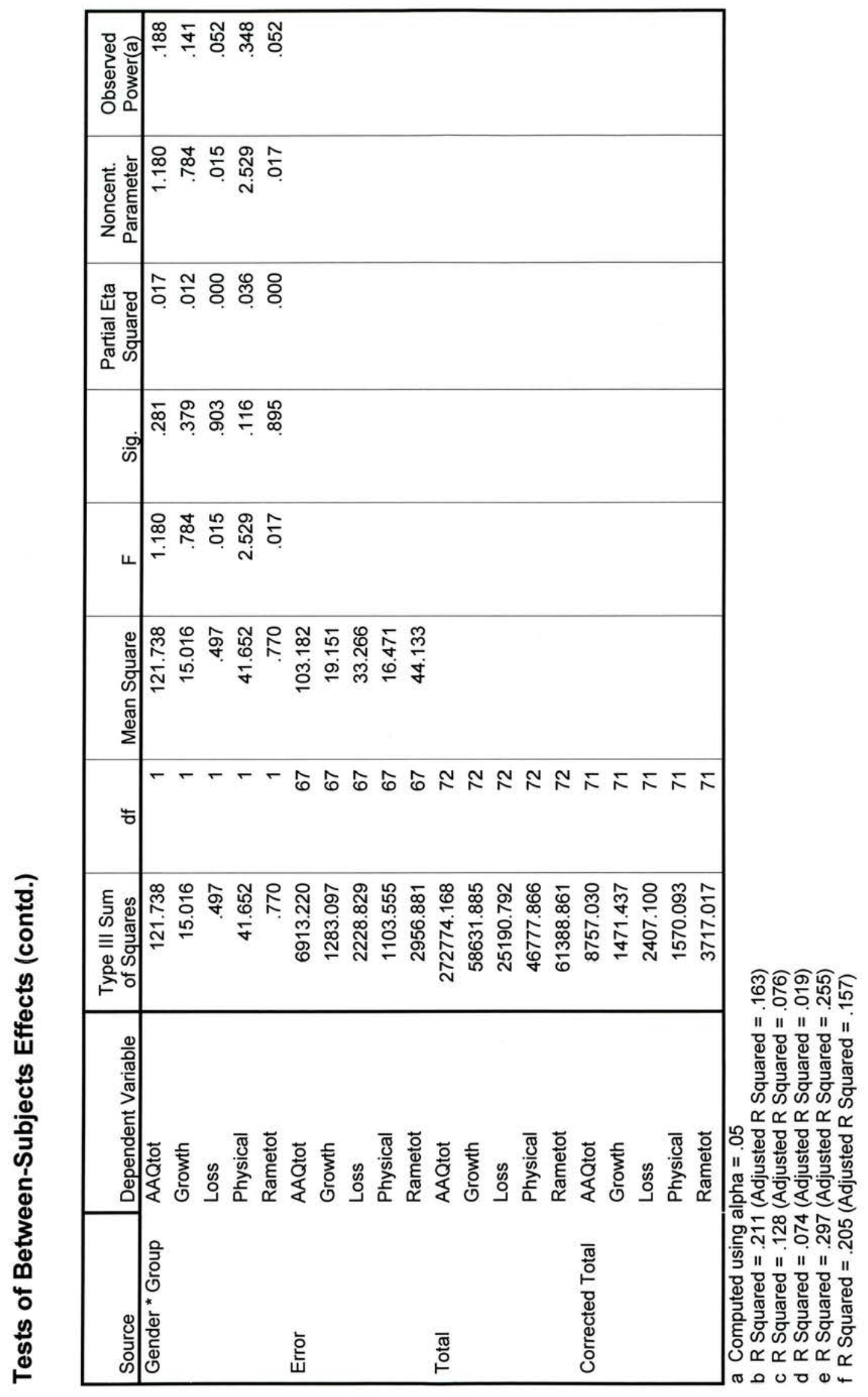




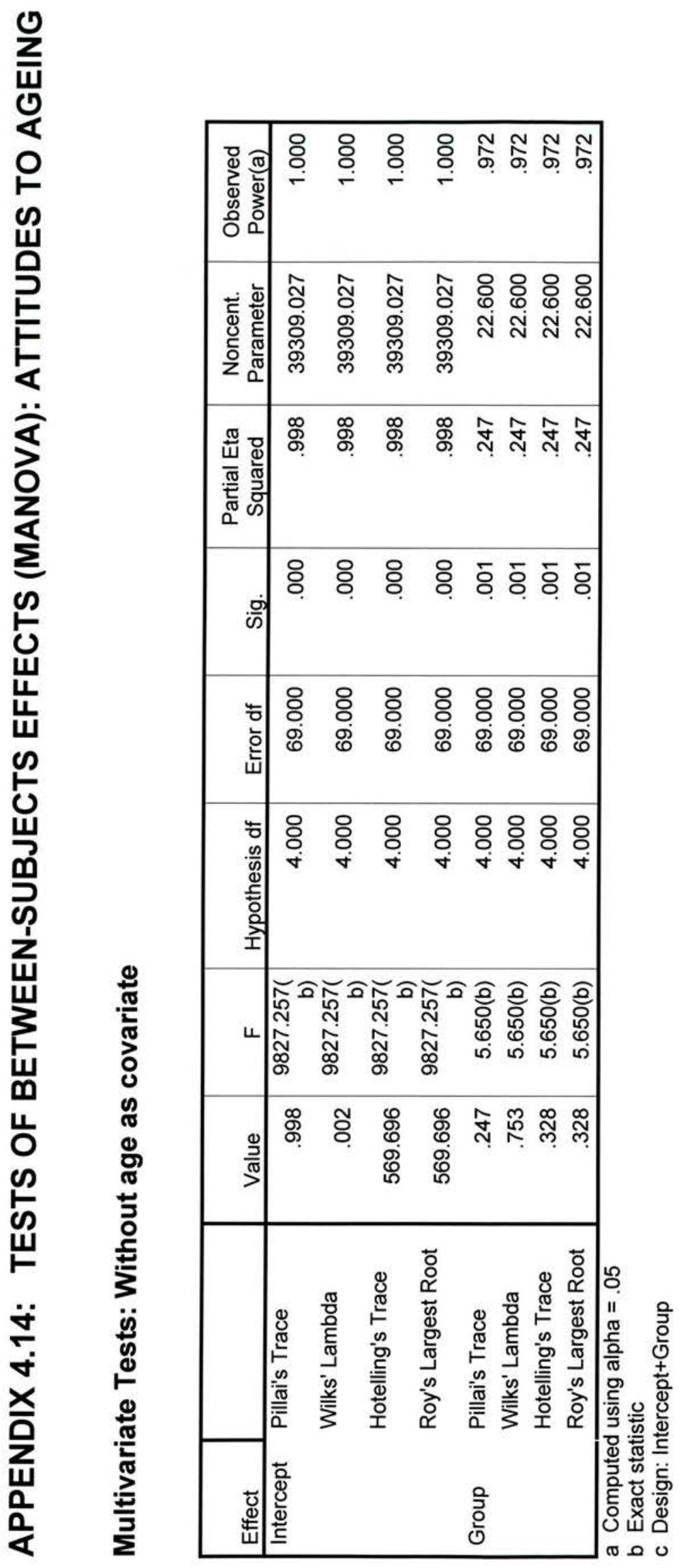




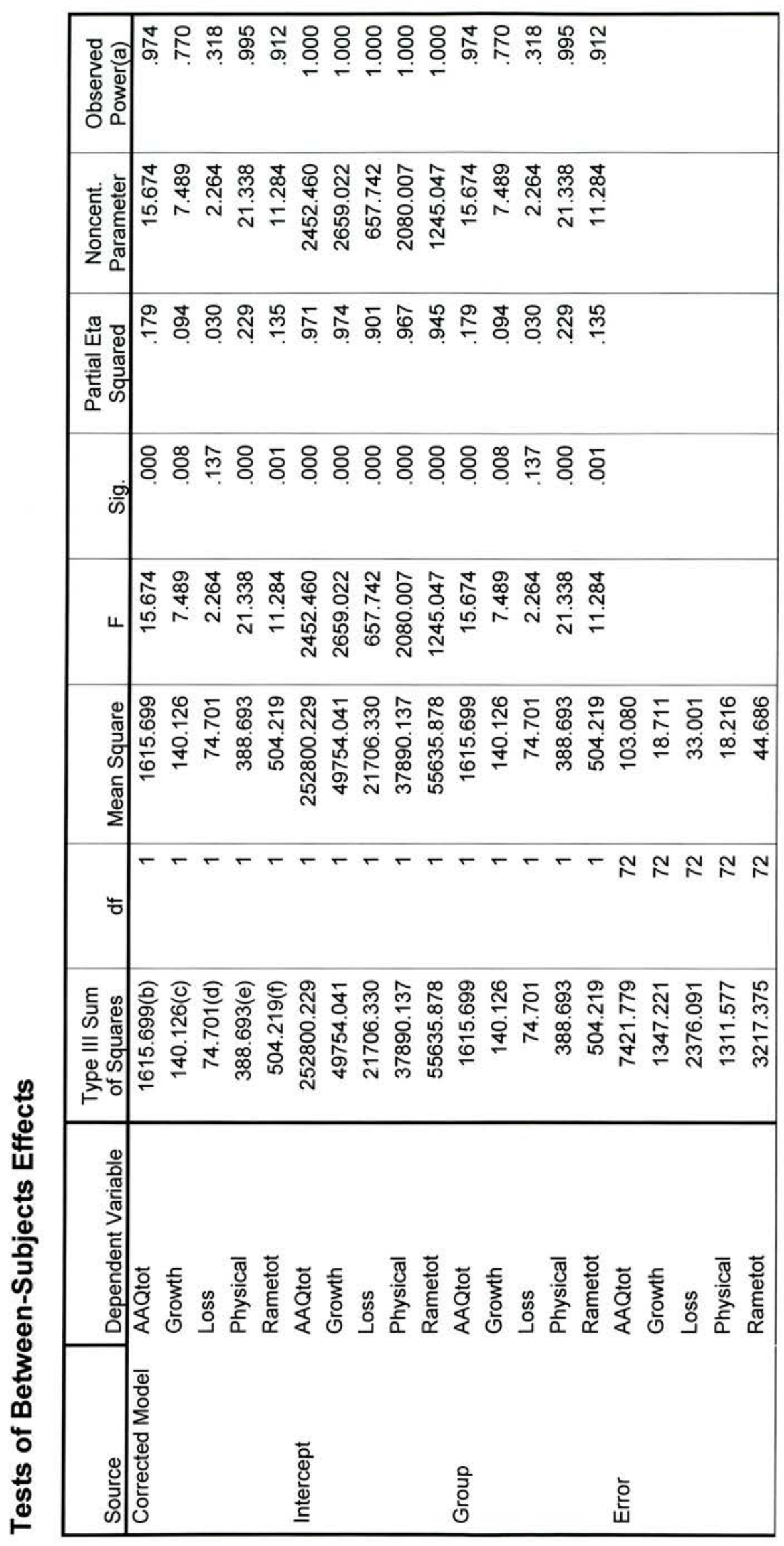




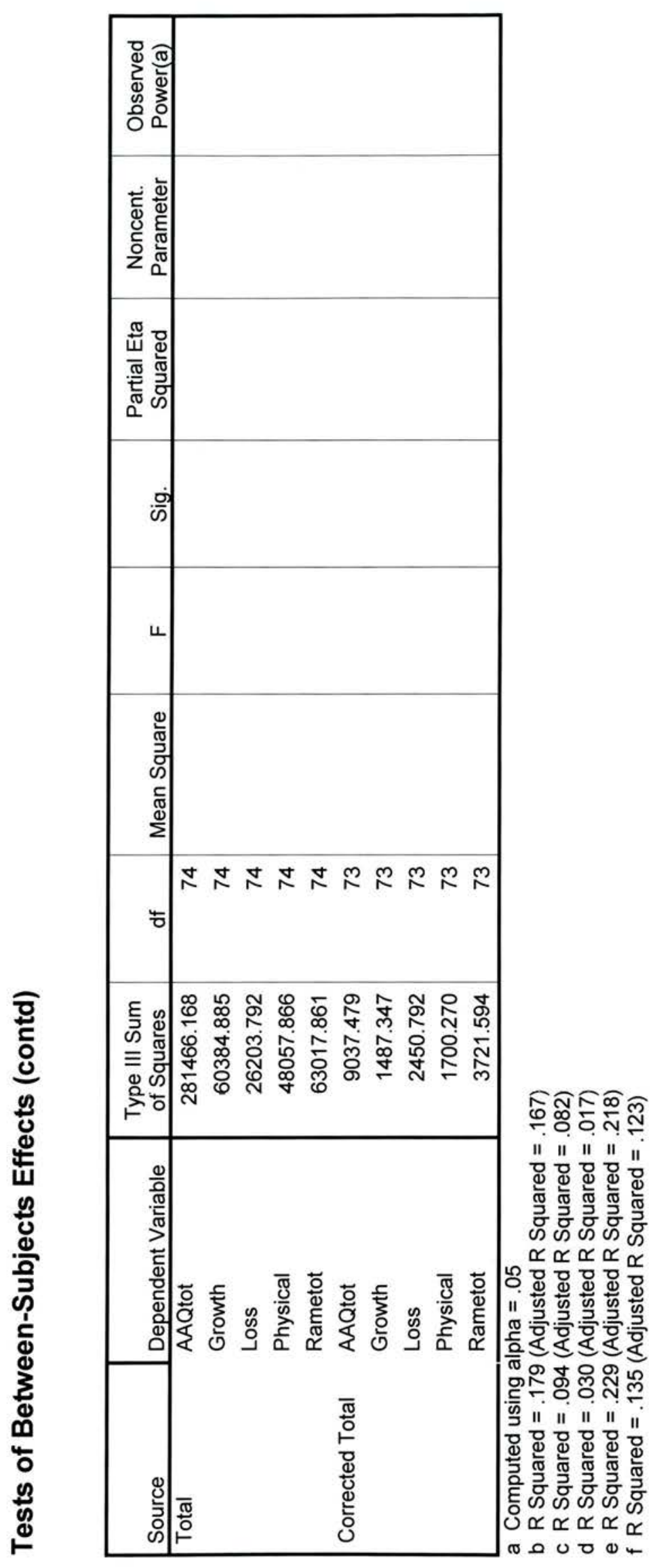


APPENDIX 4.15: BOXPLOTS OF ATTITUDES TO AGEING SCALES BY GROUP

\section{AAQ Composite Score by Group}

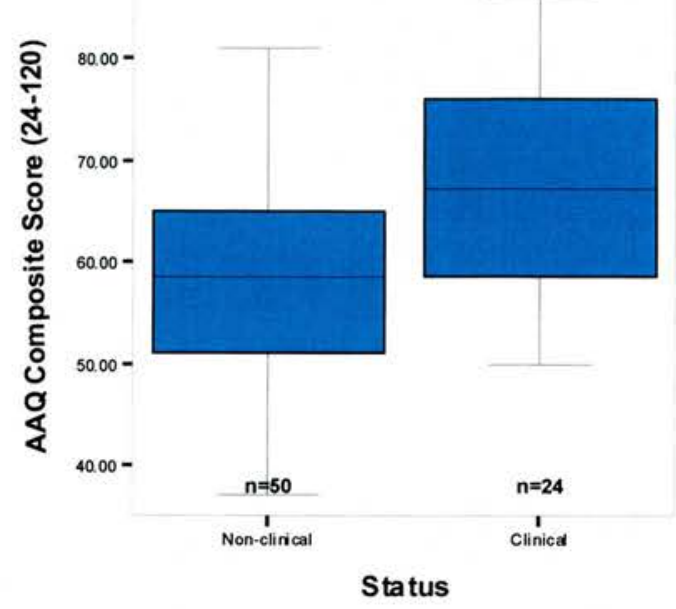

AAQ:Growth Sub-Scale Score by Group

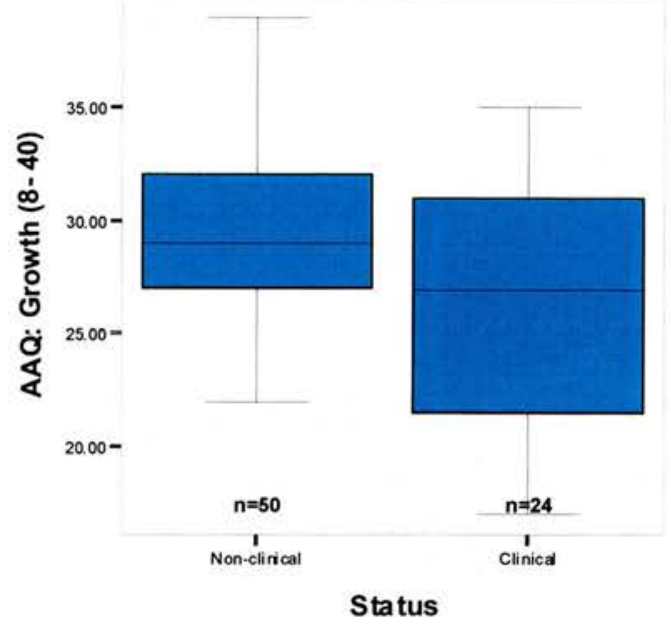




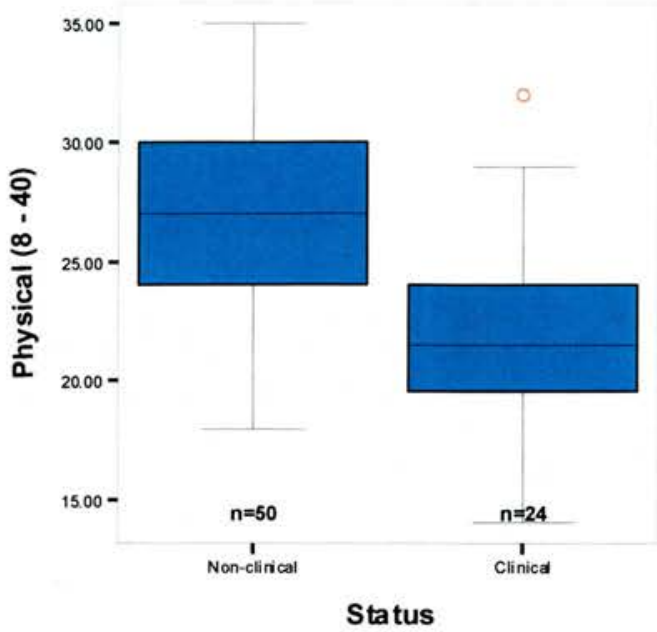

Rame Total Score by Group

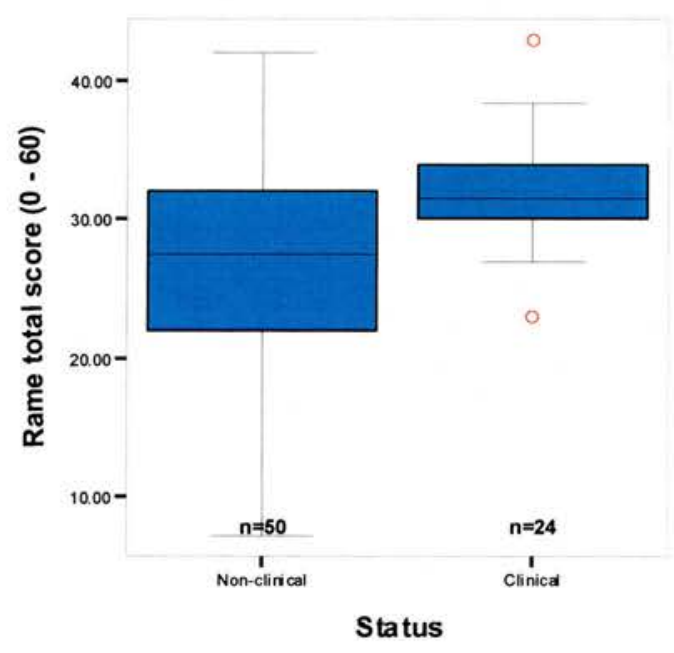




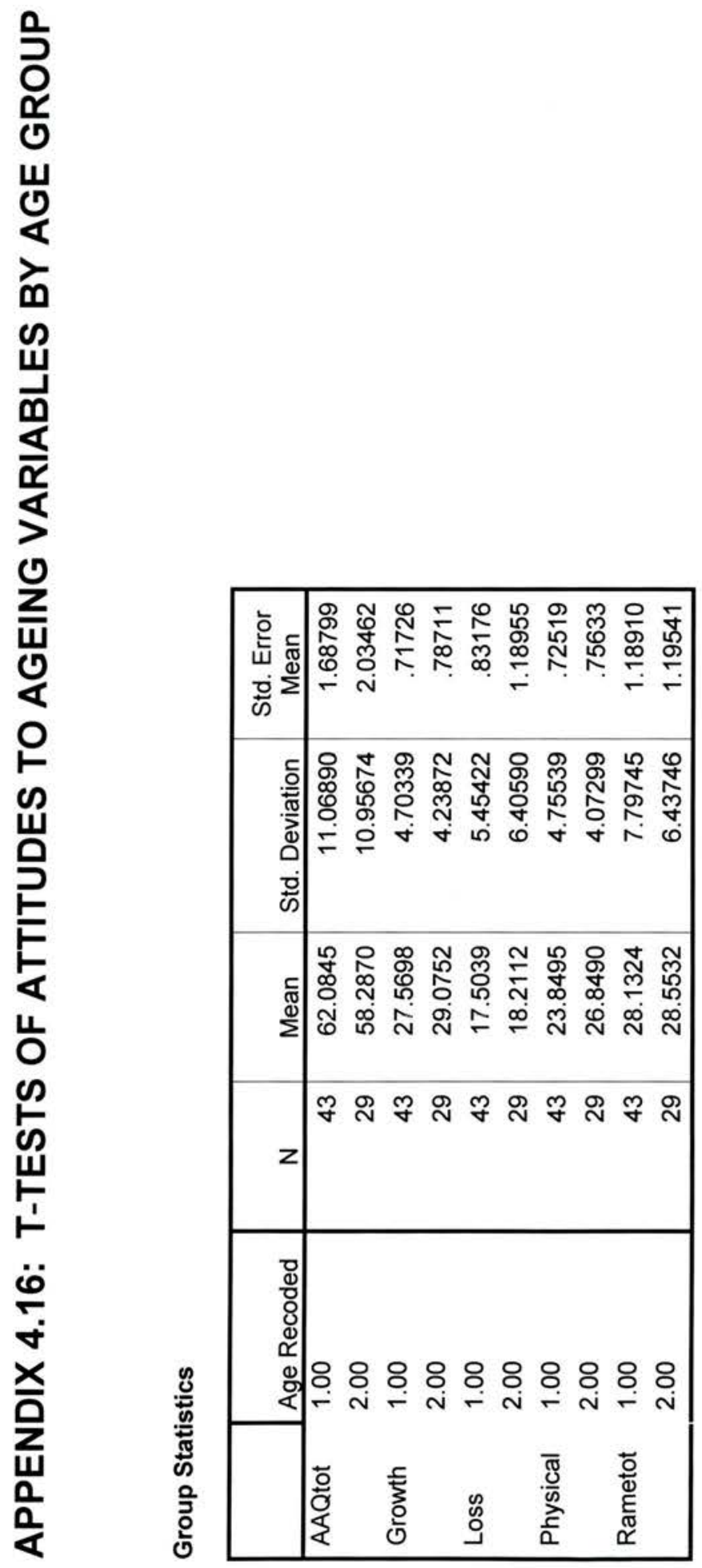




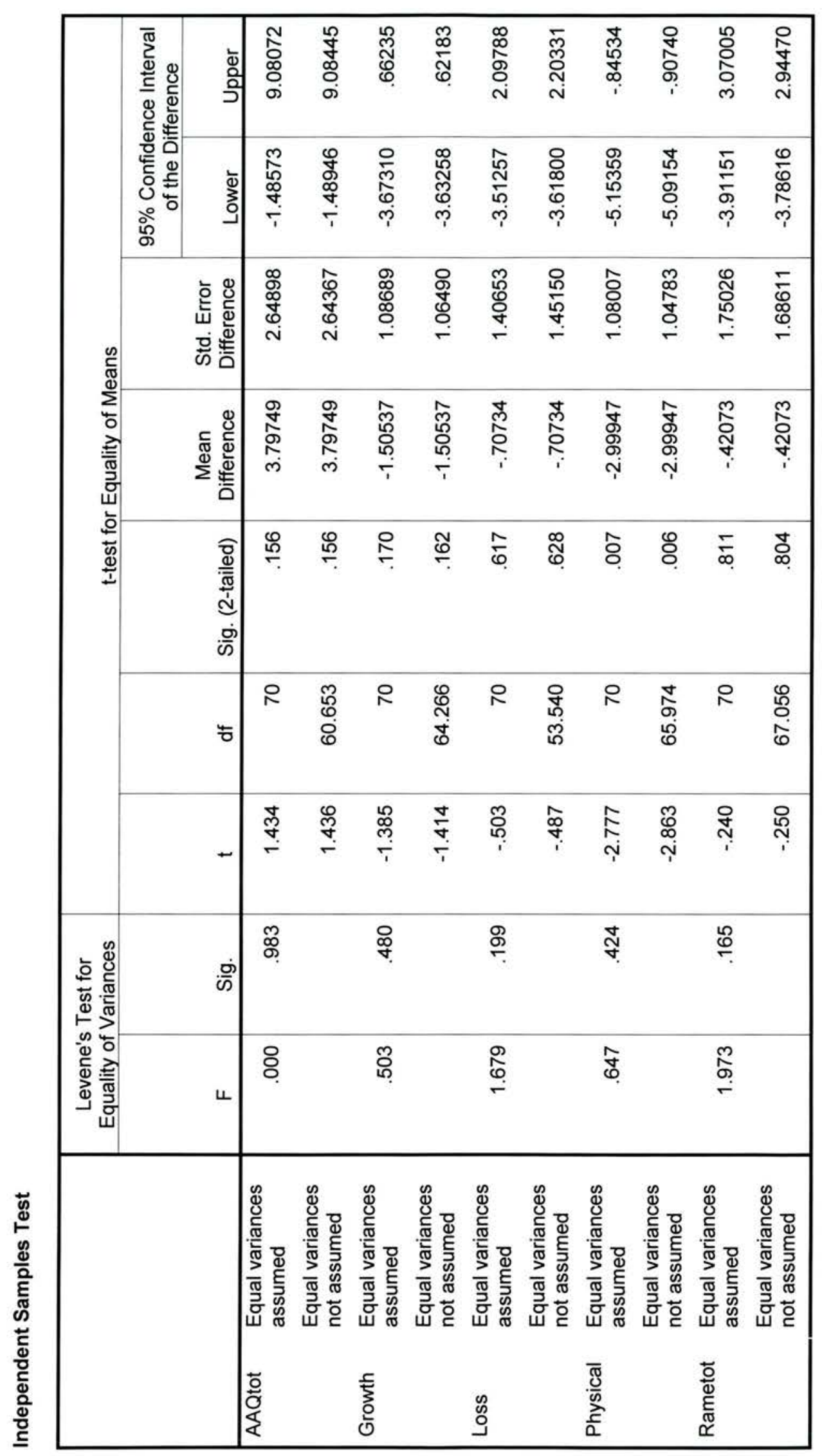


过

产

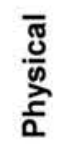

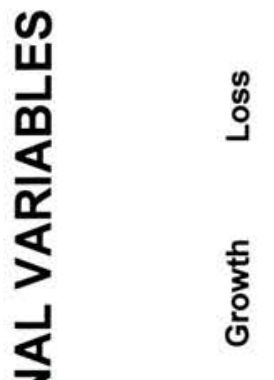

은 总

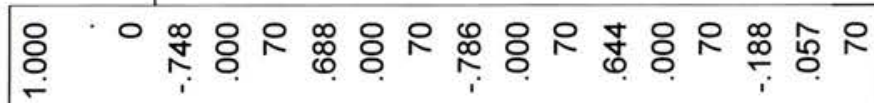

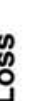

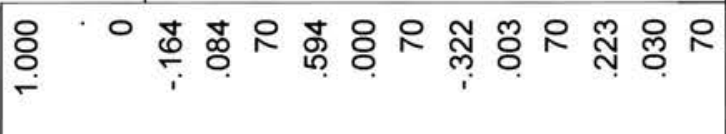

出

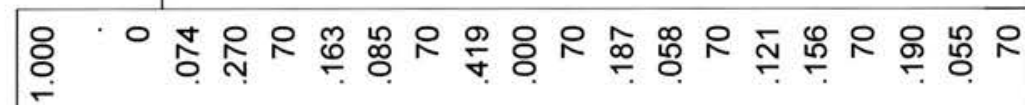

㟧

足

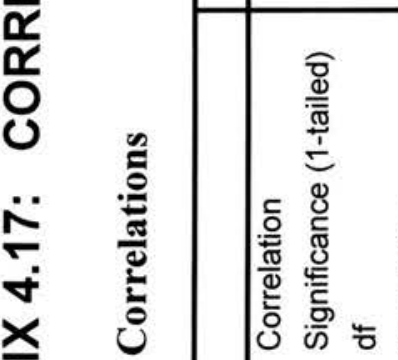

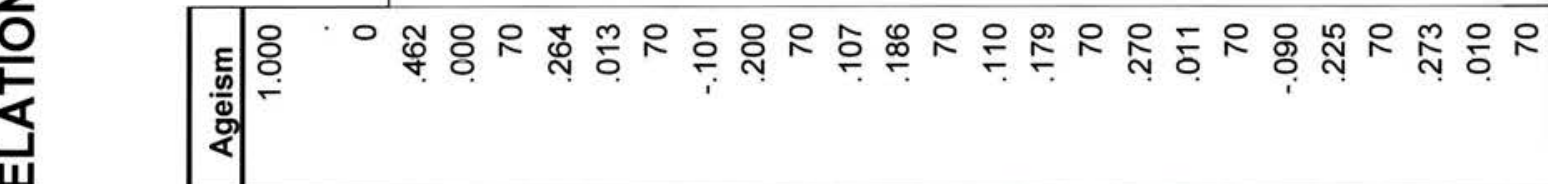

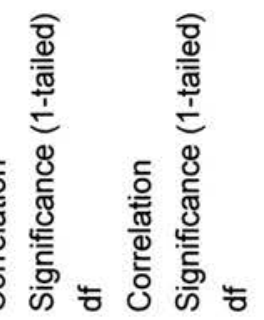

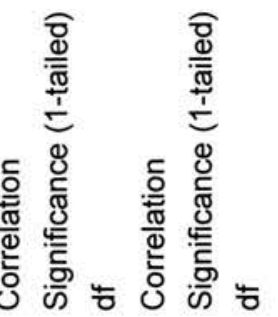

总

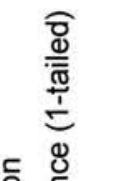

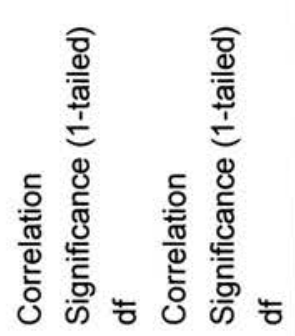

这

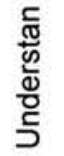

容

亦

号

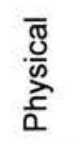

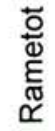

$\stackrel{0}{8}$ 


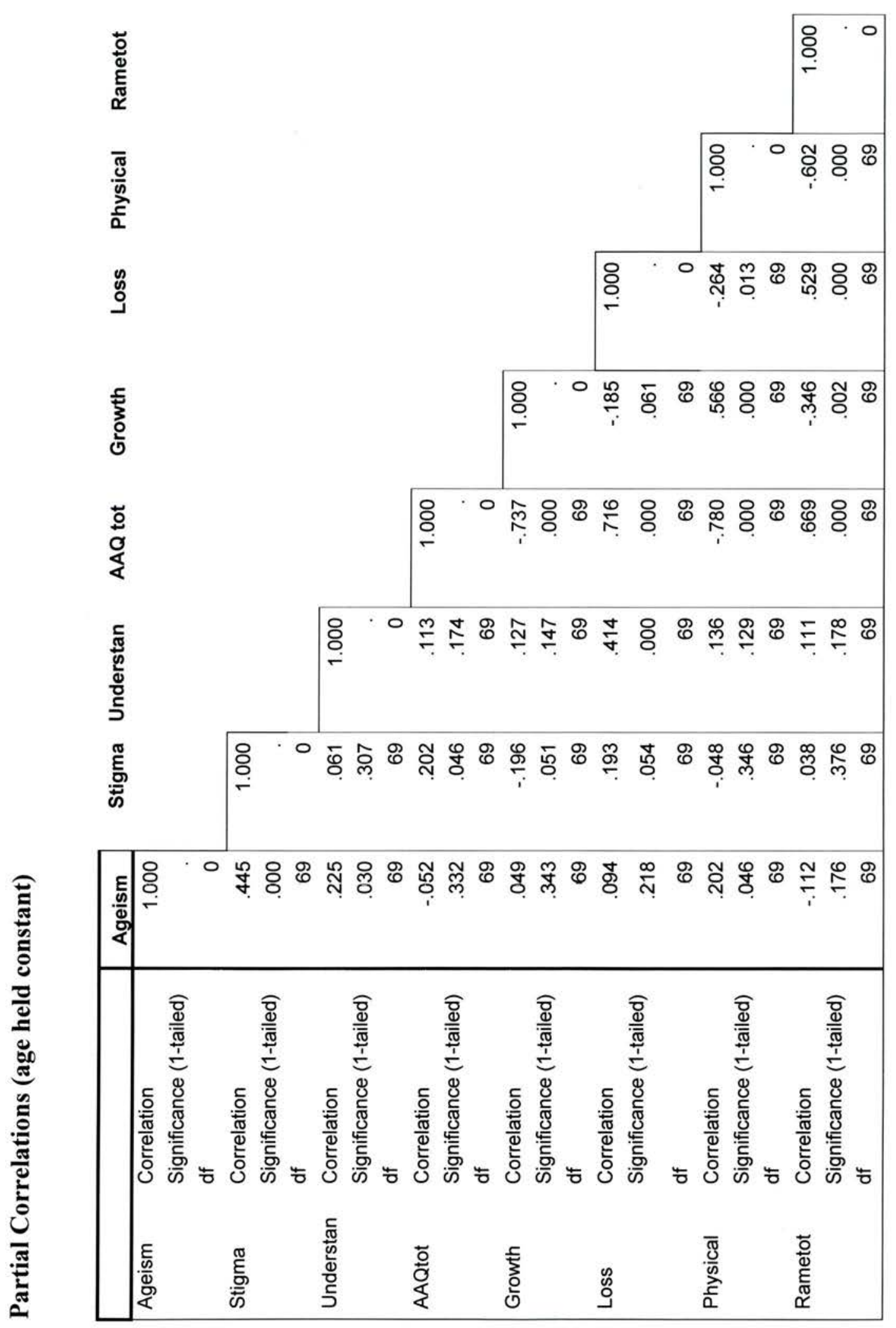




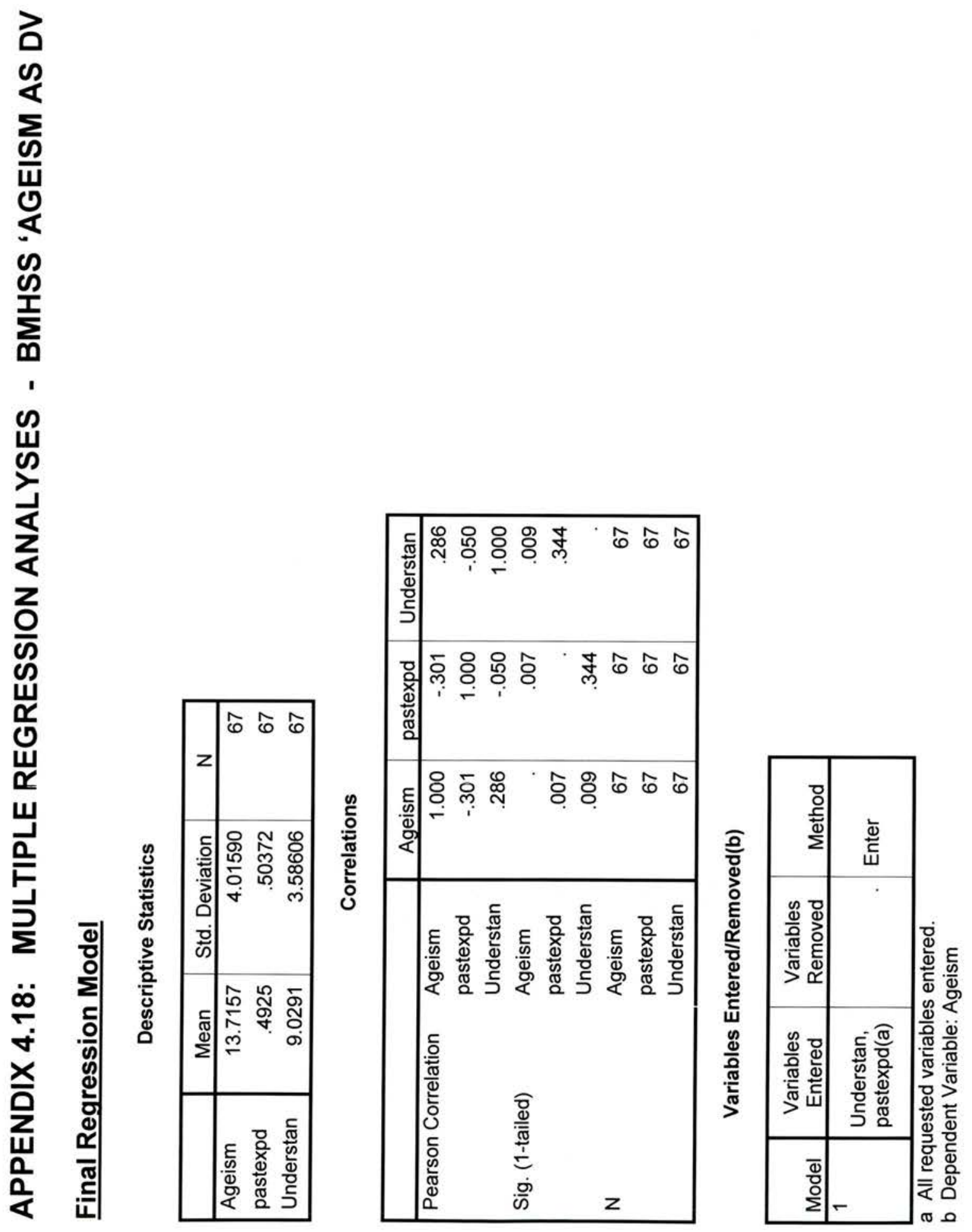




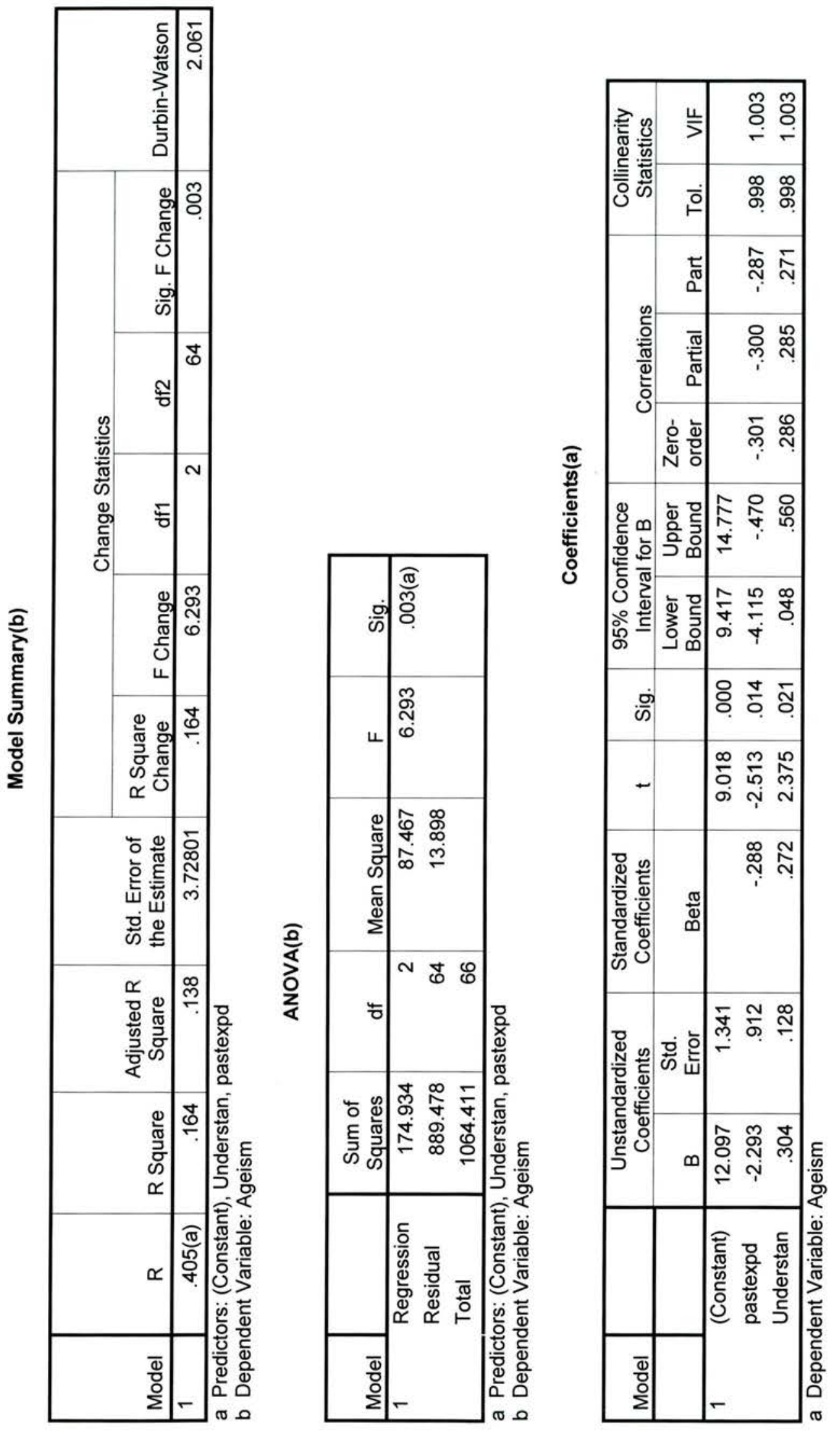




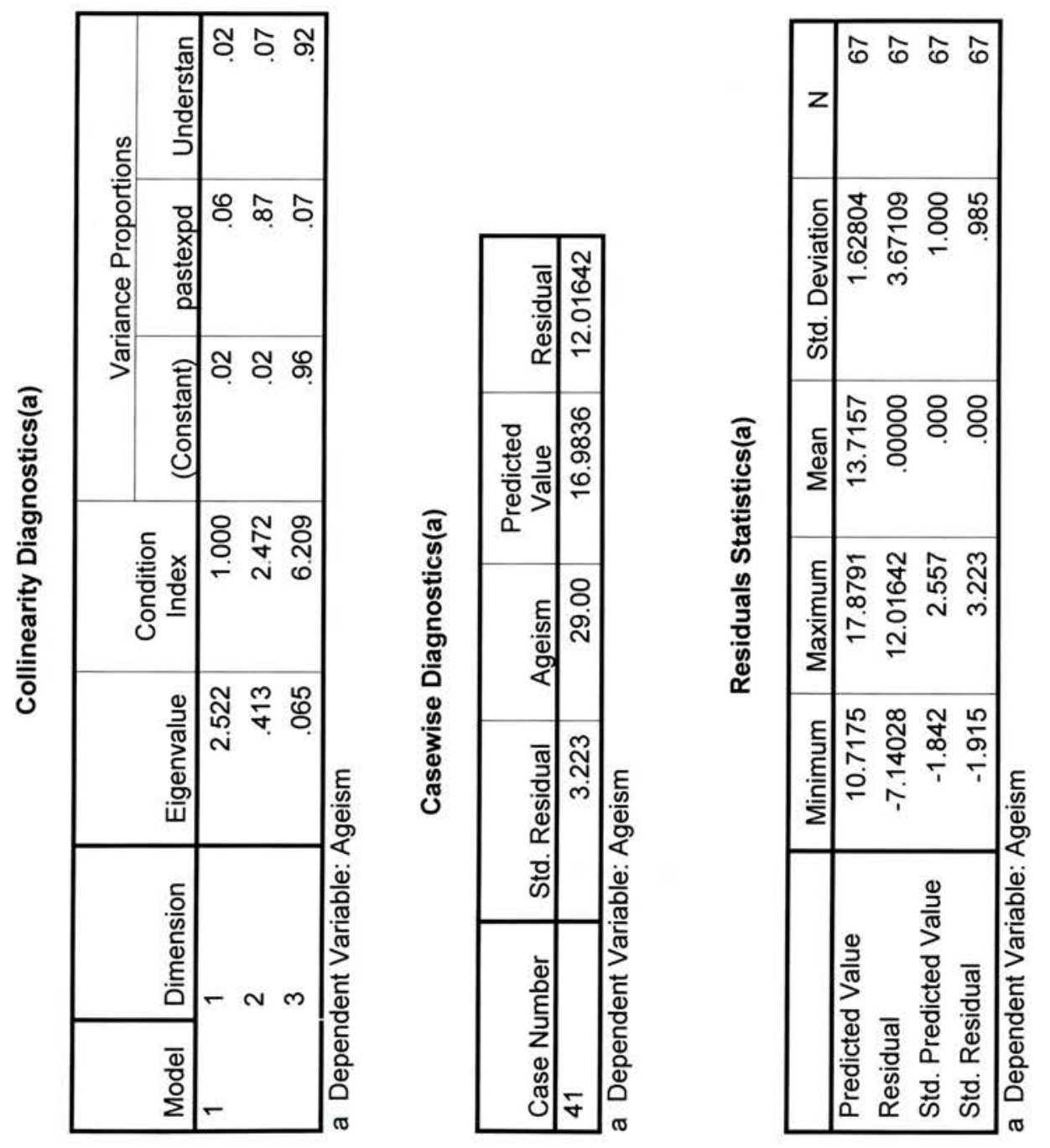




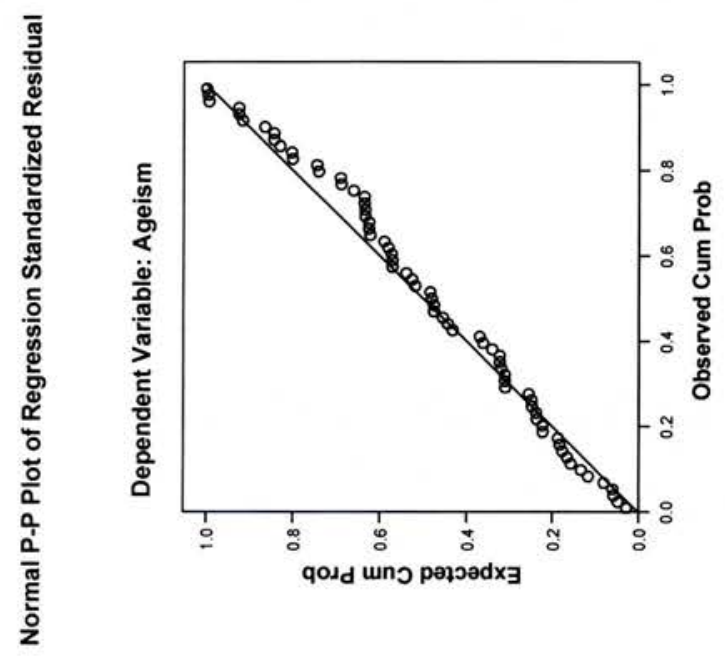

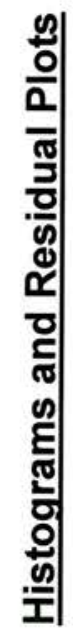

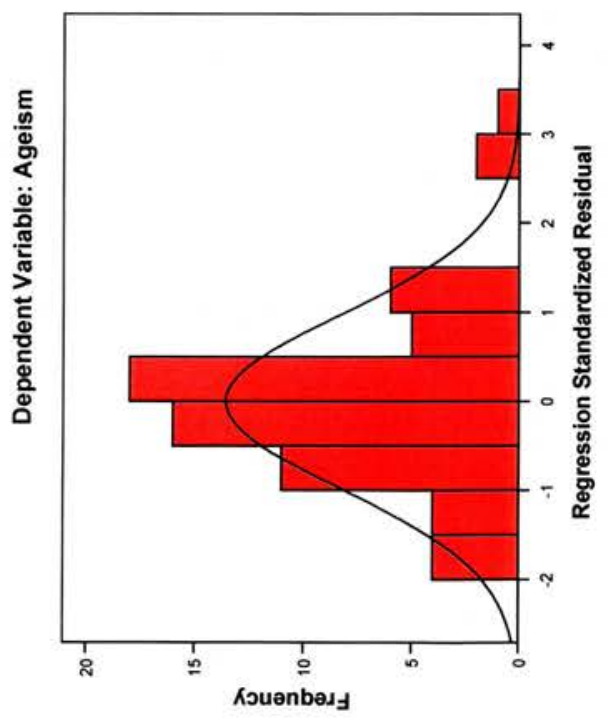




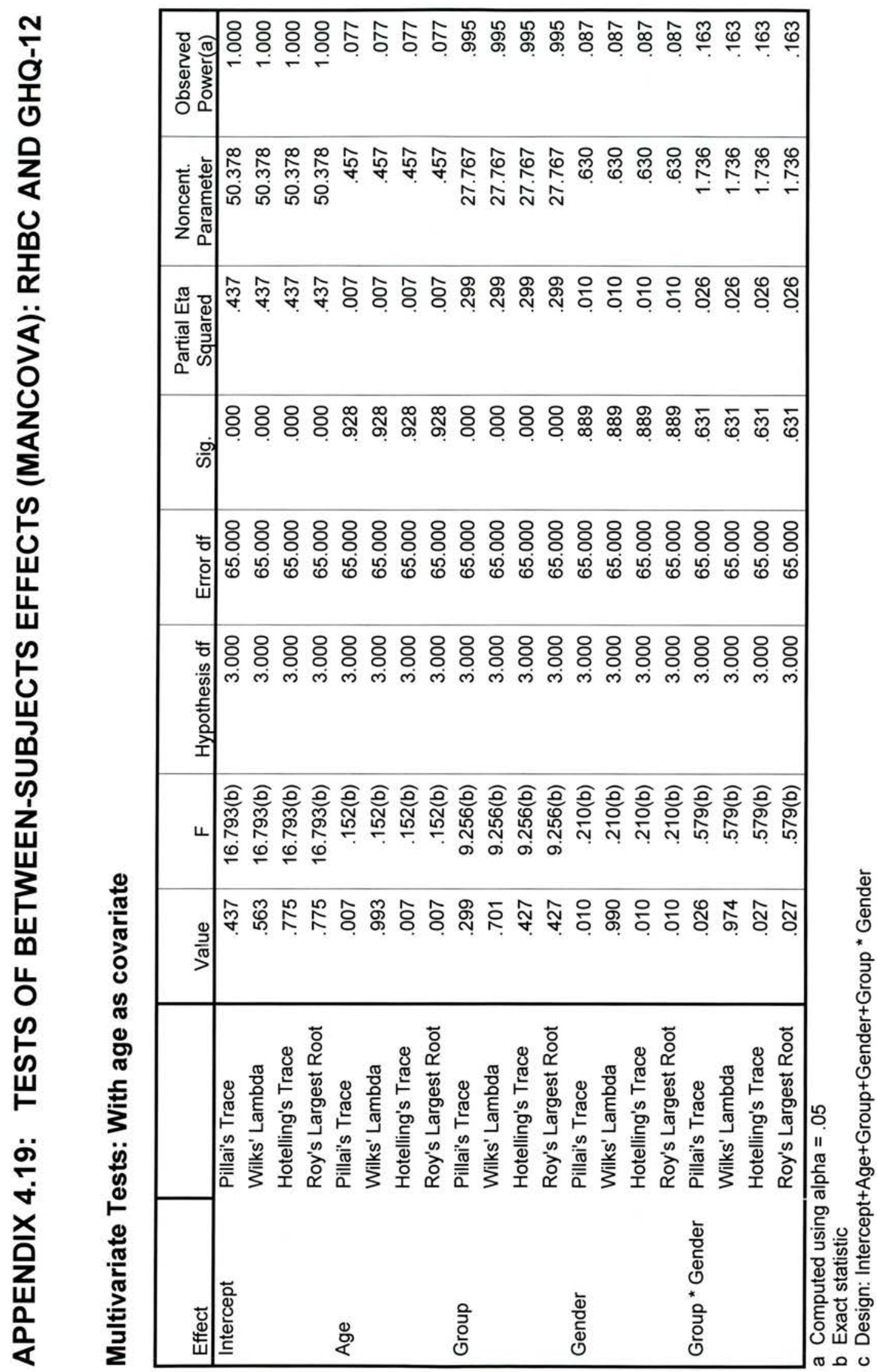




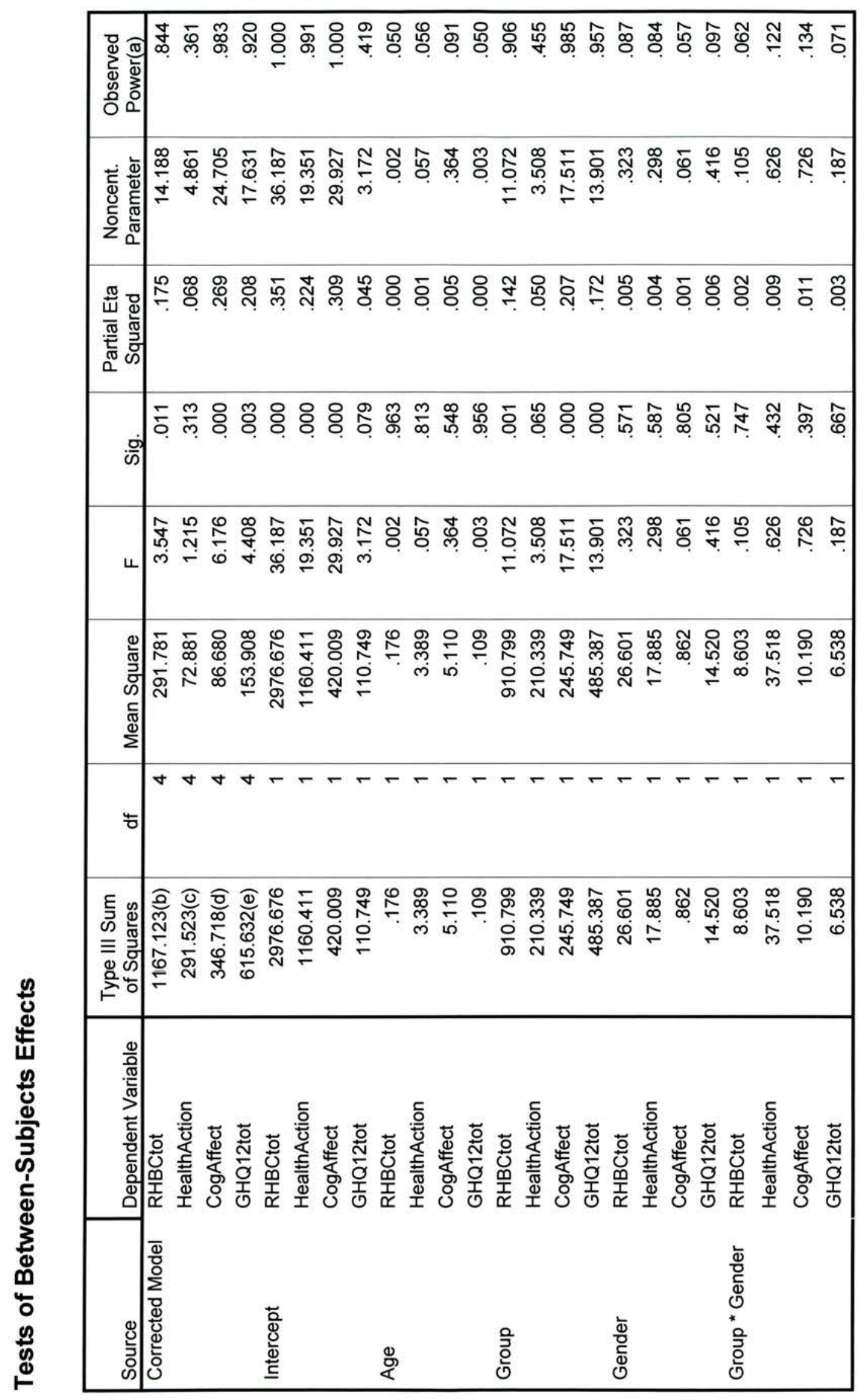




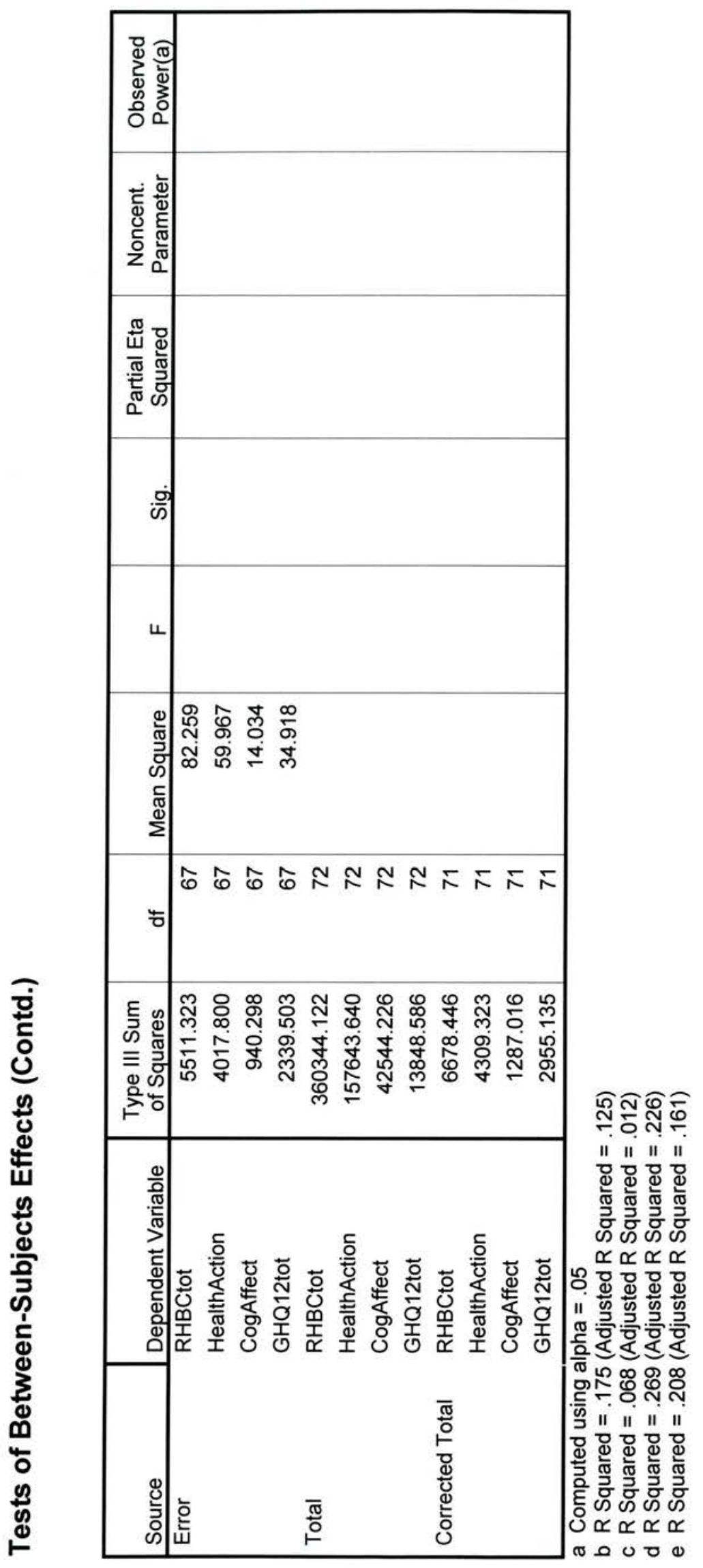




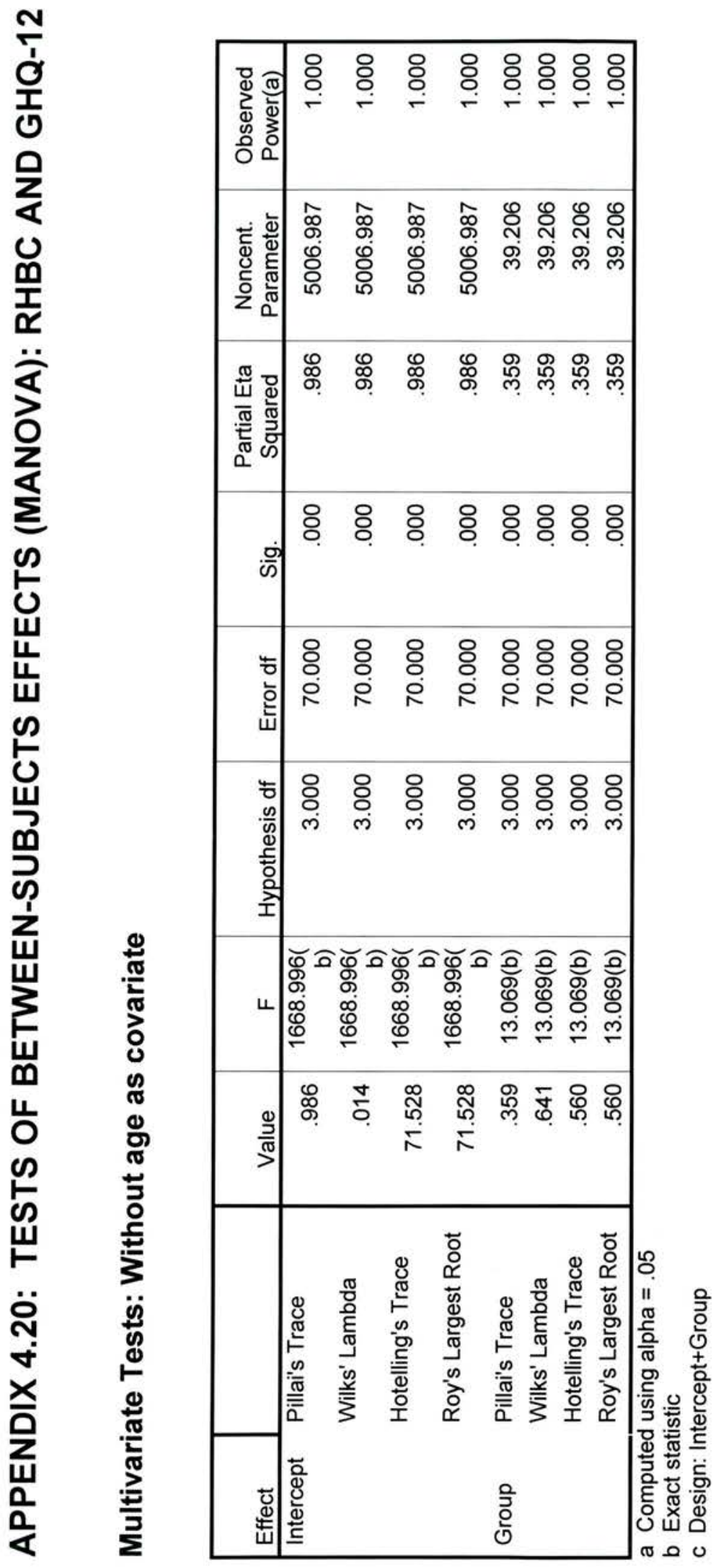




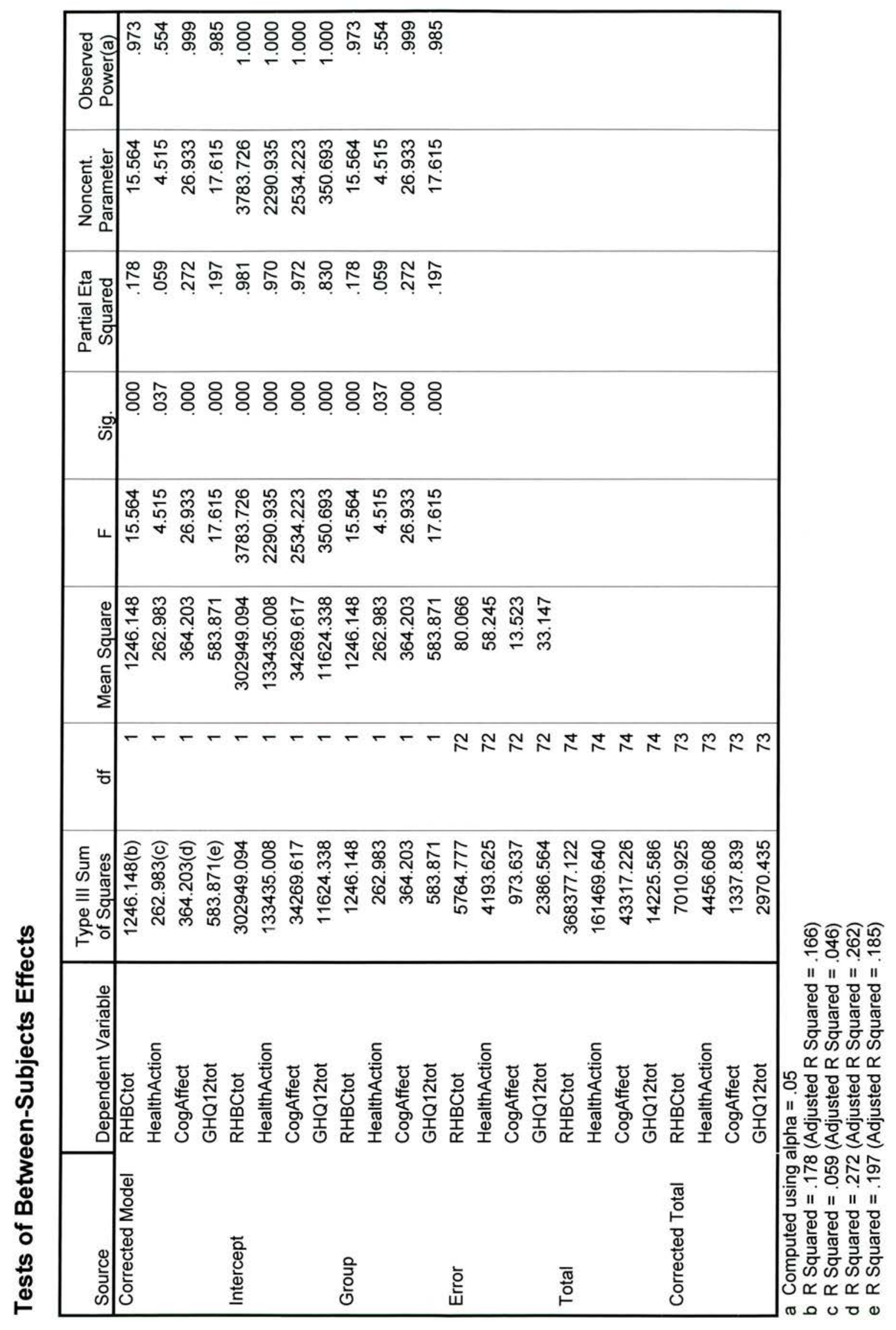




\section{APPENDIX 4.21: BOXPLOTS OF RHBC SCALES AND GHQ-12}

BY GROUP

\section{RHBC Total Score by Group}

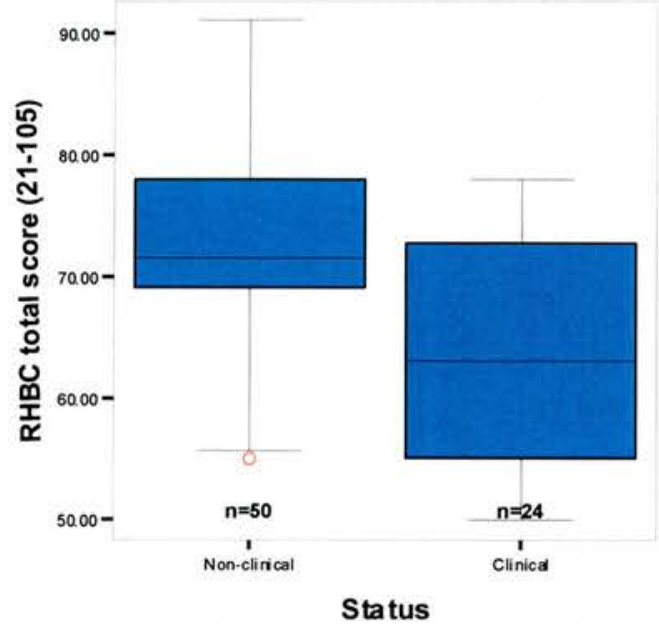

RHBC 'Health Action' Subscale by Group

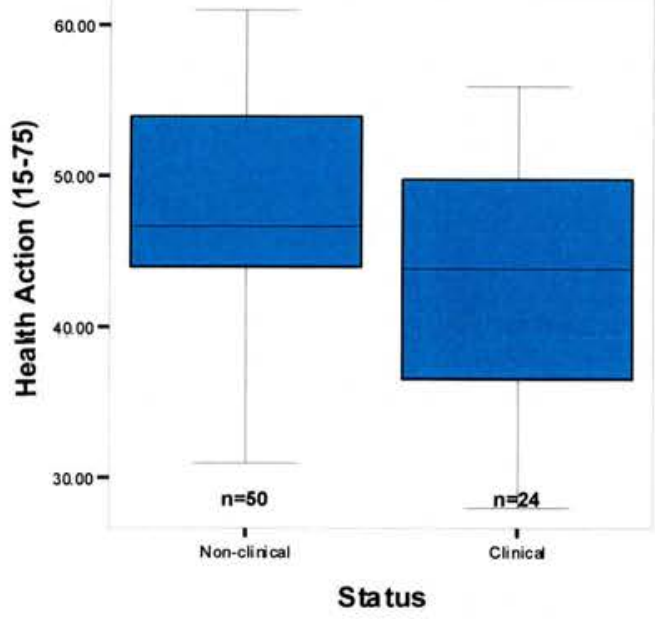




\section{$\underline{\text { RHBC 'Cognitive Affective' Subscale by Group }}$}

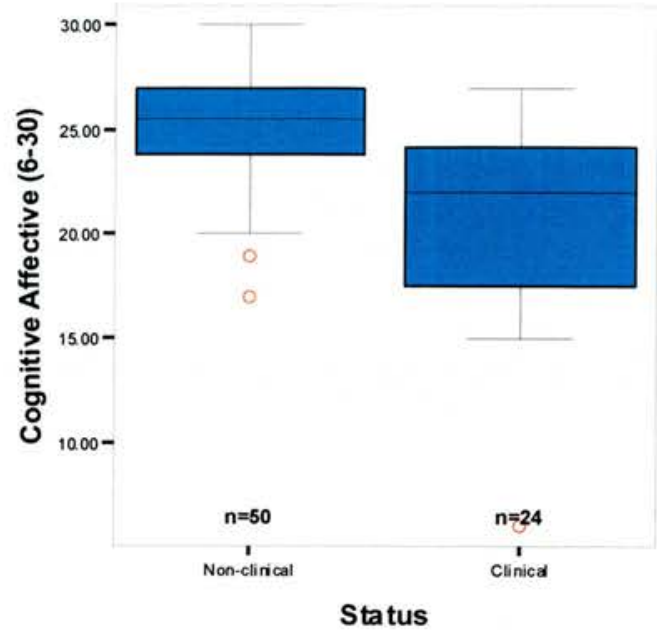

\section{GHQ-12 Score by Group}

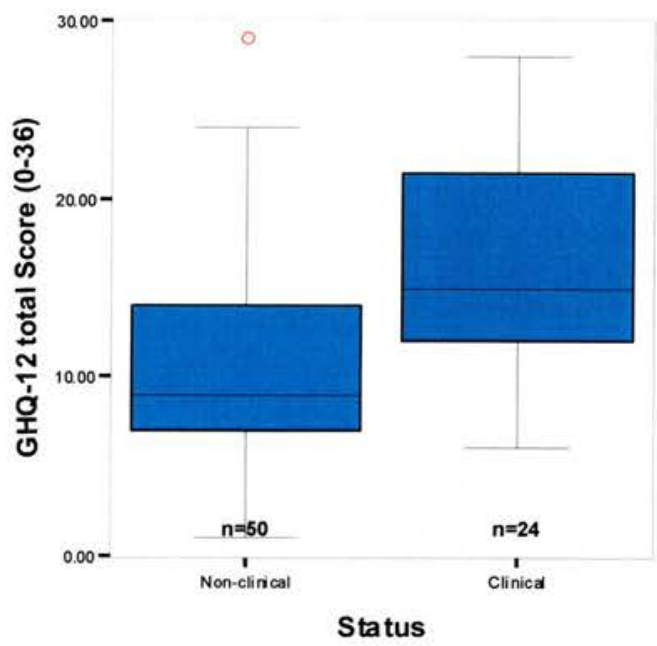




\section{APPENDIX 4.22: CORRELATIONS BETWEEN HEALTH BEHAVIOURS, SUBJECTIVE WELL-BEING AND ATTITUDINAL VARIABLES}

\section{Zero-Order Correlations}

\begin{tabular}{|c|c|c|c|c|c|}
\hline \multirow{2}{*}{ RHBCtot } & & RHBCtot & \multirow[t]{2}{*}{ HealthAction } & \multirow[t]{3}{*}{ CogAffect } & \multirow[t]{2}{*}{ GHQ12tot } \\
\hline & $\begin{array}{l}\text { Correlation } \\
\text { Significance (1-tailed) } \\
\text { df }\end{array}$ & $\begin{array}{r}1.000 \\
0\end{array}$ & & & \\
\hline HealthAction & $\begin{array}{l}\text { Correlation } \\
\text { Significance (1-tailed) } \\
\text { df }\end{array}$ & $\begin{array}{r}.904 \\
.000 \\
70\end{array}$ & $\begin{array}{r}1.000 \\
0\end{array}$ & & \\
\hline CogAffect & $\begin{array}{l}\text { Correlation } \\
\text { Significance (1-tailed) } \\
\text { df }\end{array}$ & $\begin{array}{r}.624 \\
.000 \\
70\end{array}$ & $\begin{array}{r}.230 \\
.026 \\
70\end{array}$ & $\begin{array}{r}1.000 \\
0\end{array}$ & \\
\hline GHQ12tot & $\begin{array}{l}\text { Correlation } \\
\text { Significance (1-tailed) } \\
\text { df }\end{array}$ & $\begin{array}{r}-.318 \\
.003 \\
70\end{array}$ & $\begin{array}{r}-.216 \\
.034 \\
70\end{array}$ & $\begin{array}{r}-.330 \\
.002 \\
70\end{array}$ & 1.000 \\
\hline Ageism & $\begin{array}{l}\text { Correlation } \\
\text { Significance (1-tailed) } \\
\text { df }\end{array}$ & $\begin{array}{r}-.013 \\
.457 \\
70\end{array}$ & $\begin{array}{r}-.080 \\
.251 \\
70\end{array}$ & $\begin{array}{r}.118 \\
.163 \\
70\end{array}$ & $\begin{array}{r}-.161 \\
.088 \\
70\end{array}$ \\
\hline Stigma & $\begin{array}{l}\text { Correlation } \\
\text { Significance (1-tailed) } \\
\text { df }\end{array}$ & $\begin{array}{r}-.050 \\
.339 \\
70\end{array}$ & $\begin{array}{r}-.025 \\
.418 \\
70\end{array}$ & $\begin{array}{r}-.068 \\
.285 \\
70\end{array}$ & $\begin{array}{r}-.130 \\
.139 \\
70\end{array}$ \\
\hline Understan & $\begin{array}{l}\text { Correlation } \\
\text { Significance (1-tailed) } \\
\text { df }\end{array}$ & $\begin{array}{r}.122 \\
.154 \\
70\end{array}$ & $\begin{array}{r}.152 \\
.101 \\
70\end{array}$ & $\begin{array}{r}-.001 \\
.495 \\
70\end{array}$ & $\begin{array}{r}.132 \\
.134 \\
70\end{array}$ \\
\hline AAQtot & $\begin{array}{l}\text { Correlation } \\
\text { Significance (1-tailed) } \\
\text { df }\end{array}$ & $\begin{array}{r}-.390 \\
.000 \\
70\end{array}$ & $\begin{array}{r}-.206 \\
.041 \\
70\end{array}$ & $\begin{array}{r}-.512 \\
.000 \\
70\end{array}$ & $\begin{array}{r}.622 \\
.000 \\
70\end{array}$ \\
\hline Growth & $\begin{array}{l}\text { Correlation } \\
\text { Significance (1-tailed) } \\
\text { df }\end{array}$ & $\begin{array}{r}.420 \\
.000 \\
70\end{array}$ & $\begin{array}{r}.282 \\
.008 \\
70\end{array}$ & $\begin{array}{r}.441 \\
.000 \\
70\end{array}$ & $\begin{array}{r}-.332 \\
.002 \\
70\end{array}$ \\
\hline Loss & $\begin{array}{l}\text { Correlation } \\
\text { Significance (1-tailed) } \\
\text { df }\end{array}$ & $\begin{array}{r}-.104 \\
.193 \\
70\end{array}$ & $\begin{array}{r}.036 \\
.384 \\
70\end{array}$ & $\begin{array}{r}-.302 \\
.005 \\
70\end{array}$ & $\begin{array}{r}.539 \\
.000 \\
70\end{array}$ \\
\hline Physical & $\begin{array}{l}\text { Correlation } \\
\text { Significance (1-tailed) } \\
\text { df }\end{array}$ & $\begin{array}{r}.387 \\
.000 \\
70\end{array}$ & $\begin{array}{r}.258 \\
.014 \\
70\end{array}$ & $\begin{array}{r}.408 \\
.000 \\
70\end{array}$ & $\begin{array}{r}-.480 \\
.000 \\
70\end{array}$ \\
\hline Rametot & $\begin{array}{l}\text { Correlation } \\
\text { Significance (1-tailed) } \\
\text { df }\end{array}$ & $\begin{array}{r}-.272 \\
.010 \\
70\end{array}$ & $\begin{array}{r}-.154 \\
.098 \\
70\end{array}$ & $\begin{array}{r}-.338 \\
.002 \\
70\end{array}$ & $\begin{array}{r}.661 \\
.000 \\
70\end{array}$ \\
\hline Age & $\begin{array}{l}\text { Correlation } \\
\text { Significance (1-tailed) } \\
\text { df }\end{array}$ & $\begin{array}{r}.177 \\
.069 \\
70 \\
\end{array}$ & $\begin{array}{r}.130 \\
.139 \\
70 \\
\end{array}$ & $\begin{array}{r}.165 \\
.083 \\
70 \\
\end{array}$ & $\begin{array}{r}-.192 \\
.053 \\
70 \\
\end{array}$ \\
\hline
\end{tabular}




\begin{tabular}{|c|c|c|c|c|c|}
\hline & & RHBCtot & HealthAction & CoaAffect & GH012tot \\
\hline RHBCtot & $\begin{array}{l}\text { Correlation } \\
\text { Significance (1-tailed) } \\
\text { df }\end{array}$ & $\begin{array}{r}1.000 \\
0\end{array}$ & & & \\
\hline HealthAction & $\begin{array}{l}\text { Correlation } \\
\text { Significance (1-tailed) } \\
\text { df }\end{array}$ & $\begin{array}{r}.903 \\
.000 \\
69\end{array}$ & $\begin{array}{r}1.000 \\
0\end{array}$ & & \\
\hline CogAffect & $\begin{array}{l}\text { Correlation } \\
\text { Significance (1-tailed) } \\
\text { df }\end{array}$ & $\begin{array}{r}.612 \\
.000 \\
69\end{array}$ & $\begin{array}{r}.213 \\
.037 \\
69\end{array}$ & $\begin{array}{r}1.000 \\
0\end{array}$ & \\
\hline GHQ12tot & $\begin{array}{l}\text { Correlation } \\
\text { Significance (1-tailed) } \\
\text { df }\end{array}$ & $\begin{array}{r}-.295 \\
.006 \\
69\end{array}$ & $\begin{array}{r}-.196 \\
.050 \\
69\end{array}$ & $\begin{array}{r}-.308 \\
.004 \\
69\end{array}$ & $\begin{array}{r}1.000 \\
0\end{array}$ \\
\hline Ageism & $\begin{array}{l}\text { Correlation } \\
\text { Significance (1-tailed) } \\
\text { df }\end{array}$ & $\begin{array}{r}-.065 \\
.296 \\
69\end{array}$ & $\begin{array}{r}-.121 \\
.156 \\
69\end{array}$ & $\begin{array}{r}.077 \\
.263 \\
69\end{array}$ & $\begin{array}{r}-.115 \\
.169 \\
69\end{array}$ \\
\hline Stigma & $\begin{array}{l}\text { Correlation } \\
\text { Significance (1-tailed) } \\
\text { df }\end{array}$ & $\begin{array}{r}-.076 \\
.265 \\
69\end{array}$ & $\begin{array}{r}-.043 \\
.360 \\
69\end{array}$ & $\begin{array}{r}-.093 \\
.221 \\
69\end{array}$ & $\begin{array}{r}-.107 \\
.188 \\
69\end{array}$ \\
\hline Understan & $\begin{array}{l}\text { Correlation } \\
\text { Significance (1-tailed) } \\
\text { df }\end{array}$ & $\begin{array}{r}.091 \\
.225 \\
69\end{array}$ & $\begin{array}{r}.131 \\
.138 \\
69\end{array}$ & $\begin{array}{r}-.034 \\
.390 \\
69\end{array}$ & $\begin{array}{r}.175 \\
.072 \\
69\end{array}$ \\
\hline AAQtot & $\begin{array}{l}\text { Correlation } \\
\text { Significance (1-tailed) } \\
\text { df }\end{array}$ & $\begin{array}{r}-.370 \\
.001 \\
69\end{array}$ & $\begin{array}{r}-.187 \\
.059 \\
69\end{array}$ & $\begin{array}{r}-.496 \\
.000 \\
69\end{array}$ & $\begin{array}{r}.608 \\
.000 \\
69\end{array}$ \\
\hline Growth & $\begin{array}{l}\text { Correlation } \\
\text { Significance (1-tailed) } \\
\text { df }\end{array}$ & $\begin{array}{r}.397 \\
.000 \\
69\end{array}$ & $\begin{array}{r}.262 \\
.014 \\
69\end{array}$ & $\begin{array}{r}.420 \\
.000 \\
69\end{array}$ & $\begin{array}{r}-.303 \\
.005 \\
69\end{array}$ \\
\hline Loss & $\begin{array}{l}\text { Correlation } \\
\text { Significance (1-tailed) } \\
\text { df }\end{array}$ & $\begin{array}{r}-.119 \\
.162 \\
69\end{array}$ & $\begin{array}{r}.026 \\
.413 \\
69\end{array}$ & $\begin{array}{r}-.319 \\
.003 \\
69\end{array}$ & $\begin{array}{r}.565 \\
.000 \\
69\end{array}$ \\
\hline Physical & $\begin{array}{l}\text { Correlation } \\
\text { Significance (1-tailed) } \\
\text { df }\end{array}$ & $\begin{array}{r}.354 \\
.001 \\
69\end{array}$ & $\begin{array}{r}.231 \\
.026 \\
69\end{array}$ & $\begin{array}{r}.380 \\
.001 \\
69\end{array}$ & $\begin{array}{r}-.450 \\
.000 \\
69\end{array}$ \\
\hline Rametot & $\begin{array}{l}\text { Correlation } \\
\text { Significance (1-tailed) } \\
\text { df }\end{array}$ & $\begin{array}{r}-.288 \\
.007 \\
69\end{array}$ & $\begin{array}{r}-.164 \\
.086 \\
69\end{array}$ & $\begin{array}{r}-.354 \\
.001 \\
69 \\
\end{array}$ & $\begin{array}{r}.687 \\
.000 \\
69\end{array}$ \\
\hline
\end{tabular}


Appendix 4.23: Scatterplots of RHBC, GHQ-12 and Attitudinal Variables

RHBC total score: GHQ-12 Correlation

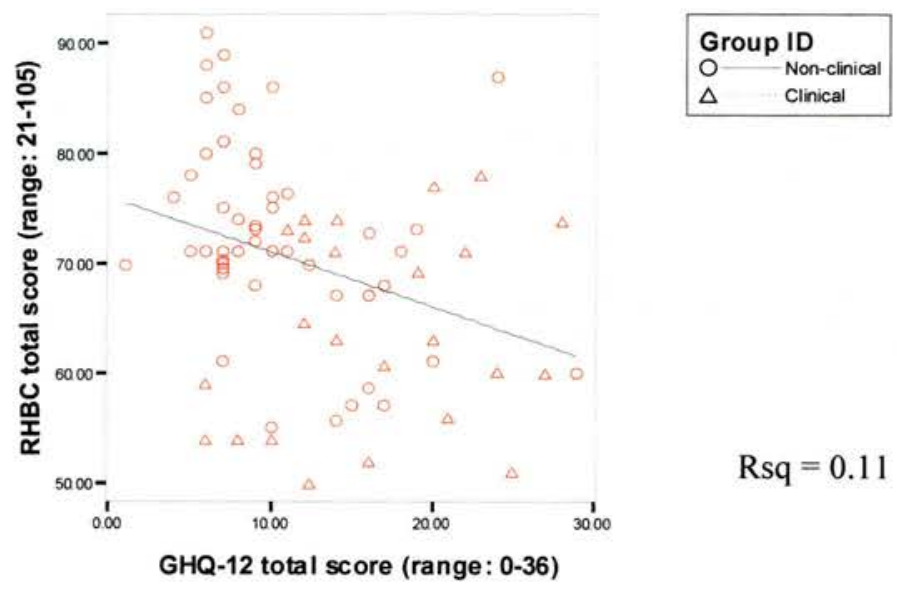

\section{$\underline{\text { RHBC total score : AAQ total score }}$}

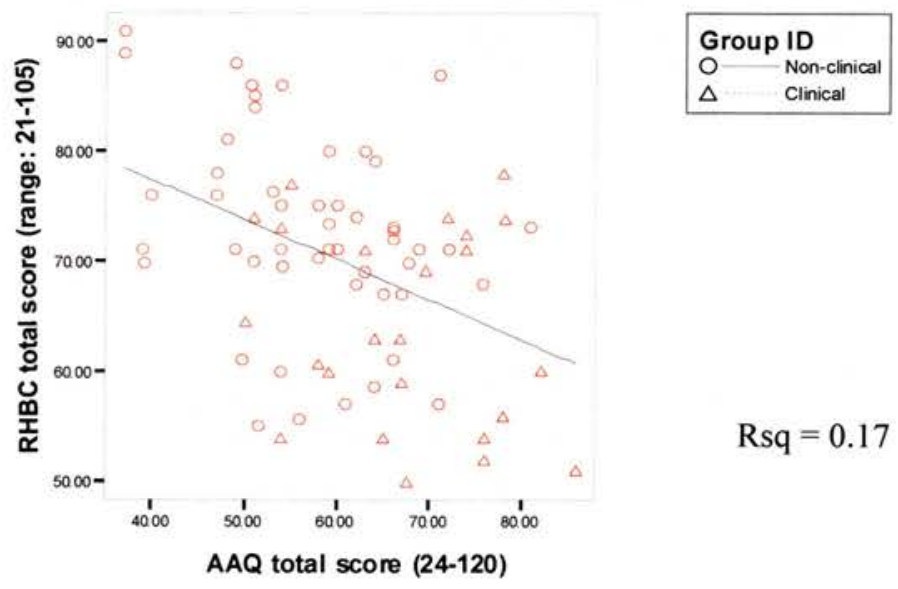

RHBC total score: AAQ 'Physical'

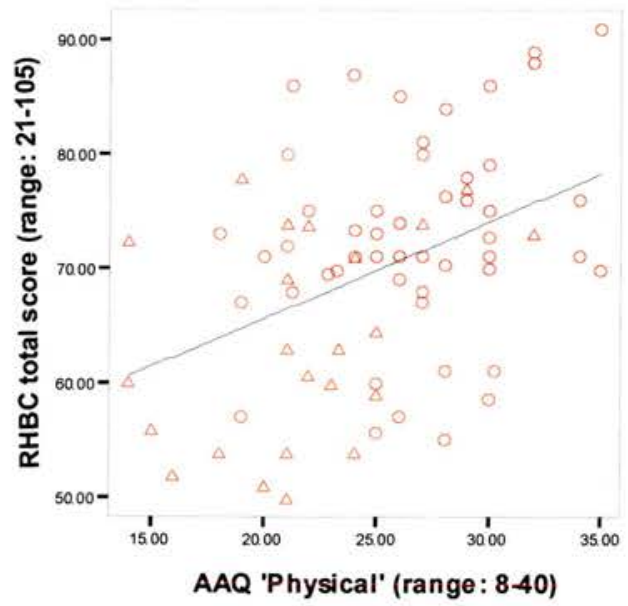

Group ID

O Non-clinical Clinical

Rsq $=0.17$ 
RHBC 'Cognitive Affective' : AAQ total score

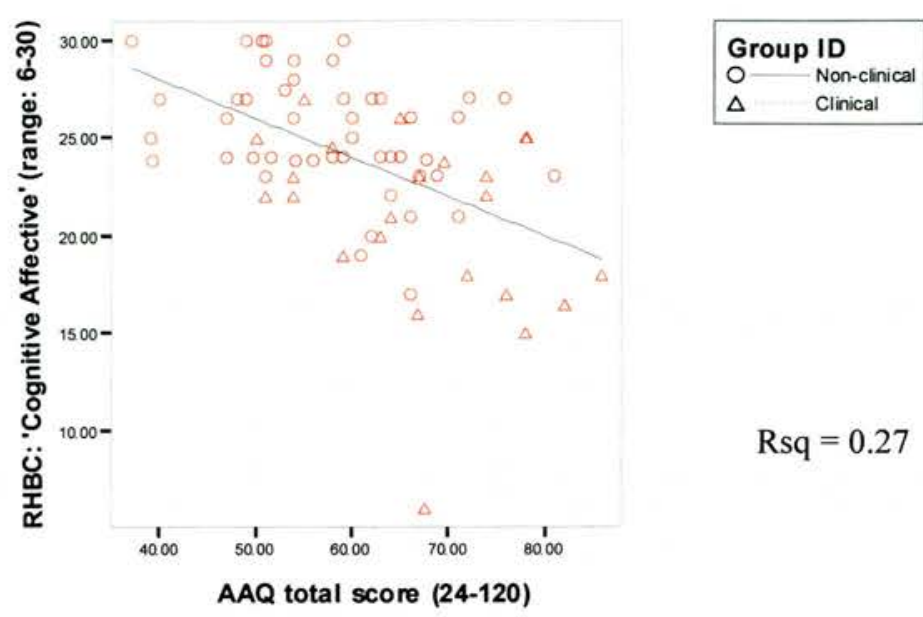

GHQ-12 total score : AAQ total score

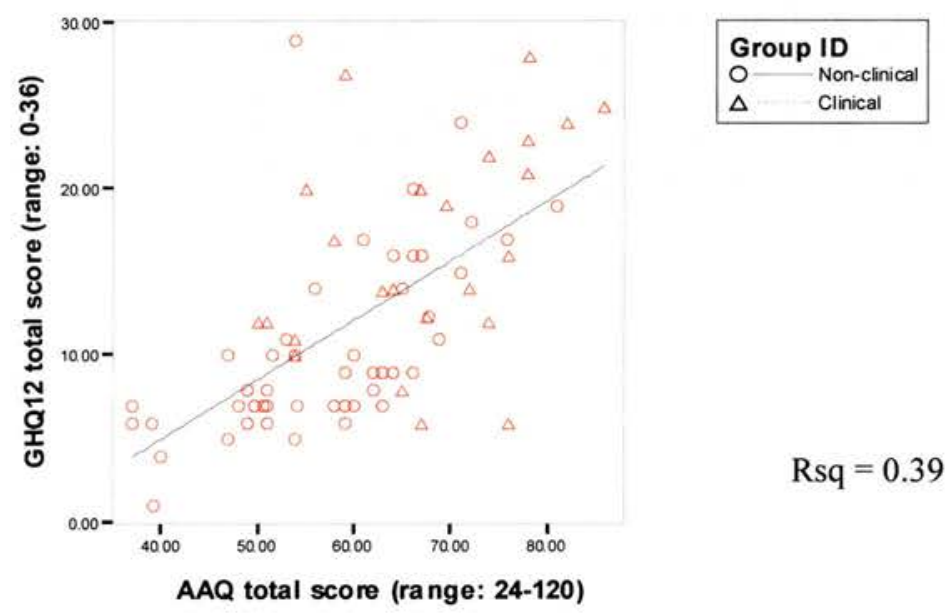

\section{GHQ-12 total score: AAQ 'Physical'}

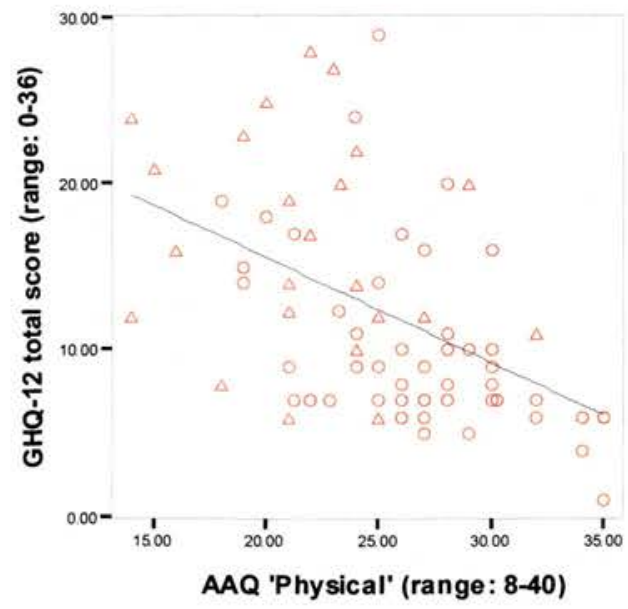

Group ID

O-Non-clinical Clinical

$\mathrm{Rsq}=0.23$ 


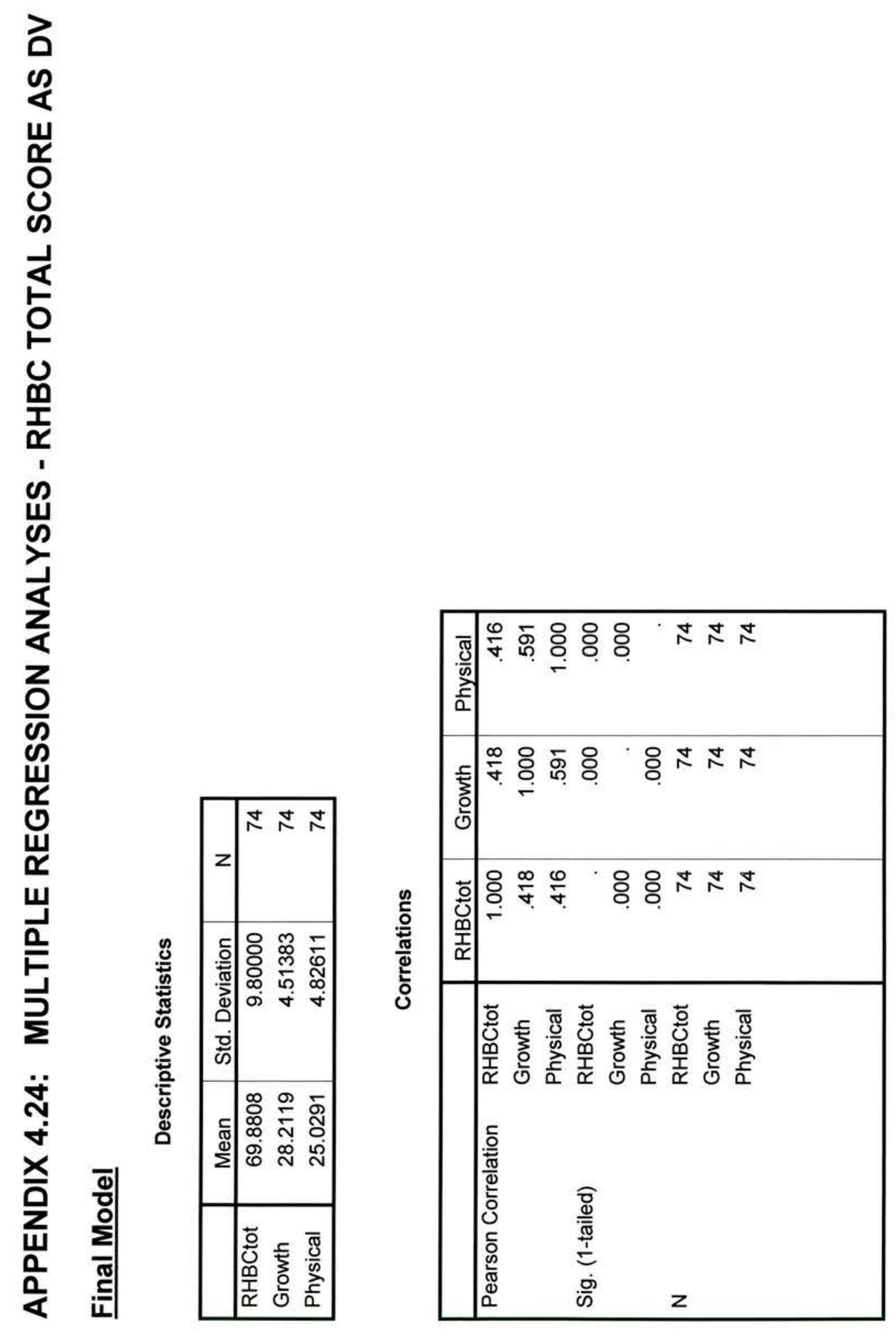



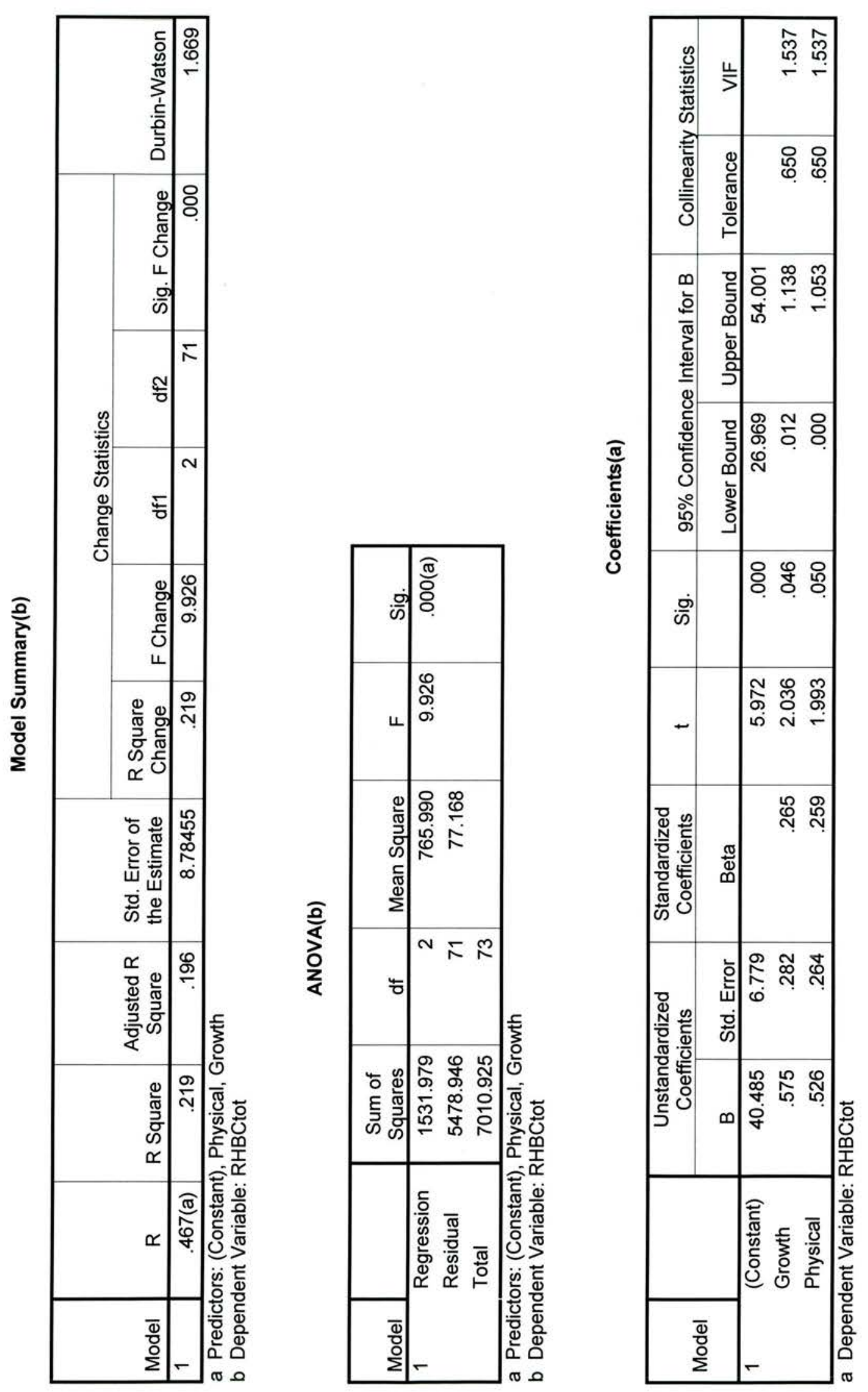

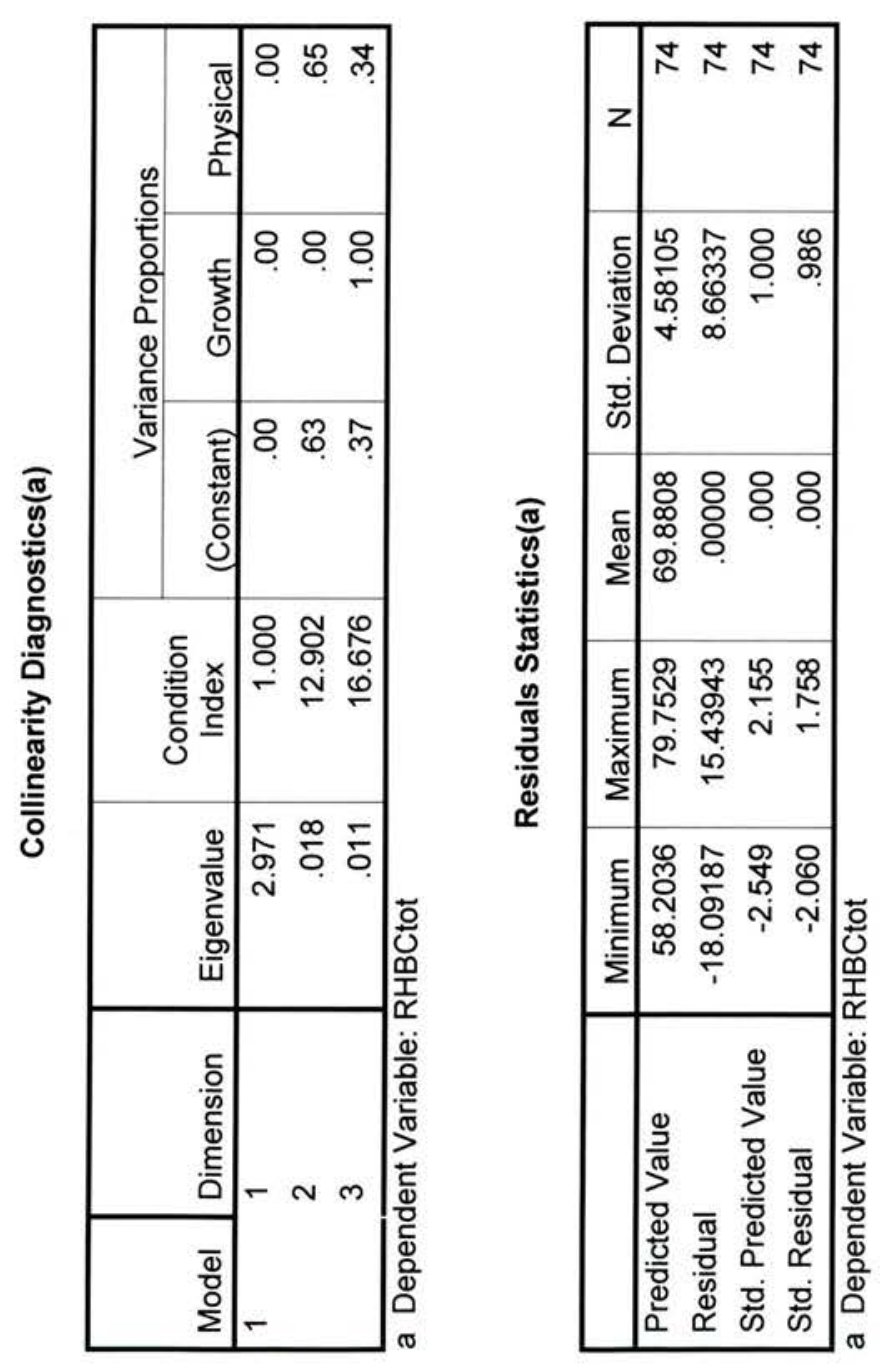


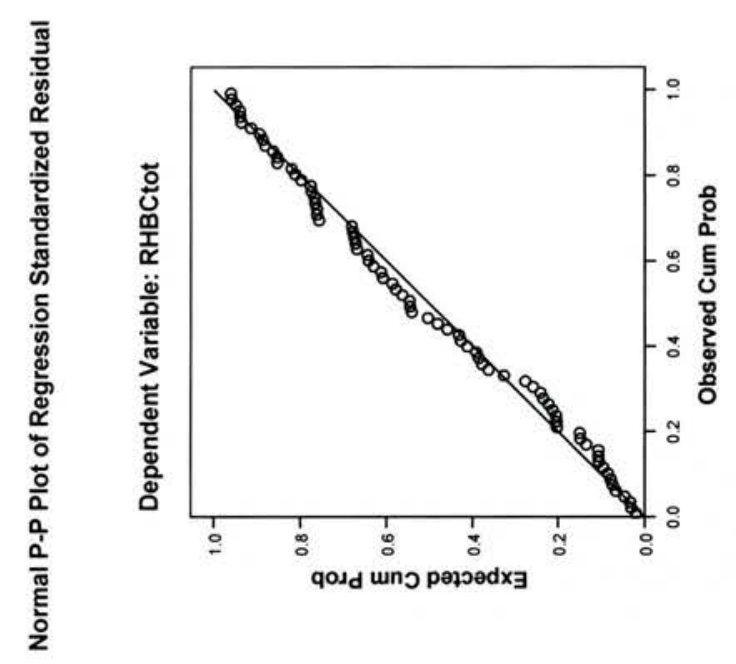

휨

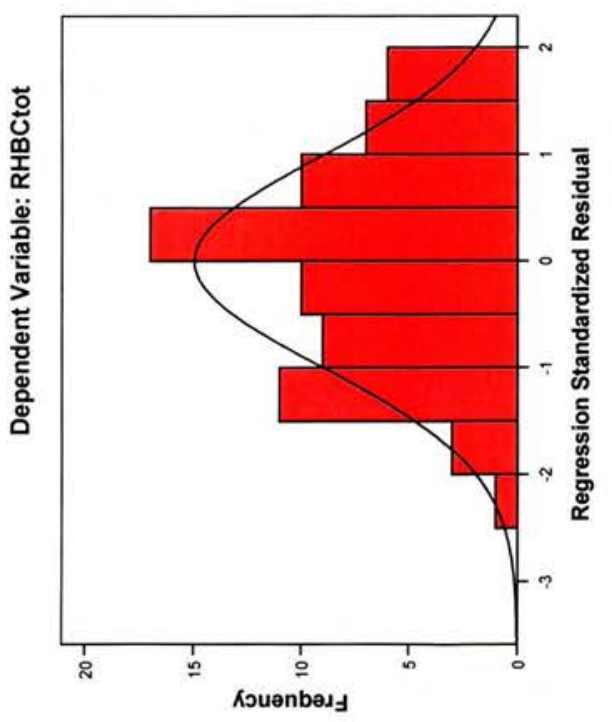




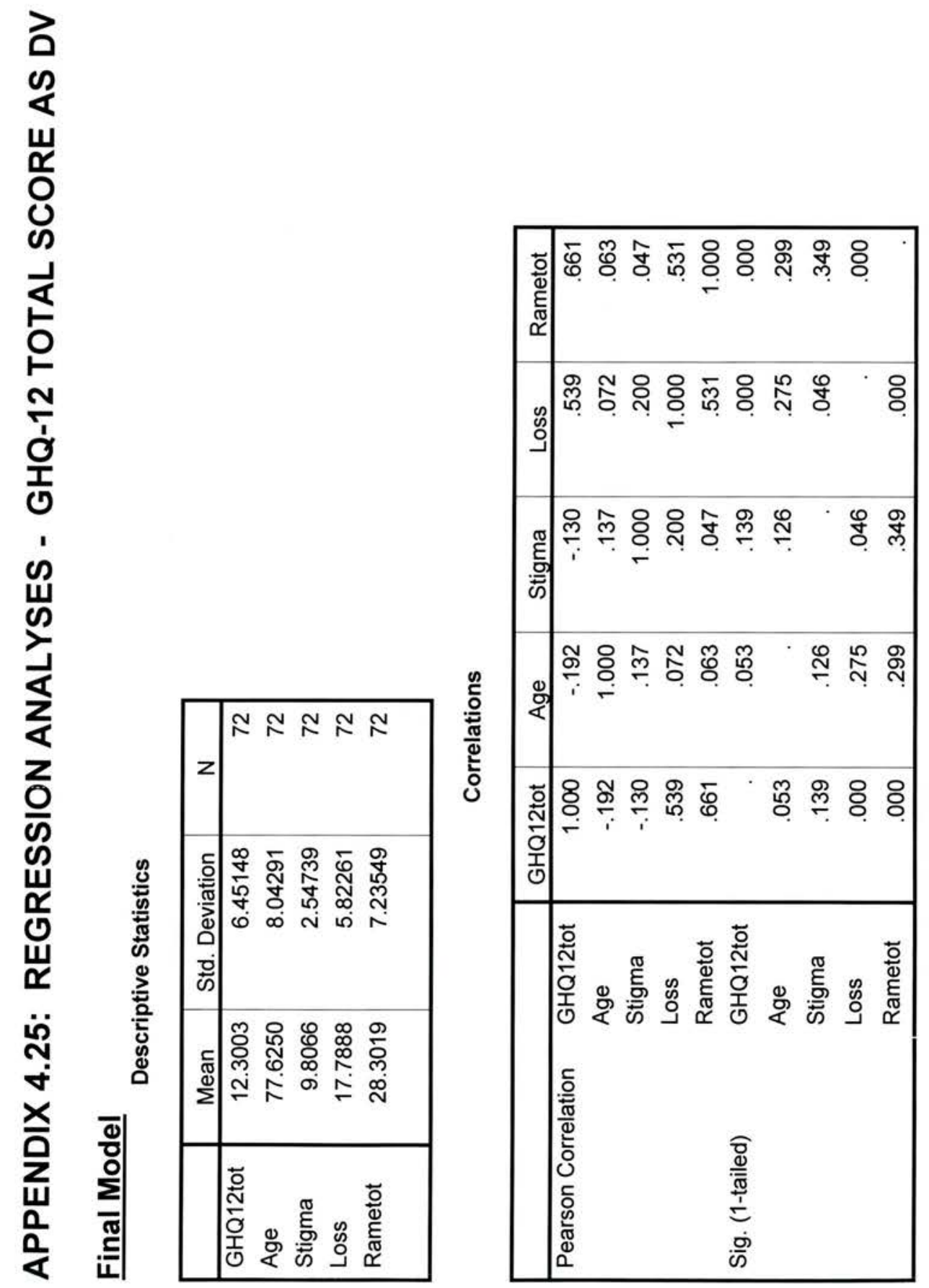



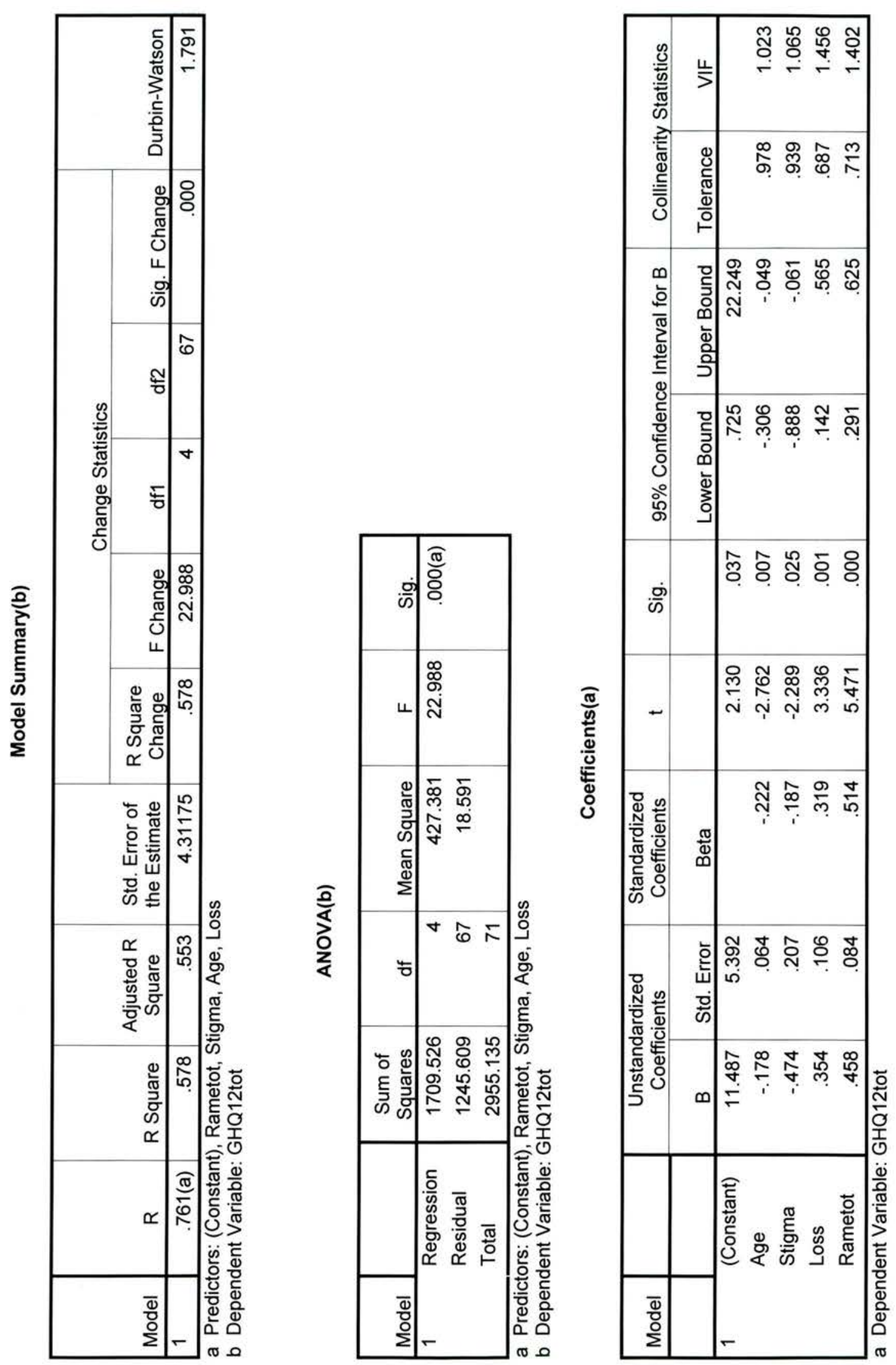


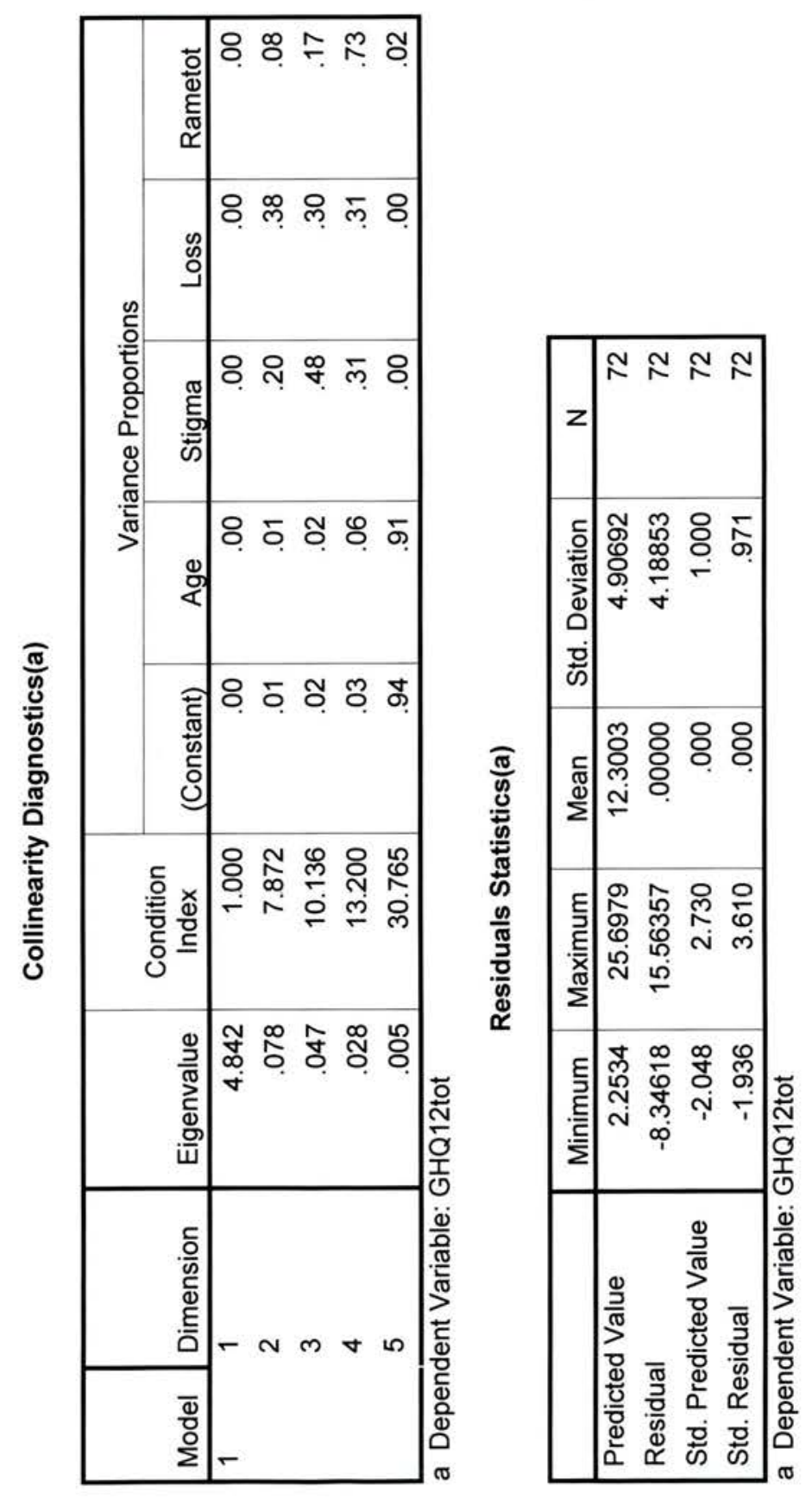



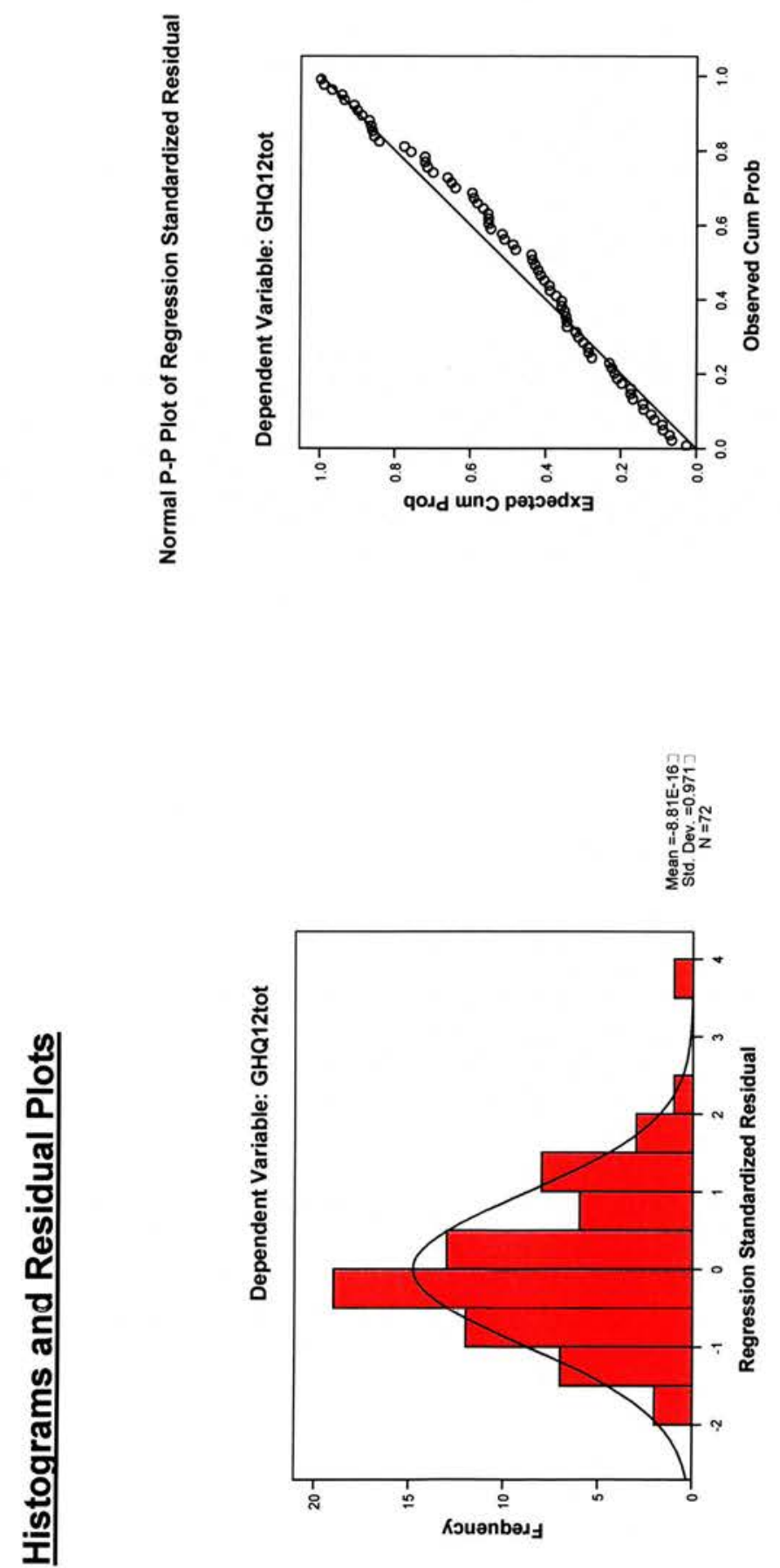